\title{
Guidelines for the Treatment of Hypothyroidism
}

\author{
Prepared by the American Thyroid Association \\ Task Force on Thyroid Hormone Replacement
}

\author{
Jacqueline Jonklaas, ${ }^{1 * \dagger}$ Antonio C. Bianco, ${ }^{2 * *}$ Andrew J. Bauer, ${ }^{3 \dagger}$ Kenneth D. Burman, ${ }^{4 \dagger}$ \\ Anne R. Cappola, ${ }^{5 \dagger}$ Francesco S. Celi, ${ }^{6 *}$ David S. Cooper, ${ }^{7 \dagger}$ Brian W. Kim, ${ }^{2 *}$ Robin P. Peeters, ${ }^{8 *}$ \\ M. Sara Rosenthal, ${ }^{9 \dagger}$ and Anna M. Sawka ${ }^{10 \dagger}$
}

Background: A number of recent advances in our understanding of thyroid physiology may shed light on why some patients feel unwell while taking levothyroxine monotherapy. The purpose of this task force was to review the goals of levothyroxine therapy, the optimal prescription of conventional levothyroxine therapy, the sources of dissatisfaction with levothyroxine therapy, the evidence on treatment alternatives, and the relevant knowledge gaps. We wished to determine whether there are sufficient new data generated by well-designed studies to provide reason to pursue such therapies and change the current standard of care. This document is intended to inform clinical decision-making on thyroid hormone replacement therapy; it is not a replacement for individualized clinical judgment.

Methods: Task force members identified 24 questions relevant to the treatment of hypothyroidism. The clinical literature relating to each question was then reviewed. Clinical reviews were supplemented, when relevant, with related mechanistic and bench research literature reviews, performed by our team of translational scientists. Ethics reviews were provided, when relevant, by a bioethicist. The responses to questions were formatted, when possible, in the form of a formal clinical recommendation statement. When responses were not suitable for a formal clinical recommendation, a summary response statement without a formal clinical recommendation was developed. For clinical recommendations, the supporting evidence was appraised, and the strength of each clinical recommendation was assessed, using the American College of Physicians system. The final document was organized so that each topic is introduced with a question, followed by a formal clinical recommendation. Stakeholder input was received at a national meeting, with some subsequent refinement of the clinical questions addressed in the document. Consensus was achieved for all recommendations by the task force.

Results: We reviewed the following therapeutic categories: (i) levothyroxine therapy, (ii) non-levothyroxinebased thyroid hormone therapies, and (iii) use of thyroid hormone analogs. The second category included thyroid extracts, synthetic combination therapy, triiodothyronine therapy, and compounded thyroid hormones. Conclusions: We concluded that levothyroxine should remain the standard of care for treating hypothyroidism. We found no consistently strong evidence for the superiority of alternative preparations (e.g., levothyroxineliothyronine combination therapy, or thyroid extract therapy, or others) over monotherapy with levothyroxine, in improving health outcomes. Some examples of future research needs include the development of superior biomarkers of euthyroidism to supplement thyrotropin measurements, mechanistic research on serum triiodothyronine

\footnotetext{
${ }^{1}$ Division of Endocrinology, Georgetown University, Washington, DC.

${ }^{2}$ Division of Endocrinology, Rush University Medical Center, Chicago, Illinois.

${ }^{3}$ Division of Endocrinology, Children's Hospital of Philadelphia, Philadelphia, Pennsylvania.

${ }^{4}$ Endocrine Section, Medstar Washington Hospital Center, Washington, DC.

${ }^{5}$ Division of Endocrinology, University of Pennsylvania School of Medicine, Philadelphia, Pennsylvania.

${ }^{6}$ Division of Endocrinology, Virginia Commonwealth University School of Medicine, Richmond, Virginia.

${ }^{7}$ Division of Endocrinology, Johns Hopkins University School of Medicine, Baltimore, Maryland.

${ }^{8}$ Department of Internal Medicine, Erasmus Medical Center, Rotterdam, The Netherlands.

${ }^{9}$ Program for Bioethics, Department of Internal Medicine, University of Kentucky College of Medicine, Lexington, Kentucky.

${ }^{10}$ Division of Endocrinology, University Health Network and University of Toronto, Toronto, Ontario, Canada.

*Task Force Co-chairs.

${ }^{\dagger}$ Clinical Subcommittee.

Translational Science Subcommittee.
} 
levels (including effects of age and disease status, relationship with tissue concentrations, as well as potential therapeutic targeting), and long-term outcome clinical trials testing combination therapy or thyroid extracts (including subgroup effects). Additional research is also needed to develop thyroid hormone analogs with a favorable benefit to risk profile.

\section{INTRODUCTION}

\section{Background, Objectives, and Rationale}

$\mathbf{L}$

EVOTHYROXINE ( $\left.\mathrm{LT}_{4}\right)$ HAS BEEN CONSIDERED the standard of care for treatment of hypothyroidism for many years. This treatment is efficacious when administered orally, has a long serum half-life that permits daily administration, and results in resolution of the signs and symptoms of hypothyroidism in the majority of patients. However, a small proportion of patients being treated for hypothyroidism feel that $\mathrm{LT}_{4}$ therapy is not efficacious in restoring optimum health.

Several recent advances in our understanding of thyroid physiology may shed light on why some patients feel unwell while taking $\mathrm{LT}_{4}$ monotherapy. For example, much has been learned about the sources and regulation of triiodothyronine $\left(\mathrm{T}_{3}\right)$ in the plasma and within specific tissues, as well as about the regulation of thyrotropin (TSH). In addition, new data have emerged on dissatisfaction with $\mathrm{LT}_{4}$ therapy being associated with genetic variation in deiodinases, and fatigue and depression in treated hypothyroid patients being linked with genetic variations in thyroid hormone transporters. The mandate of this task force was to review the goals of $\mathrm{LT}_{4}$ therapy, examine sources of dissatisfaction with $\mathrm{LT}_{4}$ therapy, examine the evidence concerning treatment alternatives, discuss gaps in our current knowledge of these therapies, and determine whether new data provide reason to pursue such therapies.

In this document the latest data regarding combination therapy, liothyronine $\left(\mathrm{LT}_{3}\right)$ monotherapy, compounded thyroid hormones, and nutraceuticals are presented. As a secondary objective, we also review the literature on thyroid hormone analogs. Pharmacology and regulatory aspects of the therapies that we reviewed are discussed. The potential for genetic variations to influence the ability to optimize thyroid hormone therapy is explored. The challenges of titrating thyroid hormone therapy in specific groups such as the pediatric, pregnant, and elderly populations are considered. However, the topic of subclinical hypothyroidism $(\mathrm{SCH})$ is not addressed, other than in the pediatric population, because of prior extensive reviews of this topic in adults (1-4). Thyroid hormone therapy in patients with thyroid cancer is only mentioned if it is germane to the topic being discussed. Our goal is to promote discussion to improve our understanding of these issues, provide recommendations where possible, and to identify areas where further research is needed.

A recent comprehensive document, the "Clinical Practice Guidelines for Hypothyroidism in Adults Co-sponsored by the American Association of Clinical Endocrinologists (AACE) and the American Thyroid Association (ATA)," covers broader aspects of the management of hypothyroidism (3). In addition, two recent consensus documents published by the ATA and the Endocrine Society address the management of hypothyroidism during pregnancy $(1,2)$. While this document was in preparation, guidelines from the European Thyroid Association (ETA) specifically addressing the issue of combined treatment with $\mathrm{LT}_{4}$ and $\mathrm{LT}_{3}$ were published (5). We acknowledge these guidelines, and our document was prepared with a goal of minimizing redundancy. We intended that the features that would distinguish this document from these guidelines would be (i) attention to the basic science and translational underpinning for the various thyroid hormone therapies, (ii) extensive consideration of ethical issues, (iii) focus on treatment itself, as opposed to other aspects of diagnosis and management, and most importantly (iv) focus on evaluation of treatment alternatives. We also wished to explore promising preclinical data for potential future therapies. For each topic, we evaluated the scientific validity of the studies cited.

The target audience for these guidelines includes clinicians providing care to patients with hypothyroidism. We outline what we believe is rational and optimal medical practice based on our evaluation of the evidence at the time of publication. However, areas of uncertainty and difference of opinion among experts remain, and it is not the intent of these guidelines to replace clinical judgment or individual decisionmaking. Rather, these recommendations are intended to inform the clinical decision-making process.

\section{Approach Utilized in Conducting This Review}

Our task force was commissioned and approved by officers of the ATA. We formed in the summer of 2011 and our composition included members with particular expertise in mechanistic and translational science (four members), members with particular expertise in clinical thyroidology (six members), a member with a background in pediatric endocrinology, a member with expertise in design and evaluation of health research, and a bioethicist. As of October 2011, we were redirected and specifically asked to develop clinical practice guidelines. Our task force met face to face in October 2011, June 2012, September 2012, June 2013, October 2013, and June 2014 with seven interim discussions using conference calls. A spring meeting of the ATA that was open to all potential stakeholders (e.g., health care providers, patients, public) was held on April 25 and 26, 2013, to highlight and promote broad discussion regarding relevant and topical issues (6). Information needs identified by stakeholders at this meeting were incorporated in task force group decisions in considering topics for review.

Task force members identified 24 questions relevant to the treatment of hypothyroidism, which were divided among the members. The clinical literature relating to each question was then reviewed by a primary reviewer, who summarized the findings, and compiled a response to the question in the form of a recommendation. The summary of the literature and response to each question were next revised by a secondary reviewer. Clinical reviews were supplemented, when relevant, with related mechanistic and bench research literature reviews, performed by a translational scientist and reviewed by a panel 
subgroup of basic scientists. These sections served as the mechanistic background for the clinical reviews. Task force members also reviewed published guidelines from other groups (and related cross-references) when available. For some topics, such as compounding of thyroid hormones, nutraceuticals, and Wilson's syndrome, when sparse published medical literature was available, we consulted the Internet using Google search. Ethics reviews were provided, when relevant, by a bioethicist, with a second review by one or more task force members with clinical expertise.

The development of each recommendation was as follows: the first reviewer developed the recommendation, reached consensus with the second reviewer, then presented to the group for input, with revisions until the entire group reached consensus. Opportunity for revision existed at all steps of the review process. Formal consensus was obtained among group members for all recommendations. There was also agreement regarding the interpretation of areas of uncertainty discussed in the text, except in two instances in which the differences in interpretation are noted. Each recommendation along with the associated literature review was assembled into a document. The responses to questions were formatted, when possible, in the form of a formal clinical recommendation statement. However, given the broad nature of questions posed by the task force and the broad nature of reviewed literature (including basic science and ethics literature), not all responses were amenable to a formal clinical recommendation, and in such circumstances, a summary response statement without a formal clinical recommendation was developed. For clinical recommendations, the supporting evidence was appraised, and the strength of the clinical recommendations was described, using the American College of Physicians (ACP) system (7). The quality of basic science and ethics articles was not appraised, due to lack of availability of appropriate grading systems for this purpose. The final document is organized so that each topic is introduced with a question, which is followed by a formal clinical recommendation, and completed with a literature review. When a formal clinical recommendation was not feasible because of sparse evidence, or because the question did not lend itself to a recommendation, an ungraded summary statement without a formal recommendation is provided.

In searching for relevant citations for review, task force members conducted electronic database searches of PubMed, using relevant search terms. Databases searches were generally updated at least once. A language restriction was not imposed on studies considered for review. Only published studies were eligible for inclusion for review for the purpose of formulating clinical recommendations. Electronic searches were supplemented with hand searches, including cross-referencing of included articles and reviews, as well as articles suggested by other task force members. For the clinical topic reviews, the authors preferentially selected articles of the highest methodologic quality for inclusion (e.g., randomized controlled trials and meta-analyses/systematic reviews of randomized controlled trials), but when these were not available, articles with lower quality of evidence were reviewed. Greater credence was afforded to such articles when the findings were consistent or congruent.

Clinical recommendations were graded according to the ACP grading system for evidence and recommendations (7) (see Table 1). Using this system as a foundation, the strength of each clinical recommendation was reported according to the
Table 1. The American College of Physicians' Guideline Grading System

\begin{tabular}{|c|c|c|}
\hline \multirow[b]{2}{*}{$\begin{array}{l}\text { Quality of } \\
\text { evidence }\end{array}$} & \multicolumn{2}{|c|}{ Strength of recommendation } \\
\hline & $\begin{array}{l}\text { Benefits clearly } \\
\text { outweigh risks and } \\
\text { burden or risks } \\
\text { and burden clearly } \\
\text { outweigh benefits }\end{array}$ & $\begin{array}{l}\text { Benefits finely } \\
\text { balanced with } \\
\text { risks and } \\
\text { burden }\end{array}$ \\
\hline High & Strong & Weak \\
\hline Moderate & Strong & Weak \\
\hline Low & Strong & Weak \\
\hline
\end{tabular}

Reprinted, with permission, from The Development of Clinical Practice Guidelines and Guidance Statements of the American College of Physicians. Ann Intern Med 2010;153:194-199.

following categories: Strong, Weak, or No Recommendation due to Insufficient Evidence. The quality of evidence upon which the recommendation was based was graded as strong, moderate, low, or insufficient. The interpretation of this grading system is explained in detail in Table 2, which is a reproduction of Table 2 from the original article (7). It should be noted that in this grading system strong recommendations can be made based on low-quality evidence if it is nevertheless assessed that benefits clearly outweigh the risks and burden. Such strong recommendations may change when higher-quality evidence becomes available.

Our final guidelines document was approved by the board of directors of the ATA, underwent a 1-month period of open comment by the ATA membership, and was peer-reviewed by reviewers for the journal Thyroid. Revisions were made in response to each of these reviews. As with other ATAsponsored guidelines, we anticipate that this document will be revised and updated on a 5-year cycle.

\section{Management of Potential Competing Interests}

Special attention was paid to potential conflicts of interest. Possible conflicts of interest of potential task force members were reviewed by the officers of the ATA. No confirmed task force member was considered to have a serious conflict that precluded inclusion on the task force. As per ATA policy, potential competing interests of task force members were recorded at baseline and updated on a yearly basis. The competing interests of members of this task force were shared with the entire group and are listed separately. The task force members volunteered their time and expertise and did not receive funds from the ATA. No funds were received from commercial sources for the development of this document. Task force members did not receive any funding or gifts for their participation and paid for their own travel expenses and registration related to face-to-face meetings. Task force members were encouraged to share any questions or concerns about potential competing interests (or the appearance of any competing interests) with the co-chairs of the task force, and, where appropriate, any such questions or concerns were formally reviewed by the ATA Ethics committee. As per ATA policy, the results of any reviews by the Ethics Committee were shared with the entire group, with an opportunity to voice any additional potential questions or concerns relating to the review. 


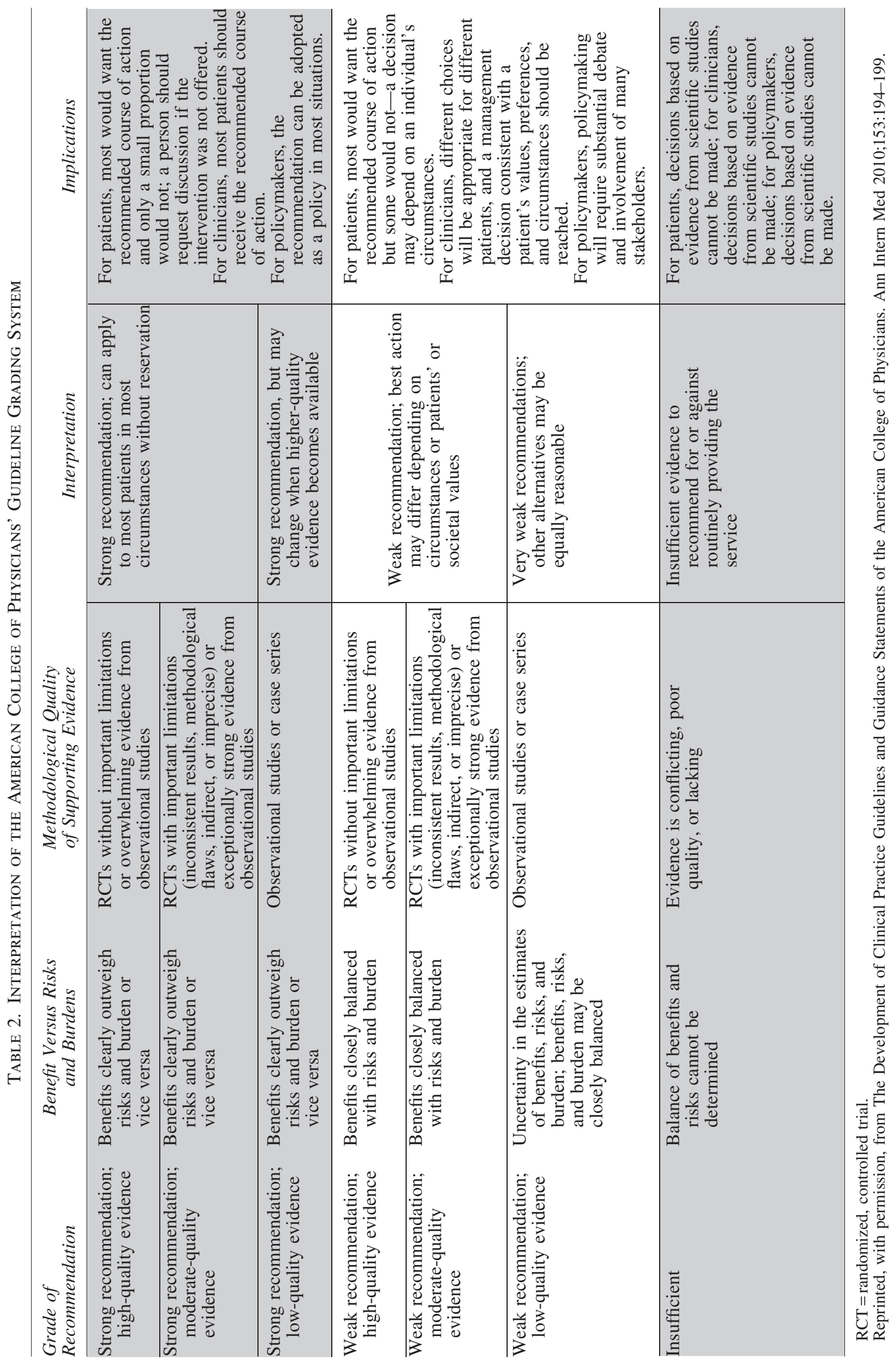


ORGANIZATION OF GUIDELINES:

SECTION I. LEVOTHYROXINE THERAPY

\begin{tabular}{llr}
\hline Number & \multicolumn{1}{c}{ Question } & Page \\
\hline & & Levothyroxine Therapy and Endpoints During Therapy \\
1a & Is levothyroxine monotherapy considered to be the standard of care for hypothyroidism? & 1676 \\
lb & What are the clinical and biochemical goals for levothyroxine replacement in primary & 1677 \\
& hypothyroidism? & 1678
\end{tabular}

assessing adequacy of levothyroxine replacement in primary hypothyroidism?

1d Are tissue markers of thyroid hormone action helpful in determining the adequacy of levothyroxine replacement in primary hypothyroidism?

2a Is there a clinical rationale for prescribing brand-name levothyroxine preparations in 1679 preference to generic levothyroxine?

$2 \mathrm{~b} \quad$ Are there situations in which therapy with levothyroxine dissolved in glycerin and supplied

1681 in gelatin capsules may have advantages over standard levothyroxine?

Levothyroxine Absorption and Metabolism
Haw should levothyroxine administration be timed with respect to meals and beverages
in order to maintain maximum, consistent absorption?

$3 \mathrm{~b} \quad$ Are there medications and supplements that should not be co-administered with levothyroxine

1682 in order to avoid impaired absorption?

$3 \mathrm{c} \quad$ Are there gastrointestinal conditions that should be considered when a patient's levothyroxine

1683 dose is much higher than expected?

3d Are different levothyroxine products associated with different absorption, such that a switch

1684

to a different brand name or generic is associated with a significantly different serum thyrotropin?

3e What medications may alter a patient's levothyroxine requirement by affecting either metabolism or binding to transport proteins?

\begin{tabular}{|c|c|}
\hline & Levothyroxine Dosage \\
\hline $4 \mathrm{a}$ & $\begin{array}{l}\text { What factors determine the levothyroxine dose required by a hypothyroid patient for reaching } \\
\text { the appropriate serum thyrotropin goal? }\end{array}$ \\
\hline $4 b$ & What is the best approach to initiating and adjusting levothyroxine therapy? \\
\hline $4 \mathrm{c}$ & What are the potential deleterious effects of excessive levothyroxine? \\
\hline \multirow[t]{2}{*}{$4 d$} & What are the potential deleterious effects of inadequate levothyroxine? \\
\hline & Levothyroxine and Other Nonhypothyroid Medical Conditions \\
\hline $5 \mathrm{a}$ & $\begin{array}{l}\text { What is the appropriate management of perceived allergy to the constituents of levothyroxine } \\
\text { or intolerance to levothyroxine? }\end{array}$ \\
\hline $5 b$ & $\begin{array}{l}\text { How do co-existent medical conditions (e.g., atherosclerotic coronary artery disease) affect } \\
\text { the management of levothyroxine replacement therapy? }\end{array}$ \\
\hline \multirow[t]{2}{*}{$5 c$} & $\begin{array}{l}\text { How do coexistent psychosocial, behavioral, and mental health conditions (such as addiction, } \\
\text { somatization disorder, and depression) affect the management of levothyroxine therapy? }\end{array}$ \\
\hline & Levothyroxine Therapy in Specific Subpopulations \\
\hline $6 \mathrm{a}$ & How should levothyroxine therapy be managed in the elderly with hypothyroidism? \\
\hline $6 \mathrm{~b}$ & How should levothyroxine therapy be managed in pregnant women with hypothyroidism? \\
\hline $6 \mathrm{c}$ & $\begin{array}{l}\text { How should levothyroxine therapy be managed in infants and children with overt } \\
\text { hypothyroidism? }\end{array}$ \\
\hline $6 \mathrm{~d}$ & How should levothyroxine therapy be managed in children with subclinical hypothyroidism? \\
\hline \multirow[t]{2}{*}{$6 e$} & $\begin{array}{l}\text { How should levothyroxine therapy be managed in individuals who have elevated thyrotropin } \\
\text { values due to nonadherence? }\end{array}$ \\
\hline & Levothyroxine and Serum Triiodothyronine Concentrations \\
\hline $7 a$ & $\begin{array}{l}\text { Are variations in serum triiodothyronine concentrations within the reference range of } \\
\text { physiologic or clinical significance? In addition, are mildly low serum triiodothyronine } \\
\text { concentrations of clinical significance? }\end{array}$ \\
\hline $7 b$ & $\begin{array}{l}\text { Does levothyroxine therapy that returns the serum thyrotropin levels of hypothyroid patients } \\
\text { to the reference range also result in normalization of their serum triiodothyronine levels? }\end{array}$ \\
\hline $7 \mathrm{c}$ & $\begin{array}{l}\text { Is there evidence of discordance between the thyroid hormone status of different tissues and } \\
\text { the serum thyrotropin concentration? }\end{array}$ \\
\hline $7 d$ & $\begin{array}{l}\text { Should levothyroxine therapy for hypothyroidism, particularly in specific subgroups such as } \\
\text { those with obesity, depression, dyslipidemia, or who are athyreotic, be targeted to achieve } \\
\text { high-normal serum triiodothyronine levels or low-normal serum thyrotropin levels? }\end{array}$ \\
\hline
\end{tabular}
Levothyroxine Dosage

hat factors determine the levothyroxine dose required by a hypothyroid patient for reaching

1686

the appropriate serum thyrotropin goal?

What is the best approach to initiating and adjusting levothyroxine therapy? 1687

What are the potential deleterious effects of excessive levothyroxine?

\section{Levothyroxine and Other Nonhypothyroid Medical Conditions}

1688 or intolerance to levothyroxine?

How do co-existent medical conditions (e.g., atherosclerotic coronary artery disease) affect

1689

1689 somatization disorder, and depression) affect the management of levothyroxine therapy?

\section{Levothyroxine Therapy in Specific Subpopulations}

How should levothyroxine therapy be managed in the elderly with hypothyroidism?

ow should levothyroxine therapy be managed in pregnant women with hypothyroidism? hypothyroidism?

How should levothyroxine therapy be managed in children with subclinical hypothyroidism? 1691 w should levothyroxine therapy be managed in individuals who have elevated thyrotropin 1693

\section{Levothyroxine and Serum Triiodothyronine Concentrations} physiologic or clinical significance? In addition, are mildly low serum triiodothyronine

to the reference range also result in normalization of their serum triiodothyronine levels? the serum thyrotropin concentration? those with obesity, depression, dyslipidemia, or who are athyreotic, be targeted to achieve high-normal serum triiodothyronine levels or low-normal serum thyrotropin levels? 
SECTION I. LEVOTHYROXINE THERAPY (CONTINUED)

\begin{tabular}{|c|c|c|}
\hline Number & Question & Page \\
\hline \multicolumn{3}{|c|}{ Levothyroxine Therapy in Secondary Hypothyroidism } \\
\hline $8 \mathrm{a}$ & $\begin{array}{l}\text { What biochemical goals should be employed for levothyroxine replacement in patients with } \\
\text { secondary hypothyroidism? }\end{array}$ & 1698 \\
\hline $8 \mathrm{~b}$ & $\begin{array}{l}\text { Are clinical parameters useful for assessing adequacy of levothyroxine replacement } \\
\text { in patients with secondary hypothyroidism? }\end{array}$ & 1699 \\
\hline $8 \mathrm{c}$ & $\begin{array}{l}\text { Are tissue markers of thyroid hormone action helpful for management of levothyroxine } \\
\text { replacement in patients with secondary hypothyroidism? }\end{array}$ & 1699 \\
\hline \multicolumn{3}{|c|}{ Patient Satisfaction with Levothyroxine Therapy } \\
\hline $9 \mathrm{a}$ & $\begin{array}{l}\text { What tools may be useful in the clinical or research setting, to measure the impact of } \\
\text { levothyroxine replacement for primary hypothyroidism on patients' physical or psychological } \\
\text { well-being, treatment satisfaction, or treatment preferences? }\end{array}$ & 1700 \\
\hline $9 b$ & $\begin{array}{l}\text { What approach should be taken in patients treated for hypothyroidism who have normal } \\
\text { serum thyrotropin values but still have unresolved symptoms? }\end{array}$ & 1700 \\
\hline \multicolumn{3}{|c|}{ Use of Levothyroxine in Euthyroid Individuals } \\
\hline $10 \mathrm{a}$ & $\begin{array}{l}\text { Is there a role for the use of levothyroxine to treat biochemically euthyroid patients with } \\
\text { symptoms that overlap with those of hypothyroidism? }\end{array}$ & 1701 \\
\hline $10 \mathrm{~b}$ & Is there a role for the use of levothyroxine to treat euthyroid patients with depression? & 1702 \\
\hline $10 \mathrm{c}$ & Is there a role for the use of levothyroxine to treat euthyroid patients with obesity? & 1702 \\
\hline $10 \mathrm{~d}$ & Is there a role for the use of levothyroxine to treat euthyroid patients with urticaria? & 1703 \\
\hline $10 \mathrm{e}$ & What is the recommended approach to treating factitious thyrotoxicosis? & 1703 \\
\hline 11 & $\begin{array}{l}\text { Clinical Ethics Considerations in Levothyroxine Treatment } \\
\text { What are the ethical obligations of clinicians in treating hypothyroidism? }\end{array}$ & \\
\hline
\end{tabular}

SECTION II. THERAPIES OTHER THAN LEVOTHYROXINE ALONE

Thyroid Extracts

12 In adults requiring thyroid hormone replacement treatment for primary hypothyroidism, is treatment with thyroid extracts superior to treatment with levothyroxine alone? Synthetic Combination Therapy and the Rationale for Its Use

13a Do genetic variants in thyroid hormone pathway genes (deiodinases or thyroid hormone transporters) affect the serum or tissue levels of thyroid hormones in healthy euthyroid individuals or hypothyroid patients taking replacement therapy?

13b In adults requiring thyroid hormone replacement treatment for primary hypothyroidism, is combination treatment including levothyroxine and liothyronine superior to the use of levothyroxine alone?

13c In adults requiring thyroid hormone replacement treatment for primary hypothyroidism who feel unwell while taking levothyroxine, is combination treatment including levothyroxine and liothyronine superior to the use of levothyroxine alone?

13d Should genetic characterization according to type 2 deiodinase gene polymorphism status be used to guide the use of combination synthetic levothyroxine and liothyronine therapy in hypothyroidism, in order to optimize biochemical and clinical outcomes?

Liothyronine Monotherapy for Hypothyroidism

14 Are there data regarding therapy with triiodothyronine alone, either as standard liothyronine or as sustained release triiodothyronine, that support the use of triiodothyronine therapy alone for the treatment of hypothyroidism?

\section{Liothyronine Monotherapy in Euthyroid Patients}

$15 \mathrm{a}$ Is there a role for the use of liothyronine to treat biochemically euthyroid patients with depression? 1714

$15 \mathrm{~b} \quad$ Is there a role for the use of liothyronine to treat biochemically euthyroid patients with obesity? Compounded Thyroid Hormones

16 What is the recommendation regarding therapy with compounded thyroid hormones (either levothyroxine or liothyronine) for treatment of hypothyroidism based on current evidence? Nutraceuticals

17 Is there a role for the use of dietary supplementation, nutraceuticals, and over-the-counter products 1716 in either hypothyroid or euthyroid individuals? Ethical Considerations in Hypothyroidism Research

18 What are the research ethics issues involved in evaluating or designing clinical trials for the treatment 1718 of hypothyroidism?

Wilson's Syndrome

19 Is there evidence for the existence of "Wilson's temperature syndrome" and a rationale for use of escalating doses of triiodothyronine? 
SECTION III. HOSPITALIZED PATIENTS

\begin{tabular}{|c|c|c|}
\hline Number & Question & Page \\
\hline \multicolumn{3}{|c|}{ Levothyroxine in Hospitalized Patients } \\
\hline $20 \mathrm{a}$ & $\begin{array}{l}\text { In hospitalized but not critically ill patients with known pre-existing hypothyroidism, should } \\
\text { levothyroxine therapy be re-evaluated based on an elevated serum thyrotropin measurement? }\end{array}$ & 1720 \\
\hline $20 \mathrm{~b}$ & $\begin{array}{l}\text { In hospitalized but not critically ill patients in whom levothyroxine replacement is instituted or } \\
\text { increased, should the therapeutic goal be normalization of serum thyrotropin? }\end{array}$ & 1720 \\
\hline $20 \mathrm{c}$ & $\begin{array}{l}\text { In hospitalized but not critically ill patients treated with levothyroxine replacement what } \\
\text { formulation and route of administration are recommended? }\end{array}$ & 1720 \\
\hline $20 d$ & $\begin{array}{l}\text { In hospitalized but not critically ill patients about to be treated with levothyroxine should the } \\
\text { possibility of adrenal insufficiency be excluded? }\end{array}$ & 1720 \\
\hline \multicolumn{3}{|c|}{ Myxedema Coma } \\
\hline $21 \mathrm{a}$ & $\begin{array}{l}\text { In patients with myxedema coma being treated with levothyroxine, what route of administration } \\
\text { should be used? }\end{array}$ & 1721 \\
\hline $21 b$ & $\begin{array}{l}\text { In patients with myxedema coma being treated with levothyroxine, should empiric glucocorticoid } \\
\text { coverage be provided? }\end{array}$ & 1721 \\
\hline $21 \mathrm{c}$ & $\begin{array}{l}\text { In patients with myxedema coma being treated with levothyroxine, should liothyronine therapy } \\
\text { also be initiated? }\end{array}$ & 1721 \\
\hline $21 d$ & $\begin{array}{l}\text { In patients with myxedema coma being treated with levothyroxine, what therapeutic endpoints } \\
\text { should be targeted? }\end{array}$ & 1721 \\
\hline \multicolumn{3}{|c|}{ Low Triiodothyronine Concentrations in Hospitalized Patients } \\
\hline $22 \mathrm{a}$ & $\begin{array}{l}\text { In hospitalized adult patients exhibiting the "nonthyroidal illness syndrome," should thyroid } \\
\text { hormone replacement be instituted with levothyroxine? }\end{array}$ & 1722 \\
\hline $22 \mathrm{~b}$ & $\begin{array}{l}\text { In hospitalized adult patients exhibiting the "nonthyroidal illness syndrome," should thyroid } \\
\text { hormone replacement be instituted with liothyronine? }\end{array}$ & 1722 \\
\hline $22 \mathrm{c}$ & $\begin{array}{l}\text { In hospitalized adult patients with cardiac dysfunction, such as advanced heart failure, and low } \\
\text { serum triiodothyronine concentrations, should thyroid hormone replacement be instituted } \\
\text { with liothyronine? }\end{array}$ & 1724 \\
\hline \multicolumn{3}{|c|}{ SECTION IV. USE OF THYROID HORMONE ANALOGS } \\
\hline \multicolumn{3}{|c|}{ Thyroid Hormone Analogs and Euthyroid Patients } \\
\hline 23 & $\begin{array}{l}\text { Should thyroid hormone analog therapy be used in euthyroid individuals with non-hypothyroid- } \\
\text { related medical conditions (such as dyslipidemia) based on current evidence? }\end{array}$ & 1725 \\
\hline \multicolumn{3}{|c|}{ Thyroid Hormone Analogs and Thyroid Hormone Resistance } \\
\hline 24a & $\begin{array}{l}\text { What are the clinical and biochemical goals for treatment of patients who have genetic } \\
\text { syndromes of resistance to thyroid hormone? }\end{array}$ & 1726 \\
\hline $24 \mathrm{~b}$ & $\begin{array}{l}\text { What is the role of thyroid hormone analogs in treating patients who have genetic syndromes } \\
\text { of resistance to thyroid hormone? }\end{array}$ & 1726 \\
\hline
\end{tabular}

\section{SECTION I. LEVOTHYROXINE THERAPY}

\section{Levothyroxine Therapy and Endpoints During Therapy}

\section{1a. Is levothyroxine monotherapy considered to be the standard of care for hypothyroidism?}

\section{- RECOMMENDATION}

Levothyroxine is recommended as the preparation of choice for the treatment of hypothyroidism due to its efficacy in resolving the symptoms of hypothyroidism, long-term experience of its benefits, favorable side effect profile, ease of administration, good intestinal absorption, long serum halflife, and low cost.

\section{Strong recommendation. Moderate quality evidence.}

\section{Mechanistic background}

Thyroid hormone action is an important determinant of development and growth, and in adults plays a critical role in the regulation of the function and metabolism of virtually every organ system (8,9). Tissue-specific modulation of the thyroid hormone action is achieved by a complex and redundant control system that includes thyroid hormone secretion, plasma transport, transmembrane transport, activation/inactivation, and interaction with nuclear receptor isoforms and their co-regulators.

Hypothyroid patients are deficient in endogenously produced thyroid hormone. The rationale for the therapeutic use of $L T_{4}$ in the treatment of hypothyroidism lies in the peripheral conversion of the exogenously administered pro-hormone thyroxine $\left(T_{4}\right)$ into its active metabolite $T_{3}(10)$. This activating conversion is accomplished by two enzymes, the type 1 (D1) and type 2 (D2) deiodinases. A third deiodinase, type 3 deiodinase (D3) participates in the clearance of both serum $T_{4}$ and $T_{3}$ (11). Understanding the impact of the deiodinases on thyroid hormone action requires knowledge of their kinetics, tissue distribution, subcellular localization, and regulation (see Table 3). Currently, no clinical assays measure the deiodinase activities directly, thus their contributions to thyroid hormone homeostasis in humans have been established in the research setting in which thyroid hormone levels, kinetic studies with radioactive iodothyronines, mathematical modeling, and tissue biopsies can be utilized.

D1 is expressed predominantly in liver and kidney, with lesser expression in the thyroid (12). D1 has a dual role, both producing a small amount of circulating $T_{3}$ (D1 is estimated to 
contribute approximately $24 \%$ of circulating $T_{3}$ in healthy individuals) and also preserving iodide by removing iodine from inactive metabolites of $T_{3}$ and $T_{4}$ in the liver and kidney (conjugated iodothyronines) (13). Of note, the D1 gene is upregulated by $T_{3}$, and thus in hyperthyroidism the proportion of circulating $T_{3}$ arising from the D1 pathway is significantly increased (11). Propylthiouracil (PTU) inhibits D1, explaining some of its therapeutic benefit in this setting (14).

D2 activity in humans has been detected in various tissues, predominantly in the central nervous system, pituitary gland, thyroid, heart, brown adipose tissue, and skeletal muscle (1517). The role of $\mathrm{D} 2$ is to convert $T_{4}$ to $T_{3}$; approximately $60 \%$ of circulating $T_{3}$ is estimated to originate from the D2 pathway in healthy individuals (18-20). Expressed in the endoplasmic reticulum (21,22), D2 generated $T_{3}$ first accumulates in the cell long enough to contribute to the nuclear $T_{3}$ pool (23); thus, changes in D2 activity can cause intracrine tissue-specific changes in thyroid signaling. Intracrine refers to the conversion of $T_{4}$ into $T_{3}$ within the cell resulting in a cell-specific modulation of the hormonal signaling. Subsequently, D2generated $T_{3}$ exits the cells, thus contributing to thyroid signaling in surrounding tissues (paracrine effects) and to the plasma pool of $T_{3}$ affecting thyroid signaling in other tissues. D1 on the other hand resides in the plasma membrane and does not seem to alter the intracellular $T_{3}$ concentration.

In healthy adults, D3 expression is found predominantly in the brain and skin $(24,25)$. In contrast to D1 and D2, D3 is an inactivating deiodinase, catalyzing the conversion of $T_{3}$ to $T_{2}$ $\left(3^{\prime}, 5^{\prime}-T_{2}\right)$, and $T_{4}$ to reverse $T_{3}\left(r T_{3}: 3,3^{\prime}, 5^{\prime}-T_{3}\right)$. Thus, the role of D3 is to clear $T_{3}$. Like D2, D3 is thought to have tissuespecific effects, preventing circulating $T_{3}$ from entering the nucleus. In disease states, and particularly in hypoxic states, D3 expression can be found ectopically in a wide range of tissues (26); the resultant increase in $T_{3}$ clearance promotes both tissue-specific and systemic lowering of $T_{3}$ and has been implicated in the pathogenesis of the "nonthyroidal illness syndrome" (27). Whether this is true tissue hypothyroidism or adaptation to illness is unclear $(28,29)$.

Thyroid hormone was long thought to cross the plasma membrane via simple diffusion, but recent studies have identified a number of thyroid hormone transporters including members of the monocarboxylate transporter family (MCT) and the organic anion transporting polypeptides (OATP), which are important for maintaining the intracellular concentrations of thyroid hormones (30). Thus, the particular circulating levels of thyroid hormones achieved during monotherapy, with higher serum $T_{4}$ and lower serum $T_{3}$ levels than the naturally euthyroid state (see section $7 b$ ), are available to the thyroid hormone transporters, which are then responsible for modulating intracellular levels of $T_{3}$. MCT and OATP transporters are expressed in liver, kidney, brain, and heart, with MCT8 and OATP1C1 being most important in the brain. Mutations identified in the thyroid hormone transporters constitute a clinically relevant group of mutations in the thyroid hormone signaling pathway (see section 13a).

\section{Discussion of the clinical literature}

Until the 1970s, the mainstay of thyroid hormone replacement therapy was desiccated thyroid extracts. Three factors were likely responsible for $\mathrm{LT}_{4}$ becoming the predominant therapy in the latter half of the 20th century: first, the isolation of $\mathrm{T}_{4}$ in 1927 by Kendall (31); second, the synthesis of $\mathrm{T}_{4}$ (32) and its better-absorbed sodium salt by Chalmers et al. (33); and third, the demonstration that the biologically active $\mathrm{T}_{3}$ was generated from $\mathrm{T}_{4}$ in humans (10).

Approximately $85 \mu \mathrm{g}$ of $\mathrm{T}_{4}$ is secreted by the thyroid gland daily. Of the total daily $\mathrm{T}_{3}$ production of about $33 \mu \mathrm{g}$ in normal man, approximately $80 \%$ (about $26 \mu \mathrm{g}$ ) arises from peripheral conversion from $\mathrm{T}_{4}$, and only about $20 \%$ (approximately $6.5 \mu \mathrm{g}$ ) derives from direct thyroidal secretion (34). It is now well established that while $T_{4}$ is the major secretory product of the thyroid gland, thyroid hormone action in peripheral tissues is due to the effects of $\mathrm{T}_{3}$ binding to its nuclear receptor, defining $\mathrm{T}_{4}$ as a pro-hormone for $\mathrm{T}_{3}$.

Gastrointestinal absorption of the tablet formulation of $\mathrm{LT}_{4}$ is in the $70 \%-80 \%$ range in healthy fasting adults (35). The long (approximately 7 day) half-life allows once-a-day dosing, and stable serum levels of both $\mathrm{T}_{4}$ and $\mathrm{T}_{3}$ (36). With such therapy, there will be a transient peak in serum $\mathrm{T}_{4}$ and free $(\mathrm{F}) \mathrm{T}_{4}$ levels of about $15 \%$ magnitude about 4 hours after $\mathrm{LT}_{4}$ administration (37). Moreover, in athyreotic individuals treated with $\mathrm{LT}_{4}$ to achieve a normal serum TSH, the serum $\mathrm{FT}_{4}$ is usually higher and the serum $\mathrm{T}_{3}$ is either normal $(38,39)$ or lower than the values seen in normal individuals (39-43). Steady-state levels of $\mathrm{T}_{4}$ and TSH are generally achieved in 6 weeks (approximately five to six half-lives) after initiation of therapy (38). Although there are no randomized clinical trials comparing $\mathrm{LT}_{4}$ to placebo to treat hypothyroidism, it has clearly been demonstrated that $\mathrm{LT}_{4}$ or $\mathrm{LT}_{3}$ withdrawal leads to a recurrence of hypothyroidism in patients who have undergone thyroid surgery for thyroid cancer or who were diagnosed with profound hypothyroidism (44-46).

\section{1b. What are the clinical and biochemical goals for levothyroxine replacement in primary hypothyroidism?}

\section{- RECOMMENDATION}

Levothyroxine replacement therapy has three main goals. These are (i) to provide resolution of the patients' symptoms and hypothyroid signs, including biological and physiologic markers of hypothyroidism, (ii) to achieve normalization of serum thyrotropin with improvement in thyroid hormone concentrations, and (iii) to avoid overtreatment (iatrogenic thyrotoxicosis), especially in the elderly.

\section{Strong recommendation. Moderate quality evidence.}

\section{Discussion of the clinical literature}

The goals of $\mathrm{LT}_{4}$ replacement in primary hypothyroidism are to achieve a state of euthyroidism and normalization of the circulating levels of TSH and thyroid hormones $(47,48)$. A state of euthyroidism is defined as the normalization of indices of thyroid hormone action and the absence or the regression of symptoms and clinical signs associated with hypothyroidism. The lack of specificity of hypothyroid symptoms and signs (49) and, particularly in case of autoimmune thyroid disease (AITD), the slow development of the pathologic state, renders difficult the assessment of the adequacy of the replacement therapy on a purely clinical basis. A randomized controlled trial (RCT) demonstrated that patients are unable to detect differences in the symptoms associated with hypothyroidism when the $\mathrm{LT}_{4}$ dose is changed by approximately $20 \%$. Of note, the change in dosage was sufficient to modify cholesterol levels (50). Similarly, patients may have no alteration in their hypothyroid symptom profile with 
Table 3. Main Properties and Roles of Iodothyronine Deiodinases Found in Human Tissues

\begin{tabular}{|c|c|c|c|}
\hline & $D 1$ & D2 & D3 \\
\hline $\begin{array}{l}\text { Position of iodine } \\
\text { in substrate molecule }\end{array}$ & $5^{\prime}$ or 5 & $5^{\prime}$ & 5 \\
\hline Preferred substrate & $\mathrm{rT}_{3}>\mathrm{T}_{4}$ & $\mathrm{~T}_{4}>\mathrm{rT}_{3}$ & $\mathrm{~T}_{3}>\mathrm{T}_{4}$ \\
\hline $\begin{array}{l}\text { Predominant tissue } \\
\text { localization }\end{array}$ & Liver, kidney, thyroid & $\begin{array}{l}\text { Pituitary gland, CNS, BAT, } \\
\text { skin, heart }\end{array}$ & $\begin{array}{l}\text { CNS, skin, placenta, } \\
\text { tumors }\end{array}$ \\
\hline Subcellular localization & Plasma membrane & Endoplasmic reticulum & $\begin{array}{l}\text { Plasma membrane, } \\
\text { nuclear membrane }\end{array}$ \\
\hline Hypothyroidism & $\downarrow$ & $\uparrow$ & $\downarrow$ \\
\hline Hyperthyroidism & $\uparrow$ & $\downarrow$ & $\uparrow$ \\
\hline $\mathrm{Km}\left(\mathrm{T}_{4}\right)$ & $2 \mu \mathrm{M}$ & $2 \mathrm{nM}$ & $37 \mathrm{nM}$ \\
\hline Selective activator & none & cAMP & Hypoxia/HIF-1 \\
\hline Selective inhibitor & PTU & none & none \\
\hline $\begin{array}{l}\text { Main role in thyroid } \\
\text { hormone economy } \\
\text { and signaling }\end{array}$ & $\begin{array}{l}\text { Contributes to approximately } \\
20 \% \text { of extrathyroidal } \\
\mathrm{T}_{3} \text { production in athyreotic } \\
\text { individuals; neutral } \\
\text { for intracellular } \mathrm{T}_{3} \text { levels }\end{array}$ & $\begin{array}{l}\text { Contributes to approximately } \\
80 \% \text { of extrathyroidal } \\
\mathrm{T}_{3} \text { production in athyreotic } \\
\text { individuals; increases } \\
\text { intracellular } \mathrm{T}_{3} \text { levels }\end{array}$ & $\begin{array}{l}\text { Main contributor } \\
\text { to the clearance of } \\
\mathrm{T}_{3} \text {; decreases intracellular } \\
\mathrm{T}_{3} \text { levels }\end{array}$ \\
\hline
\end{tabular}

BAT, brown adipose tissue; CNS, central nervous system; Km, substrate concentration at which the reaction rate is half maximum velocity; HIF-1, hypoxia-inducible factor $1 ; \mathrm{rT}_{3}$, reverse triiodothyronine; $\mathrm{T}_{3}$, triiodothyronine; $\mathrm{T}_{4}$, thyroxine.

changes in the $\mathrm{LT}_{4}$ dose that affect TSH and resting energy expenditure (51). TSH is the most reliable marker of adequacy of replacement treatment, and a value within the reference range (0.4-4.0 mIU/L) should be considered the therapeutic target.

Although no RCTs are currently available, a recent metaanalysis showed that significantly increased risk of cardiovascular mortality and morbidity was primarily observed in individuals with TSH levels $>10 \mathrm{mIU} / \mathrm{L}$, with potential effects of TSH values $>7 \mathrm{mIU} / \mathrm{L}(52)$. In an additional retrospective study, fewer ischemic heart disease events were associated with treatment of SCH in those under age 70 years (53). Thus, based on expectation of cardiovascular and other benefits, a target TSH in the age-specific reference range is generally recommended (see section 6a for a discussion of age-specific reference ranges). However, randomized trials are certainly needed before firm conclusions can be drawn. For example, it is certainly plausible that patients with worse cardiovascular disease might be less likely to be treated for subclinical hypothyroidism (for fear of exacerbating the cardiovascular disease) and have a worse overall clinical outcome. A target TSH in the lower tertile of the laboratory reference range, encompassing the median distribution of the values in the normal population (54), has also been proposed (55) and may in general be reasonable. However, this is not a universally endorsed approach (56). Moreover, a randomized cross-over study of 8 weeks' duration did not show changes in well-being or quality of life with TSH titration within the normal and subnormal range (50).

In some cases, $\mathrm{LT}_{4}$ alone may fail to restore the $\mathrm{T}_{3}$ levels to a value within the reference range in patients who have undergone total thyroidectomy and thus are devoid of residual endogenous production of thyroid hormone $(39,41,42)$. In euthyroid patients undergoing thyroidectomy and not requiring suppressive therapy, if $\mathrm{T}_{3}$ levels are chosen as one of the therapeutic targets, it is reasonable to titrate the therapy to achieve circulating levels of $\mathrm{T}_{3}$ similar to the presurgery values while maintaining the TSH value within the range of normality. Levels of $\mathrm{FT}_{4}$ above the reference range are often observed during replacement therapy with $\mathrm{LT}_{4}$ (39); no evidence yet exists indicating that this condition is associated with adverse events or poor outcome.

\section{1c. Are clinical parameters such as cold sensitivity and dry skin useful by themselves for assessing adequacy of levothyroxine replacement in primary hypothyroidism?}

\section{- RECOMMENDATION}

Although it may be helpful to follow changes in clinical symptoms longitudinally in patients treated for hypothyroidism, symptoms alone lack sensitivity and specificity and therefore are not recommended for judging adequacy of replacement in the absence of biochemical assessment. Therefore, symptoms should be followed but considered in the context of serum thyrotropin values, relevant comorbidities, and other potential causes.

\section{Weak recommendation. Low-quality evidence.}

\section{Discussion of the clinical literature}

The signs and symptoms associated with hypothyroidism are well known and include, but are not limited to, dry skin, cold intolerance, constipation, slowed thinking, weight gain, coarse skin, puffiness, slowed heart rate, and delayed relaxation of ankle reflexes. The symptoms often have an insidious onset and overlap significantly between patients with thyroid disease and those without. Many signs and symptoms of thyroid dysfunction are neither sensitive nor specific (57). For example, signs and symptoms associated with hypothyroidism such as dry skin, fatigue, and constipation may not reveal themselves with modest degrees of hypothyroidism and could have other causes. Several studies report a correlation between signs and symptoms of hypothyroidism and biochemical disease either based on an increasing cumulative number of symptoms (57) or on an abnormal clinical score (58). Unfortunately, while several signs or symptoms show relatively high specificity, such as puffiness and slowed movements, the sensitivity is relatively low. Physiological, psychological, and biochemical biomarkers of thyroid status, in addition to thyroid hormone levels, are available. Physiological parameters include heart rate, pulse wave arrival time $(59,60)$, echocardiographic parameters of left 
ventricular function, Achilles reflex time (58), voice fundamental frequency, and basal metabolic rate (61-63). To illustrate the lack of specificity, basal metabolic rate, although profoundly affected by extreme hypothyroidism, is also affected by other conditions such as fever, malignancy, and nutritional status $(64,65)$. Pulse wave arrival time can also be affected by atherosclerosis and systemic sclerosis. Achilles reflex time seems to have a reasonable sensitivity of $77 \%$ and a good specificity of $93 \%$ to confirm or exclude hypothyroidism (58), but it is limited by the fact that a small percentage of euthyroid individuals have an abnormal reflex time. Other clinical indices used to assess thyroid function include psychiatric and neuropsychological measures. Various rating scales have been used to assess the degree of hypothyroidism $(57,58,66-68)$. However, such clinical scores may not be sufficiently sensitive to signal subtle changes in thyroid status (69).

In summary, there are clearly signs and symptoms that are manifestations of untreated hypothyroidism, as documented in cross-sectional analyses. Resolution of symptoms can be documented even with treatment of mild hypothyroidism (70), but few studies document longitudinal monitoring of both symptoms and biochemical parameters within individual treated patients. Symptoms, therefore, are probably best followed within a framework of biochemical parameters, particularly serum TSH. Future research could follow symptoms longitudinally in treated patients in order to examine specificity and reliability of such symptoms in reflecting thyroid status. Another potential area for additional research is whether it is helpful to modify the $\mathrm{LT}_{4}$ dose because of residual signs and symptoms in a patient with a normal TSH, as long as the TSH remains within the goal reference range.

\section{1d. Are tissue markers of thyroid hormone action helpful in determining the adequacy of levothyroxine replacement in primary hypothyroidism?}

\section{- RECOMMENDATION}

Tissue biomarkers of thyroid hormone action are not recommended for routine clinical use, outside of the research setting, since these parameters are not sensitive, specific, readily available, or standardized.

\section{Weak recommendation. Low-quality evidence.}

\section{Mechanistic background}

Thyroid hormone action affects a wide range of biological pathways, and virtually all organ systems have a measurable response to the administration of replacement or supraphysiologic doses of $\mathrm{LT}_{4}(8,9)$. Measurement of thyroid hormoneresponsive gene expression is a means of assessing the impact of thyroid hormone on various tissues. The impact of the thyroidal secretion in a specific tissue depends on the amount of $T_{3}$ bound to the thyroid hormone receptor (TR) over time, with increasing amounts of $T_{3}$ establishing a greater thyroid hormone transcriptional "footprint" in any given tissue. This is because $T_{3}$ binding to TR modulates the expression of specific sets of $T_{3}$-responsive genes, constituting the basis of most biological effects of thyroid hormone. Multiple cell types, including hepatocytes, cardiac myocytes, skeletal myocytes, kidney cells, lung endothelial cells, and brain cells express genes that are exquisitely sensitive to regulation by thyroid hormone. This regulation may be positive or negative, depending on the particular gene and tissue. Thyroid hormone effects on rodent and cell models (71), in particular the rat hepatocyte (72) and cardiac myocyte (73), have been extensively studied. Measurement of gene expression in these tissues is not routinely used for clinical assessment of thyroid hormone status because it would require invasive procedures such as tissue biopsy, as was accomplished in a study of human skeletal muscle (74).

\section{Discussion of the clinical literature}

End-organ markers of response to thyroid hormone include sex hormone binding globulin (SHBG), osteocalcin, urinary ntelopeptides, total cholesterol, low-density lipoprotein (LDL) cholesterol, lipoprotein(a), creatine kinase, ferritin, myoglobin, and enzymes such as tissue plasminogen activator, angiotensin converting enzyme (ACE), and glucose 6-phosphate dehydrogenase (61,75-81). The results of RCTs have indicated that cholesterol $(50,82-86)$, and SHBG levels (50) are particularly affected by the administration of $\mathrm{LT}_{4}$. Similarly, RCTs have demonstrated that $\mathrm{LT}_{4}$ replacement therapy affects myocardial function (87) and particularly diastolic function (88-92), and, over time, the brachial artery intimal thickness $(89,93)$. Of note, the changes observed in these tissue markers of thyroid hormone action are often within the range of variance of the normal population, so these tests may be considered as an additional tool to allow a further optimization of the replacement therapy, once the TSH is already within target range. While resting energy metabolism and accurate millisecond timing of the Achilles' tendon relaxation phase have been historically used to assess the adequacy of replacement therapy, these methods are neither readily available nor well standardized. However, one study did suggest that resting energy expenditure was specifically altered by changes in $\mathrm{LT}_{4}$ dose, correlated well with TSH, and was more sensitive to dose alterations than either lipid profile, SHBG concentrations, or ACE levels (51).

\section{Choice of Levothyroxine Product}

\section{2a. Is there a clinical rationale for prescribing brand-name levothyroxine preparations in preference to generic levothyroxine? \\ - RECOMMENDATION}

Prescription of brand name levothyroxine, or alternatively maintenance of the same generic preparation (i.e., maintenance of an identifiable formulation of levothyroxine), is advised. Switches between levothyroxine products could potentially result in variations in the administered dose and should generally be avoided for that reason (see also recommendation 3d).

Weak recommendation. Low-quality evidence (for general populations)

Strong recommendation. Low-quality evidence (frail patients, high-risk thyroid cancer patients, pregnant patients)

Strong recommendation. Moderate-quality evidence (early childhood hypothyroidism)

\section{Discussion of the clinical literature}

A number of different brand name products and generic products are currently available. This literature review 
excluded studies on $\mathrm{LT}_{4}$ products available only outside of the United States. There is a long history of regulatory and clinical discussion regarding $\mathrm{LT}_{4}$ products $(94,95)$. The Food and Drug Administration (FDA) defines an $\mathrm{LT}_{4}$ formulation as equivalent if the $90 \%$ confidence interval of the difference of the pharmacokinetically derived areas under the curve (AUC) and difference of maximum concentrations (Cmax) of serum $\mathrm{T}_{4}$ fall within $80 \%-125 \%$ of a reference product when a comparable dose is administered to healthy volunteers. In this analysis, the $\mathrm{LT}_{4}$ dose is only tested at a single oral dose of $600 \mu \mathrm{g}$, and these results are extrapolated for all available dose strengths. There has been active discussion and controversy regarding this definition. This definition is problematic in that it does not require assessment of serum TSH, the commonly accepted marker of peripheral thyroid hormone action. Moreover, the dose of $\mathrm{LT}_{4}$ administered (i.e., $600 \mu \mathrm{g}$ ) is pharmacologic, and it cannot be assumed that similar kinetics apply to lower, more physiologic doses. Correction for the endogenous thyroid hormone serum levels seen in euthyroid individuals has only been recommended by the FDA since 2003 (96). The ATA, The Endocrine Society, and AACE issued a combined statement in 2004 expressing their concerns about the FDA's definition and its application to establishing bioequivalence of $\mathrm{LT}_{4}$ preparations (97).

Blakesley et al. (98), in a study funded by Abbott Laboratories, studied the FDA-recommended pharmacokinetic process of comparing $\mathrm{LT}_{4}$ products. Even when correction was made for endogenous serum $\mathrm{T}_{4}$ levels in healthy individuals, enabling the detection of differences in the administered dose of $25 \%$ and $33 \%$, they could not distinguish acute $\mathrm{LT}_{4}$ doses that differed by $12.5 \%$, leaving open the precise sensitivity of this method to exclude clinically meaningful differences in bioavailability between products considered equivalent.

It would seem more appropriate to determine bioequivalence of different $\mathrm{LT}_{4}$ preparations by assessing for comparable serum $\mathrm{T}_{4}, \mathrm{~T}_{3}$, and $\mathrm{TSH}$ levels after daily administration of the individual preparation for at least 4-6 weeks in athyreotic individuals. There are several studies of historical interest that have used a similar clinical protocol. Escalante et al. (99) studied two well-known, popular brands of $\mathrm{LT}_{4}$ (one of which has been reformulated twice since this evaluation) and found them to be both bioequivalent and therapeutically equivalent. Dong et al. (100), in a study sponsored by Knoll Pharmaceuticals, studied the pharmacokinetics profiles of two proprietary $\mathrm{LT}_{4}$ products (one of which has been reformulated since this evaluation) and two generic $\mathrm{LT}_{4}$ products (neither of which is currently available) and also concluded that they were bioequivalent and therapeutically equivalent. In contrast, Mayor et al. (101) of Knoll pharmaceuticals reanalyzed the data by Dong et al. (100) using correction for baseline $\mathrm{T}_{4}$ levels and the $\mathrm{TSH}$ data obtained during the trial, and found that the $\mathrm{LT}_{4}$ products that seemed to be pharmacokinetically bioequivalent were, in fact, not equivalent therapeutically. No prospective studies assessing this question have focused on bioequivalence as well as clinically therapeutic effectiveness in currently available products. However, there is likely an important difference between bioequivalence, which indicates meeting the FDA regulation, and clinical equivalence, which indicates comparability of $\mathrm{FT}_{4}, \mathrm{~T}_{3}$, and $\mathrm{TSH}$ concentrations, as well as clinical parameters. Simulation studies also support the use of
TSH-based protocols for judging bioequivalence, rather than using pharmacokinetic studies (102).

Since 2007 the FDA has required that $\mathrm{LT}_{4}$ preparations maintain $95 \%-105 \%$ of their stated potency, revised from a prior requirement of $90 \%-110 \%$, throughout their shelf life $(103,104)$. Furthermore, the FDA has required that all $\mathrm{LT}_{4}$ products be reassessed as though they were new drugs (105). When a generic or branded $\mathrm{LT}_{4}$ preparation meets the criteria noted above for bioequivalence and potency, the FDA has determined that $\mathrm{LT}_{4}$ preparations can be substituted for one another by the pharmacy, unless specifically designated to be dispensed as written by the prescriber. In their joint statement, referred to previously, the three different endocrine societies recommend repeat thyroid function testing when a patient is switched from one $\mathrm{LT}_{4}$ preparation to another to ensure the goal TSH (and $\mathrm{FT}_{4}$ and $\mathrm{T}_{3}$ ) concentrations are achieved (97). Although, not based on specific studies of the utility of this approach, this would appear to be a clinically appropriate, "common sense" recommendation. It is recommended that once the therapeutic target is reached, the patient should remain on the same dose and preparation of medication to the extent possible.

In light of this discussion, the clinical question remains "Is there a clinical rationale for prescribing brand-name $\mathrm{LT}_{4}$ preparations in preference to generic $\mathrm{LT}_{4}$ ?" This issue would not arise if a patient could be maintained on an identifiable formulation of $\mathrm{LT}_{4}$ (whether brand or generic). In other words, if a patient could be prescribed the same generic formulation from refill to refill, there would be no reason to be concerned about distinction between branded and generic products. However, if prescribing a generic product exposes the patient to the vagaries of the pharmacy and insurance system that permits switches between generic products, then a potential concern remains. Any recommendation is inherently based, at least to some extent, on expert opinion. It seems reasonable for a patient to remain on a given $\mathrm{LT}_{4}$ product as long as possible, and if a change in product is made then thyroid function tests should be rechecked. In patients with thyroid cancer, especially when a desired goal TSH is relevant for curtailing possible disease recurrence or progression, it is important to maintain $\mathrm{LT}_{4}$ product stability. A recent retrospective study of children treated for congenital hypothyroidism $(\mathrm{CH})$ exclusively with either brand-name or generic $\mathrm{LT}_{4}$ noted no difference in clinical outcomes (106). However, a prospective, randomized trial, also conducted in the pediatric population being treated for hypothyroidism, showed that similar doses of a branded product and a generic considered by the FDA to be interchangeable did not result in comparable TSH values (107). In this study, 30 children with primary hypothyroidism (20 with $\mathrm{CH}$; 11 with autoimmune thyroiditis) and an initial TSH greater than $100 \mathrm{mIU} / \mathrm{L}$ were studied. Following 8 weeks of brand name $\mathrm{LT}_{4}$, the serum TSH was statistically significantly lower $(p=0.002)$ as compared to 8 weeks of generic $\mathrm{LT}_{4}$. This difference was still observed following adjustment for age. Thyroid hormone levels were not statistically different. In further analysis, the TSH difference was restricted to the patients with $\mathrm{CH}$. The authors concluded that branded $\mathrm{LT}_{4}$ was not bioequivalent to an $\mathrm{AB}$-rated generic $\mathrm{LT}_{4}$ preparation. The authors stated "It would therefore seem prudent not to substitute $\mathrm{LT}_{4}$ formulations in patients with severe $\mathrm{CH}$, particularly in those $<3$ years of age. Our results may have important implications for other severely hypothyroid patients in whom 
precise titration of $\mathrm{LT}_{4}$ is necessary." In general, aspects of the study design, including its prospective nature, give credence to the results of the latter study (108).

The recommendation for maintaining a particular identifiable formulation of $\mathrm{LT}_{4}$ is not based on an anticipated inherent superiority of one particular $\mathrm{LT}_{4}$ product, but is instead based on the concern that even products judged to be bioequivalent do not have therapeutic equivalence, and that switching of products could lead to perturbations in serum TSH. This is a particular concern in frail patients, those with thyroid cancer, and the pediatric age group. This issue of bioequivalence is controversial and requires further study. Clinical trials should include comparisons of branded $\mathrm{LT}_{4}$ products not only to various generic formulations, but should also compare brand $\mathrm{LT}_{4}$ and generic formulations to themselves over time.

\section{2b. Are there situations in which therapy with levothyroxine dissolved in glycerin and supplied in gelatin capsules may have advantages over standard levothyroxine?}

\section{- RECOMMENDATION}

Although there are preliminary small studies suggesting that levothyroxine dissolved in glycerin and supplied in gelatin capsules may be better absorbed than standard levothyroxine in selected circumstances such as concomitant use of proton pump inhibitors or concomitant coffee consumption, the present lack of controlled long-term outcome studies does not support a recommendation for the use of such preparations in these circumstances. Switch to a gel capsule might be considered in the rare case of putative allergies to excipients.

\section{Weak recommendation. Low-quality evidence.}

\section{Discussion of the clinical literature}

An oral gel preparation that contains only $\mathrm{LT}_{4}$, glycerin, gelatin, and water has been developed. The gelatin capsule contains $\mathrm{LT}_{4}$ as a liquid (109). This formulation is in contrast to standard solid $\mathrm{LT}_{4}$ tablets that contain excipients and dyes. Pabla et al. (110) studied in vitro dissolution of gel capsules and noted rapid and efficient dissolution at low $\mathrm{pH}$ concentrations. Colucci et al. (111) observed that gel capsules met the revised FDA potency guidelines of 95\%-105\% $(103,104)$ and also met equivalence criteria. At present, there are no prospective, randomized clinical trials in hypothyroid individuals comparing gel capsules to other commercial $\mathrm{LT}_{4}$ preparations in terms of the serum $\mathrm{FT}_{4}, \mathrm{~T}_{3}$, and TSH concentrations achieved during chronic therapy.

There is some evidence that gel capsules are absorbed preferentially in selected circumstances, such as during consumption with coffee and during use of proton pump inhibitors (PPIs). However, four of the studies that have examined this issue are published only in abstract form, and therefore this discussion and recommendation is based on three peerreviewed publications only. Use of gel capsules, instead of standard $\mathrm{LT}_{4}$, allowed suppression of serum TSH during chronic therapy in patients with thyroid disease that could not be achieved by ingestion of standard $\mathrm{LT}_{4}$ with Italian style coffee (112). An uncontrolled retrospective study noted lower serum TSH concentrations in patients who were switched from $\mathrm{LT}_{4}$ tablets to the same dose of $\mathrm{LT}_{4}$ as a soft gel capsule (113).
A case report describes a patient taking a PPI whose serum TSH was higher during treatment with $\mathrm{LT}_{4}$ tablets than with the same dose given as a gel capsule. An absorption study showed better absorption of the $\mathrm{LT}_{4}$ from the gel capsule (114). Although no specific definitive recommendation can be made with regard to the use of gel capsules in patients with hypothyroidism because of the lack of peer-reviewed publications, preliminary evidence from retrospective studies suggests that it may have a more favorable absorption profile compared with standard $\mathrm{LT}_{4}$ tablets. An alternative approach to switching to a soft gel capsule, in situations of suspected poor absorption, is to increase the dose of the $\mathrm{LT}_{4}$ tablet with periodic monitoring of thyroid function tests.

A liquid formulation of $\mathrm{LT}_{4}$ that is packaged in polyethylene ampoules, and that is not available within the United States, has been suggested, based on case reports or uncontrolled studies only, to be better absorbed than tablets if there is malabsorption following bariatric surgery or if $\mathrm{LT}_{4}$ is consumed with breakfast $(115,116)$. When the liquid $\mathrm{LT}_{4}$ preparation contained in these ampoules is added to breakfast beverages the $\mathrm{T}_{4}$ content is stable for at least 20 minutes (117).

There is the theoretical possibility that gel capsules or liquid $\mathrm{LT}_{4}$ would be useful in patients with allergies to any of the excipients in solid $\mathrm{LT}_{4}$ tablets.

\section{Levothyroxine Absorption and Metabolism}

\section{3a. How should levothyroxine administration be timed with respect to meals and beverages in order to maintain maximum, consistent absorption?}

\section{- RECOMMENDATION}

Because co-administration of food and levothyroxine is likely to impair levothyroxine absorption, we recommend that, if possible, levothyroxine be consistently taken either 60 minutes before breakfast or at bedtime ( 3 or more hours after the evening meal) for optimal, consistent absorption.

Weak recommendation. Moderate quality of evidence.

\section{Discussion of the clinical literature}

Food, dietary fiber, and beverages. Absorption of $\mathrm{LT}_{4}$ occurs in the jejunum and ileum (118). An acidic $\mathrm{pH}$ in the stomach, as occurs during fasting conditions, appears to be important for subsequent intestinal absorption. The absorption of an orally administered dose of $\mathrm{LT}_{4}$ is about $70 \%-80 \%$ under optimum fasting conditions $(38,119,120)$. Therefore, if a patient is unable to take oral medications, the appropriate intravenous dose is approximately $75 \%$ of the oral dose.

Seven studies regarding co-administration with foods and beverages in adults were identified based on literature review (120-126). It should be noted that only one of these is a randomized study (125), thus limiting the ability to draw firm conclusions from the data. When $\mathrm{LT}_{4}$ is co-administered with food, absorption is reduced compared with absorption in the fasting state. In one study using double isotope methods in healthy volunteers, which is limited by the participants not serving as their own controls, the documented $\mathrm{LT}_{4}$ absorption with food was determined to be $64 \%$ compared with $80 \%$ in the fasting state (120). In another case report, four patients who had failure to suppress their TSH values while consuming their 
breakfast within 20 minutes of their $\mathrm{LT}_{4}$ ingestion underwent absorption testing (121). The ingestion of food and $\mathrm{LT}_{4}$ in close proximity to each other was associated with an $\mathrm{LT}_{4}$ absorption curve that was characterized by delayed absorption. After a 1-month period of delaying breakfast by 60 minutes, the patients were seen to achieve suppression of their TSH values, suggesting correction of the impaired absorption, although absorption testing was not repeated to confirm this.

Case reports also show that fiber (123) and soy products (124) appear to be associated with impaired $\mathrm{LT}_{4}$ absorption. However, fiber did not appear to affect absorption in a study of $\mathrm{LT}_{4}$ in healthy volunteers (127). A summary of several case studies of increased TSH values in hypothyroid patients ingesting their $\mathrm{LT}_{4}$ with espresso coffee has been reported (122). Absorption studies performed in these patients and in volunteers support the role of coffee in reducing $\mathrm{LT}_{4}$ absorption. Compared with water, coffee reduced both the maximum concentration $(\mathrm{Cmax})$ and the AUC documented during these absorption studies. In vitro binding studies performed by the same investigators (122) suggested, as has been previously reported with fiber (123), that espresso coffee acts to sequester $\mathrm{LT}_{4}$, and so it may have this action within the intestine. Grapefruit juice, which increases the plasma concentrations of several drugs that are substrates for CYP3A4 and possibly inhibits intestinal uptake transporters, was not found to substantially affect $\mathrm{LT}_{4}$ absorption in a randomized cross-over study of 10 healthy subjects (125).

Timing of levothyroxine administration. Four studies $(121,126,128,129)$, out of the six studies examining this issue (121,128-131), have shown that the timing of $\mathrm{LT}_{4}$ administration influences the serum TSH values of hypothyroid patients receiving treatment. Three of these had a cross-over design and thus are the most rigorous studies. The previously mentioned case study from 1995 examined four patients in whom TSH suppression therapy was difficult to achieve while they were eating breakfast 15-20 minutes after $\mathrm{LT}_{4}$ ingestion (121). Consuming breakfast within 20 minutes after taking $\mathrm{LT}_{4}$ resulted in a higher TSH, compared with when these same patients had breakfast 60 minutes after taking their $\mathrm{LT}_{4}$, suggesting impaired absorption with a separation of less than 60 minutes. In contrast, a retrospective study of elderly nursing home residents showed that serum TSH values were unaffected when the timing of $\mathrm{LT}_{4}$ administration was changed from 9 am (1-2 hours after breakfast) compared with midnight ( 3 hours after a snack) (130). Presumably, both these conditions qualified as postprandial. Another parallel design study, in which patients newly diagnosed with hypothyroidism were prescribed $\mathrm{LT}_{4}$ either 30 minutes before breakfast or in the evening, showed that at 12 weeks the achieved TSH was $5.13 \mathrm{mIU} / \mathrm{L}$ (standard deviation 9.36) with morning dosing versus $3.27 \mathrm{mIU} / \mathrm{L}$ (standard deviation $4.19 \mathrm{mIU} / \mathrm{L}$ ) with evening dosing (131). These two results were not statistically different. However, the study utilized two separate groups of patients who were hypothyroid, with serum TSH values of approximately $80 \mathrm{mIU} / \mathrm{L}$ at the start of the study, and thus not at steady state.

Two recent cross-over studies had different findings with the lowest serum TSH values being seen either 1 hour before breakfast or at bedtime $(128,129)$. In one of these studies, patients took their $\mathrm{LT}_{4}$ either at least 1 hour before break- fast, with breakfast, or at bedtime for 8 weeks. The mean TSH values seen with these different conditions were 1.06, 2.93 , and $2.19 \mathrm{mIU} / \mathrm{L}$ respectively (128). In the other study, which incorporated a double-blind design, patients took either $\mathrm{LT}_{4}$ or placebo capsules 30 minutes before breakfast and at bedtime for 12 weeks of each regimen. TSH values were 0.9-1.6 mIU/L higher with the morning compared with the bedtime $\mathrm{LT}_{4}$ ingestion (129). A third cross-over study found higher, more variable $\mathrm{TSH}$ values when $\mathrm{LT}_{4}$ was consumed with breakfast, compared with when it was taken 60 minutes before breakfast (126), although TSH values remained within the reference range even with the nonfasting regimen. If the results of these studies are consolidated, with lower TSH values being equated with better absorption, the conditions associated with better absorption (ordered from best to most impaired) are 60 minutes before breakfast, bedtime, 30 minutes before breakfast, and with breakfast. The fasting regimen has the additional advantage of producing more consistent TSH values compared with the bedtime regimen.

To put the summarized data in context, it is important to consider not only when $\mathrm{LT}_{4}$ absorption is optimal, but also to be aware of what timing promotes adherence. For example, although a fasting regimen may promote absorption, it may have the disadvantage of being maximally inconvenient for patients. Thus, a patient's schedule and preference should be taken into account and if consumption of $\mathrm{LT}_{4} 1$ hour before breakfast is not feasible, a bedtime regimen may be the next best choice. Another regimen that is consistently maintained, such as 30 minutes before breakfast, may also be reasonable. For this latter regimen to maintain a stable serum $\mathrm{TSH}$, it would probably also be important to consume a breakfast with similar daily food choices and avoid foods that are most noted for interfering with $\mathrm{LT}_{4}$ absorption. It is important to remember that no long-term studies have examined adherence with the various timing regimens, the consequences of variability in serum TSH values, or outcomes with different timing of $\mathrm{LT}_{4}$ ingestion.

\section{3b. Are there medications and supplements that should not be co-administered with levothyroxine in order to avoid impaired absorption?}

\section{- RECOMMENDATION}

We recommend that where feasible, levothyroxine should be separated from other potentially interfering medications and supplements (e.g., calcium carbonate and ferrous sulfate). A 4-hour separation is traditional, but untested. Other medications (e.g., aluminum hydroxide and sucralfate) may have similar effects, but have been insufficiently studied.

\section{Weak recommendation. Weak quality evidence.}

\section{Discussion of the clinical literature}

The literature review revealed 20 relevant articles. Studies documenting altered $\mathrm{LT}_{4}$ absorption in animals were not included. The list of medications that can alter the absorption of $\mathrm{LT}_{4}$ is extensive and includes calcium carbonate (132-134), PPIs (135), bile acid sequestrants (cholestyramine and colesevelam) $(136,137)$, phosphate binders $(138,139)$, ferrous sulfate $(140,141)$, aluminum-containing antacids $(142,143)$, 
and sucralfate $(144,145)$. Only two of these medications have been studied in a cross-over study design of chronic therapy $(132,145)$, with two other studies also conducted prospectively $(140,143)$. The effects of calcium carbonate and ferrous sulfate were shown in prospective, but nonrandomized, noncontrolled trials. The prospective study of aluminum hydroxide was performed in only five patients. The study of sucralfate was randomized and controlled, but was carried out in only nine patients. Moreover, many of these effects have been documented in individual case reports only.

Hypothyroid patients with normal TSH concentrations were asked to take their $\mathrm{LT}_{4}$ with calcium carbonate for several months. Their serum TSH values increased from a mean of $1.6 \mathrm{mIU} / \mathrm{L}$ to $2.7 \mathrm{mIU} / \mathrm{L}$, and returned to $1.4 \mathrm{mIU} / \mathrm{L}$ after discontinuation of the calcium (132). In vitro binding studies suggested adsorption of $\mathrm{LT}_{4}$ to calcium at acidic $\mathrm{pH}$ levels. A subsequent absorption study showed that both the Cmax and AUC were reduced when calcium carbonate and $\mathrm{LT}_{4}$ were co-administered (133). Calcium carbonate, citrate, and acetate all appear to have similar effects, with absorption studies in healthy volunteers showing a decrement in absorption of approximately $20 \%$ (134).

In a retrospective analysis, initiation of therapy with a PPI was associated with an increase in the serum TSH values of $\mathrm{LT}_{4}$-treated patients, compared with a control group in whom such therapy was not initiated (135). LT $_{4}$ absorption was unaltered with use of PPIs $(146,147)$ and histamine-2 blockers (147) for a 1-week period in two other studies in healthy volunteers. These conflicting findings may suggest that only chronic oral therapy is associated with decreased $\mathrm{LT}_{4}$ absorption.

Two studies have implicated bile acid sequestrants as being responsible for binding $\mathrm{LT}_{4}$ within the intestine $(136,137)$. Both of these reports incorporated pharmacokinetic studies in healthy volunteers showing reduced absorption with co-administration of the agent and $\mathrm{LT}_{4}$. The effects of the phosphate binder sevelamer has been examined in both a retrospective study (139) and a pharmacokinetic study (138). In the retrospective analysis, patients taking this agent needed higher $\mathrm{LT}_{4}$ doses to normalize their serum TSH than patients taking calcium products as phosphate binders (139). The pharmacokinetic study showed decreased $\mathrm{LT}_{4}$ absorption when these agents were coadministered (138).

The effect of ferrous sulfate has been examined in a trial (140) and a case report (141). During the trial, the TSH values of 14 hypothyroid patients increased from a mean of $1.6 \mathrm{mIU} / \mathrm{L}$ to a mean of $5.4 \mathrm{mIU} / \mathrm{L}$ with simultaneous ingestion of ferrous sulfate and $\mathrm{LT}_{4}$. A need for increased $\mathrm{LT}_{4}$ while taking ferrous sulfate was observed in the case report (141). Following documentation of increased $\mathrm{LT}_{4}$ requirement in a patient taking an aluminum-containing antacid (142), the same investigators showed an increase in TSH levels in five patients who were asked to take aluminum hydroxide with their $\mathrm{LT}_{4}$ (143). Studies of sucralfate are consistent with impaired absorption $(144,145)$. Both a case report of elevated TSH levels and an absorption study in healthy volunteers showing decreased and delayed $\mathrm{LT}_{4}$ absorption are consistent with binding of $\mathrm{LT}_{4}$ by sucralfate (144). A blinded cross-over study of nine patients taking $\mathrm{LT}_{4}$ failed to show a statistically significant difference in TSH while patients were taking either placebo or sucralfate in addition to their $\mathrm{LT}_{4}$, although the study may have been underpowered and the mean serum TSH values for the placebo and sucralfate groups were 2.69 and $4.63 \mathrm{mIU} / \mathrm{L}$, respectively (145).

Although the impact of multivitamins on $\mathrm{LT}_{4}$ absorption does not appear to have been studied, their calcium and ferrous salt content would be expected to result in impaired absorption. Based on this supposition they are included in lists of medications potentially impairing $\mathrm{LT}_{4}$ absorption (3). Effects of several additional medications have been documented in individual cases reports (148-151). These data regarding the effect of medications on $\mathrm{LT}_{4}$ absorption are summarized in Tables 4 and 5. Table 4 summaries pharmacokinetics studies, while Table 5 shows the trials and case reports.

\section{3c. Are there gastrointestinal conditions that should be considered when a patient's levothyroxine dose is much higher than expected?}

\section{- RECOMMENDATION}

In patients in whom levothyroxine dose requirements are much higher than expected, evaluation for gastrointestinal disorders such as Helicobacter pylori-related gastritis, atrophic gastritis, or celiac disease should be considered. Furthermore, if such disorders are detected and effectively treated, re-evaluation of thyroid function and levothyroxine dosage is recommended.

Strong recommendation. Moderate quality of evidence.

\section{Discussion of the clinical literature}

Literature review identified 18 studies addressing this topic. Several gastrointestinal disorders appear to affect either $\mathrm{LT}_{4}$ absorption or serum TSH levels, possibly mediated through an impact on gastric acidity. These effects have been shown in retrospective studies, although reduction of $\mathrm{LT}_{4}$ dosage requirement with treatment of Helicobacter pylori gastritis and celiac disease has been documented prospectively.

In a prospective, nonrandomized study treatment of $H$. pylori was associated with reduction of serum TSH levels from 30.5 to $4.2 \mathrm{mIU} / \mathrm{L}$ in patients who were considered nonresponsive to high doses of $\mathrm{LT}_{4}$ (152). In another study, comparison of patients with gastritis, related either to $H$. pylori or atrophic gastritis, with a reference group without gastric disorders showed that the daily $\mathrm{LT}_{4}$ requirement was up to $34 \%$ higher in those with gastritis ( $2.05 \mathrm{vs} .1 .5 \mu \mathrm{g} / \mathrm{kg} / \mathrm{d}$ ) (153). Furthermore, eradication of $H$. pylori infection and initiation of omeprazole were associated with decreased and increased TSH values, respectively. In addition, in patients receiving $\mathrm{LT}_{4}$ therapy, the magnitude of their $\mathrm{LT}_{4}$ requirement was correlated with the presence or absence of serum parietal cell antibodies (154). Higher $\mathrm{LT}_{4}$ doses were required in those with positive antibodies, and the $\mathrm{LT}_{4}$ dose was positively associated with the antibody titer and the severity of the gastritis. Autoimmune atrophic gastritis is particularly prevalent in older patients with Hashimoto's thyroiditis and hypothyroidism. In one study, $45 \%$ of patients older than age 60 with Hashimoto's thyroiditis also had chronic atrophic gastritis (155).

Celiac disease is also more common in patients with underlying AITD. Two retrospective studies documented higher $\mathrm{LT}_{4}$ requirements in patients with celiac disease, 
Table 4. Medications Affecting Levothyroxine Absorption, as Shown in Pharmacokinetic Studies

\begin{tabular}{|c|c|c|c|c|c|}
\hline Medications & Type of study & $\begin{array}{l}\text { Hypothyroid } \\
\text { versus healthy } \\
\text { subjects }\end{array}$ & No. of subjects & $\begin{array}{l}\text { Pharmacokinetic } \\
\text { study showing } \\
\text { reduced absorption? }\end{array}$ & Reference \\
\hline $\begin{array}{l}\text { Calcium } \\
\text { carbonate }\end{array}$ & $\begin{array}{l}\text { Randomized, cross-over } \\
\text { absorption study }\end{array}$ & Healthy & 7 & $\begin{array}{l}\text { Yes, maximum absorption } \\
84 \% \text { vs. } 58 \%\end{array}$ & 133 \\
\hline $\begin{array}{l}\text { Calcium } \\
\text { carbonate, } \\
\text { citrate, acetate }\end{array}$ & $\begin{array}{l}\text { Four-period absorption } \\
\text { study with random } \\
\text { cross-over to calcium } \\
\text { preparations }\end{array}$ & Healthy & 8 & $\begin{array}{l}\text { Yes, } \mathrm{T}_{4} \text { response } \\
\text { area with calcium } \\
75 \%-81 \% \\
\text { (100\% without) }\end{array}$ & 134 \\
\hline PPI (pantoprazole) & $\begin{array}{l}\text { Crossover absorption } \\
\text { study after } 1 \text { week } \\
\text { of PPI versus no PPI }\end{array}$ & Healthy & $\begin{array}{l}20 \text { (10 no } \\
\text { PPI first; } \\
10 \text { PPI first })\end{array}$ & $\begin{array}{l}\text { No, } \mathrm{T}_{4} \text { AUC unaffected } \\
\text { by PPI }\end{array}$ & 146 \\
\hline $\begin{array}{l}\text { PPI (pantoprazole) } \\
\text { and H2-blocker } \\
\text { (famotidine) }\end{array}$ & $\begin{array}{l}\text { Randomized to absorption } \\
\text { study with drug after } \\
\text { absorption study without }\end{array}$ & Healthy & 20 & $\begin{array}{l}\text { No, } \mathrm{T}_{4} \text { peak and } \mathrm{T}_{4} \text { AUC } \\
\text { unaffected by PPI } \\
\text { and } \mathrm{H} 2 \text {-blocker }\end{array}$ & 147 \\
\hline Cholestyramine & $\begin{array}{l}\text { Six absorption studies } \\
\text { each in five healthy } \\
\text { participants }\end{array}$ & Healthy & 5 & $\begin{array}{l}\text { Yes, best absorption } \\
\text { with } 5 \mathrm{~h} \text { separation } \\
\text { of drug and } \mathrm{LT}_{4}\end{array}$ & 136 \\
\hline Colesevelam & $\begin{array}{l}\text { Nonrandomized, } \\
\text { cross-over study } \\
\text { with and without drug }\end{array}$ & Healthy & 6 & $\begin{array}{c}\text { Yes, response area } 3.8 \% \\
\text { with (100\% without) }\end{array}$ & 137 \\
\hline Selevamer & $\begin{array}{l}\text { Randomized to absorption } \\
\text { study with drug after } \\
\text { absorption study without }\end{array}$ & Healthy & 7 & $\begin{array}{l}\text { Yes, } \mathrm{T}_{4} \text { response area } 50 \% \\
\text { with selevamer } \\
\text { (100\% without) }\end{array}$ & 138 \\
\hline Sucralfate & $\begin{array}{l}\text { Absorption study with } \\
\text { and without drug }\end{array}$ & Healthy & 5 & $\begin{array}{l}\text { Yes, reduced and delayed } \\
\text { peak absorption }\end{array}$ & 144 \\
\hline Raloxifene & $\begin{array}{l}\text { Absorption study with } \\
\text { and without drug }\end{array}$ & Hypothyroid & 1 & $\begin{array}{l}\text { Yes, lower peak } \mathrm{T}_{4} \text { levels } \\
\text { with raloxifene }\end{array}$ & 148 \\
\hline
\end{tabular}

AUC, area under the curve; H2-blocker, acid blocker; $\mathrm{LT}_{4}$, levothyroxine; PPI, proton pump inhibitor.

compared with unaffected hypothyroid patients $(156,157)$. Institution of a gluten-free diet reduced the requirement. There are also case reports of increased $\mathrm{LT}_{4}$ requirements in individuals with celiac disease $(158,159)$, lactose intolerance (160), and intestinal giardiasis (161) with reduction in the $\mathrm{LT}_{4}$ requirement occurring after institution of a gluten-free or lactose-restricted diet and treatment of the infection, respectively. Data comparing the magnitude of increased $\mathrm{LT}_{4}$ requirement in these different gastrointestinal conditions appear to be lacking. However, dramatic increases in serum TSH appear to be possible in patients with more than one factor contributing to impaired $\mathrm{LT}_{4}$ absorption, as occurred in a patient with both celiac disease and calcium carbonate use (162).

In addition to the gastrointestinal conditions discussed above, clinicians should be alert for other conditions that may affect $\mathrm{LT}_{4}$ requirement. Examples of factors that are also associated with decreased absorption are advancing age (35) and extreme obesity (body mass index $[\mathrm{BMI}]>40 \mathrm{~kg} / \mathrm{m}^{2}$ ) (163). Although there are case reports of increased $\mathrm{LT}_{4}$ requirements after intestinal bypass surgery (164-166), when studied directly $\mathrm{LT}_{4}$ absorption appeared to be preserved after Roux-en-Y surgery (167) and in 32 patients undergoing various other gastric bypass procedures (168). Such reports are consistent with the ileum being the main site of $\mathrm{LT}_{4}$ absorption.

\section{3d. Are different levothyroxine products associated with different absorption, such that a switch to a different brand name or generic is associated with a significantly different serum TSH?}

\section{- RECOMMENDATION}

Because use of different levothyroxine products may sometimes be associated with altered serum TSH values, a change in an identifiable formulation of levothyroxine (brand name or generic) should be followed by re-evaluation of serum TSH at steady state.

\section{Weak recommendation. Low-quality evidence.}

\section{Discussion of the clinical literature}

Assuming that their potency is similar and adherence is similarly maintained, if different identifiable formulations of $\mathrm{LT}_{4}$ of the same dose result in different serum $\mathrm{TSH}$ values during chronic therapy, this is likely to be due to different absorption. A simulation study in which either $\mathrm{LT}_{4}$ tablet potency or absorption were varied showed that altered absorption could result in achievement of different TSH values (102). Substitution of $\mathrm{LT}_{4}$ preparations potentially having different absorption characteristics has, historically, lead to changes in serum TSH in some studies (101), but in other studies it has not 
Table 5. Medications Reducing Levothyroxine Absorption, as Shown in Trials and Case Studies in Hypothyroid Patients

\begin{tabular}{|c|c|c|c|c|c|}
\hline Medications & Type of study & $\begin{array}{l}\text { No. } \\
\text { of subjects }\end{array}$ & $\begin{array}{l}\text { TSH values mIU/L } \\
\text { (w/o medication, } c / w \\
\text { with medication) }\end{array}$ & Binding study? & Reference \\
\hline $\begin{array}{l}\text { Calcium } \\
\text { carbonate }\end{array}$ & $\begin{array}{l}\text { Prospective, } \\
\text { cross-over trial }\end{array}$ & 20 & 1.6 vs. 2.7 & $\begin{array}{l}\text { Yes, } \mathrm{LT}_{4} \text { adsorbs } \\
\text { to calcium }\end{array}$ & 132 \\
\hline $\begin{array}{l}\text { PPI } \\
\text { (lansoprazole) }\end{array}$ & $\begin{array}{l}\text { Retrospective chart } \\
\text { review of PPI } \\
\text { initiation }\end{array}$ & $\begin{array}{l}55 \text { controls, } \\
37 \text { taking PPI }\end{array}$ & $\begin{array}{l}\text { Increased by } 0.11 \\
\text { vs. } 0.69\end{array}$ & $\mathrm{n} / \mathrm{a}$ & 135 \\
\hline Cholestyramine & Case reports & 2 & $\begin{array}{l}\text { Increased while taking } \\
\text { cholestyramine }\end{array}$ & $\begin{array}{l}\text { Yes, cholestyramine } \\
\text { bound to } \mathrm{LT}_{4}\end{array}$ & 136 \\
\hline Selevamer & $\begin{array}{l}\text { Retrospective } \\
\text { chart review }\end{array}$ & 67 & $\begin{array}{l}\text { Mean TSH } 20 \text { with } \\
\text { selevamer }\end{array}$ & $\mathrm{n} / \mathrm{a}$ & 139 \\
\hline Ferrous sulfate & $\begin{array}{l}\text { Nonrandomized, } \\
\text { prospective trial }\end{array}$ & 14 & TSH 1.6 vs 5.4 & $\begin{array}{l}\text { Yes, } \mathrm{T}_{4} \text { formed } \\
\text { a complex with iron }\end{array}$ & 140 \\
\hline Ferrous sulfate & $\begin{array}{l}\text { Case report (patient } \\
\text { also pregnant } \\
\text { and post partum) }\end{array}$ & 1 & TSH 1.3 c/w 29 & & 141 \\
\hline $\begin{array}{l}\text { Aluminum- } \\
\text { containing } \\
\text { antacid }\end{array}$ & Case report & 1 & TSH 1.1 vs. 36 & $\mathrm{n} / \mathrm{a}$ & 142 \\
\hline $\begin{array}{l}\text { Aluminum- } \\
\text { containing } \\
\text { antacid }\end{array}$ & $\begin{array}{l}\text { Nonrandomized, } \\
\text { prospective study }\end{array}$ & 5 & $\begin{array}{l}\text { TSH increased } \\
\text { from } 2.6 \text { to } 7.2\end{array}$ & $\begin{array}{l}\text { Yes, } \mathrm{T}_{4} \text { adsorbed } \\
\text { to antacid }\end{array}$ & 143 \\
\hline Sucralfate & Case report & 1 & $\begin{array}{l}\text { TSH increased from } \\
\text { normal to } 30\end{array}$ & $\mathrm{n} / \mathrm{a}$ & 144 \\
\hline Sucralfate & $\begin{array}{l}\text { Placebo-controlled, } \\
\text { randomized }\end{array}$ & 9 & TSH 2.7 vs. 4.6 & $\mathrm{n} / \mathrm{a}$ & 145 \\
\hline Raloxifene & Case report & 1 & TSH normal vs. 9.4 & $\mathrm{n} / \mathrm{a}$ & 148 \\
\hline Orlistat & Case report & 1 & TSH 0.03 vs. 73 & $\mathrm{n} / \mathrm{a}$ & 150 \\
\hline $\begin{array}{l}\text { Cation } \\
\text { exchange } \\
\text { resin }\end{array}$ & Case report & 1 & TSH 0.67 vs. 139 & $\begin{array}{l}\text { Yes, } \mathrm{T}_{4} \text { adsorbed to } \\
\text { sodium polystyrene } \\
\text { sulfonate }\end{array}$ & 151 \\
\hline
\end{tabular}

n/a, not applicable; TSH, thyrotropin.

$(99,100,169)$. As these three studies antedated the current FDA guidelines for the manufacture of $\mathrm{LT}_{4}$ products, they illustrate the concept of formulation differences, but are not directly applicable to the identifiable formulations currently available. Anecdotal reports of TSH changes associated with different $\mathrm{LT}_{4}$ products exist (170). Similar anecdotal reports of changes in serum TSH when a patient's $\mathrm{LT}_{4}$ is changed either from one brand name to another, or from a brand name to generic product, or from one generic to another have been gathered using an independent "Thyroid Pharmacovigilance" website (171). A point to be considered when evaluating these reports is that serum TSH may also fluctuate while a patient is maintained on a particular product, due to variable adherence and other factors. As mentioned in the discussion of recommendation 2a, a recent prospective, cross-over trial, conducted in the pediatric population, showed that similar doses of a branded product and a generic considered by the FDA to be interchangeable resulted in significantly different TSH values in children being treated for $\mathrm{CH}$ (107). This study is interesting, but may not be generalizable to the adult population.

Taken together, such evidence favors consistent use of the same identifiable formulation of $\mathrm{LT}_{4}$ to avoid altered ab- sorption potentially resulting in a different serum TSH. As mentioned previously, if a patient's $\mathrm{LT}_{4}$ product is switched, the endocrine societies recommend that the patient undergo repeat assessment of their thyroid status once steady state has been achieved (97). Altered TSH values associated with changes between $\mathrm{LT}_{4}$ products have only been documented with products no longer marketed in the same form, in anecdotal reports, and now, in a randomized study in children. Systematic studies of currently available $\mathrm{LT}_{4}$ products in patients over 18 years of age could lead to a stronger conclusion regarding the differential impact of their absorption on the TSH values achieved during therapy in adults.

\section{3e. What medications may alter a patient's levothyroxine requirement by affecting either metabolism or binding to transport proteins?}

\section{- RECOMMENDATION}

Initiation or discontinuation of estrogen and androgens should be followed by reassessment of serum thyrotropin at steady state, since such medications may alter the levothyroxine requirement. Serum thyrotropin should also 
be reassessed in patients who are started on agents such as tyrosine kinase inhibitors that affect thyroxine metabolism and thyroxine or triiodothyronine deiodination. Serum thyrotropin monitoring is also advisable when medications such as phenobarbital, phenytoin, carbamazepine, rifampin, and sertraline are started.

\section{Strong recommendation. Low-quality evidence.}

\section{Discussion of the clinical literature}

Many medications may necessitate an adjustment in $\mathrm{LT}_{4}$ dose by virtue of altering $\mathrm{T}_{4}$ metabolism or changing the concentration of thyroxine-binding globulin (TBG). $\mathrm{T}_{4}$ and $\mathrm{T}_{3}$ are primarily metabolized by deiodination, but are also metabolized by conjugation with glucuronates and sulfates in the liver. All studies cited are either case reports or nonrandomized prospective studies.

The major enzymes involved in conjugation are the mixed function oxidases and the uridine diphosphate-glucuronosyltransferases. Literature review revealed several relevant studies conducted in hypothyroid patients. Examples of drugs that have either been shown to increase hepatic metabolism of $\mathrm{T}_{4}$ and $\mathrm{T}_{3}$ via their induction of these enzymes, or are presumed or hypothesized to have this effect, include phenobarbital, phenytoin, carbamazepine, rifampin, sertraline, and possibly imatinib (172). The effect of phenobarbital to increase $T_{4}$ and $T_{3}$ metabolism has been documented in rodents (173) and humans $(174,175)$, but effects on thyroid function are more notable in rats (172). Often patients or healthy volunteers taking phenytoin remain euthyroid $(176,177)$. A case report describes the increased TSH seen in a patient prescribed phenytoin, while she maintained her $\mathrm{LT}_{4}$ dose (178). Other case reports also describe the development of hypothyroidism in patients treated with $\mathrm{LT}_{4}$, who were prescribed phenytoin $(179,180)$. Decreased $\mathrm{T}_{4}$ and $\mathrm{T}_{3}$ concentrations without an alteration in TSH were reported in 13 patients without pre-existing hypothyroidism (176) and nine hypothyroid patients (181) after initiation of carbamazepine therapy. A similar phenomenon (normal TSH and low $\mathrm{FT}_{4}$ ) has been attributed to a measurement artifact in the case of free thyroid hormone concentrations (182). Longer therapy with two months of carbamazepine caused a substantial increase in TSH in three of five hypothyroid children receiving $\mathrm{LT}_{4}$ (183). Case reports in a single athyreotic patient (184) and three patients with Hashimoto's hypothyroidism (185) document a substantially increased TSH and subsequent need for increased $\mathrm{LT}_{4}$ dose with introduction of rifampin. An increase in TSH associated with sertraline therapy was reported in 11 patients (186), although another study did not report such an increase (187). All of these studies highlight the need to be alert for the possibility of increased $\mathrm{LT}_{4}$ requirement in patients receiving drugs that can affect $\mathrm{LT}_{4}$ metabolism by conjugation.

The need for a substantially increased dose of $\mathrm{LT}_{4}$ has been reported in athyreotic patients prescribed tyrosine kinase inhibitors such as imatinib (188), motesanib (189), sorafenib (190), sunitinib, and vandetanib (191). Accelerated conversion of $\mathrm{T}_{3}$ to $\mathrm{rT}_{3}$ because of activation of D3 has been suggested to be one of the mechanisms by which tyrosine kinase inhibitors increase the $\mathrm{LT}_{4}$ requirement in hypothyroid patients $(190,191)$. The activity of other deiodinases may also be affected by these agents (191).

With respect to agents that alter D2 activity, a need for an increased dose of $\mathrm{LT}_{4}$ was reported with initiation of amiodar- one therapy in two patients (192), and this drug has been shown to inhibit D2 in rodents (193). The iodine content of amiodarone could also be responsible for worsening of hypothyroidism in patients with intact thyroid glands. Although there are reports in humans that propranolol and glucocorticoids also inhibit $\mathrm{T}_{4}$ to $\mathrm{T}_{3}$ conversion $(194,195)$, there do not seem to be data indicating that these agents increase the requirement for $\mathrm{LT}_{4}$ in hypothyroid patients. Growth hormone $(\mathrm{GH})$ replacement therapy in $\mathrm{GH}$-deficient subjects decreases serum $\mathrm{T}_{4}$ and $\mathrm{rT}_{3}$ levels, with a concomitant rise in serum $\mathrm{T}_{3}$ levels $(196,197)$. This suggests a stimulatory effect of $\mathrm{GH}$ and/or insulin-like growth factor 1 on the peripheral deiodination of $\mathrm{T}_{4}$. As a consequence, $\mathrm{LT}_{4}$ dose may need to be adjusted when growth hormone therapy is initiated. Although consumptive hypothyroidism has most usually been reported in children with hemangiomas, it has also rarely been reported as a cause of increased $\mathrm{LT}_{4}$ requirement in adults. Vascular tumors (198), fibroblastic tumors (199), and gastrointestinal stromal tumors (200) may each overexpress D3 and result in a need for an increased dose of $\mathrm{LT}_{4}$.

If drugs that increase the serum TBG concentrations, and thus increase total $\mathrm{T}_{4}$ levels, are started in hypothyroid patients receiving $\mathrm{LT}_{4}$, their TSH levels may rise above the reference range, thus signaling the need for a higher dose of $\mathrm{LT}_{4}$ to compensate and restore the steady state. Examples of drugs that increase serum TBG from insignificant to significant degrees, include estrogen (201), tamoxifen (202), raloxifene (203), clofibrate (204), opioids (205), mitotane (206), fluorouracil (207), and capecitabine (208). With the exception of estrogen, these drugs have either not been associated with an altered TSH (202-207), or were shown in case reports only (208). With respect to the classic case of estrogen treatment in postmenopausal women, such therapy was associated with an increase in mean TSH concentration from $0.9 \mathrm{mIU} / \mathrm{L}$ to approximately $3.2 \mathrm{mIU} / \mathrm{L} 12$ weeks after initiation of estrogen therapy in a prospective nonrandomized study (201). Rapid estrogen increase during in vitro fertilization is associated with increased TBG (209), and increased TSH values in treated hypothyroid women (210). Data addressing the importance of reversing such acute changes in TSH are lacking. Androgens have the opposite effect and decrease the serum TBG concentrations, thus necessitating a decrease in $\mathrm{LT}_{4}$ dose, as seen in an observational study of four patients (211).

\section{Levothyroxine Dosage}

\section{4a. What factors determine the levothyroxine dose required by a hypothyroid patient for reaching the appropriate serum TSH goal?}

\section{- RECOMMENDATION}

When deciding on a starting dose of levothyroxine, the patient's weight, lean body mass, pregnancy status, etiology of hypothyroidism, degree of thyrotropin elevation, age, and general clinical context, including the presence of cardiac disease, should all be considered. In addition, the serum thyrotropin goal appropriate for the clinical situation should also be considered.

\section{Strong recommendation. Moderate quality evidence.}

\section{Discussion of the clinical literature}

Many factors can affect the $\mathrm{LT}_{4}$ dose required to normalize a particular patient's TSH. Most studies examining this issue 
were retrospective chart reviews (47,212-224), but some studies were prospective (38,225-229). Much of these data are based on retrospective or cross-sectional studies, although the conclusions of these studies are generally consistent with each other.

There is consistent evidence that actual body weight, TSH goal (normal versus subnormal), ideal body weight, etiology of hypothyroidism, degree of serum TSH elevation, pregnancy, and age can influence dose requirement. Based on body weight, hypothyroid patients with minimal endogenous thyroid function require $\mathrm{LT}_{4}$ doses of $1.6-1.8 \mu \mathrm{g} / \mathrm{kg}$ of actual body weight $(38,47,212,213,215-217,226,227)$, although some studies estimate higher doses of $2.0-2.1 \mu \mathrm{g} / \mathrm{kg}$ for some patient groups $(212,213,226)$. Two studies have shown that ideal body weight is a better predictor of $\mathrm{LT}_{4}$ dose than actual body weight $(214,225)$, thus suggesting that $\mathrm{LT}_{4}$ dose requirement may depend on lean body mass. $\mathrm{LT}_{4}$ doses in thyroid cancer patients requiring TSH suppression are generally higher and on the order of $2.1-2.7 \mu \mathrm{g} / \mathrm{kg}(38,212,215)$.

The etiology of a patient's hypothyroidism affects their $\mathrm{LT}_{4}$ dose $(212,226)$, likely reflecting the amount of residual functional thyroid tissue. Patients who are athyreotic as a consequence of thyroidectomy generally require a higher $\mathrm{LT}_{4}$ dose than patients with Hashimoto's thyroiditis. Patients who have received radioiodine therapy for Graves' hyperthyroidism may have a variable need for $\mathrm{LT}_{4}$, depending on whether they have remaining functional autonomous thyroid tissue. Pretreatment serum TSH may also determine dose requirement (229). Indeed, the magnitude of the serum TSH elevation in patients newly diagnosed with hypothyroidism has been used to accurately predict a patient's $\mathrm{LT}_{4}$ requirement at the time that their serum TSH was first rendered normal on two successive occasions (229).

Women being treated for hypothyroidism typically require an increase in their $\mathrm{LT}_{4}$ dose early in the first trimester of pregnancy (228). The magnitude of the increase is greater in patients with little residual thyroid function (218). $\mathrm{LT}_{4}$ replacement doses tend to decrease with age when they are titrated based on serum TSH $(213,215,216,219,220)$. This occurs despite the previously mentioned decrease in $\mathrm{LT}_{4}$ absorption with advancing age (35), illustrating that the relationship between $\mathrm{LT}_{4}$ dose requirement and age is complex.

In contrast to consistency of the findings reported in the above studies, studies examining the effect of sex $(214,215$, $221)$, menopausal status $(214,215,221)$, and the presence of the type 2 deiodinase gene (DIO2) Thr92Ala polymorphism $(222,224)$ have produced inconsistent findings. Differences in dose requirement based on sex and menopausal status have been reported in three studies $(214,215,221)$. However, although all the studies found a lower dose requirement in postmenopausal women, different conclusions were reached about the dose requirement of men. One study suggested that sex differences were best demonstrated when dose requirement was based on ideal body weight or degree of overweight was included in the model (214). This study concluded that the effect of sex on $\mathrm{LT}_{4}$ dose requirement was not removed by accounting for sex differences in ideal body weight or degree of overweight. Another study, in contrast, suggested that differences in lean body mass accounted for sex differences (225).

Two different studies have examined the effect of deiodinase polymorphisms on the $\mathrm{LT}_{4}$ dose required to reach a target TSH concentration. One study showed that the DIO2Thr92Ala polymorphism predicted the need for a higher dose of $\mathrm{LT}_{4}$ in order to achieve near suppression of the serum TSH of thyroid cancer patients (224). Another larger study, in contrast, found no effect of this polymorphism on the $\mathrm{LT}_{4}$ dose needed to achieve TSH suppression in thyroid cancer patients or TSH normalization in patients with Hashimoto's hypothyroidism (222). Thus, the study by Torlontano et al. (224) suggests a blunting in the thyrotroph response associated with diminished $\mathrm{T}_{4}$ to $\mathrm{T}_{3}$ conversion, such that a higher dose of $\mathrm{LT}_{4}$ is needed to suppress TSH. However, this theory may not be supported by the fact that the $\mathrm{FT}_{4}$ and $\mathrm{FT}_{3}$ levels did not differ between the two genotypes being studied. There are no data regarding the impact of this polymorphism in nonpituitary brain tissues. However, the pituitary is highly enriched in D2, hence it could be more sensitive (compared to other tissues) to the effects of relative defects in the D2 enzymatic activity. Given the inconsistent results of these two studies $(222,224)$, further studies will be needed to determine if a patient's genetic composition truly determines their $\mathrm{LT}_{4}$ requirement (see discussion in section 13a).

With respect to the effect of age on $\mathrm{LT}_{4}$ requirement, analyses of age-related changes have not always incorporated adjustments for both the weight and sex of patients. In one study that adjusted for body weight only, older patients still required a lower $\mathrm{LT}_{4}$ dose (213). In another study that investigated females and males separately, the decreased $\mathrm{LT}_{4}$ requirement with age was only documented in men (220). Two other studies have suggested that declining lean body mass (219) or alterations in body composition and/or changes associated with menopause (214) accounted for the reduced $\mathrm{LT}_{4}$ requirement with age. Interestingly, sick patients older than 65 years, who are taking other medications in addition to $\mathrm{LT}_{4}$ for a variety of comorbidities, require lower weight-based doses of $\mathrm{LT}_{4}$ to normalize their serum TSH than do healthy controls of a similar age who are taking only $\mathrm{LT}_{4}(223)$.

\section{4b. What is the best approach to initiating and adjusting levothyroxine therapy?}

\section{- RECOMMENDATION}

Thyroid hormone therapy should be initiated as an initial full replacement or as partial replacement with gradual increments in the dose titrated upward using serum thyrotropin as the goal. Dose adjustments should be made when there are large changes in body weight, with aging, and with pregnancy, with thyrotropin assessment 4-6 weeks after any dosage change.

\section{Strong recommendation. Moderate quality of evidence.}

\section{Discussion of the clinical literature}

Several approaches to initiating $\mathrm{LT}_{4}$ therapy are acceptable. One approach is to base the starting dose on the serum $\mathrm{TSH}$ level, with full replacement doses (roughly $0.73 \mu \mathrm{g} / \mathrm{lb}$ or $1.6 \mu \mathrm{g} / \mathrm{kg}$ body weight) being required when the serum TSH is markedly elevated, and lower doses (e.g., 25-50 $\mu \mathrm{g}$ ) being required in milder degrees of hypothyroidism; for example, if the serum TSH is $\leq 10 \mathrm{mIU} / \mathrm{L}$ or the patient has $\mathrm{SCH}(3,229)$. Other factors to consider when initiating $\mathrm{LT}_{4}$ therapy include patient age (220) and underlying comorbidities (223), both of which tend to decrease the daily hormonal requirement. The 
daily $\mathrm{LT}_{4}$ dose is more dependent on lean body mass than total body weight (225), which explains why the elderly often require lower doses of $\mathrm{LT}_{4}$ (219). Medications being taken concurrently may also affect the dose required (230).

The full calculated daily $\mathrm{LT}_{4}$ dose (based on body weight) may be given initially to young and middle-aged patients who are otherwise healthy, but many experts recommend that elderly patients and those with cardiovascular disease "start low and go slow," for fear of precipitating cardiac events. However, one randomized trial showed that even elderly hypothyroid patients who are free of cardiovascular disease, as assessed by a dobutamine stress echocardiogram and bicycle ergometry at 12 and 24 weeks, may be safely started on the full replacement dose (227). It was also observed that thyroid functional parameters improved more rapidly in patients given the full dose rather than lower doses. There was no difference in the time it took for hypothyroid symptoms to resolve, although the initial assessment of symptoms did not take place until 12 weeks. This has led to the recommendation by some experts that the practice of starting $\mathrm{LT}_{4}$ therapy slowly in the elderly not be abandoned, since it appears that there is little to be gained in elderly patients by starting with a full dose, and there are potential risks unless it has been documented that the patient is free of cardiovascular disease (231). Patients with known coronary artery disease (CAD) should always be started on a low $\mathrm{LT}_{4}$ dose $(12.5-25 \mu \mathrm{g} / \mathrm{d})$, with gradual increases based on symptoms and serum TSH levels. Patients who have been rendered profoundly hypothyroid for radioiodine scanning and treatment for thyroid cancer or who became hypothyroid after treatment for hyperthyroidism would be an exception to the recommendation to "go slow" because they were recently either hyperthyroid or euthyroid and have experienced hypothyroidism of limited duration.

With the exception of secondary (central) hypothyroidism, rare instances of peripheral thyroid hormone resistance, and analytic interference with its measurement, the serum TSH is the parameter that is used to adjust the $\mathrm{LT}_{4}$ dose, with the target TSH typically being 0.5 to 3.5 or $4 \mathrm{mIU} / \mathrm{L}$. Dose adjustments are usually made 4-6 weeks after thyroid hormone is initiated, based on the half-life of $\mathrm{LT}_{4}$, which reaches steady-state levels by then, and serum TSH, which reaches its nadir at the same time. The target serum TSH may vary depending on patient age and underlying comorbidities. In general, $\mathrm{LT}_{4}$ dose adjustments of $12.5-25 \mu \mathrm{g} / \mathrm{d}$ are made, either up or down, depending on whether the serum TSH is high or low, respectively; the serum TSH is then repeated in 4-6 weeks, until the TSH target has been reached. Thereafter, serum TSH should be measured in 4-6 months and then yearly to assure stability. Changes in $\mathrm{LT}_{4}$ requirements occur with progression of thyroid failure (higher), aging (lower), weight loss (lower), and pregnancy (higher), and many other factors, such as concomitant medications, altered intestinal absorption, and medication adherence, to name a few. Interestingly, patients being prescribed lower doses of $\mathrm{LT}_{4}$ appear to have more stable TSH values over time than those taking higher doses (232).

\section{4c. What are the potential deleterious effects of excessive levothyroxine?}

\section{- RECOMMENDATION}

The deleterious health effects of iatrogenic thyrotoxicosis include atrial fibrillation and osteoporosis. Because of these effects we recommend avoiding thyroid hormone excess and subnormal serum thyrotropin values, particularly thyrotropin values below $0.1 \mathrm{mIU} / \mathrm{L}$, especially in older persons and postmenopausal women.

\section{Strong recommendation. Moderate quality evidence.}

\section{Discussion of the clinical literature}

Unfortunately, many patients treated with $\mathrm{LT}_{4}$ are overtreated, based on subnormal serum TSH levels. In one study of older individuals taking $\mathrm{LT}_{4}, 36 \%$ of patients over age 65 years had a subnormal serum TSH (233). Excess levels of thyroid hormones, especially levels that lead to serum TSH $<0.1 \mathrm{mIU} /$ $\mathrm{L}$, have been shown in many studies to be associated with adverse outcomes, especially related to the cardiovascular system and the skeleton in older persons or postmenopausal women. For example, in one study, patients older than age 65 with serum TSH levels $<0.1 \mathrm{mIU} / \mathrm{L}$, the majority of whom were taking $\mathrm{LT}_{4}$, had a threefold increase in the risk of atrial fibrillation over a 10-year observation period compared to euthyroid controls (234). The risk for low bone density and fractures is also elevated in postmenopausal (but not premenopausal) women taking $\mathrm{LT}_{4}(235)$, especially if the serum TSH levels are undetectable (236). The hazard ratios for inpatient admissions and deaths due to cardiovascular disease, dysrhythmias, and osteoporotic fractures were higher for those with suppressed TSH values in the same study of $\mathrm{LT}_{4}$-treated patients (236). Balancing the risks and benefits of subnormal TSH values in individuals with differentiated thyroid cancer will not be addressed in this document.

\section{4d. What are the potential deleterious effects of inadequate levothyroxine?}

\section{- RECOMMENDATION}

The adverse effects of thyroid hormone deficiency include detrimental effects on the serum lipid profile and progression of cardiovascular disease. We recommend that patients with overt hypothyroidism be treated with doses of levothyroxine that are adequate to normalize serum thyrotropin levels, in order to reduce or eliminate these undesirable effects.

\section{Strong recommendation. Moderate quality evidence.}

\section{Discussion of the clinical literature}

Just as $\mathrm{LT}_{4}$ therapy is frequently associated with overtreatment, undertreatment is common as well (233). Whether this is due to poor medication adherence or inadequate monitoring by the provider and failure to appropriately adjust the $\mathrm{LT}_{4}$ dose is not known. To the extent that the goal of treating hypothyroidism is to reverse the adverse effects of thyroid hormone deficiency on all body systems, inadequate therapy would be expected to be associated with the same comorbidities as untreated disease, especially dyslipidemia (81), atherosclerotic cardiovascular disease (237), and congestive heart failure (238), although likely to a less severe degree. Severe hypothyroidism is also a cause of reversible cardiomyopathy (239).

In mild (subclinical) hypothyroidism, however, it has been difficult to show that the mildly elevated serum TSH levels typical of this state are associated with adverse outcomes when 
left untreated, so that inadequate treatment would not necessarily be associated with morbidity. However, a recent report suggested that treatment of mild hypothyroidism was of benefit in reducing the risk of cardiac events in patients aged 40 70 years, providing some preliminary evidence in favor of normalizing the serum TSH in this age group (53). In this same study, however, no benefit of treatment was seen in persons over age 70 years, making it difficult to state that inadequate $\mathrm{LT}_{4}$ therapy of SCH has deleterious effects in older persons. Indeed, the target serum TSH in the elderly may be higher than in younger persons, in light of data showing that older persons have a shift to the right in the serum TSH distribution, making the concept of inadequate therapy even more problematic in this age group (see recommendation 6a).

The particular importance of avoiding undertreatment of hypothyroidism during pregnancy is discussed in section $6 \mathrm{~b}$.

\section{Levothyroxine and Other Nonhypothyroid Medical Conditions}

\section{5a. What is the appropriate management of perceived allergy to the constituents of levothyroxine or intolerance to levothyroxine?}

\section{- RECOMMENDATION}

Perceived allergy or intolerance to levothyroxine can be managed by changing the dose or product, including consideration of gel capsules, and possibly by treating concomitant iron-deficiency anemia. In selected cases, a consultation with an allergist may be appropriate.

Weak recommendation. Low-quality evidence.

\section{Discussion of the clinical literature}

The vast majority of patients taking $\mathrm{LT}_{4}$ tolerate the medication without adverse effects. Since $\mathrm{LT}_{4}$ is identical to the molecule produced by the body, it is difficult to understand how patients can be "allergic" to the medication, but occasional patients perceive adverse reactions from the medication, including headaches, palpitations, anxiety, and other nonspecific symptoms even when their thyroid hormone levels are still low and serum TSH levels are elevated. A reasonable approach in such instances would be to reduce the $\mathrm{LT}_{4}$ dose and advance it slowly. One report of symptoms with institution of $\mathrm{LT}_{4}$ therapy documented resolution of the symptoms when concomitant iron deficiency was corrected (240), highlighting that the correct attribution of the cause of symptoms is not always easy. Allergy to the dye in the tablet may rarely occur (241) and can be managed using $50 \mu \mathrm{g}$ tablets, which are colorant-free. A recent report of an allergy in the form of a rash that developed in a patient taking an $\mathrm{LT}_{4}$ preparation manufactured in Korea and containing tartrazine yellow no. 4 and red no. 3, was circumvented by treating the patient with a different levothyroxine product (242). Purported intolerance to other excipients in $\mathrm{LT}_{4}$ tablets (e.g., lactose, acacia, or even gluten) is anecdotal. However, some brands of $\mathrm{LT}_{4}$ contain lactose and/or acacia, and others do not, so trying an alternate product, including gel capsules, to see if allergic symptoms resolve is a reasonable strategy. If problems persist, a referral to an allergist may be helpful to rule out other allergens in food or in the home, reactions to which may have been attributed to the patient's thyroid medication.

\section{5b. How do co-existent medical conditions (e.g., atherosclerotic coronary artery disease) affect the management of levothyroxine replacement therapy?}

\section{- RECOMMENDATION}

We recommend considering patients' underlying medical conditions (such as atherosclerotic heart disease) in order to establish realistic treatment goals and avoid exacerbation of underlying comorbidities.

Weak recommendation. Low-quality evidence.

\section{Discussion of the clinical literature}

Renal and liver disease. There are no adjustments in $\mathrm{LT}_{4}$ dosing that are required in cases of cirrhosis or renal failure. Nephrotic syndrome, with its large urinary protein losses that include the thyroid hormone transport proteins TBG, transthyretine and albumin, can be a cause of increased $\mathrm{LT}_{4}$ requirements due to excessive urinary thyroid hormone losses (243).

Cardiac disease. Thyroid hormone therapy, with its inotropic and chronotropic effects on the heart, is a potential cause of angina in patients with severe CAD $(244,245)$. This is the reason for starting patients with low doses of $\mathrm{LT}_{4}$, and increasing the dose slowly, while monitoring for the development of angina or other cardiac symptoms such as tachyarrhythmias. With the current use of $\beta$-adrenergic blocking drugs, most patients with CAD can be fully treated with $\mathrm{LT}_{4}$ without difficulty. If patients are unable to tolerate the full $\mathrm{LT}_{4}$ dose required to normalize the serum $\mathrm{TSH}$, additional measures (medical or surgical) to treat the CAD would be indicated.

Gastrointestinal disease. See recommendation $3 \mathrm{c}$ for discussion of gastritis, celiac disease, and intestinal by-pass surgery

\section{5c. How do co-existent psychosocial, behavioral, and mental health conditions (such as addiction, somatization disorder, and depression) affect the management of levothyroxine therapy?}

\section{- RECOMMENDATION}

The treatment goals of hypothyroidism are the same for patients with psychosocial, behavioral, and mental health conditions, as for the general population. However, referral to a mental health professional should be considered if the severity of the symptoms is not sufficiently explained by the severity of the biochemically confirmed thyroid dysfunction or another medical condition, or if the mental health condition is impairing effective management of levothyroxine replacement therapy.

\section{Strong recommendation. Low-quality evidence.}

\section{Discussion of the clinical literature}

Underlying mental health problems, such as depression, personality disorders (e.g., borderline personality), and addictions, may complicate treatment of hypothyroidism and become frank barriers to informed consent and may impact perception of health state and adversely affect rational 
decision-making capacity. Patients in these categories should have a formal capacity assessment by a mental health expert (e.g., psychiatrist, clinical psychologist or social worker, licensed addiction therapist) to rule out underlying mental health conditions.

One mental health disorder that has been hypothesized to be overlooked in the context of hypothyroidism is somatization disorder, which warrants evaluation and possible treatment by a mental health professional. Somatization disorder involves a range of physiological sensations and complaints manifest in response to a complex psychological or abuse history (246249). It is not a factitious disorder or malingering. It has been hypothesized that patients with somatization disorders, who have been treated for hypothyroidism, may persistently complain of a range of symptoms associated with hypothyroidism despite normal laboratory testing (250). Such patients are typically driven to a range of multiple practitioners, who may do multiple work-ups, and even unnecessary procedures. Such patients are frequently at risk for a range of iatrogenic harms, such as risks from unnecessary surgeries. They may also pay large sums of money for nonstandard alternative therapies to deal with their physiologic complaints in an attempt to "prove" they are real (as to the patient, they are), and they may become belligerent and combative when told they are euthyroid. Patients with somatization disorders are frequently misdiagnosed and mismanaged and have complicated medical histories. Frequent misdiagnosis may occur because they seek out so many subspecialists. Somatization disorder is overwhelmingly diagnosed in females, with current hypotheses that it may be a disorder of affect regulation (251) or a complication or manifestation of a history of physical or sexual abuse (252). Recent data suggest that one in three women worldwide have been sexually or physically abused in their lifetimes (domesticviolencestatistics.org). Somatization disorder should be managed in conjunction with a mental health care provider to rule out other underlying psychiatric problems, including personality disorders.

In patients with persistent complaints of hypothyroidism as well as chronic pain and malaise, all organic causes should be ruled out, followed by referral to a mental health practitioner to screen for somatoform disorder. Patients suspected of somatoform disorders should be provided with sensitive discussion in which the referral is explained, in which trust is maintained. Patients should understand and appreciate that their symptoms are not factitious and are real and they may have causes that are rooted in psychological trauma, rather than an organic problem with physiologic causes.

\section{Levothyroxine Therapy in Specific Subpopulations}

\section{6a. How should levothyroxine therapy be managed in the elderly with hypothyroidism?}

\section{- RECOMMENDATION}

In general, levothyroxine should be initiated with low doses, and the dose titrated slowly based on serum thyrotropin measurements. It should be recognized that normal serum thyrotropin ranges are higher in older populations (such as those over 65 years), and that higher serum thyrotropin targets may be appropriate.

Strong recommendation. Moderate quality evidence.

\section{Discussion of the clinical literature}

In elderly persons (those older than 65-70 years) who are without known heart disease or without major risk factors for heart disease, $\mathrm{LT}_{4}$ therapy can be initiated at the full dose (227), although the method of starting with a low dose and increasing it slowly is still preferred by some experts (231). The final $\mathrm{LT}_{4}$ dose that normalizes the serum TSH is generally lower in the elderly compared to younger persons $(213,220)$, related to decreases in $\mathrm{T}_{4}$ turnover with age, in turn caused by decreases in lean body mass (253). However, other factors in the elderly, such as decreased thyroid hormone absorption; concomitant drug use, which is more common in the elderly; and other comorbidities may serve to negate the effects of decreased $\mathrm{T}_{4}$ metabolism, leading to an $\mathrm{LT}_{4}$ dose that is closer to what would be predicted in a younger person.

The elderly are more susceptible to the adverse effects of thyroid hormone excess, especially atrial fibrillation (234), and osteoporotic fractures $(235,236,254)$, so that careful titration of the $\mathrm{LT}_{4}$ dose to avoid iatrogenic thyrotoxicosis is essential in this population. Studies specifically examining methods for dose titration in the elderly could not be identified, thus making clinical judgment of paramount importance. In addition to the lower dose requirements related to $\mathrm{T}_{4}$ metabolism, the target serum TSH should likely be raised in older persons, especially the oldest old (patients $>80$ years), given data showing that serum TSH levels rise with age in normal individuals who are free of thyroid disease (255257). Indeed, the $97.5 \%$ confidence interval for serum TSH in healthy elderly persons is $7.5 \mathrm{mIU} / \mathrm{L}$ (255). There are observational data showing decreased mortality rates (258) and improved measures of well-being (259) in elderly persons with TSH levels that are above the traditional reference range (i.e., $0.5-4.5 \mathrm{mIU} / \mathrm{L}$ ) for the general population. Furthermore, there are also observational data showing that higher $\mathrm{FT}_{4}$ concentrations are associated with mortality in the elderly (257). On the other hand, some data suggest that that subclinical hypothyroidism may be associated with increased mortality, possibly limited to those with cardiac disease such as congestive heart failure (260).

No RCTs of $\mathrm{LT}_{4}$ treatment in elderly patients with hypothyroidism comparing different TSH target values are available. However, based on the current evidence it is reasonable to raise the target serum TSH to 4-6 mIU/L in persons greater than age 70-80 years. Given that many individuals taking $\mathrm{LT}_{4}$ are either undertreated or overtreated $(49,233)$, and that, in addition, those aged 85 years and older are more frequently started on $\mathrm{LT}_{4}$ than younger age groups (261), the elderly, who comprise an increasing proportion of the U.S. population, should be targeted for particularly careful monitoring of therapy. If maintaining a regular daily schedule of $\mathrm{LT}_{4}$ therapy is problematic in an elderly individual, consideration could be given to a guardian or visiting nurse giving all the $\mathrm{LT}_{4}$ pills once weekly (or half the pills twice weekly), if appropriate.

$\mathrm{LT}_{4}$ management in the case of elderly who are incapacitated and require guardianship is beyond the scope of this document. However, elderly patients who are no longer competent/capacitated may be overlooked with respect to effective treatment of hypothyroidism and should be considered to be a vulnerable group. 


\section{6b. How should levothyroxine therapy be managed in pregnant women with hypothyroidism?}

\section{- RECOMMENDATION}

Women with overt hypothyroidism should receive levothyroxine replacement therapy with the dose titrated to achieve a thyrotropin concentration within the trimesterspecific reference range. Serial serum thyrotropin levels should be assessed every 4 weeks during the first half of pregnancy in order to adjust levothyroxine dosing to maintain thyrotropin within the trimester specific range. Serum thyrotropin should also be reassessed during the second half of pregnancy. For women already taking levothyroxine, two additional doses per week of the current levothyroxine dose, given as one extra dose twice weekly with several days separation, may be started as soon as pregnancy is confirmed.

\section{Strong recommendation. Moderate quality evidence.}

\section{Discussion of the clinical literature}

The treatment of hypothyroidism during pregnancy must be considered within the context of trimester-specific alterations in thyroid physiology as well as the etiology of the thyroid disease. The TSH range for each trimester should be defined within the medical system in which care is being provided, with a generalized range as follows: $0.1-2.5 \mathrm{mIU} / \mathrm{L}$ for the first trimester, $0.2-3.0 \mathrm{mIU} / \mathrm{L}$ for the second trimester, and $0.3-3.0 \mathrm{mIU} / \mathrm{L}$ for the third trimester, as outlined in the ATA guidelines for the management of thyroid disease during pregnancy (1). Several studies, including the Generation $\mathrm{R}$ study by Medici et al. (262), have reported a higher TSH reference range during pregnancy, suggesting that ethnicity-based polymorphisms and dietary factors may play an important role in the thyroid axis response to pregnancy (262-266). The use of $\mathrm{FT}_{4}$ immunoassays to further define the thyroid status during pregnancy is complicated by interferences associated with physiological changes during pregnancy. These changes include increases in TBG and nonesterified fatty acids, as well as lower concentrations of albumin. Liquid chromatography-tandem mass spectrometry (LC/MS/MS) assays eliminate some of these confounding factors (267). However, these assays are time consuming and more expensive. The increasing availability of LC/MS/MS assays may increase their acceptance and decrease their cost. LC/MS/MS appears to be associated with improved accuracy and reliability of $\mathrm{FT}_{4}$ measurements (268) and is the method endorsed by the ATA guidelines for the diagnosis and management of thyroid disease during pregnancy (1).

Overt hypothyroidism $(\mathrm{OH})$ is associated with an increased rate of maternal complications, including decreased fertility, increased miscarriage or stillbirth, hypertension, and postpartum hemorrhage $(269,270)$. In addition, $\mathrm{OH}$ is also associated with increased risks of fetal/infant complications, including preterm delivery, low birth weight, and irreversible cognitive deficits (271-274). In women previously treated for hypothyroidism or found to have $\mathrm{OH}$ during pregnancy, $\mathrm{LT}_{4}$ replacement therapy should be continued or initiated if not previously started (1). Ideally, the $\mathrm{LT}_{4}$ dose should be adjusted to achieve a preconception TSH level below $2.5 \mathrm{mIU} / \mathrm{L}$ (1). For women taking $\mathrm{LT}_{4}$ prior to pregnancy, the initial adjustment in $\mathrm{LT}_{4}$ dose may be accomplished by increasing the current dose by two tablets per week (228). Thyroid function should be mon- itored every 4-6 weeks, at least for the first and second trimesters [generally the time when thyroid hormone requirements are changing (228)] in order to determine if and when additional adjustments in $\mathrm{LT}_{4}$ dose are needed. Thyroid function should also be reassessed during the third trimester. Up to $70 \%$ of women will require adjustments of $30 \%$ or more from the preconception dose $(228,269,275-277)$. Proper treatment of maternal hypothyroidism is associated with improvement in some maternal and neonatal/infant outcomes $(278,279)$. Although not ideal, the rare circumstance of late treatment with $\mathrm{LT}_{4}$ does not universally result in poor neurodevelopmental outcome. In a recent case report by Downing et al. (280), near-normal cognitive development was achieved for three children born to mothers who had untreated hypothyroidism during the first trimester when compared to unaffected siblings, when maternal thyroid function was normalized prior to the third trimester. Women typically return to their lower prepregnancy requirement for $\mathrm{LT}_{4}$ after delivery.

\section{6c. How should levothyroxine therapy be managed in infants and children with overt hypothyroidism?}

\section{- RECOMMENDATION, infants}

Levothyroxine replacement at a dose of $10-15 \mu \mathrm{g} / \mathrm{kg} / \mathrm{d}$ should be initiated once newborn screening is positive, pending the results of confirmatory testing. Higher doses may be required for infants with severe congenital hypothyroidism. The aim of therapy is to maintain the serum thyroxine in the mid- to upper half of the pediatric reference range and the serum thyrotropin in the mid- to lower half of the pediatric reference range. The target should be to normalize serum thyroxine approximately 2-4 weeks after initiation of therapy. Once the proper dose is identified, surveillance testing with a serum thyrotropin and thyroxine should be performed every 1 to 2 months during the first year of life with decreasing frequency as the child ages.

\section{Strong recommendation. High-quality evidence.}

\section{- RECOMMENDATION, children}

All children with overt hypothyroidism should receive levothyroxine replacement therapy to normalize their biochemical parameters and reverse their signs and symptoms of hypothyroidism.

Strong recommendation. High-quality evidence.

\section{$6 d$. How should levothyroxine therapy be managed in children with subclinical hypothyroidism?}

\section{- RECOMMENDATION}

In children with subclinical hypothyroidism, due to the low risks of levothyroxine replacement therapy, many clinicians still consider it reasonable to initiate treatment to avoid any potential risk of negative impact on growth and development. Treatment is generally not recommended when the thyrotropin is $5-10 \mathrm{mIU} / \mathrm{L}$. For patients with subclinical hypothyroidism and thyrotropin $>10 \mathrm{mIU} / \mathrm{L}$ with signs and symptoms consistent with primary thyroid disease and/or risk factors associated with progression, levothyroxine replacement may be reasonable.

Weak recommendation. Low-quality evidence. 


\section{Discussion of the clinical literature}

The management of hypothyroidism in children is similar to adults; however, there are unique differences based on the requirement of normal thyroid function for neurocognitive development as well as growth and development. A reflection of the unique differences between child and adult physiology is the increased weight-based requirements for thyroid hormone replacement in children and adolescents compared to adults. As the child advances through the pediatric age into adulthood thyroid hormone replacement doses decrease, with newborns typically requiring $10 \mu \mathrm{g} / \mathrm{kg} / \mathrm{d}$, 1-year-old children $4-6 \mu \mathrm{g} / \mathrm{kg} / \mathrm{d}$, adolescents $2-4 \mu \mathrm{g} / \mathrm{kg} / \mathrm{d}$, with transition to the average adult dose of $1.6 \mu \mathrm{g} / \mathrm{kg} / \mathrm{d}$ once endocrine maturation is complete $(215,281)$.

Thyroid dysgenesis is the most common etiology of $\mathrm{CH}$, affecting approximately 1:2000 to 1:4000 newborns (282). While there is no consensus on the optimal starting dose of $\mathrm{LT}_{4}$ (283-286), standard or low-dose therapy is defined by a range of $5-10 \mu \mathrm{g} / \mathrm{kg} / \mathrm{d}$, while high-dose regimens are defined as a range of $10-15 \mu \mathrm{g} / \mathrm{kg} / \mathrm{d}(287,288)$. A systematic review of the literature summarizing data from 14 cohort studies with a total of 1321 patients reported no association between the starting dose of $\mathrm{LT}_{4}$ therapy and the standardized mean IQ or developmental quotient scores measured between 1 and 14 years of age (288). However, there are conflicting data, with several reports showing higher full-scale IQ scores associated with higher starting doses $(283,286)$. While the controversy remains, there is reasonable agreement that the severity of the $\mathrm{CH}$ at the time of diagnosis (the maximum TSH level) and the length of time to achieve normal thyroid function (TSH and $\mathrm{T}_{4}$ ) may ultimately have a greater impact on neurocognitive outcome than the initial starting dose $(283,286,289)$. In addition to the degree of TSH elevation, a distal femur plain radiograph may also help determine the severity of the hypothyroidism with a bony nucleus diameter $<3 \mathrm{~mm}$ in a term infant being associated with severe $\mathrm{CH}$ and decreased psychomotor development in the first year of life (290).

Optimal outcome appears to be achieved when thyroid hormone levels are normalized within 2 weeks of initiating therapy. Starting doses of $10-15 \mu \mathrm{g} / \mathrm{kg} / \mathrm{d}$ have been reported to normalize $\mathrm{T}_{4}$ levels within $2-3$ weeks, while doses $<8 \mu \mathrm{g} /$ $\mathrm{kg} / \mathrm{d}$ result in normalization within 6-8 weeks (288). Tailoring the dose based on the severity of initial TSH and $\mathrm{T}_{4}$ deficit may be the most reasonable approach. Further study of appropriate $\mathrm{LT}_{4}$ dosing in patients with $\mathrm{CH}$ treated with $\mathrm{LT}_{4}$ is certainly indicated. The addition of $\mathrm{LT}_{3}$ to $\mathrm{LT}_{4}$ has not been adequately studied to determine risk or benefit, but in a subgroup of patients with persistent elevations in TSH despite $\mathrm{T}_{4}$ levels in the mid to upper part of the reference range, the addition of $\mathrm{LT}_{3}$ may result in normalization of TSH (291). Within the United States, there are no liquid formulations and there are no compounding recipes that produce a stable concentration of $\mathrm{LT}_{4}$ in suspension. Efforts to create suspensions should be avoided and are associated with marked extremes in dosing. To improve the likelihood of compliance, $\mathrm{LT}_{4}$ should be administered during the same time of day, crushed and mixed with water, non-soy formula, or breast milk and administered via a spoon. Older children may chew or swallow the pill. Soy, iron, calcium, and infant colic drops (simethicone) can decrease the absorption of $\mathrm{LT}_{4}$ and concurrent ingestion should be avoided $(292,293)$.

The aim of treatment is to keep the $\mathrm{T}_{4}$ level in the mid to upper half of the reference range and the TSH in the mid to the lower half of the reference range, optimally between 0.5 and $2.0 \mathrm{mIU} / \mathrm{L}$ (287). Once the proper dose is identified, based on normalization of $\mathrm{TSH}$ and $\mathrm{T}_{4}$, surveillance testing with a TSH and $\mathrm{T}_{4}$ should be performed every 1-2 months during the first year of life with decreasing frequency as the child ages (287). Linear growth and development should be followed closely in conjunction with regularly scheduled, well-child health visits as recommended by the American Academy of Pediatrics. For patients with severe $\mathrm{CH}$, monthly surveillance during the first 6-12 months may be indicated (294). For patients with mild $\mathrm{CH}$ and no change in the $\mathrm{LT}_{4}$ dose over the first 3 years of life, a trial off $\mathrm{LT}_{4}$ therapy is reasonable in an effort to determine if the patient has transient or permanent $\mathrm{CH}$ (287). Permanent $\mathrm{CH}$ is established if the TSH rises and the $\mathrm{T}_{4}$ decreases on repeat testing 4-6 weeks after stopping $\mathrm{LT}_{4}$ therapy. For further information on the evaluation and management of $\mathrm{CH}$, please refer to the recent joint consensus statement from the European Society for Pediatric Endocrinology (295).

Autoimmune thyroiditis is the most common cause of acquired hypothyroidism, is more common in females compared to males, and the incidence increases during adolescence (296). Linear growth failure and concomitant bone age delay are the most common clinical signs of untreated acquired $\mathrm{OH}$. Uncommonly, children may present with severe hypothyroidism, with TSH values > $1000 \mathrm{mIU} / \mathrm{L}$ (297). The most likely etiology is late-onset $\mathrm{CH}$ or delayed diagnosis of autoimmune hypothyroidism. Several approaches to care have been attempted without much impact on improving compromised adult height, a common outcome. These approaches include initiation of small doses of $\mathrm{LT}_{4}$ with slowly advancing dose or the addition of gonadotropin-releasing hormone agonist therapy as soon as signs or puberty are witnessed or if the pace of pubertal advancement appears rapid (297,298). Reduction in pretreatment weight gain should not be presumed with the initiation of $\mathrm{LT}_{4}$ replacement therapy (299). $\mathrm{LT}_{4}$ replacement therapy is the treatment of choice for patients with $\mathrm{OH}$ with the dose adjusted for weight or body surface area (281). The $\mathrm{LT}_{4}$ dose for patients $1-3$ years of age is $4-6 \mu \mathrm{g} / \mathrm{kg} / \mathrm{d}$, for patients $3-10$ years the $\mathrm{LT}_{4}$ dose is 3-5 $\mu \mathrm{g} / \mathrm{kg} / \mathrm{d}$, and for patients 10-16 years the $\mathrm{LT}_{4}$ dose is $2-4 \mu \mathrm{g} / \mathrm{kg} / \mathrm{d}(281)$. $\mathrm{LT}_{4}$ may also be dosed based on body surface area calculated at $100 \mu \mathrm{g} / \mathrm{m}^{2} / \mathrm{d}$. The method of administration and the target values for $\mathrm{TSH}$ and $\mathrm{T}_{4}$ are similar to that previously described for neonatal $\mathrm{LT}_{4}$ replacement dosing.

$\mathrm{SCH}$ is similarly defined with a similar lack of consensus on if and/or when to initiate treatment (300-302). The majority of pediatric patients with $\mathrm{SCH}$ will not progress to $\mathrm{OH}$, and there does not appear to be a significant risk associated with not treating (303). In a large, retrospective study of 121,052 pediatric patients 6 months to 16 years of age, $73.6 \%$ of subjects with a TSH $>5.5$ to $\leq 10 \mathrm{mIU} / \mathrm{L}$ normalized their TSH over 5 years of follow-up. In subjects with a TSH $>10 \mathrm{mIU} / \mathrm{L}, 40 \%$ had normalization of their TSH values, 33.1\% had reduction of their TSH values, and only $25 \%$ had maintenance or increase of their TSH values (304).

The presence of anti-thyroid antibodies may help identifying a subpopulation at increased risk of progression to $\mathrm{OH}$. In a study following 382 patients, including 323 pediatric patients with Hashimoto's thyroiditis and 59 patients 
with isolated hyperthyrotropinemia, the presence of thyroid peroxidase antibodies (TPOAb) increased the risk of developing $\mathrm{OH}$ by 3.4-fold. However, after 3 years of follow-up, $78 \%$ of subjects with Hashimoto's thyroiditis and $86 \%$ of patients with isolated hyperthyrotropinemia did not develop $\mathrm{OH}(303)$.

In addition to a lack of evidence showing an increased risk of progression, there are no data showing short-term or longterm negative consequences associated with untreated $\mathrm{SCH}$ in the pediatric population, including no adverse effect on linear growth and no increase in cardiovascular risk, behavioral disorders, or problems with cognition (301,305-309). Normalization of TSH values in the range often seen in $\mathrm{SCH}$ ( $>5.5$ to $\leq 10 \mathrm{mIU} / \mathrm{L}$ ) may also occur in association with weight loss in obese pediatric patients, suggesting that the alterations in the thyroid axis are a consequence rather than a cause of the obesity (310).

In several subgroups of patients, however, the potential benefit of $\mathrm{LT}_{4}$ replacement therapy may be more significant and measurable, so the decision about whether to initiate $\mathrm{LT}_{4}$ replacement should be based not only on the level of TSH but also on the clinical scenario in which the test was ordered as well as the age and risk of progression. For patients with $\mathrm{SCH}$ and concern over linear growth, the presence of a goiter on exam or evidence of autoimmune disease (including TPOAb positivity and/or an associated autoimmune disorder such as celiac disease or diabetes), initiation of $\mathrm{LT}_{4}$ should be strongly considered secondary to potential benefit and an increased risk of progression (309). In addition, although there are no prospective data to show clear benefit from $\mathrm{LT}_{4}$ replacement, patients with $\mathrm{SCH}$ and an increased total cholesterol with elevated LDL fraction may also benefit from initiation of therapy $(310,311)$. Lastly, $\mathrm{LT}_{4}$ should also be considered in pediatric patients with a history of exposure to radiation for the treatment of a benign condition or nonthyroid malignancy due to an increased risk for progression from $\mathrm{SCH}$ to $\mathrm{OH}$, as well as evidence suggesting a lower risk of thyroid nodule formation with $\mathrm{LT}_{4}$ treatment (312). In a study examining 426 subjects previously treated for benign conditions with radiation, $14.2 \%$ of patients treated with $\mathrm{LT}_{4}$ developed recurrent nodules compared to $34.2 \%$ of 198 subjects that did not receive $\mathrm{LT}_{4}$ therapy (313).

\section{6e. How should levothyroxine therapy be managed in individuals who have elevated serum thyrotropin values due to nonadherence?}

\section{- RECOMMENDATION}

If prescription of daily levothyroxine is not successful in maintaining a normal serum thyrotropin, weekly oral administration of the full week's dose of levothyroxine should be considered in individuals in whom adherence cannot otherwise be sustained.

\section{Weak recommendation. Low-quality evidence.}

\section{Discussion of the clinical literature}

If patients maintain the same $\mathrm{LT}_{4}$ dose, their serum $\mathrm{TSH}$ levels should remain within a fairly narrow range $(37,314$ 316). If patients experience unexpected fluctuations in their serum TSH, or persistently elevated TSH concentrations despite the prescription of large doses of $\mathrm{LT}_{4}$, factors affecting $\mathrm{LT}_{4}$ formulation, absorption, and metabolism can be investigated as potential culprits. Loss of potency due to use of $\mathrm{LT}_{4}$ that is beyond its expiry date or use of pills that have deteriorated due to environmental causes such as excessive heat or moisture are other considerations. With respect to excessive heat, tablets may be heated during transport (particularly if shipped from a remote site) or may be stored in the heat (as may occur in patients whose jobs involve lengthy periods of travel in automobiles). If, however, such factors do not appear to be responsible, variable adherence or nonadherence to $\mathrm{LT}_{4}$ therapy should be considered (317). There are various reports in the literature in which patients with high TSH values while being prescribed $\mathrm{LT}_{4}$ were shown to be able to absorb $\mathrm{LT}_{4}$ normally (317-322) and based upon this documentation were thought to have poor adherence. Absorption testing can be conducted by administering a specific oral dose of $\mathrm{LT}_{4}$ under supervised conditions, measuring $\mathrm{T}_{4}$ concentrations at specific time points thereafter, and comparing the observed and predicted Cmax and AUC. A Cmax and AUC that is significantly less than the expected values would support impaired absorption. The principle is similar to that using for bioequivalence testing (see section 2a). Doses of $\mathrm{LT}_{4}$ of $600 \mu \mathrm{g}$ to $2 \mathrm{mg}$ have been employed in absorption testing (317-322).

If a combination of a high TSH and a normal or high $\mathrm{T}_{4}$ concentration is documented by laboratory testing, this pattern could be consistent with the syndrome of thyroid hormone resistance, a TSH-secreting pituitary adenoma, a macro-TSH phenomenon in which TSH complexes with an immunoglobulin and cannot be filtered by the kidney (323), or recent resumption of $\mathrm{LT}_{4}$ therapy (324). One survey of patients prescribed $\mathrm{LT}_{4}$ found a self-reported nonadherence rate of $22 \%$ (325).

Adherence may be made difficult for those taking multiple other medications including those that require specific administration conditions (e.g., bisphosphonates). Since $\mathrm{LT}_{4}$ is generally a lifelong medication, it is important for patients to identify a medication schedule that facilitates adherence. In addition, patients can be educated about what approach to take if they forget a dose. Given the long halflife of $\mathrm{LT}_{4}$, patients can safely be advised to make up any omitted doses. A weekly pill box may be helpful for identifying any omitted doses. Patients may also be taking medications that impair $\mathrm{LT}_{4}$ absorption. Variable absorption is clearly of less concern than omitted doses, so it may be necessary to compromise the goal of unimpaired absorption in order to facilitate adherence. There has been speculation that some cases of failure to adhere to a prescribed $\mathrm{LT}_{4}$ regimen may be linked to a psychiatric disorder manifest as a desire to remain a patient (326). Other psychosocial causes for noncompliance may include barriers to accessing medication, difficulties with insurance coverage, literacy issues, and lack of understanding regarding the benefits of taking $\mathrm{LT}_{4}$ as a medication.

Thyroid hormone concentrations in hypothyroid patients receiving large weekly doses of $\mathrm{LT}_{4}$ have been studied in the past, prior to routine documentation of TSH values $(327,328)$. Normalization of serum TSH in patients suspected of being nonadherent to their therapy, with weekly or twice weekly oral therapy has been reported in case reports $(319,326,329)$ and a study of a group of 23 patients (322). Literature review revealed four relevant controlled studies (330-332). 
Intuitively, patient education regarding the benefits of euthyroidism and the risks of iatrogenic thyroid disease would seem to a logical approach to reducing nonadherence. However, a randomized study providing education in the form of booklets mailed to patients' homes compared with usual care did not affect serum TSH, which was used as a surrogate marker of adherence (330). A randomized crossover trial of once weekly oral administration of 7 times the usual daily $\mathrm{LT}_{4}$ dose versus daily dosage administration showed that weekly therapy was associated with supratherapeutic concentrations of $\mathrm{T}_{4}$ for about 24 hours, although $\mathrm{T}_{3}$ levels remained within the reference range (331). Mean serum cholesterol was higher during weekly therapy, but other markers of $\mathrm{LT}_{4}$ action such as SHBG, osteocalcin, heart rate, and left ventricular ejection time did not differ between regimens over the 6-week duration of the trial. Patient symptoms and well-being also did not differ. A second similar cross-over trial of 6 weeks' duration showed greater increases in $\mathrm{T}_{4}$ after the weekly dose compared with the daily dose, with no associated symptoms or echocardiographic manifestations of hyperthyroidism (333). A small cross-over trial of daily oral therapy versus twice weekly oral therapy showed no significant differences in trough and peak $\mathrm{T}_{4}, \mathrm{~T}_{3}$, and TSH concentrations and no differences in systolic time intervals over a 1-month duration of therapy (332).

Parenteral $\mathrm{LT}_{4}$ therapy does not appear to have been rigorously studied. A case report in a patient with uncharacterized malabsorption, rather than noncompliance, has been described (334). Mathematical modeling of twice weekly or weekly administration of intramuscular $\mathrm{LT}_{4}$ showed some fluctuations of $\mathrm{T}_{4}$ levels, but with these levels remaining in the reference range (335).

In conclusion, if efforts to encourage regular daily consumption of $\mathrm{LT}_{4}$ are unsuccessful, options include observed therapy (329) or reduction of the frequency of $\mathrm{LT}_{4}$ ingestion to twice weekly (332) or weekly (331), with a starting dose equal to the weight-adjusted dose one would prescribe in a daily administration regimen (i.e., 7 times the daily dose). However, the three cross-over trials of such oral therapy were not long term, and none were conducted in a patient group previously reported as manifesting nonadherence (331-333). No differences in clinical or end organ parameters of thyroid hormone action, other than higher serum cholesterol with weekly therapy, were reported in these short-term trials. Parenteral administration of $\mathrm{LT}_{4}$ is also possible $(334,335)$, although no trials of such therapy were identified.

\section{Levothyroxine and Triiodothyronine Concentrations}

\section{7a. Are variations in serum triiodothyronine concentrations within the reference range of physiologic or clinical significance? In addition, are mildly low serum triiodothyronine concentrations of clinical significance?}

\section{- Summary statement}

The significance of perturbations in serum triiodothyronine concentrations within the reference range or of mildly low serum triiodothyronine concentrations is unknown.

\section{Mechanistic background}

Animal studies suggest that the thyroid axis has multiple homeostatic mechanisms that defend plasma $T_{3}$. Rodents subjected to iodine deficiency maintain their serum $T_{3}$ levels (336,337). However, when severe iodine deficiency is accompanied by a fall in serum $T_{3}$, one of the tissues that is unable to sustain normal $T_{3}$ concentrations is the brain (336). Interestingly, other rodent studies do not show deficient $T_{3}$ levels in the brain with $\mathrm{LT}_{4}$ monotherapy, although deficiency is seen in other tissues (338). Strikingly, animals with genetic inactivation of D1, D2, or both have serum $T_{3}$ levels within the normal range, though brain $T_{3}$ levels are low (339-341). Even if the D2 inactivation is restricted to the TSH-producing cells of the pituitary gland, there is resetting of the hypothalamic thyrotropin-releasing hormone (TRH) production and TSH biological activity in order to preserve serum $T_{3}$ (342). These observations and the finding that other thyroid parameters such as $T_{4}$ and TSH are deranged in these animals support the concept that the maintenance of circulating levels of $T_{3}$ is of primary importance in the thyroid axis homeostasis.

Plasma $T_{3}$ is the main, and perhaps the only, source of $T_{3}$ for tissues that lack D2, such as the liver (23). Even in D2expressing tissues, approximately $50 \%$ of the intracellular $\mathrm{T}_{3}$ is estimated to originate from the plasma based on rodent studies (23). Thus, if plasma $T_{3}$ was to fall to low levels, thyroid hormone signaling would be expected to decrease systemically. The minimum sustained decrease in plasma $T_{3}$ that results in biologically important effects remains to be determined. A general caveat for this discussion is that variation in $T_{3}$ assay performance represents one methodological limitation affecting attempts to extract data regarding $T_{3}$ concentrations from the existing literature. Another caveat of applying rodent studies to human physiology is that the $T_{4}: T_{3}$ ratio in rodents is skewed towards $T_{3}$, with a ratio of about 6:1 compared with 14:1 in humans, as pointed out by the authors in their study of plasma and tissue levels of $T_{4}$ and $T_{3}$ in thyroidectomized rats (338).

\section{Discussion of the clinical literature}

The interest in the use of combination therapies (see recommendation 13) or $\mathrm{T}_{3}$ monotherapy (see recommendation 14) implies that $T_{3}$ levels are important. This concept would be a shift in traditional thinking. According to the current model, normalizing serum TSH is generally considered the target of therapy, and serum $\mathrm{T}_{3}$ is typically not measured or monitored (343). Direct evidence addressing the question of whether small decrements in plasma $\mathrm{T}_{3}$ have clinically important sequelae is lacking. A critical question, therefore, is whether changes in $\mathrm{T}_{3}$ concentrations are important and more specifically whether decrements in $\mathrm{T}_{3}$ that remain within the reference range are actually of any clinical significance.

Some data in humans suggest that maintenance of serum $\mathrm{T}_{3}$ concentrations is of physiologic importance. It is well known that in mild primary hypothyroidism, serum $\mathrm{T}_{3}$ is kept normal in the face of a low serum $\mathrm{T}_{4}$ and mildly elevated TSH (4). In individuals living in areas of iodine deficiency, serum $\mathrm{T}_{3}$ levels are normal even when $\mathrm{T}_{4}$ and TSH are altered (344). In one study of 15 athyreotic patients taking $\mathrm{LT}_{4}$, a progressive $30 \%$ fall in $\mathrm{T}_{3}$ induced by PTU therapy over 8 days led to a doubling of TSH, even though $\mathrm{T}_{4}$ was constant (18). Given the wide interindividual variability of the serum $T_{3}$, a 
$30 \%$ decrease could occur with the actual $\mathrm{T}_{3}$ value nevertheless remaining in the normal reference range.

Serum $\mathrm{T}_{3}$ (345) and $\mathrm{FT}_{3}$ (346) levels vary in a circadian fashion in healthy adult subjects, with estimates of the delta ranging from $14 \%$ to $35 \%$. These changes likely follow the well-known circadian variation in TSH. Currently used therapies do not replicate the circadian rhythm of $\mathrm{T}_{3}$ or $\mathrm{FT}_{3}$. For example, patients taking $\mathrm{LT}_{4}$ monotherapy exhibit minimal variations in serum $\mathrm{T}_{3}$ (347). It is not known whether replication of this circadian rhythm is of biologic importance, or whether mirroring this rhythm affects the ability to reverse hypothyroidism. In contrast to the steady levels of $\mathrm{T}_{3}$ achieved with $\mathrm{LT}_{4}$ monotherapy, unless given multiple times daily, $\mathrm{LT}_{3}$ monotherapy is characterized by peaks and troughs in $\mathrm{T}_{3}$ concentration (348). The serum concentration of $\mathrm{T}_{3}$ following its oral administration can be predicted based on a two-compartment gut model (349). The peaks and troughs may not impact the genomic effects of $\mathrm{T}_{3}$, but may be important for nongenomic effects.

Study results are mixed as to whether $\mathrm{T}_{3}$ levels mirror other parameters that are generally thought to reflect thyroid status. Some studies show that $\mathrm{T}_{3}$ is unaltered when other thyroid-related parameters change. For example, in one study increases in $\mathrm{LT}_{4}$ dose that were reflected by increases in resting energy expenditure were not accompanied by significant changes in serum $\mathrm{T}_{3}$ concentration (51). In other studies, at least some thyroid-related parameters change with changes in $\mathrm{T}_{3}$. With respect to the diagnosis of hypothyroidism, total cholesterol and SHBG correlated well with $\mathrm{T}_{3}$, but had no correlation with TSH levels (350). In one study in which increasing $\mathrm{LT}_{4}$ dose also increased serum $\mathrm{T}_{3}$, there were accompanying changes in cholesterol and SHBG, but no changes in well-being (50). In another study of $\mathrm{LT}_{3}$ monotherapy, raising $\mathrm{T}_{3}$ levels to the higher end of the reference range was accompanied by weight loss, decreased cholesterol, and increased SHBG (347). In a study of hypothyroid patients, those with low $\mathrm{T}_{3}$ levels had higher scores of hypothyroidism than those with normal $\mathrm{T}_{3}$ levels (58). However, the latter group also had lower TSH concentrations. Despite this, the hypothyroidism score did not correlate with TSH. There are some cases in which $\mathrm{T}_{3}$ seems to be necessary for normalizing TSH. For example, there are case reports of children whose TSH values could only be normalized with a combination of both $\mathrm{LT}_{4}$ and $\mathrm{LT}_{3}$ (291). Concomitant $\mathrm{LT}_{4}$ and PTU therapy that resulted in normal serum $\mathrm{T}_{4}$ levels, but low serum $\mathrm{T}_{3}$ was associated with an elevated serum TSH (18).

A critical issue related to the importance of serum $\mathrm{T}_{3}$ levels is the performance of assays used to measure $\mathrm{T}_{3}$ and $\mathrm{FT}_{3}$. The disagreement between immunoassays and the more specific LC/MS/MS methodology is particularly noticeable at the lower end of the assay range, in the area where documentation of $\mathrm{T}_{3}$ concentrations would be most important if these levels were suspected to be deficient (351). LC/MS/MS assays have theoretical advantages and may be the assay method of choice in the future (352).

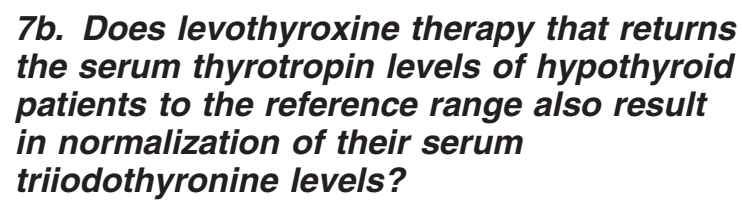

\section{- Summary statement}

Patients with hypothyroidism treated with levothyroxine to achieve normal serum TSH values may have serum triiodothyronine concentrations that are at the lower end of the reference range, or even below the reference range. The clinical significance of this is unknown.

\section{Discussion of the clinical literature}

$\mathrm{LT}_{4}$ is an effective, convenient, and cost-effective treatment for hypothyroidism (see recommendation 1a). One of the underlying concerns expressed about $\mathrm{LT}_{4}$ as a treatment for hypothyroidism in general is whether physiologic concentrations of $\mathrm{T}_{3}$ are provided by such therapy in patients who only retain minimal endogenous thyroid function. The implication is that inadequate $\mathrm{T}_{3}$ levels in either the circulation or in tissues may be responsible for failure to reverse symptoms of hypothyroidism.

There are at least eight studies $(38-43,353,354)$ examining the issue of serum $\mathrm{T}_{3}$ levels in humans being treated with $\mathrm{LT}_{4}$, although only two were prospective $(38,39)$. Two studies used subjects as their own controls $(39,42)$, five used matched controls $(38,40,41,43)$, and two used comparison to the laboratory reference range only $(353,354)$. A small prospective study of athyreotic patients with each patient serving as their own control showed maintenance of $\mathrm{T}_{3}$ concentrations within the group as a whole, although a higher $\mathrm{FT}_{4} / \mathrm{T}_{3}$ ratio was necessary to achieve this (39). A minority of individuals within the group was noted to have lower serum $\mathrm{T}_{3}$ levels while talking $\mathrm{LT}_{4}$. An additional prospective study also showed a comparable free $\mathrm{T}_{3}$ index in patients treated with $\mathrm{LT}_{4}$ (38). Further cross-sectional studies suggested maintenance of $\mathrm{T}_{3}$ concentrations in treated patients, but the reference range was the sole comparator $(353,354)$. However, three cross-sectional studies comparing $\mathrm{LT}_{4}$-treated patients with control subjects showed that despite similar TSH values the patients had significantly lower $T_{3}$ concentrations $(40,41,43)$. The log linear relationship between TSH and $\mathrm{T}_{3}$ was steeper (greater change in TSH for a given change in $\mathrm{T}_{3}$ ) in treated hypothyroid patients than in control individuals $(41,43)$. This has been interpreted by the authors as indicating an abnormal thyroid-pituitary feedback, with a reduced sensitivity compared to euthyroid controls. Finally, a recent retrospective study found that low TSH levels were necessary in order to achieve the same $\mathrm{T}_{3}$ levels during $\mathrm{LT}_{4}$ therapy that were observed in patients prior to their thyroidectomy (42). However, in this study patients underwent phlebotomy 2-4 hours after taking their $\mathrm{LT}_{4}$ tablets, leaving open the possibility that TSH concentrations were sampled during the trough of the diurnal TSH pattern, which could have resulted in lower TSH values than were present during the remainder of the day $(314,315,355)$. In addition, any comparison using the $\mathrm{T}_{4} / \mathrm{TSH}$ and $\mathrm{T}_{4} / \mathrm{T}_{3}$ relationship from the study data would be confounded by the fact that the investigators likely sampled the peak $\mathrm{T}_{4}$ levels. None of these studies examined patient satisfaction with therapy.

Another consideration in interpreting most of these studies is the rigor with which any population with confounding medications and co-existent illnesses were excluded because these factors are known to affect serum $\mathrm{T}_{3}$ concentrations (356). In addition, such studies should take patient age into 
account because not only does TSH increase with advancing age, but serum $\mathrm{T}_{3}$ levels decrease within older age groups as well (257).

In summary, all these studies show relatively high $\mathrm{FT}_{4}$ levels and either an increased $\mathrm{FT}_{4} / \mathrm{T}_{3}$ or $\mathrm{FT}_{4} / \mathrm{FT}_{3}$ ratio in $\mathrm{LT}_{4}$ treated patients. Whether these altered biochemical parameters have clinical consequences is unknown. For example, while excess $\mathrm{T}_{4}$ could in theory lead to increased $\mathrm{T}_{3}$ production in D2-expressing tissues, the regulation of D2 by the ubiquination-proteasomal system would tend to minimize this effect by inhibiting D2 activity and shortening its halflife (357). In addition, these studies differ with regard to the proportion of patients that can achieve normal $\mathrm{T}_{3}$ levels without lowering the serum TSH below the reference range. In one of the prospective studies, serum $\mathrm{T}_{3}$ levels were maintained as long as the serum TSH was less than $4.5 \mathrm{mIU} / \mathrm{L}$ (39). In one retrospective study, $15 \%$ of patients had serum $\mathrm{FT}_{3}$ concentrations that were below the levels in euthyroid controls (41). In another retrospective study, serum $\mathrm{FT}_{3}$ was only maintained if the patient's serum TSH was below $0.3 \mathrm{mIU} / \mathrm{L}$ (42). However, when taken together, several nonprospective studies do indicate that $\mathrm{LT}_{4}$ monotherapy is characterized by low $\mathrm{T}_{3}$ levels in either some or many patients (40-43). Whether these altered biochemical parameters have clinical consequences is unknown.

\section{7c. Is there evidence of discordance between the thyroid hormone status of different tissues and the serum thyrotropin concentration?}

\section{- Summary statement}

There are specific instances in which there appears to be discordance between the thyroid status of the pituitary gland, as reflected by the serum TSH, and the thyroid status of other tissues as indicated by various biomarkers. The clinical significance of this is not known.

\section{Mechanistic background}

Under normal physiological conditions, thyroid hormone signaling in the various organs and tissues is finely modulated at the target level, with different biological consequences. This diversity results from cells and tissues varying in terms of their expression of transmembrane transporters, deiodinases, nuclear TR subtypes, and coregulators (9,358,359). For example, the transporter MCT8 seems to be mostly important in the brain; D2 is expressed in the brain but not the liver; and the heart and skeleton are TR $\alpha$ predominant tissues whereas the liver is TR $\beta$ predominant. The consequences of differential deiodinase expression are striking: rodent studies indicate that the TRs in brain cells are almost fully occupied under normal conditions, whereas in the liver cells the receptors are only about half occupied (360). Furthermore, while nuclear $T_{3}$ content ultimately determines the intensity of the TR-mediated signaling, the set of genes affected by the TRs is cell type specific. Thus, systemic thyroid status is the integration of all of the individual thyroid statuses of the different cell types of the body at a given level of circulating $T_{3}$ and $T_{4}$.

Animal data strongly indicate that different tissues can have different concentrations of $T_{3}$, and thus different levels of thyroid hormone signaling at a given time; that is, tissues can have variable "tissue-specific thyroid status." Furthermore, normalization of the TSH does not ensure uniform $T_{3}$ levels in various tissues, though no data are available regarding the adequacy of thyroid signaling per se. This has been demonstrated in vitro by utilizing $L T_{4}$ infusion in animals, during which some tissues were demonstrated to be relatively $T_{3}$-deficient even though all were exposed to the same plasma $T_{3}$ and $T_{4}$ concentrations and in some cases had normal serum TSH levels (338,361). The best understood mechanism underlying tissue-specific thyroid status derives from the differential subcellular localizations of the deiodinases. While D2 and D3 are intracellular enzymes, D1 functions at the plasma membrane. Thus, D2 can directly alter the intracellular $T_{3}$ content without immediately altering plasma $T_{3}$ concentration. Thus, the differences in tissue distribution of the various forms of deiodinases result in tissue-specific supplies of $T_{3}$. In vitro data and rodent studies have established that primary changes in D2 or D3 in a given tissue can lead to tissue-specific changes in thyroid status without affecting circulating $T_{3}, T_{4}$, or TSH (362,363); human studies have not been reported.

\section{Discussion of the clinical literature}

In a 1971 study of myxedematous patients in whom therapy with $\mathrm{LT}_{4}$ was initiated, lowering of their elevated serum TSH values was shown to correlate with the dose of $\mathrm{LT}_{4}$ given (46). The serum TSH correlated well with both the administered dose $(r=-0.99)$ and the serum $\mathrm{FT}_{4}$ concentration achieved $(r=-1.0)$. In the years following, data have accumulated about the predictable (364), albeit complex (365-367), relationship between $\mathrm{FT}_{4}$ and TSH. Part of the complexity of this relationship is due to higher TSH values in older individuals, but at the same time a less robust response to hypothyroxinemia in older individuals, resulting in two overlapping sigmoidal curves (367). Serum TSH has been accepted as a robust, sensitive, and reproducible indicator of thyroid status (368). Serum TSH is thought to integrate signals from both $\mathrm{T}_{3}$ and $\mathrm{T}_{4}$ and its dynamic relationship with these parameters has led to its establishment as the best single test for determining systemic thyroid status. The logarithmic relationship between $\mathrm{TSH}$ and thyroid hormone bestows sensitivity: even if circulating $\mathrm{T}_{3}$ and $\mathrm{T}_{4}$ are in the normal range, it cannot be assumed that the subject is euthyroid. The interindividual ranges for $\mathrm{T}_{3}$ and $\mathrm{T}_{4}$ are much broader than the individual variance (369), such that measuring $\mathrm{T}_{3}$ and $\mathrm{T}_{4}$ is a suboptimal way to assess thyroid status.

Despite the time-honored concept that a normal serum TSH indicates that there is euthyroidism in all tissues, there are some data that do not support this concept. A caveat to using serum TSH as the sole indicator of systemic thyroid status can be proposed based on the observation that the TSH remains normal in some $\mathrm{LT}_{4}$-treated hypothyroid subjects with a high $T_{4}: T_{3}$ ratio, even when $T_{3}$ levels are lower than the normal range (39-43) (see section $7 \mathrm{~b}$ ). In these subjects, it is possible that in the pituitary, the low $\mathrm{T}_{3}$ is counterbalanced by the higher $\mathrm{T}_{4}$ (370). However, it is unknown whether all of the other tissues of the body, with their differing expression of deiodinases, transporters, and TRs, can maintain normal thyroid signaling in the face of this altered $T_{4}: T_{3}$ ratio, especially when $T_{3}$ is low. Other examples of situations in 
which $\mathrm{T}_{3}$ may be low in the face of normal serum TSH include starvation (371) and the polar $\mathrm{T}_{3}$ syndrome (372).

Other examples of discordant tissue response to therapy exist. In a study of daily monotherapy, a dose of $\mathrm{LT}_{3}$ that normalized cardiac parameters, serum cholesterol, serum creatine phosphokinase, and basal metabolic rate did not normalize TSH, which in fact remained elevated (370). A recent cross-over study of $\mathrm{LT}_{3}$ monotherapy and $\mathrm{LT}_{4}$ monotherapy that resulted in normal serum TSH concentrations during both arms of therapy was accompanied by weight loss, decreased cholesterol, and increased SHBG during the $\mathrm{LT}_{3}$ treatment phase (347). In another study in which resting energy expenditure was correlated with TSH levels, there were no concurrent TSH-associated changes in ACE concentration, SHBG levels, and the lipid profile (51). It has previously been suggested that $\mathrm{LT}_{4}$ therapy provides a signal of greater magnitude to the thyrotrophs than $\mathrm{LT}_{3}$ because $T_{3}$ is provided not only by the circulating levels but also from intrapituitary conversion of $\mathrm{T}_{4}$ to $\mathrm{T}_{3}$ (370). Other investigators, after performing TRH stimulation testing in patients receiving either $\mathrm{LT}_{4}$ or $\mathrm{LT}_{3}$ treatment reached a different conclusion, namely that differential responses to therapy were mediated peripherally and not at the pituitary gland level (373). Such studies indicate discordance between the signal emanating from the pituitary and other tissues.

A recent study examined the relationships between TSH, $\mathrm{FT}_{4}$, and $\mathrm{FT}_{3}$ in approximately 2000 patients who were either untreated or $\mathrm{LT}_{4}$ treated (374). Mathematical modeling showed different correlations between $\mathrm{TSH}$ versus $\mathrm{FT}_{4}$ and TSH versus $\mathrm{FT}_{3}$ in the treated and untreated groups. The authors noted that in subjects treated with $\mathrm{LT}_{4}$ monotherapy, the gradient between TSH and $\mathrm{FT}_{3}$ increased with $\mathrm{LT}_{4}$ dosage. They observed that increments of $\mathrm{LT}_{4}$ monotherapy were progressively more effective in suppressing TSH production, but simultaneously less effective in restoring $\mathrm{FT}_{3}$. They suggested that D2 levels within the pituitary are uniquely increased in order to maintain $\mathrm{T}_{3}$ production in the setting of high $\mathrm{T}_{4}$ levels (358), thus lowering TSH levels. The lowered TSH levels could subsequently result in failure to maintain $\mathrm{FT}_{3}$ levels. The authors suggested that their data and analyses $(374,375)$ provide evidence that TSH may not be the only standard for assessing euthyroidism and that $\mathrm{T}_{3}$ levels might need to be considered as well (374).

The clinical syndromes of resistance to thyroid hormone (RTH), caused by inactivating mutations in $\mathrm{TR} \alpha(\mathrm{RTH} \alpha)$ or $\operatorname{TR} \beta(\operatorname{RTH} \beta)$ could perhaps also be considered as a very particular example of how TSH may not necessarily reflect thyroid hormone signaling in other tissues than the pituitary and the hypothalamus. Patients with $\mathrm{RTH} \alpha$ have normal levels of TSH in combination with a low $\mathrm{FT}_{4}$ and high $\mathrm{T}_{3}$, and suffer from local hypothyroidism in tissues that predominantly express $\operatorname{TR} \alpha(376,377)$. Patients with $\operatorname{RTH} \beta$ have normal or elevated levels of TSH despite elevated levels of $\mathrm{FT}_{4}$ and $\mathrm{FT}_{3}$ and suffer from thyroid hormone overexposure in TR $\alpha$-expressing tissues $(378,379)$. Thus the molecular pathology of $\mathrm{RTH} \beta$ is an unbalanced hormonal signaling due to the combination of defective $\operatorname{TR} \beta$ and otherwise normal TR $\alpha$ receptors exposed to supraphysiologic levels of thyroid hormone. The result is a mix of hypothyroid symptoms and signs related to tissues such as the liver where the $\beta$ isoform is present in higher concentrations, and hyperthyroid symptoms and signs in tissues enriched with $\mathrm{TR} \alpha$ such as the myocardium (380).

\section{7d. Should levothyroxine therapy for hypothyroidism, particularly in specific subgroups such as those with obesity, depression, dyslipidemia, or who are athyreotic, be targeted to achieve high-normal triiodothyronine levels or low-normal thyrotropin levels?}

\section{- RECOMMENDATION}

There is insufficient evidence of benefit to recommend that treatment with levothyroxine be targeted to achieve lownormal thyrotropin values or high-normal triiodothyronine values in patients with hypothyroidism who are overweight, those who have depression or dyslipidemia, or those who are athyreotic.

Strong recommendation. Moderate quality of evidence.

\section{Discussion of the clinical literature}

Theoretically, it is possible that raising $\mathrm{T}_{3}$ levels to the upper part of the normal range or achieving TSH values in the lower part of the normal range during replacement for hypothyroidism may be beneficial for those with residual signs or symptoms that overlap with those of hypothyroidism, such as dyslipidemia, obesity, depression, or dissatisfaction with their therapy. Serum $\mathrm{T}_{3}$ would potentially be raised either by increasing a patient's $\mathrm{LT}_{4}$ dose to both lower the TSH and raise the $T_{3}$ concentration $(39,41-43)$, although feedback mechanisms would presumably limit the degree to which $\mathrm{T}_{3}$ could be raised. Alternatively $\mathrm{T}_{3}$ levels could be raised by use of combination therapy with $\mathrm{LT}_{3}$ (see discussion in section $13 b)$.

There are a handful of studies that potentially address the issue of raising $\mathrm{T}_{3}$ levels or lowering TSH levels by $\mathrm{LT}_{4}$ dosage adjustment in treated hypothyroid patients who have lipid disorders or depression, are dissatisfied with their therapy, or are overweight. In a recent study, hypothyroid patients were maintained at low-normal or high-normal TSH values for a year and had resultant $\mathrm{T}_{3}$ levels of 123 and $113 \mathrm{ng} / \mathrm{dL}$. This study showed no decrement in cholesterol, BMI, or percentage body fat associated with the higher $\mathrm{LT}_{4}$, despite an increase in resting energy expenditure (381). In a cross-over trial, 56 patients with primary hypothyroidism were treated for 8-week periods with three doses of $\mathrm{LT}_{4}$ in a random order, with achievement of TSH values of $2.8,1$, and $0.3 \mathrm{mIU} / \mathrm{L}$ and free $\mathrm{T}_{3}$ values of $2.5,2.7$, and $3.1 \mathrm{pg} / \mathrm{mL}$ respectively. There was progressive lowering of total cholesterol with the higher free $T_{3}$ values (50). However, the significance of this reduction seemed to be due to lowering of cholesterol in the group that included many patients with subclinical hyperthyroidism. Psychologic well-being, treatment satisfaction, and body weight did not change across the three different groups.

Two retrospective studies have examined weight changes in hypothyroid patients. One of these examined weight changes in individuals after either replacement or suppressive doses of $\mathrm{LT}_{4}$ for postsurgical hypothyroidism and found no significant weight changes (382). A second study compared weight changes during a 1-year period in patients who had 
just undergone thyroidectomy without ever becoming hypothyroid with patients already taking $\mathrm{LT}_{4}$ for primary hypothyroidism or thyroid cancer and also patients without a diagnosis of hypothyroidism (383). This study, in contrast, found those with hypothyroidism gained more weight than those without. The magnitude of the weight gain from greatest to least was (i) recently thyroidectomized, (ii) pre-existing hypothyroidism, and (iii) pre-existing thyroid cancer diagnosis. Although this latter study could be interpreted as showing that $\mathrm{LT}_{4}$ treatment to achieve a normal TSH fails to normalize some aspect of weight regulation, the cautions about drawing conclusions from retrospective studies applies.

A 2006 cross-sectional analysis examined correlations between psychological well-being, as assessed by General Health Questionnaire (GHQ) scores, and thyroid analytes in hypothyroid patients being treated with $\mathrm{LT}_{4}$. There was a relationship between both the log-transformed $\mathrm{TSH}$ and psychological well-being and also between $\mathrm{FT}_{4}$ concentrations and well-being, but no relationship existed between $\mathrm{FT}_{3}$ and well-being (67). In summary, these various studies, in which patients with AITD comprised $75 \%-80 \%$ of the population, suggest that dosage adjustments resulting in higher $\mathrm{T}_{3}$ concentrations do not decrease weight or BMI or improve patient well-being or satisfaction, but they may possibly decrease serum cholesterol.

Use of combination synthetic $\mathrm{LT}_{4}$ and $\mathrm{LT}_{3}$ therapy has not proven beneficial with respect to reducing body weight or improving lipid profile (see recommendation 13b), although analysis of subgroups with unfavorable lipid profiles or obesity have not been conducted. Symptoms such as anxiety and depression have not been shown to be less common in patients treated with combination therapy. Neither have patients with fatigue (384), dissatisfaction with their therapy (385), self-reported multidimensional psychopathology symptoms $(386)$, or depression $(387,388)$ been shown to respond better to combination therapy. However, small numbers of patients were available for some of these comparisons, for example four depressed patients versus 29 nondepressed patients in the study by Bunevicius et al. (387), and 14 patients with low fatigue levels and 14 with high fatigue levels in the study by Rodriguez et al. (384).

If there is a possibility of low $\mathrm{T}_{3}$ concentrations during $\mathrm{LT}_{4}$ therapy in the general population, and if the levels achieved are of concern, there may be another subpopulation for whom this theoretically is of even greater concern. This population would be patients with athyreosis. Those who are athyreotic as a result of thyroidectomy do not retain the ability to generate $\mathrm{T}_{3}$ from intrathyroidal sources, an ability that may be retained in patients with AITD who still have some endogenous function. The ability to assess whether athyreotic patients, in particular, benefit from combination therapy that includes $T_{3}$ is precluded by the small number of such patients who have been studied. Although the presence of athyreotic patients has been suggested to account for the positive outcome from one study of combination therapy (387), a metaanalysis performed in 2006 (389) did not find an association between positive outcomes from combination therapy and the athyreotic state. Another population who might potentially benefit from combination therapy is those patients who have the specific DIO2 polymorphism DIO2-Thr92Ala (390), although low serum $\mathrm{T}_{3}$ levels have not been documented in this subpopulation, and abnormal D2 activity has not been con- firmed in this population either. This topic is fully addressed in recommendation $13 \mathrm{~b}$.

\section{Levothyroxine Therapy in Secondary Hypothyroidism}

\section{8a. What biochemical goals should be employed for levothyroxine replacement in patients with secondary hypothyroidism?}

\section{- RECOMMENDATION}

In patients with secondary hypothyroidism, the primary biochemical treatment goal should be to maintain the serum free thyroxine values in the upper half of the reference range. However, the serum free thyroxine target level may be reduced in older patients or patients with comorbidities, who may be at higher risk of complications of thyroid hormone excess.

\section{Strong recommendation. Moderate quality evidence}

\section{Discussion of the clinical literature}

Secondary (central) hypothyroidism is characterized by insufficient pituitary production of TSH. This disorder affects $1: 100,000$ individuals and accounts for a small percentage of all cases of hypothyroidism (391). The treatment of secondary hypothyroidism and that of the more common primary hypothyroidism require different approaches based on the ability of the serum TSH concentration to serve as a reliable signaling mechanism $(391,392)$. Thus, in patients with primary hypothyroidism the serum TSH concentration is considered to be the best marker of thyroid status and is used as the basis for adjusting thyroid hormone dose. When the etiology of hypothyroidism is a pituitary deficit of TSH, this extremely sensitive and convenient indicator of thyroid status can, unfortunately, no longer be employed. Not only is serum TSH an unreliable indicator, but it may also become undetectable with small increments in $\mathrm{LT}_{4}$ that still leave the serum $\mathrm{FT}_{4}$ in the hypothyroid range (393).

The dilemma with respect to treating secondary hypothyroidism is that it cannot be determined what is the "normal" $\mathrm{FT}_{4}$ for the individual patient. Patients with an intact pituitary and subclinical thyroid dysfunction have, by definition, abnormal TSH values but $\mathrm{FT}_{4}$ values within the reference range. Subclinical thyroid dysfunction is well known to have adverse effects on lipid metabolism, the cardiovascular system, and the skeleton (4). Patients treated for secondary hypothyroidism could thus potentially be exposed to adverse effects of inadequate or excessive thyroid hormone without the usual signal from an abnormal TSH. Thus, an alternative measure of thyroid status may be required for treating secondary hypothyroidism.

Literature review revealed eight potentially relevant studies. Only one of these was a RCT, and this was of short duration (85). Six studies were retrospective examinations of groups of patients with hypothyroidism (394-399), and another was a prospective study of patients with secondary hypothyroidism alone, with no comparison group (393). These studies show that there are changes in physiologic parameters with dosage changes, but they were not specifically designed to address when a change in $\mathrm{LT}_{4}$ dose should be made. In addition, there are no long-term studies comparing 
different dosing of $\mathrm{LT}_{4}$, the resultant $\mathrm{FT}_{4}$ concentrations, and the subsequent outcomes in patients with central hypothyroidism.

The RCT crossed patients over from the "empiric" $\mathrm{LT}_{4}$ dose that had been selected by their treating physician (mean $1 \mu \mathrm{g} / \mathrm{kg} / \mathrm{d}$ ) to a body weight-adjusted dose of $1.6 \mu \mathrm{g} / \mathrm{kg} / \mathrm{d}$ (85). The dose of $1.6 \mu \mathrm{g} / \mathrm{kg} / \mathrm{d}$ produced higher $\mathrm{FT}_{4}$ levels that reached the upper part of the reference range, and it was associated with lower body weight, lower BMI, lower cholesterol (total, LDL, and high-density lipoprotein [HDL]), fewer clinical signs of hypothyroidism based on the Zulewski score, and a lower creatine kinase value. However, no differences in well-being and cognitive function were observed. The prospective study examined biochemical parameters in patients at three time points: when they were considered euthyroid on $\mathrm{LT}_{4}$, after discontinuing $\mathrm{LT}_{4}$, and then after resumption of $\mathrm{LT}_{4}$ (393). The $\mathrm{LT}_{4}$ dose used was the long-term treatment dose that had been selected by their prescribing physician. The mean dose was $1.5 \mu \mathrm{g} / \mathrm{kg}$, and this produced normal $\mathrm{FT}_{4}$ and $\mathrm{FT}_{3}$ values in most, but not all patients. Two out of 37 patients had low $\mathrm{FT}_{4}$ and $\mathrm{FT}_{3}$ levels, while 3 out of 37 patients had high $\mathrm{FT}_{3}$ concentrations while taking this dose. $\mathrm{LT}_{4}$ therapy was associated with significantly increased SHBG levels, ACE concentrations, and markers of bone turnover. No assessments of quality of life were made.

Some cross-sectional studies show that patients being treated for secondary hypothyroidism have lower $\mathrm{FT}_{4}$ values than patients being treated for primary hypothyroidism. One study compared $\mathrm{FT}_{4}$ values in patients being treated with $\mathrm{LT}_{4}$ for secondary hypothyroidism with values in patients being treated for primary hypothyroidism who had TSH values within the laboratory reference range (395). Approximately $20 \%$ of those with secondary hypothyroidism had a $\mathrm{FT}_{4}$ values below the 10th percentile, compared with less than $3 \%$ of patients with primary hypothyroidism (395). A study from 1999 found lower $\mathrm{FT}_{4}$ levels in treated patients with secondary hypothyroidism, compared with a group with primary hypothyroidism (398). Another study found that $1.6 \mu \mathrm{g} / \mathrm{kg}$ $\mathrm{LT}_{4}$ produced normal $\mathrm{FT}_{4}$ values in $94 \%$ of patients, but normal $\mathrm{FT}_{3}$ values in only $49 \%$ (394). The other retrospective studies show varying findings $(396,397)$.

The RCT described demonstrated that $\mathrm{FT}_{4}$ levels in the upper half of the reference range best reversed the clinical signs of hypothyroidism (85). A retrospective study also suggested that higher $\mathrm{FT}_{4}$ values within the normal range had most benefit on lipid parameters and body composition (399). Other recommendations in the literature include keeping $\mathrm{FT}_{4}$ concentrations within the reference range (391), keeping $\mathrm{FT}_{4}$ levels in the middle of the laboratory reference range (392, 393), and keeping the $\mathrm{FT}_{4}$ levels in the same range as the $\mathrm{FT}_{4}$ levels seen in patients being treated for primary hypothyroidism (395).

Only one study of combination therapy has been conducted in a population of patients with hypopituitarism (85). This was the randomized controlled cross-over study already discussed. Lower creatine kinase and faster ankle reflex time were seen with the combination therapy, compared with patients receiving $\mathrm{LT}_{4}$ at a dose of $1.6 \mu \mathrm{g} / \mathrm{kg}$, but the free $\mathrm{T}_{3}$ levels were supraphysiologic during combination therapy. The Zulewski score and working memory test were improved during combination therapy, but only compared with the $1.0 \mu \mathrm{g} / \mathrm{kg} / \mathrm{d}$, not the higher $1.6 \mu \mathrm{g} / \mathrm{kg}$ dose. Since there is in- sufficient evidence to recommend for combination therapy in patients with secondary hypothyroidism, no recommendation can be made regarding appropriate treatment goals.

As a separate observation, growth hormone replacement may necessitate either initiation of $\mathrm{LT}_{4}$ or an increase in the $\mathrm{LT}_{4}$ dose in patients with hypopituitarism (400). It has been suggested that growth hormone may enhance peripheral conversion of $\mathrm{T}_{4}$ to $\mathrm{T}_{3}$ and also have a central inhibitory effect on TSH release.

\section{8b. Are clinical parameters useful for assessing adequacy of levothyroxine replacement in patients with secondary hypothyroidism?}

\section{- RECOMMENDATION}

Although it may be helpful to follow changes in clinical parameters in patients treated for secondary hypothyroidism, such parameters alone lack sensitivity and specificity. There is a limited amount of evidence suggesting that clinical parameters are helpful as a secondary means of judging adequacy of replacement with levothyroxine in patients with secondary hypothyroidism in whom biochemical assessment is limited to serum free thyroxine levels.

\section{Weak recommendation. Moderate quality evidence}

\section{Discussion of the clinical literature}

Literature review revealed one potentially relevant study (85). This previously described RCT utilized a cross-over design with 29 patients either taking an "empiric" dose that had been selected by their treating physician (mean $1 \mu \mathrm{g} / \mathrm{kg}$ / d), a body weight-adjusted dose of $1.6 \mu \mathrm{g} / \mathrm{kg} / \mathrm{d}$ (85), or body weight adjusted combination therapy. This study examined body weight, BMI, ankle reflex time, Zulewski score, and neurocognitive testing to assess well-being and cognitive function on the three regimens. Both body weight-adjusted regimens were associated with higher $\mathrm{FT}_{4}$ levels, lower body weight, lower BMI, lower cholesterol (total, LDL, and HDL), and fewer clinical signs of hypothyroidism based on the Zulewski score. The only component of the neurocognitive test that differed between regimens was improved working memory in patients taking combination therapy. Ankle reflex time was only normalized by the combination therapy. In summary, clinical parameters such as body weight, BMI, and clinical score of hypothyroidism appear to mirror an increased dose of $\mathrm{LT}_{4}$ in a single small study.

\section{8c. Are tissue markers of thyroid hormone action helpful for management of levothyroxine replacement in patients with secondary hypothyroidism?}

\section{- RECOMMENDATION}

In patients with secondary hypothyroidism in whom the only available biochemical thyroid parameters are thyroid hormone levels, tissue markers of thyroid hormone action may be used, in addition to thyroid hormone parameters, as an adjunctive means of judging the adequacy of levothyroxine replacement.

Weak recommendation. Low-quality evidence. 


\section{Discussion of the clinical literature}

Literature review revealed two potentially relevant studies. One of these was the previously identified RCT (85). The other was a prospective study of 37 patients with secondary hypothyroidism undergoing reinstatement of their $\mathrm{LT}_{4}$ therapy (393). In the latter study $\mathrm{LT}_{4}$ therapy was associated with significantly increased concentrations of $\mathrm{FT}_{4}, \mathrm{FT}_{3}, \mathrm{SHBG}$, ACE, soluble interleukin-2 receptors, carboxyl-terminal telopeptides of type I collagen, and bone GLA protein. There was a significant positive correlation with $\mathrm{FT}_{3}$ concentration and the serum concentrations of soluble interleukin-2 receptors, carboxyl-terminal telopeptides of type I collagen, and bone GLA protein. The authors suggested that soluble interleukin-2 receptor concentration would be a useful parameter to monitor therapy because it was unaffected by concomitant therapy with growth hormone, sex steroids, and glucocorticoids. During the former randomized trial creatine kinase decreased in response to the higher thyroid hormone (TH) levels seen with the body weight-adjusted therapy. In summary, although raising $\mathrm{TH}$ levels in patients with secondary hypothyroidism correlated with biochemical parameters, no long-term studies of the use of these parameters to adjust therapy are available.

\section{Patient Satisfaction with Levothyroxine Therapy}

\section{9a. What tools may be useful in the clinical or research setting, to measure the impact of levothyroxine replacement for primary hypothyroidism on patients' physical or psychological well-being, treatment satisfaction, or treatment preferences?}

\section{- RECOMMENDATION}

Of the established instruments used to measure hypothyroid symptoms, data are lacking regarding their sensitivity and specificity in the "everyday" clinical setting to recommend their routine clinical use. Further studies are needed to determine if and how to combine general psychological screening instruments, hypothyroidism-specific tools, and laboratory assessment of thyroid function to measure the impact of levothyroxine replacement therapy on psychological well-being, treatment satisfaction, and preference in clinical practice. A combination of general instruments, combined with hypothyroidismspecific tools, may be the most effective way to examine psychological well-being in the levothyroxine-treated population in the research setting.

\section{Strong recommendation. Moderate quality evidence.}

\section{Discussion of the clinical literature}

Numerous organ systems are impacted by TH deficiency. Assessment of discrete end-organ responses to $\mathrm{LT}_{4}$ replacement in patients with primary hypothyroidism has focused on cardiovascular, neuromuscular, and cognitive measures. Assays of lipid parameters are commonly employed in both clinical and research settings, whereas echocardiography, cardiopulmonary exercise testing, and pulse wave velocity measures are used primarily in research studies. Likewise, neuromuscular and cognitive testing is usually restricted to the research setting. Numerous instruments have been applied to assess perceived health status, well-being, quality of life, and symptoms in research studies of $\mathrm{LT}_{4}$ replacement, though not all have been validated and some are not well suited for studying the impact of $\mathrm{LT}_{4}$ replacement, as reviewed by Razvi et al. (401). The Short-Form 36 (SF-36) (402) and the Nottingham Health Profile (403) have each been used to examine perceived health status and may be compared to normative data in other clinical populations. The SF-36 may be more sensitive to differences in the hypothyroid population (401). The GHQ (404) and Hospital Anxiety and Depression Scale (405) are general tools that measure psychological well-being.

Hypothyroidism-specific instruments have also been developed to measure health status [Chronic Thyroid Questionnaire (406)], quality of life [Underactive-ThyroidDependent Quality of Life Questionnaire $(407,408)$ and ThyPro (409)], and symptoms [Billewicz index (66), Zulewski score (58), Thyroid Symptom Questionnaire (68), Colorado Health Fair Thyroid Disease Symptom Survey (49), and Underactive Thyroid Treatment Satisfaction Questionnaire $(407,410)]$. These instruments have not been directly compared, so that the superiority of a specific instrument has not been determined. However, of those listed, only the Thyroid Symptom Questionnaire has not been validated. A combination of general instruments that can be compared to reference populations without thyroid disease and hypothyroidismspecific tools that are more sensitive to change may be the most effective way to examine psychological well-being in the $\mathrm{LT}_{4^{-}}$ treated population in the research setting.

\section{9b. What approach should be taken in patients treated for hypothyroidism who have normal serum thyrotropin values but still have unresolved symptoms?}

\section{- RECOMMENDATION}

A minority of patients with hypothyroidism, but normal serum thyrotropin values, may perceive a suboptimal health status of unclear etiology. Acknowledgment of the patients' symptoms and evaluation for alternative causes is recommended in such cases. Future research into whether there are specific subgroups of the population being treated for hypothyroidism who might benefit from combination therapy should be encouraged.

\section{Weak recommendation. Low-quality evidence.}

\section{Discussion of the clinical literature}

Two case-control studies have examined the question of residual hypothyroid symptoms in patients taking $\mathrm{LT}_{4}$. Saravanan et al. (68) conducted a case-control study in which 397 euthyroid $\mathrm{LT}_{4}$ users showed impaired psychological well-being and more thyroid symptoms compared to 551 ageand sex-matched controls. In this study, a survey was sent to both control patients not taking $\mathrm{LT}_{4}$ and patients taking $\mathrm{LT}_{4}$ who were matched for age and sex. More euthyroid patients being treated for hypothyroidism (34\%) had impairment in their sense of psychological well-being (GHQ score $\geq 3$ ) compared to controls (25\%). Approximately $10 \%$ more hypothyroid patients were dissatisfied based on a thyroid symptom questionnaire. The differences remained significant even after adjustment for other medical conditions, which were generally more prevalent in the group receiving 
treatment for hypothyroidism. This was not confirmed in a smaller, similarly designed case-control study from Eskelinen et al. (411). However, a third study also showed more fatigue in euthyroid patients than in euthyroid controls (412). A fourth study, in which subjects were recruited by letter and then subsequently took part in cognitive testing and completed questionnaires assessing well-being, also showed lower scores in both these areas in the euthyroid patients taking $\mathrm{LT}_{4}$, but the comparison was with standard reference values, rather than a formal control group (413). The difficulty in interpreting these types of studies is that their findings are highly dependent on the health of the control group, the fact that recruitment methods may elicit a higher response rate from individuals who are dissatisfied, and the likelihood that patients with hypothyroidism are highly attuned to potential symptoms of hypothyroidism.

Three studies have addressed the relationship between symptoms of hypothyroidism and serum TSH levels in $\mathrm{LT}_{4}$ users. A cross-sectional analysis of 697 patients taking $\mathrm{LT}_{4}$ showed a correlation between higher TSH levels and more hypothyroid symptoms, even within the subset of 470 patients who were euthyroid (67). On the other hand, another cross-sectional study did not find a relationship between wellbeing and two categories of TSH values of less than and greater than $2 \mathrm{mIU} / \mathrm{L}$ (413). Moreover, in a randomized, controlled cross-over trial by Walsh et al. (50) of three different $\mathrm{LT}_{4}$ doses that achieved TSH levels below the lower limit of the reference range, in the lower half of the reference range, and in the upper half of the reference range, there were no differences in symptoms or significant treatment preferences. This study suggests that $\mathrm{LT}_{4}$ users do not experience symptomatic benefit from lower serum TSH levels.

It has been argued that the serum TSH level should not be the sole way to determine euthyroidism, and that clinical symptoms should be incorporated into the determination of the optimal $\mathrm{LT}_{4}$ dose (414). In a study by Carr et al. (415), patients had symptomatic improvement on an $\mathrm{LT}_{4}$ dose that was $50 \mu \mathrm{g} / \mathrm{d}$ higher than their optimal dose and resulted in low serum TSH levels. However, it should be noted that this was a nonrandomized, open-label study of short duration and therefore subject to participant bias. In summary, patients treated with $\mathrm{LT}_{4}$ to euthyroid serum TSH goals may experience more symptoms of hypothyroidism than people who do not have treated hypothyroidism. However, data from one randomized clinical trial show that increasing the $\mathrm{LT}_{4}$ dose does not affect these symptoms.

The question that these studies do not answer is why some patients with hypothyroidism have residual symptoms despite serum TSH levels that fall within the reference range. Inadequacy of $\mathrm{LT}_{4}$ to resolve hypothyroid symptoms has been invoked to explain residual symptoms. However, other explanations should be considered. For example, there are studies suggesting that the presence of thyroid peroxidase antibodies per se, even in patients who have maintained endogenous euthyroidism, may be associated with more symptoms than are reported by euthyroid patients without thyroid autoimmunity. One study found more symptoms and lower quality of life in patients with elevated titers of TPOAb (416), whereas another study reported more depression (417). Simply being labeled as a patient with hypothyroidism could be associated with feeling unwell. In addition, it has been shown that individuals referred for thyroid testing by their primary care physicians had rates of psychological distress that were approximately twice as high as the general population under the care of a physician, with 54\% versus $19 \%$ having GHQ scores $>3$ (418). This was despite the fact that the rate of hypothyroidism in the referred patients was not higher than that in the general population. Thus, it is more likely that those with psychological distress will have SCH not only detected, but also treated, even if the psychological distress is not causally related to $\mathrm{SCH}$. Moreover, symptoms thought to indicate the presence of hypothyroidism, but not actually related to the hypothyroid state, will presumably not resolve with the application of $\mathrm{LT}_{4}$.

Even though serum $\mathrm{T}_{3}$ concentrations are typically not measured during an evaluation of hypothyroidism, if they are in fact measured, it is theoretically appealing from a physiological standpoint to attempt to normalize them, along with other biochemical parameters. A recent survey of practice patterns found that $22 \%$ of clinicians would measure serum $\mathrm{T}_{3}$ levels when confronted by a biochemically euthyroid patient with persistent symptoms (419). No published studies have demonstrated that relatively low $\mathrm{T}_{3}$ levels have negative consequences for patients with normal serum TSH values, so this is an, as yet, untested hypothesis. If symptomatic patients, who are euthyroid based on their serum TSH values, also have relatively low serum $\mathrm{T}_{3}$ levels during $\mathrm{LT}_{4}$ therapy (39-43) in the absence of nonthyroidal illness, they could be considered for referral for future clinical trials of $\mathrm{LT}_{3}$ containing combination therapy (see discussion in recommendation 13 and Future Directions). As practicing clinicians, we are aware that $T_{3}$ therapy is being administered to selected patients under these circumstances. The previously mentioned survey of practice patterns found that $3.6 \%$ of physicians would prescribe $\mathrm{LT}_{3}$ under such circumstances (419). A recent review discussed the addition of $\mathrm{LT}_{3}$ to $\mathrm{LT}_{4}$ therapy in persistently symptomatic patients with biochemical euthyroidism under carefully monitored conditions (420). A trial of $\mathrm{LT}_{4} / \mathrm{LT}_{3}$ therapy is mentioned in the 2012 ATA thyroid hormone treatment patient brochure. An individual trial of $\mathrm{T}_{3}$ therapy to normalize the serum $\mathrm{T}_{3}$ levels without driving serum TSH below the normal range is unsupported by data and therefore is an unproven approach, but, following extensive discussion, some task force members thought it could be considered on an individual basis. This would be considered innovative therapy, which occurs when a practitioner uses a treatment in a way that deviates from commonly accepted practice. When such new treatments are used repeatedly, they should be made the object of formal research at an early stage, in order to determine whether the innovation is both safe and effective. When $\mathrm{T}_{3}$ therapy is used outside of a trial it is difficult to determine whether the therapy had the desired response in the individual patient and the patient's uncontrolled experience does not contribute to determining the effectiveness of the therapy.

\section{Use of Levothyroxine in Euthyroid Individuals}

\section{0a. Is there a role for the use of levothyroxine to treat biochemically euthyroid patients with symptoms that overlap with those of hypothyroidism?}

\section{- RECOMMENDATION}

We strongly recommend against the use of levothyroxine treatment in patients who have nonspecific symptoms and 
normal biochemical indices of thyroid function because no role exists for use of levothyroxine in this situation.

\section{Strong recommendation. High-quality evidence.}

\section{Discussion of the clinical literature}

In a randomized placebo controlled cross-over trial in patients with symptoms suggestive of hypothyroidism but biochemical euthyroidism, $\mathrm{LT}_{4}$ was no more effective than placebo in improving cognitive and psychological wellbeing scores despite increases in serum $\mathrm{FT}_{4}$ and decreases in serum TSH when study subjects were receiving $\mathrm{LT}_{4}$ (421). A study employing 12 months of TSH suppression therapy in euthyroid patients with thyroid nodules did not detect any changes in parameters such as weight, fat mass, waist circumference, and exercise performance compared with patients untreated with $\mathrm{LT}_{4}$, suggesting that $\mathrm{LT}_{4}$ also does not alter body composition or muscle function in euthyroid individuals (422). In part, the negative outcomes of these studies may be explained by the finding that symptoms do not always accurately predict hypothyroidism. In one case-control study of patients identified through laboratory records, when patients with biochemical hypothyroidism were compared with euthyroid individuals, there did not seem to be specific symptoms that separated one group from another (57). The number of symptoms experienced by patients may be helpful in detecting hypothyroidism: hypothyroid patients had seven symptoms or more and were also more likely to have experienced a change in their symptoms (57). In another study, a symptom score correlated well with Achilles reflex time and cholesterol, but not TSH (58). Based on these data, there does not appear to be a substantiated role for the use of $\mathrm{LT}_{4}$ therapy to treat symptoms in biochemically euthyroid patients with symptoms that overlap with those of hypothyroidism. Furthermore, there may be harm in treating euthyroid patients with $\mathrm{LT}_{4}$ due to potential iatrogenic hyperthyroidism and potential deflection of attention away from any underlying nonthyroidal disorders, including somatization disorders.

\section{0b. Is there a role for the use of levothyroxine to treat euthyroid patients with depression?}

\section{- RECOMMENDATION}

We recommend against the routine use of levothyroxine for the treatment of euthyroid individuals with depression due to a paucity of controlled data examining treatment efficacy in this setting.

\section{Weak recommendation. Low-quality evidence.}

\section{Discussion of the clinical literature}

Individuals referred for thyroid testing are frequently found to have symptoms of psychologic distress (418). However, there is no simple association between a diagnosis of hypothyroidism and depression. Most individuals with depression do not exhibit signs or symptoms of hypothyroidism (423); similarly most individuals with hypothyroidism do not exhibit symptoms of depression (424). Although overall the incidence of hypothyroidism does not appear to be greater in those with depression, depression may be found more often in those previously diagnosed with hypothyroid- ism than in those newly diagnosed with hypothyroidism (424). When the thyroid function of depressed patients was compared with controls, those with depression had a slightly higher TSH (1.9 versus $1.5 \mathrm{mIU} / \mathrm{L})$ (425). Overall, the association between depression and thyroid dysfunction is relatively weak (424) and could potentially be due to the thyroid disease itself, unmasking of depression following treatment of thyroid dysfunction, or coping with a chronic medical illness.

Depression is seen in some overtly hypothyroid patients (426) and usually resolves with treatment (427). On the other hand, depression is not more common in patients with $\mathrm{SCH}$ $(428,429)$, and when it does co-exist with mild hypothyroidism, $\mathrm{LT}_{4}$ therapy does not improve it (430).

To our knowledge, there are no controlled studies of $\mathrm{LT}_{4}$ therapy in depression. Data from open-label studies using supraphysiologic doses of $\mathrm{LT}_{4}(250-500 \mu \mathrm{g} / \mathrm{d})$ in bipolar disorders $(431,432)$ and refractory depression (433) have yielded positive results. However, there are no randomized placebo controlled trials of $\mathrm{LT}_{4}$ therapy for bipolar disorder or depression, with or without concomitant antidepressant therapy. One randomized trial of $\mathrm{TH}$ potentiation of tricyclic antidepressant nonresponders compared $37.5 \mu \mathrm{g}$ daily of $\mathrm{LT}_{3}$ to $150 \mu \mathrm{g}$ daily of $\mathrm{LT}_{4}$, and found greater responses to $\mathrm{LT}_{3}$ compared to $\mathrm{LT}_{4}$, but the study had no placebo arm (434). See section 15a for a more detailed discussion on the use of $\mathrm{LT}_{3}$ in euthyroid patients with depression.

\section{0c. Is there a role for the use of levothyroxine to treat euthyroid patients with obesity?}

\section{- RECOMMENDATION}

We recommend against the treatment of obesity with levothyroxine in euthyroid individuals due to a lack of treatment efficacy for this condition

\section{Strong recommendation. Moderate quality evidence.}

\section{Discussion of the clinical literature}

Obesity rates are increasing in the United States $(435,436)$, possibly contributed to by an abundant food supply and sedentary lifestyles. Cross-sectional studies have demonstrated increasing BMI or body weight associated with increasing TSH values (437-439), but autoimmunity itself may be linked with obesity (440), or obesity may be the trigger for changes in thyroid parameters $(441,442)$, rendering difficulty in assigning cause and effect. Moreover, increased TSH values seen in obese patients may revert to normal after weight loss (442). Although hypothyroidism is often perceived to be a cause of obesity by the public, most of the weight gain (and weight loss with therapy) in TH deficiency states is due to fluid retention. There is no significant loss of fat mass, even after therapy of severe hypothyroidism, despite increases in resting energy expenditure (443). Similarly, there are no significant changes in body weight after $\mathrm{LT}_{4}$ treatment of SCH $(84,444)$. Although THs have been used as a means to attempt to induce weight loss in the past [e.g., Rivlin (445)], negative nitrogen balance (446) and other deleterious effects on the heart and bone (234-236) dictate against the use of $\mathrm{TH}$ to treat obesity. Furthermore, a 
recent meta-analysis has shown that $\mathrm{LT}_{4}$ is an ineffective treatment for obesity (447).

\section{0d. Is there a role for the use of levothyroxine to treat euthyroid patients with urticaria?}

\section{- RECOMMENDATION}

We recommend against the treatment of urticaria with levothyroxine in euthyroid individuals due to a lack of treatment efficacy for this condition.

\section{Strong recommendation. Moderate quality evidence.}

Chronic urticaria is often seen in patients with underlying AITD, especially Hashimoto's thyroiditis. Approximately $25 \%$ of patients with chronic idiopathic urticaria seem to have evidence of thyroid autoimmunity (448). Anecdotal reports have suggested that $\mathrm{LT}_{4}$ therapy may be useful in the treatment of urticaria in euthyroid individuals with positive thyroid autoantibodies [e.g., Monge et al. (449)]. However, a retrospective study did not show any benefit of $\mathrm{LT}_{4}$ treatment that restored euthyroidism in hypothyroid patients with AITD, compared to patients with urticaria without AITD, when the activity of the urticaria in both groups was monitored for 6 months (450). A small, randomized trial showed no improvement with $\mathrm{LT}_{4}$ when taken with an antihistamine in euthyroid patients with AITD compared to an antihistamine alone (451). Other studies in the literature are generally underpowered and uncontrolled (448).

\section{0e. What is the recommended approach to treating factitious thyrotoxicosis?}

\section{- RECOMMENDATION}

Factitious thyrotoxicosis should be treated with discontinuation of the exogenous thyroid hormone with education and/or psychiatric consultation as appropriate.

\section{Strong recommendation. Low-quality evidence.}

\section{Discussion of the clinical literature}

Factitious thyrotoxicosis (thyrotoxicosis factitia) has traditionally been defined as a syndrome wherein patients surreptitiously ingest TH (452). It can be associated with Münchausen's syndrome (in which physical symptoms and signs of illness are intentionally produced) and is also associated with neuroses related to poor body image and concerns about increased body weight. More recently, the term factitious thyrotoxicosis has been expanded (453) to include instances of accidental $\mathrm{LT}_{4}$ ingestion, such as in pediatric poisoning or pharmacy error, or through intentional or unintentional ingestion of supplements that contain thyroid gland extract (454). The treatment is the discontinuation of TH. In individuals with psychiatric disorders who are secretly taking $\mathrm{TH}$, psychiatric consultation is warranted. Such a consult may not only provide relief for the underlying disorder, but also serves to protect the patient from thyrotoxicrelated events. Factitious thyrotoxicosis has not been studied in a systematic manner. A strong recommendation is made despite low-quality evidence because there are benefits, but few risks.

\section{Clinical Ethics Considerations in Levothyroxine Treatment}

\section{What are the ethical obligations of clinicians in treating hypothyroidism?}

\section{- RECOMMENDATION}

Clinical ethical principles in levothyroxine treatment for hypothyroidism revolve around two core ethical principles in medicine: the Principles of Beneficence and NonMaleficence, which guide the risk/benefit analysis in clinical practice, and protect clinicians from deviating from practice to satisfy inappropriate patient demands. Additional ethical obligations revolve around the professional virtues of competence and intellectual honesty.

\section{Ungraded.}

\section{Discussion of the clinical ethics literature}

Clinical ethics is a field of practice that refers to the "bedside" ethical issues and dilemmas that arise in the everyday delivery of patient care. Among the four core medical ethical principles introduced and codified in the 1970s in most developed countries, the relevant ethical principle in the treatment of hypothyroidism is the Principle of Beneficence, which guides health care providers to maximize clinical benefits and minimize clinical harms; and the Principle of Non-Maleficence, the obligation to not intentionally introduce harms to patients or to not intentionally initiate a therapy that is known to have no therapeutic benefit. The Principle of Non-Maleficence also directs practitioners to warn third parties of imminent harms, if the patient appears to be a threat to an identifiable third party or the public (455); this is also a legal standard, codified in health law doctrine.

Offering patients formulations of THs or other preparations that are known to be inferior to the standard of care, potentially futile, or even harmful contravenes the Principles of Beneficence and Non-Maleficence. Failing to fulfill the Duty to Warn, for example, by notifying third parties or authorities when severely hypothyroid patients are driving and are known threats to public safety or even child safety (if driving children), is a violation of Non-Maleficence (455).

Most practitioners are taught that patient autonomy means that they should cater to patient preferences. However, if patients are requesting inappropriate therapies, this is a misinterpretation. The Principle of Respect for Persons is a principle that dually guides practitioners to respect patient autonomy, but to also protect patients who do not have full decision-making capacity from pointless harm. Autonomy can only be enabled with valid informed consent, which comprises full disclosure and explanations of all procedures and treatments; decision-making capacity, which means that patients must demonstrate understanding, appreciation, rationality, and expression of a choice; and voluntariness, in which there is no coercion.

Goals of care should be guided by autonomous patients' preferences, but there are limits to what practitioners may offer if patients are demanding therapies that are outside the standard of care or potentially harmful. In the context of hypothyroidism, patients may express a preference to feel 
well and be restored to euthyroid levels, yet refuse synthetic $\mathrm{LT}_{4}$ therapy because it is not "natural." This stated preference could indicate the patient does not understand and appreciate the pharmacologic properties of $\mathrm{LT}_{4}$, which can be explained as restoring natural physiologic function. In this common example, the patient's preference can be understood as a preference not to have drug side effects or be harmed. Respecting the patient's preference, in this context, would be to ensure the patient has a truer understanding of hypothyroidism and $\mathrm{LT}_{4}$ action; only when the patient understands and appreciates that choosing thyroid extract therapy rather than $\mathrm{LT}_{4}$ could expose them to certain risks such as elevated serum $T_{3}$ levels would this constitute informed refusal of standard therapy. If there is inability of autonomous patients to demonstrate understanding, appreciation, rationality, and expression of a choice, this indicates there is a barrier to decision-making capacity, and thus, valid informed consent cannot be claimed. Several articles provide more information about informed consent, decision-making capacity, and surrogacy in the thyroidology context $(456,457)$.

Beneficent care thus recognizes there are limits to patient autonomy when patients or their surrogates request substandard, unsound, or untested medical procedures or therapies that could be either futile or harmful. It should be recognized that while autonomous patients may accept or refuse therapies, they should not be abandoned to "autonomy" when they seek therapies that violate beneficent care (458-460) by being provided with inappropriate care because they request it. When Beneficence and NonMaleficence are violated, there may be legal consequences and licensure revocation because these ethical violations constitute medical negligence. Two recent judgments in high-profile medical negligence cases $(461,462)$ involved practitioners acceding to patients' demands for unsound medical therapies. In these cases, the physicians abandoned primary obligations to beneficence and non-maleficence, and treated the patients as their customers. In one judgment, the practitioner was stripped of his medical license $(461,463)$; in the other, he was convicted of involuntary manslaughter for iatrogenic harms caused by a therapy the patient demanded (462).

All medical practitioners have professional ethical duties to be competent in their fields of practice, which includes intellectual honesty $(464,465)$ and referring patients to other colleagues in areas in which knowledge is insufficient. Practitioners who state they are experts in treating hypothyroidism when they have no demonstrable accredited training or board certification (e.g., "integrated medicine") violate professional ethical standards of practice because it overtly deceives patients. Additionally, competently trained medical experts who misuse their medical knowledge to personally profit, deceive patients, or purvey nonstandard, risky innovative therapies in hypothyroidism are violating basic standards of care.

Finally, there are special ethical considerations for prenatal and pediatric contexts. For more information about these special populations, as well as the full range of clinical and professional ethical considerations relevant in thyroidology, we refer clinicians to the Clinical and Professional Ethics Guidelines published by the ATA Ethics Advisory Committee (456) and a recent review article (457).

\section{SECTION II. THERAPIES OTHER THAN LEVOTHYROXINE ALONE}

\section{Thyroid Extracts}

\section{In adults requiring thyroid hormone replacement treatment for primary hypothyroidism, is treatment with thyroid extracts superior to treatment with levothyroxine alone?}

\section{- RECOMMENDATION}

We recommend that levothyroxine be considered as routine care for patients with primary hypothyroidism, in preference to use of thyroid extracts. Although there is preliminary evidence from a short-duration study that some patients may prefer treatment using thyroid extracts, high-quality controlled long-term outcome data are lacking to document superiority of this treatment compared to levothyroxine therapy. Furthermore, there are potential safety concerns related to the use of thyroid extracts, such as the presence of supraphysiologic serum triiodothyronine levels and a paucity of long-term safety outcome data.

\section{Strong recommendation. Moderate quality evidence.}

\section{Mechanistic background}

The thyroid gland secretes a variety of iodinated and noniodinated molecules that collectively play important roles during prenatal and adult lives. Understanding what these molecules are and what they do informs our understanding of the therapy for hypothyroidism. The main noniodinated molecule secreted by the thyroid is calcitonin, an endogenous hormone with probable effects on calcium and bone metabolism. The other iodine-containing molecules include $T_{4}, T_{3}$, $r T_{3}, 3,3^{\prime}$-diiodothyronine, 3,5-diiodothyronine (3,5- $\left.T_{2}\right)$, monoiodothyronine, and their decarboxylated forms known as thyronamines.

While the traditional view has been that $T_{3}$ is important as the main iodothyronine with significant binding to nuclear TRs at physiological concentrations, and $T_{4}$ is important as a precursor of $T_{3}$ (466), the use of desiccated thyroid extracts for therapy of hypothyroidism is based on the hypothesis that other molecules present in the thyroid gland may have important effects. A growing number of in vitro studies have indicated that administration of pharmacologic doses of 3,5$\mathrm{T}_{2}$ has metabolic effects, possibly mediated via nongenomic mechanisms (467-469). Note that the mechanism by which 3,5-T $T_{2}$ is generated in humans is unclear; furthermore, the circulating levels of 3,5-T, is two orders of magnitude lower than $T_{3}(470,471)$. Finally, thyronamines have been shown to interact with the G-protein coupled receptor transcript antisense to ribosomal RNA (TAR1), with 3-iodothyronamine being the most potent agonist. In pharmacologic doses, 3-iodothyronamine triggers diverse effects such as hypothermia, behavioral inactivity, bradycardia, and decreased cardiac output. Metabolic effects such as a shift from carbohydrate to fat metabolism and stimulation of food intake have also been described (472).

In summary, current evidence supports the classical view that $T_{3}$ and $T_{4}$ are the only biologically important secreted products of the thyroid; none of the alternate signaling molecules have been definitely shown to have physiologic relevance in humans at endogenous concentrations. It is not 
known whether oral use of thyroid extracts would provide any of these "alternate" components to patients, since studies of their retention during product preparation, their absorption into the blood from the gastrointestinal tract, and subsequent concentrations achieved have not been published. The available literature does suggest that pharmacologic administration of 3,5-T $T_{2}$ and iodothyronamines could have metabolic effects.

\section{Discussion of the clinical literature}

Desiccated thyroid or thyroid extract refers to preparations that are derived from the thyroid gland of animals. These preparations were the primary therapy for hypothyroidism until the advent of synthetic $\mathrm{T}_{4}$ preparations in the 1960s. All commercially available prescription desiccated preparations are derived from pigs. As per the United States Pharmacopeia (USP), desiccated thyroid is "the cleaned, dried, and powdered thyroid gland previously deprived of connective tissue and fat. It is obtained from domesticated animals that are used for food by humans." Tablets are measured for $T_{4}$ and $T_{3}$ content and are formulated into doses expressed as "grains" with 1 grain $(65 \mathrm{mg})$ tablets containing $38 \mu \mathrm{g}$ of $\mathrm{T}_{4} ; 9 \mu \mathrm{g}$ of $\mathrm{T}_{3}$; protein-bound iodine; and unmeasured quantities of diiodothyronine, monoiodothyronine, and calcitonin, to which inactive ingredients are added for tablet stability. Varying strengths from fractions to multiples of a grain are commercially available. One process used to achieve specific dosage strengths is to mix different batches of the product during manufacture. The bioavailability of the $T_{4}$ and $T_{3}$ components has been shown to be the same between desiccated thyroid and synthetic preparations (473).

There are two main clinical concerns with the use of desiccated thyroid preparations, both of which center on their $\mathrm{T}_{3}$ component. The ratio of $\mathrm{T}_{4}$ to $\mathrm{T}_{3}$ in desiccated thyroid preparations is $4.2: 1$, which is significantly lower than the 14:1 ratio of secretion by the human thyroid gland. This relative excess of $T_{3}$ leads to supraphysiologic levels of $T_{3}$ (474-477). In addition, due to the shorter half-life of $\mathrm{T}_{3}$, fluctuations of $\mathrm{T}_{3}$ occur over the course of the day, with peak levels shortly after dosing $(475,477)$. Thus, there is concern for thyrotoxicosis if thyroid extract therapy is not adjusted according to the serum TSH. One nonrandomized cross-over study documented a larger number of hyperthyroid symptoms when individuals were taking desiccated thyroid prep- arations compared with levothyroxine preparations, which was attributed to $\mathrm{T}_{3}$ effects (474).There are claims from proponents of each type of therapy regarding the superiority of their preparation $(478,479)$. However, there is only one randomized clinical trial comparing desiccated thyroid to $\mathrm{LT}_{4}$ in the therapy of hypothyroidism (480) (see Table 6). Although this cross-over study did suggest that in selected individuals there was patient preference for the extract and minimal weight loss associated with its use, the study, which was designed as a superiority trial, and powered for quality of life endpoints, not safety endpoints, was essentially a negative study in that improved quality of life was not also seen over the 16 weeks of treatment.

It is worth noting that when evaluating health outcomes, measures such as quality of life and mortality are clearly health outcomes $(481,482)$. However, demonstration of patient preference without use of a validated questionnaire may not truly be a health outcome and traditionally has not been considered to be a health outcome (482). In addition, the preference outcome may need to be interpreted in the context of another validated health outcome, such as quality of life. There was also no routine documentation of the daily excursion in $\mathrm{T}_{3}$ concentration that was associated with thyroid extract use (only two patients had serum $\mathrm{T}_{3}$ levels measured on one occasion 3 hours after taking the thyroid extract) (480), thus not providing data to counter the older studies (474-477) showing hypertriiodothyroninemia $2-5$ hours after thyroid extract use. The clinical consequences of such serum $\mathrm{T}_{3}$ excursions are unknown. These high $\mathrm{T}_{3}$ levels may be of particular concern in patients receiving suppressive therapy for thyroid cancer using a thyroid extract.

There are no published controlled long-term outcome trials of the use of desiccated thyroid extract. Furthermore, data from studies of combinations of $\mathrm{LT}_{4}$ and $\mathrm{LT}_{3}$ therapy (as reviewed in recommendation 13b) do not suggest additional benefit from the addition of $\mathrm{LT}_{3}$ to $\mathrm{LT}_{4}$ replacement therapy. However, the long-term therapeutic efficacy of the $T_{4}: T_{3}$ ratio found in porcine desiccated thyroid and of the remaining components of porcine thyroids found in these tablets has not been assessed, leaving the direct benefits and risks of desiccated thyroid therapy untested. Moreover, use of thyroid extract should not be contemplated during pregnancy. Delivery of $\mathrm{T}_{4}$ is thought to be crucial for the developing fetal brain (483) and using a $\mathrm{TH}$ preparation in which the $\mathrm{T}_{4}$ concentration may be lower than that in $\mathrm{LT}_{4}$ could be deleterious.

Table 6. Characteristics of the Randomized Controlled Trial Comparing Health and Psychological Effects in Hypothyroid Patients Treated with Thyroid Extract (Containing $\mathrm{T}_{4}$ and $\mathrm{T}_{3}$ ) Compared to $\mathrm{LT}_{4}$ Alone

\begin{tabular}{|c|c|c|c|c|c|c|}
\hline Reference & $\begin{array}{l}\text { Treatment } \\
\text { dosing }^{\mathrm{a}}\end{array}$ & $\begin{array}{c}\text { Etiology primary } \\
\text { hypothyroidism }\end{array}$ & Design & $\begin{array}{l}\text { No. of patients } \\
\text { randomized } \\
\text { (completed } \\
\text { follow-up) }\end{array}$ & $\begin{array}{l}\text { Treatment } \\
\text { duration }\end{array}$ & $\begin{array}{l}\text { End of study } \\
\text { TSH differences } \\
\text { between groups }\end{array}$ \\
\hline 480 & $\begin{array}{l}\mathrm{T}_{4} \text { : usual } \\
\text { Thyroid extract: } 1 \mathrm{mg} \\
\text { extract for each } \\
1.667 \mu \mathrm{g} \text { usual } \mathrm{T}_{4} \\
\text { Dosing: once daily }\end{array}$ & $\begin{array}{l}\text { Mixed: autoimmune, } \\
\text { post-RAI, } \\
\text { thyroid surgery, } \\
\text { post-EBRT, } \\
\text { no thyroid cancer }\end{array}$ & $\begin{array}{l}\text { Randomized, } \\
\text { double blind } \\
\text { cross-over study }\end{array}$ & $78(70)$ & 16 weeks & $\begin{array}{l}\text { Thyroid } \\
\quad \text { extract }>\mathrm{LT}_{4}\end{array}$ \\
\hline
\end{tabular}

\footnotetext{
${ }^{\text {a }}$ Starting treatment doses are shown (prior to adjustments within trials according to thyroid function tests).
}

EBRT, external beam radiation therapy; RAI, radioactive iodine. 
Synthetic Combination Therapy and the Rationale for Its Use

13a. Do genetic variants in thyroid hormone pathway genes (deiodinases or thyroid hormone transporters) affect the serum or tissue levels of thyroid hormones in healthy euthyroid individuals or hypothyroid patients taking replacement therapy?

\section{- Summary statement}

Specific polymorphisms in the deiodinases are consistently associated with very small changes in serum thyroid hormone levels. Insufficient data exist to draw any conclusion about the clinically relevant effects of deiodinase or transporter polymorphisms on tissue thyroid hormone levels.

Mechanistic background

Twin and family studies have shown that between $26 \%$ and $65 \%$ of the variation in serum thyroid function tests is due to genetic factors $(484,485)$. Genetic variants can be divided into two broad classes, based on their frequency and their functional effects on the target gene. Polymorphisms are common genetic variants that occur frequently in the population and generally have only very modest effects on gene function. In contrast, mutations are rare genetic variants with major effects on gene function leading to monogenic disorders such as $R T H \alpha, R T H \beta$ (see section $7 c$ and section 24 ), or the Allan-Herndon-Dudley syndrome due to mutations in the TH transporter MCT8 (486). While no patients have been identified with functionally important deiodinase mutations, seven patients with mutations in SECISBP2, a gene critical for the synthesis of selenocysteine containing proteins including the deiodinases, have been identified. Affected individuals have a mild phenotype but do exhibit decreased serum $T_{3}$ levels (487).

Candidate gene studies have been widely used to study associations of polymorphisms in TH pathway genes with thyroid function tests. Such studies are straightforward to perform, and can be powered to detect small effects of specific alleles. Care must be taken to avoid false-positive results arising from small sample size, lack of replication in an independent cohort, lack of standardized phenotyping and genotyping, and population stratification when insufficient care has been paid to matching cases and controls (488). Alternatively, genome-wide association studies (GWAS) can be performed with genotyping of large numbers of polymorphisms across the genome rather than focusing on a single candidate gene. GWAS studies require much larger sample sizes than candidate gene studies in order to have adequate statistical power.

In recent years, it has been demonstrated that certain polymorphisms are consistently associated with serum TSH and iodothyronine levels. A recent large meta-analysis of GWAS for serum levels of TSH and $\mathrm{FT}_{4}$ in up to 26,420 and 17,520 euthyroid subjects, respectively, identified 26 independent associations. Some were in loci that had previously been identified in candidate gene analyses, but many novel loci that were significantly associated with $\mathrm{TSH}$ and $\mathrm{FT}_{4}$ were identified as well (489). Together, these genetic markers explain $5.64 \%$ of the trait variation in TSH, and $2.30 \%$ of the variation in $\mathrm{FT}_{4}$, suggesting that many other genetic markers remain to be identified.

Evaluating whether mutations or polymorphisms are important for a protein's function can be done in cells or with mouse studies in which the altered gene is knocked into the germline of the animal. Such studies may be limited because of the possibility of cell-specific or species-specific effects, and also because proteins may have functions unknown to the investigators that are therefore overlooked. The effects on protein function have not been established for most of the polymorphisms associated with TSH and $\mathrm{FT}_{4}$, not even for the most consistent single nucleotide polymorphisms (SNPs) in the type D1 gene (DIO1) (489,490).

Similarly, in vitro studies have as yet failed to reveal any consistent functional effect of the widely studied D2 gene (DIO2) polymorphism (rs225014) that results in a Thr92Ala substitution in the enzyme (491). The polymorphism is located in an instability loop of the protein and about 9\%$16 \%$ of the population is homozygous for the substitution (492). The D2 Thr92Ala variant is associated with decreased D2 enzyme velocity in skeletal muscle and thyroid tissue samples of patients with type 2 diabetes mellitus who were homozygous for the variant (493). In contrast, cells transiently expressing the Thr92Ala form of D2 display similar kinetic properties with either $T_{4}$ or $r T_{3}$ as substrate as compared to controls $(493,494)$. This being the case, the clinical associations with this polymorphism may result from an unstudied function of the polymorphism or from linkage disequilibrium with another unidentified locus in the genome.

On the other hand, another D2 polymorphism with reported clinical associations, the DIO2-258A/G (rs12885300) was associated with an increased ratio of $T_{3} / T_{4}$, consistent with an increase in deiodinase activity (495). In vitro studies suggest that this polymorphism may cause an increase in D2 expression (496). Pharmacogenomic studies designed to interrogate the role of the DIO2-Thr92Ala (497) and the DIO2-258A/G (498) polymorphisms on the hypothalamicpituitary-thyroid axis indicate that these polymorphisms have distinct, small, but significant modulatory effects on the TSH secretion and on the pattern of TH secretion. Homozygous subjects showed a delayed rise in serum $T_{3}$ (497) and a blunted rise in $F_{4}$ and normal $T_{3}$ release (498), respectively, indicating subtle alterations in intrathyroidal conversion of $T_{4}$ into $T_{3}$, with opposite effects on the $T_{3}$ product, associated with these two polymorphisms.

The D1 polymorphisms DIO1-C785T (rs11206244) and DIO1-A1814G (rs12095080) appear to be associated with decreased and increased activity of D1, respectively, possibly by altering mRNA stability or folding (499). It should be noted that data are lacking as to whether polymorphisms in TH pathway genes, including the deiodinases and transmembrane transporters, are also associated with differences in tissue $T_{3}$ levels. In the case of D2, which is critical for local $\mathrm{T}_{3}$ generation, studies in rodents with inactivating $\mathrm{D} 2 \mathrm{mu}$ tations have shown $T_{3}$ deficiency in D2-dependent tissues such as the brain (339-341). Thus, the possible effects of genetic variants in $D 2$ on tissue $T_{3}$ levels in human subjects should be a subject of future research.

Similarly, effects of polymorphisms in TH transporters on transport function and thus tissue $T_{3}$ levels have not been reported. The possibility that a genetic transport abnormality 
caused by a polymorphism could be physiologically relevant seems possible based on the observation that inactivating mutations of MCT8 in hemizygous males have been found to cause severe psychomotor retardation, known as the AllanHerndon-Dudley syndrome. These individuals have peripheral hypermetabolism and abnormal serum thyroid levels with elevated serum $T_{3}$, low serum $T_{4}$, and slightly high TSH (486,500,501).

\section{Discussion of the clinical literature}

A number of studies have looked for associations between deiodinase polymorphisms and differences in circulating TH levels. While some polymorphisms are associated with statistically significant differences across genotypes, these differences are very small, such that $T_{3}, T_{4}$, and $\mathrm{rT}_{3}$ remain in their respective normal ranges $(489,495,502,503)$. For example, the DIO1-C785T polymorphism has been associated with a small decrease in serum $\mathrm{T}_{3}$, trending from $131.8 \mathrm{ng} / \mathrm{dL}$ (CC) to $127.7 \mathrm{ng} / \mathrm{dL}$ (TT) (502). The C allele of the DIO1 polymorphism rs2235544 has, on the other hand, been associated with an increase in the $\mathrm{FT}_{3}$ concentration in both $\mathrm{LT}_{4}$ treated and nontreated individuals in a meta-analysis (504) and the D1-A1814G allele is associated with a higher $\mathrm{T}_{3} / \mathrm{rT}_{3}$ ratio (494). The best-studied polymorphism in DIO2 (DIO2- Thr92Ala) is, in fact, not associated with any change in circulating $\mathrm{TH}$ levels (502), while another $\mathrm{DIO} 2$ polymorphism DIO2-258A/G (D2-ORFa-Gly3Asp) was associated with lower serum $\mathrm{T}_{4}$ and $\mathrm{FT}_{4}$ levels and an increased $\mathrm{T}_{3} /$ $\mathrm{T}_{4}$ ratio, but was not associated with serum $\mathrm{T}_{3}$ levels (495).

A few studies have investigated whether genetic variants in the TH pathway genes (i.e., the deiodinases) are associated with altered function of the thyroid axis in human patients taking $\mathrm{LT}_{4}$ monotherapy. For example, when present as the homozygous Ala/Ala form, the Thr92Ala polymorphism has been associated with a higher requirement for $\mathrm{LT}_{4}$ in order to achieve near-suppression of serum TSH in patients who have undergone total thyroidectomy (224). However, a subsequent study found no effect of the Thr92Ala D2 polymorphism on the $\mathrm{LT}_{4}$ dose needed to achieve TSH suppression in thyroid cancer patients or TSH normalization in patients with Hashimoto's hypothyroidism (222). The relationship between $\mathrm{FT}_{4}$ and TSH in patients appears to be affected by their DIO2258A/G (DIO2-ORFa-Gly3Asp) polymorphism status. The negative feedback of $\mathrm{FT}_{4}$ on TSH is weaker in homozygous patients being treated with $\mathrm{LT}_{4}$ (505). Given this limited amount of data, it remains to be proven that these D2 polymorphisms have a clinically important effect on $\mathrm{TH}$ economy.

A large number of studies have looked for associations between genetic variants in the TH pathway genes and other clinical syndromes. For example, a recent GWAS identified 22 polymorphisms that were associated with serum $\mathrm{TSH}$, four of which were also significantly associated with the risk of thyroid cancer (506). Most studies however, have focused on the role of the DIO2-Thr92Ala D2 polymorphism, which was initially identified as being clinically relevant in a study associating it with insulin resistance and increased BMI (491). Subsequently, this polymorphism has been associated with various phenotypes including mental retardation (507), hypertension (508), osteoarthritis (509), accelerated bone turnover (510), and response to lung injury (511), although subsequent studies could not confirm the associations with hypertension (512). Ultimately, understanding the connections between the deiodinase polymorphisms and these other entities will depend upon the discovery of an enzyme property affected by the polymorphism, or an effect on tissue levels of $\mathrm{T}_{3}$ conveyed by these polymorphisms, or linkage to another critical genetic locus or loci.

MCT8 has also been studied to determine whether there is any impact of polymorphisms on serum $\mathrm{TH}$ parameters. Results from these studies are mixed with associations between SNPs and $\mathrm{FT}_{4}$ and $\mathrm{FT}_{3}$ shown in one study (513), but not in others (30).

\section{3b. In adults requiring thyroid hormone replacement treatment for primary hypothyroidism, is combination treatment including levothyroxine and liothyronine superior to the use of levothyroxine alone?}

\section{- RECOMMENDATION}

There is no consistently strong evidence of superiority of combination therapy over monotherapy with levothyroxine. Therefore, we recommend against the routine use of combination treatment with levothyroxine and liothyronine as a form of thyroid replacement therapy in patients with primary hypothyroidism, based on conflicting results of benefits from randomized controlled trials comparing this therapy to levothyroxine therapy alone and a paucity of long-term outcome data.

Weak recommendation. Moderate quality evidence.

\section{3c. In adults requiring thyroid hormone} replacement treatment for primary hypothyroidism who feel unwell while taking levothyroxine, is combination treatment including levothyroxine and liothyronine superior to the use of levothyroxine alone?

\section{- RECOMMENDATION}

For patients with primary hypothyroidism who feel unwell on levothyroxine therapy alone (in the absence of an allergy to levothyroxine constituents or an abnormal serum thyrotopin), there is currently insufficient evidence to support the routine use of a trial of a combination of levothyroxine and liothyronine therapy outside a formal clinical trial or $N$-of-1 trial, due to uncertainty in long-term risk benefit ratio of the treatment and uncertainty as to the optimal definition of a successful trial to guide clinical decision-making. Additional research targeting those with relatively low serum triiodothyronine concentrations, but normal thyrotropin levels during monotherapy is needed to address whether there is a subgroup of patients who might benefit from combination therapy.

\section{Insufficient evidence}

\section{Mechanistic background}

A fundamental characteristic of D2 is that its enzymatic activity is tightly coupled to plasma $T_{4}$ levels by the ubiquitinproteasomal system (358). As circulating $T_{4}$ falls in hypothyroid subjects, the fractional conversion rate of $T_{4}$-to- $T_{3}$ increases (514). In hypothyroid rodents, this increase occurs 
via a PTU-insensitive pathway (515) (i.e., D2). The flux of $T_{4}$ through the D2 activation pathway increases due to increased enzyme activity, such that serum $T_{3}$ levels remain normal, at least until the fall in serum $T_{4}$ is substantial. In D2expressing tissues such as the brain, much of the intracellular $T_{3}$ is generated from circulating $T_{4}(340,516) ;$ thus, these tissues are particularly protected from mild $T_{4}$ deficiency. The existence of the D2 pathway for conversion of $T_{4}$ to $T_{3}$, and the homeostatic function of $D 2$ for maintenance of intracellular $T_{3}$ in the face of mild $T_{4}$ deficiency, explain why $L_{4}$ monotherapy can be so effective in the treatment of hypothyroidism. Treatment with PTU in patients reliant on $\mathrm{LT}_{4}$ therapy reduces their serum $T_{3}$ levels significantly by about $20 \mathrm{ng} / \mathrm{dL}$. Presumably this reduction is due to inhibition of the $P T U$-susceptible D1, illustrating both that D1 contributes in part to maintenance of serum $T_{3}$ and that D2 contributes importantly to maintenance of serum $T_{3}$ levels (19).

At the same time, data indicate that a small but significant proportion of thyroidectomized patients on $\mathrm{LT}_{4}$ monotherapy have low circulating $T_{3}$ in spite of normal range TSH and high-normal serum $T_{4}$ (39-43). It is possible that in these subjects, the D2 pathway does not fully compensate for the absence of the thyroidal $T_{3}$ production; the significance of the low serum $T_{3}$ levels in these patients is discussed in section $7 a$ and $7 b$. Note that even D2-expressing tissues would be expected to be relatively $T_{3}$ deficient since $D 2$ is not upregulated significantly by decreases in serum $T_{3}$. The serum TSH remains in the normal range likely because the decreased serum $T_{3}$ is counterbalanced by the increased serum $T_{4}$. If this concept is correct, the question then becomes how to simultaneously normalize the concentration of $T_{3}$ in all tissues? Increasing $L_{4}$ dose would raise serum $T_{3}$, thus raising tissue $T_{3}$ content in all tissues, but at the cost of lowering serum TSH, and at the theoretical risk of inducing mild hyperthyroidism in D2-expressing tissues. Hypothetically, replacing some of the administered $L T_{4}$ dose with $L T_{3}$ could help normalize tissue $T_{3}$ without necessarily lowering serum TSH. Data supporting the concept that combination therapy can be effective in normalizing tissue $T_{3}$ across all tissues are available from rodent studies (361), but corresponding data in humans are not available. Animal studies have shown that some tissues are relatively $T_{3}$-deficient during $\mathrm{LT}_{4}$ infusions (338,361). However the cerebral cortex was an exception, as in this tissue $T_{3}$ homeostasis was maintained over a wide range of $T_{4}$ infusions (338). An infusion of a combination of $L T_{4}$ and $L T_{3}$ allowed for normalization of serum TSH and levels of $T_{4}$ and $T_{3}$ in all tissues tested (361).

\section{Discussion of the clinical literature}

In order to evaluate whether the combination of $\mathrm{LT}_{4}$ with $\mathrm{LT}_{3}$, also referred to as combination therapy, was clinically superior to $\mathrm{LT}_{4}$ alone, in $\mathrm{TH}$ replacement of adults with primary hypothyroidism, we reviewed the results of 13 original RCTs (384-388,517-524), as well as four systematic reviews or meta-analyses of selected RCTs $(389,525-527)$. The reason for reviewing all original studies, rather than only the published reviews or meta-analyses, was that some trials were published after these reviews or meta-analyses were conducted. We did not include a randomized trial published by Smith et al. (528) in 1970, since the dosages of $\mathrm{LT}_{4}$ that were used were higher than those typically used in current clinical practice and the study predated utilization of TSH measurements, both of which may have contributed to $\mathrm{TH}$ overreplacement in the study. We did not include a controlled cross-over design trial from Regalbuto et al. (529), given that there was no evidence of randomization of patients reported in the methods section of the article.

The characteristics of the included original trials are described in Table 7. The studies included a variety of $\mathrm{LT}_{4} / \mathrm{LT}_{3}$ dose combinations, administered either once or twice a day, and the duration of treatments ranged from 5 weeks to 1 year, with the majority of studies being 6 months or shorter in treatment duration (Table 7). The majority of patients in most studies had primary autoimmune hypothyroidism, but some studies included patients who had thyroid surgery or radioactive iodine treatment (Table 7). Females accounted for the vast majority of participants studied in all of the studies $(80 \%$ or more of the study population in respective trials) (384388,517-524). Some trials provided information regarding pretrial screening for relevant symptoms such as depression and fatigue using validated questionnaires (404,530-533) and described inclusion/exclusion criteria based on these symptoms (Table 8). All included trials had some level of blinding, except for one study focusing on laboratory parameter outcomes by Fadayev et al. (520). Of the 13 included trials comparing the effect of combination $\mathrm{LT}_{4} / \mathrm{LT}_{3}$ treatment to $\mathrm{LT}_{4}$ alone, seven had a cross-over design $(384,385,387$, $517,519,521,523)$, and the rest had a parallel design $(386,388,518,520,522,524)$. The impact of combination $\mathrm{LT}_{4} /$ $\mathrm{LT}_{3}$ treatment on serum TSH levels measured at study completion was as follows: (i) no significant difference compared to $\mathrm{LT}_{4}$ therapy alone-eight studies $(384,387,388,517,518$, $520,521,524)$; (ii) significantly higher TSH measurement in the combination therapy group compared to the $\mathrm{LT}_{4}$ groupthree studies $(385,519,522)$; or (iii) significantly lower TSH measurement in the combination therapy group compared to $\mathrm{LT}_{4}$ alone-two studies $(386,523)$, including one study in which the serum TSH in the highest dose combination group (5:1 ratio of $\mathrm{LT}_{4}$ to $\mathrm{LT}_{3}$ ), was suppressed to $0.07 \mathrm{mIU} / \mathrm{L}$ (386).

Among the 12 trials examining health-related quality of life or mood (384-388,517-519,521-524), there was marked unexplained clinical heterogeneity of $\mathrm{LT}_{4} / \mathrm{LT}_{3}$ treatment effects, categorized as follows: (i) marked superiority of combination treatment on multiple measures-two trials $(387,521)$, (ii) superiority of combination treatment on a minority of measurements-two trials, with one trial from Saravanan et al. (522) reporting some improvements in mood at 3 months but not 12 months, and another trial from Valizadeh et al. (524) reporting improvement of an insomnia/ anxiety subscale with other measures not significantly different; or (iii) no statistically significant superiority of combination treatment over $\mathrm{LT}_{4}$ treatment - eight trials (384$386,388,517-519,523)$. It is important to note that it was not uncommon for psychological or quality of life measurements to improve in both treatment groups, suggesting an important placebo effect. Among the 10 trials reporting measures of neurocognitive functioning or behavior, there was marked unexplained clinical heterogeneity of $\mathrm{LT}_{4} / \mathrm{LT}_{3}$ treatment effect, with the results summarized as follows: (i) marked superiority of combination treatment on multiple measures-one trial by Bunevicius et al. (387), (ii) superiority of combination treatment on a minority of measurements-one trial by 
Table 7. Characteristics of Randomized Controlled Trials Comparing Health and Psychological Effects in Hypothyroid Patients Treated with Synthetic Combination $\mathrm{LT}_{4} / \mathrm{LT}_{3}$ Therapy Compared to $\mathrm{LT}_{4}$ Alone

\begin{tabular}{|c|c|c|c|c|c|c|}
\hline Reference & $\begin{array}{l}\text { Treatment } \\
\text { dosing }^{\mathrm{a}}\end{array}$ & $\begin{array}{l}\text { Etiology primary } \\
\text { hypothyroidism }\end{array}$ & Design & $\begin{array}{l}\text { No. of patients } \\
\text { randomized } \\
\text { (completed } \\
\text { follow-up) }\end{array}$ & $\begin{array}{l}\text { Treatment } \\
\text { duration }\end{array}$ & $\begin{array}{l}\text { End of study } \\
\text { TSH differences } \\
\text { between groups }\end{array}$ \\
\hline 386 & $\begin{array}{l}\mathrm{T}_{4}: \text { usual dose } \\
\mathrm{LT}_{4} / \mathrm{LT}_{3}: 10: 1 \text { or } \\
5: 1 \text { ratio of } \\
\mathrm{T}_{4} \text { to } \mathrm{T}_{3} \text { ratio, } \\
\text { respectively } \\
\text { Dosing: twice daily }\end{array}$ & Autoimmune & $\begin{array}{l}\text { Parallel, } \\
\text { blinded }\end{array}$ & $141(130)$ & 15 weeks & $\begin{array}{l}\mathrm{LT}_{4} / \mathrm{LT}_{3}<\mathrm{LT}_{4} \\
\text { (TSH } \\
\text { significantly } \\
\text { lower only } \\
\text { in the } 5: 1 \\
\mathrm{LT}_{4} / \mathrm{LT}_{3} \\
\text { dose group) }\end{array}$ \\
\hline 387 & $\begin{array}{l}\mathrm{T}_{4} \text { : usual } \\
\mathrm{LT}_{4} / \mathrm{LT}_{3} \text { : usual } \\
\mathrm{T}_{4} \text { dose minus } \\
50 \mu \mathrm{g} / \mathrm{d} \text { with } \\
\mathrm{T}_{3} 12.5 \mu \mathrm{g} / \mathrm{d} \\
\text { Dosing: once daily }\end{array}$ & $\begin{array}{l}\text { Mixed: autoimmune, } \\
\text { thyroid cancer }\end{array}$ & $\begin{array}{l}\text { Cross-over, } \\
\text { blinded }\end{array}$ & $35(33)$ & 5 weeks & NS \\
\hline 517 & $\begin{array}{l}\mathrm{T}_{4} \text { : usual } \\
\mathrm{LT}_{4} / \mathrm{LT}_{3} \text { : usual } \\
\mathrm{T}_{4} \text { dose minus } \\
50 \mu \mathrm{g} / \mathrm{d} \text { with } \\
\mathrm{T}_{3} 10 \mu \mathrm{g} / \mathrm{d} \\
\text { Dosing: once daily }\end{array}$ & $\begin{array}{l}\text { All Graves' disease, } \\
\text { history of subtotal } \\
\text { thyroidectomy }\end{array}$ & $\begin{array}{l}\text { Cross-over, } \\
\text { blinded }\end{array}$ & $13(10)$ & 5 weeks & NS \\
\hline 518 & $\begin{array}{l}\mathrm{T}_{4} \text { : usual } \\
\mathrm{LT}_{4} / \mathrm{LT}_{3} \text { : usual } \\
\mathrm{T}_{4} \text { dose minus } \\
50 \mu \mathrm{g} / \mathrm{d} \text { with } \mathrm{T}_{3} \\
15 \mu \mathrm{g} / \mathrm{d} \\
\text { Dosing: twice daily }\end{array}$ & $\begin{array}{l}\text { Mixed: autoimmune, } \\
\text { post-RAI, thyroid } \\
\text { surgery, post- } \\
\text { EBRT, 1 patient } \\
\text { thyroid cancer }\end{array}$ & $\begin{array}{l}\text { Parallel, } \\
\text { blinded }\end{array}$ & $46(44)$ & 4 months & NS \\
\hline 519 & $\begin{array}{l}\mathrm{T}_{4}: 100 \mu \mathrm{g} / \mathrm{d} \\
\mathrm{LT}_{4} / \mathrm{LT}_{3}: \mathrm{LT}_{4} \\
75 \mu \mathrm{g} / \mathrm{d} \text { and } \\
\mathrm{T}_{3} 5 \mu \mathrm{g} / \mathrm{d} \\
\text { Dosing: once daily }\end{array}$ & $\begin{array}{l}\text { Mixed: autoimmune, } \\
\text { post-RAI }\end{array}$ & $\begin{array}{l}\text { Cross-over, } \\
\text { blinded }\end{array}$ & $28(26)$ & 8 weeks & $\mathrm{LT}_{4} / \mathrm{LT}_{3}>\mathrm{LT}_{4}$ \\
\hline 520 & $\begin{array}{l}\mathrm{T}_{4}: 1.6 \mu \mathrm{g} / \mathrm{kg} / \mathrm{d} \\
\mathrm{LT}_{4} / \mathrm{LT}_{3}: \text { estimated } \\
\mathrm{T}_{4} \text { dose minus } \\
25 \mu \mathrm{g} / \mathrm{d} \\
\text { with } \mathrm{T}_{3} 12.5 \mu \mathrm{g} / \mathrm{d} \\
\text { Dosing: once daily }\end{array}$ & $\begin{array}{l}\text { Untreated overt } \\
\text { hypothyroidism, } \\
\text { etiology } \\
\text { not reported }\end{array}$ & $\begin{array}{l}\text { Parallel, } \\
\text { unblinded }\end{array}$ & $36(36)$ & 6 months & NS \\
\hline 521 & $\begin{array}{l}\mathrm{T}_{4} \text { : usual } \\
\mathrm{LT}_{4} / \mathrm{LT}_{3} \text { : usual } \\
\mathrm{T}_{4} \text { dose minus } \\
50 \mu \mathrm{g} / \mathrm{d} \text { with } \\
\mathrm{T}_{3} 20 \text { or } 50 \mu \mathrm{g} / \mathrm{d} \text {, } \\
\text { respectively } \\
\text { Dosing: once daily }\end{array}$ & Autoimmune & $\begin{array}{l}\text { Cross-over, } \\
\text { blinded }\end{array}$ & $68(59)$ & 12 weeks & NS \\
\hline 384 & $\begin{array}{l}\mathrm{T}_{4}: \text { usual } \\
\mathrm{LT}_{4} / \mathrm{LT}_{3}: \text { usual } \\
\mathrm{T}_{4} \text { dose minus } \\
50 \mu \mathrm{g} / \mathrm{d} \text { with } \\
\mathrm{T}_{3} 10 \mu \mathrm{g} / \mathrm{d} \\
\text { Dosing: once daily }\end{array}$ & $\begin{array}{l}\text { Mixed: autoimmune, } \\
\text { post-RAI, } \\
\text { thyroid surgery }\end{array}$ & $\begin{array}{l}\text { Cross-over, } \\
\text { blinded }\end{array}$ & $30(27)$ & 6 weeks & NS \\
\hline 522 & $\begin{array}{l}\mathrm{T}_{4}: \text { usual } \\
\mathrm{LT}_{4} / \mathrm{LT}_{3}: \text { usual } \\
\mathrm{T}_{4} \text { dose minus } \\
50 \mu \mathrm{g} / \mathrm{d} \text { with } \\
\mathrm{T}_{3} 10 \mu \mathrm{g} / \mathrm{d} \\
\text { Dosing: once daily }\end{array}$ & $\begin{array}{l}\text { Primary } \\
\text { hypothyroidism, } \\
\text { no thyroid cancer }\end{array}$ & $\begin{array}{l}\text { Parallel, } \\
\text { blinded }\end{array}$ & $697(573)$ & $\begin{array}{l}12 \text { Months } \\
\text { (outcomes } \\
\text { assessed } 3 \\
\text { and } 12 \text { months) }\end{array}$ & $\mathrm{LT}_{4} / \mathrm{LT}_{3}>\mathrm{LT}_{4}$ \\
\hline
\end{tabular}


TABle 7. (CONTINUED)

\begin{tabular}{|c|c|c|c|c|c|c|}
\hline Reference & $\begin{array}{l}\text { Treatment } \\
\text { dosing }^{\mathrm{a}}\end{array}$ & $\begin{array}{c}\text { Etiology primary } \\
\text { hypothyroidism }\end{array}$ & Design & $\begin{array}{l}\text { No. of patients } \\
\text { randomized } \\
\text { (completed } \\
\text { follow-up) }\end{array}$ & $\begin{array}{l}\text { Treatment } \\
\text { duration }\end{array}$ & $\begin{array}{l}\text { End of study } \\
\text { TSH differences } \\
\text { between groups }\end{array}$ \\
\hline 388 & $\begin{array}{l}\mathrm{T}_{4} \text { : usual } \\
\mathrm{LT}_{4} / \mathrm{LT}_{3} \text { : usual } \\
\mathrm{T}_{4} \text { dose minus } \\
50 \mu \mathrm{g} / \mathrm{d} \text { with } \\
\mathrm{T}_{3} \text { total } 25 \mu \mathrm{g} / \mathrm{d} \\
\text { Dosing: Twice daily } \\
\mathrm{T}_{3} \text {, once daily } \mathrm{T}_{4}\end{array}$ & $\begin{array}{l}\text { Primary } \\
\text { hypothyroidism, } \\
\text { no thyroid cancer, } \\
\text { no thyroidectomy, } \\
\text { no history of } \\
\text { hyperthyroidism }\end{array}$ & $\begin{array}{l}\text { Parallel, } \\
\text { blinded }\end{array}$ & $40(33)$ & 15 weeks & NS \\
\hline 523 & $\begin{array}{l}\mathrm{T}_{4} \text { : usual } \\
\mathrm{LT}_{4} / \mathrm{LT}_{3} \text { : usual } \\
\mathrm{T}_{4} \text { dose minus } 5 \% \\
\text { with } \mathrm{T}_{3} 5 \% \\
\text { (aim } 14: 1 \text { ratio } \\
\mathrm{LT}_{4} \text { to } \mathrm{T}_{3} \text { ) } \\
\text { Dosing: once daily }\end{array}$ & $\begin{array}{l}\text { Mixed: autoimmune, } \\
\text { post-RAI, thyroid } \\
\text { surgery, no } \\
\text { thyroid cancer }\end{array}$ & $\begin{array}{l}\text { Cross-over, } \\
\text { blinded }\end{array}$ & $26(23)$ & 12 weeks & $\mathrm{LT}_{4} / \mathrm{LT}_{3}<\mathrm{LT}_{4}$ \\
\hline 524 & $\begin{array}{l}\mathrm{T}_{4}: \text { usual } \\
\mathrm{LT}_{4} / \mathrm{LT}_{3} \text { : usual } \\
\mathrm{T}_{4} \text { dose minus } \\
50 \mu \mathrm{g} / \mathrm{d} \text { with } \\
\mathrm{T}_{3} \text { total } 12.5 \mu \mathrm{g} / \mathrm{d} \\
\text { Dosing: twice daily } \\
\mathrm{T}_{3}, \mathrm{~T}_{4} \text { once daily }\end{array}$ & $\begin{array}{l}\text { Mixed: autoimmune, } \\
\text { post-RAI, thyroid } \\
\text { surgery, but } \\
\text { no one on TSH- } \\
\text { suppressive therapy }\end{array}$ & $\begin{array}{l}\text { Parallel, } \\
\text { blinded }\end{array}$ & $71(60)$ & 4 months & NS \\
\hline 385 & $\begin{array}{l}\mathrm{T}_{4} \text { : usual } \\
\mathrm{LT}_{4} / \mathrm{LT}_{3} \text { : usual } \\
\mathrm{T}_{4} \text { dose minus } \\
50 \mu \mathrm{g} / \mathrm{d} \text { with } \\
\mathrm{T}_{3} 10 \mu \mathrm{g} / \mathrm{d} \\
\text { Dosing: once daily }\end{array}$ & $\begin{array}{l}\text { Mixed: autoimmune, } \\
\text { post-RAI, thyroid } \\
\text { surgery, but no } \\
\text { thyroid cancer on } \\
\text { TSH-suppressive } \\
\text { therapy }\end{array}$ & $\begin{array}{l}\text { Cross-over, } \\
\text { blinded }\end{array}$ & $110(101)$ & 10 weeks & $\mathrm{LT}_{4} / \mathrm{LT}_{3}>\mathrm{LT}_{4}$ \\
\hline
\end{tabular}

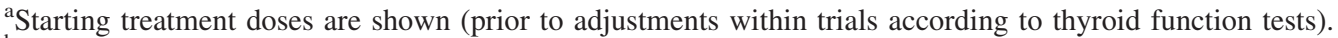

${ }^{\mathrm{b}}$ Dosing not reported, assume once daily.

NS, not significantly different; RAI, radioactive iodine treatment; EBRT, external beam radiation treatment.
}

Escobar-Morreale et al. (519), and (iii) no statistically significant superiority of combination treatment over $\mathrm{LT}_{4}$ treatment—eight trials (384-386,388,517,518,522,523), including a study by Clyde et al. (518), in which cognitive performance on a Grooved Peg Board test was better in the $\mathrm{LT}_{4}$ group, compared to the combination therapy.

Thyroid-related symptoms and thyroid-related health outcomes are clinically relevant outcomes examined in many of the included trials. There was no significant difference in thyroid-specific symptom questionnaires between treatment groups in the seven trials measuring these outcomes $(384,385,517-520,522)$. Of the nine trials examining the outcome of body weight (384-386,517-519,521,522,524), the end of trial weight was significantly reduced in the combination therapy group compared to the $\mathrm{LT}_{4}$ group in only one trial at a TSH-suppressive dose of combination therapy ( $\mathrm{LT}_{4}: \mathrm{LT}_{3}$ 5:1 ratio) (386), with no significant differences observed in the other studies $(384,385,517-519,521,522,524)$. Bone mineral densitometry, measured after 6 months of treatment, was not significantly different with combination treatment compared to $\mathrm{LT}_{4}$ alone in one study of premenopausal women examining this outcome (520). Skeletal fracture outcomes were not reported in any studies. The effect of combination $\mathrm{LT}_{4} / \mathrm{LT}_{3}$ treatment compared to $\mathrm{LT}_{4}$ alone on resting heart rate was evaluated in 11 trials (384$387,517-520,522-524)$, and the effects were characterized as follows: (i) significantly increased heart rate in the combination therapy group compared to the $\mathrm{LT}_{4}$ groupone trial by Appelhof et al. (386), at the 5:1 $\mathrm{LT}_{4}$ to $\mathrm{LT}_{3}$ ratio dose of combination therapy; (ii) significantly reduced heart rate in the combination therapy group compared to the $\mathrm{LT}_{4}$ group-three trials, including those from Bunevicius et al. (387), Escobar-Morreale et al. (519), and Walsh et al. (385), respectively, or (iii) no significant difference between treatment groups in seven trials $(384,517,518$, 520,522-524). Too few of the combination therapy trials have assessed lipid and cardiovascular parameters to allow comments about benefits related to these outcomes.

Another relevant consideration is treatment preference of patients (see comment in recommendation 12 about use of preference measures as health outcomes). An important limitation in interpreting data on patient preference from randomized trials of combination therapy is a lack of validation and standardization of measurement of this construct, among trials. Among the five blinded, cross-over design trials comparing treatment preference $(385,387,517,519,521)$, combination $\mathrm{LT}_{4} / \mathrm{LT}_{3}$ treatment was preferred to $\mathrm{LT}_{4}$ in four trials $(387,517,519,521)$, whereas there was no significant difference in treatment preference in only one trial (385). It is important to note that the total number of patients who completed the studies in these four positive cross-over design preference trials was only $128(387,517,519,521)$, which is 
Table 8. Trials from Those Listed in Table 7

That Provide Information Regarding Mood and Fatigue Symptoms in the Trial Participants

\begin{tabular}{ll}
\hline Reference & \multicolumn{1}{c}{ Information about mood and fatigue } \\
\hline 519 & $\begin{array}{c}\text { Individuals with mental illness or affective } \\
\text { disorders were not allowed to participate } \\
\text { in this trial. } \\
\text { The participants in this trial were prescreened } \\
\text { for fatigue and mood, using validated } \\
\text { questionnaires (Piper Fatigue Scale } \\
\text { and General Health Questionnaire-30 } \\
\text { [GHQ-30]), respectively. The sampling } \\
\text { of participants was stratified according } \\
\text { to severity of fatigue level; however, } \\
\text { individuals with scores > } 45 \text { on the } \\
\text { GHQ-30 were excluded, due to an } \\
\text { increased probability of depression } \\
\text { (530,532,533). } \\
\text { This trial was conducted in individuals } \\
\text { with primary hypothyroidism who were } \\
\text { had evidence of depressive symptoms } \\
\text { at baseline (GHQ administered on two } \\
\text { occasions prior to randomization) (404,531). } \\
\text { Individuals with psychiatric disorders were } \\
\text { not allowed to participate in this trial. } \\
\text { Individuals with untreated major depression } \\
\text { were not allowed in this trial, but patients } \\
\text { on stable doses of antidepressants were } \\
\text { allowed if the depression treatment was } \\
\text { unlikely to change. }\end{array}$ \\
524 &
\end{tabular}

comparable to the 101 patients who completed follow-up in the trial in which combination treatment was not preferred (385). The mean serum TSH was significantly higher in the combination therapy group $(3.1 \mathrm{mIU} / \mathrm{L})$ compared to the $\mathrm{LT}_{4}$ group (mean $1.5 \mathrm{mIU} / \mathrm{L}$ ) in the negative cross-over design preference trial (385); in the cross-over trials where combination treatment was preferred, the mean serum TSH was not significantly different between groups in three studies $(387,517,521)$, but significantly higher in the combination $\mathrm{LT}_{4} / \mathrm{LT}_{3}$ group compared to the $\mathrm{LT}_{4}$ group in one study from Escobar-Morreale et al. (519) (mean TSH $2.5 \mathrm{mIU} / \mathrm{L}$ in the combination therapy group and $1.95 \mathrm{mIU} / \mathrm{L}$ in $\mathrm{LT}_{4}$ group). In blinded parallel design randomized trials evaluating patient preference or subjective patient evaluation of improved wellbeing on experimental treatment, combination treatment was preferred over $\mathrm{LT}_{4}$ alone in a study examining data from 130 individuals from Appelhof et al. (386), but not in a study examining data from 573 individuals from Saravanan et al. (522). The observed treatment preferences of $\mathrm{LT}_{4} / \mathrm{LT}_{3}$ combination therapy over $\mathrm{LT}_{4}$ treatment alone in the described studies cannot be reliably explained by any significant consistent benefit of combination treatment on measures of mood, quality of life, neurocognitive functioning, classic hypothyroid symptoms, weight, or TSH. Thus, the reason why $\mathrm{LT}_{4} / \mathrm{LT}_{3}$ combination therapy was preferred over $\mathrm{LT}_{4}$ alone by some patients is not well understood, and there is some question about validity of this measurement, given the lack of standardized evaluation methods for this construct among studies.

We also reviewed the results of recent systematic reviews or meta-analyses, comparing the effect of combination $\mathrm{LT}_{4} / \mathrm{LT}_{3}$ treatment compared to $\mathrm{LT}_{4}$ treatment alone $(389,525-527)$. For respective meta-analyses, the timing of review and number of trials included was as follows: (i) review by Grozinsky-Glasberg et al. (389) conducted in September 2005 in which data were pooled from 11 trials (including one unpublished study), (ii) a review by Joffe et al. (526) from 2007 in which data were pooled from nine trials (including one unpublished trial), and (iii) a review by Ma et al. (527) conducted in 2008 in which data were pooled from 10 trials [including potentially partially duplicated results of one trial (387)]. The authors of these meta-analyses reported that based on their pooled analyses, there was no significant benefit of combination $\mathrm{LT}_{4} / \mathrm{LT}_{3}$ treatment compared to $\mathrm{LT}_{4}$ treatment alone, on mood/health-related quality of life $(389,526,527)$, nor cognitive functioning $(389,527)$. Of note, statistical heterogeneity of some of these pooled analyses was noted $(389,527)$, and for some measures reported by Ma et al. (527), $\mathrm{LT}_{4}$ alone appeared to be superior to combination treatment. Pooled adverse event rates were not significantly different between treatment arms (389). In a meta-regression analysis, GrozinskyGlasberg et al. (389) reported no significant association among length of follow-up, percentage of athyreotic patients, or dose of $\mathrm{LT}_{3}$ and psychologic outcomes. In pooling data from four trials examining treatment preference, Joffe et al. (526) reported that combination treatment was significantly preferred over $\mathrm{LT}_{4}$ treatment alone. A systematic review, without metaanalysis, was also published by Escobar-Morreale et al. (525) in 2005, in which data from nine trials [including the 1970 trial from Smith et al. (528)], were reviewed. Escobar-Morreale et al. (525) also concluded that data on benefits of combination treatment on psychological outcomes were heterogeneous and not reproducible, and that the treatment preference for combination treatment was not explained based on results of psychologic or psychometric tests.

In conclusion, in reviewing data from 13 published RCTs (384-388,517-524) and four systematic reviews or meta-analyses of trials $(389,525-527)$, we found important unexplained clinical heterogeneity of studies in terms of therapeutic dose combinations utilized and important study results, such as the following: end of trial TSH, psychological status or health-related quality of life, neurocognitive functioning or behavior, and treatment preference. Measurements on thyroid-related symptom questionnaires generally did not appear to be significantly superior with $\mathrm{LT}_{4} / \mathrm{LT}_{3}$ combination therapy compared to $\mathrm{LT}_{4}$ alone. Serious adverse events did not appear to be reported at higher rates in combination treatment compared to $\mathrm{LT}_{4}$ alone; however, the majority of trials were limited by relatively short follow-up periods. The potential for adverse effects of long-term use of combination therapy, such as fractures or cardiovascular events, are not known. There is also a paucity of data collected on the use of combination therapy in men with primary hypothyroidism. Longer-term outcome research is generally needed in this field, given that TH replacement therapy is generally lifelong. Furthermore, RCTs incorporating the use of sustained release $\mathrm{T}_{3}$ preparations would be of interest. However, at the current time, based on a lack of clear consistent evidence of benefit of combination therapy using $\mathrm{LT}_{4} / \mathrm{LT}_{3}$ over established $\mathrm{LT}_{4}$ therapy and the lack of a proven reproducibly efficacious dose combination using currently available preparations, as well as the lack of long-term outcome data (on safety and efficacy) on the use of $\mathrm{LT}_{4} / \mathrm{LT}_{3}$ combination therapy, we 
believe that the routine use of $\mathrm{LT}_{4} / \mathrm{T}_{3}$ in treatment of primary hypothyroidism is not justified.

Published trials of combination therapy have not targeted patients who have relatively low serum $T_{3}$ values during monotherapy. In addition, the serum $\mathrm{T}_{3}$ values achieved during combination therapy have not been a specific endpoint of these trials. It is possible that future trials that include having both a relatively low serum $\mathrm{T}_{3}$ and a normal serum TSH concentration during monotherapy as entry criteria, in the absence of nonthyroidal illness, might have different outcomes from the trials conducted thus far. Future research addressing this subgroup of patients should be encouraged because this is currently an unproven approach. As outlined in the discussion of recommendation $9 \mathrm{~b}$, some of our task force members felt that, at the discretion of the practitioner, innovative therapy with $\mathrm{T}_{3}$ could be attempted. Such treatment would be solely used to attempt to enhance the well-being of an individual patient, but would not formally test a hypothesis, permit conclusions to be drawn, or contribute to knowledge that would generalizable for future patients.

Our view is generally in keeping with a recent joint statement from the AACE and the ATA, indicating that "the evidence does not support using L-thyroxine and Ltriiodiothyronine combinations to treat hypothyroidism" (3). However, the joint AACE/ATA hypothyroidism treatment guideline acknowledged that there are "still-unresolved issues raised by studies that reported some patients prefer and some patient subgroups may benefit from a combination of Lthyroxine and L-triiodothyronine" (3). In a recent clinical practice guideline, the ETA has also stated that "there is insufficient evidence that $\mathrm{LT}_{4}$ and $\mathrm{LT}_{3}$ combination therapy serves the hypothyroid patient better than $\mathrm{T}_{4}$ monotherapy" and that " $\mathrm{LT}_{4}$ remains the standard treatment for hypothyroidism" (5). However, the ETA has suggested that "LT 4 and $\mathrm{LT}_{3}$ combination therapy might be considered as an experimental approach in compliant $\mathrm{LT}_{4}$-treated hypothyroid patients who have persistent complaints despite serum TSH values in the reference range, provided they have previously been given support to deal with the chronic nature of their disease and associated auto-immune diseases have been ruled out." This document outlines methods for calculating $\mathrm{LT}_{4}$ and $\mathrm{LT}_{3}$ doses for physicians who are considering using a trial of combination therapy in their patients. A range of widely differing opinions about combination therapy has been offered since the publications of the AACE/ATA and ETA guidelines (534-539). However, both the AACE/ATA hypothyroidism guideline (3) and the ETA guideline (5) have strongly discouraged the use of $\mathrm{LT}_{4} / \mathrm{LT}_{3}$ combination therapy in pregnant women. We also agree that in pregnancy, where delivery of $T_{4}$ is thought to be crucial for fetal brain development (483), use of a treatment regimen containing $\mathrm{T}_{3}$, in which the $\mathrm{T}_{4}$ concentration may be relatively reduced, could be deleterious and should be avoided.

We would like to stress that if any experimental therapy (such as combination $\mathrm{T}_{4} / \mathrm{T}_{3}$ therapy) is considered in humans, it must meet professional and institutional ethical standards, as outlined in section 18 (ethical considerations in hypothyroidism research). We believe that high quality randomized controlled clinical trials are needed to prove if any specific subgroup of patients with primary hypothyroidism may specifically benefit from the use of $\mathrm{T}_{4} / \mathrm{T}_{3}$ combination ther- apy over the use of $\mathrm{T}_{4}$ alone. Such trials need to track relevant adverse effects, and ideally, examine long-term outcomes.

\section{3d. Should genetic characterization according to type 2 deiodinase gene polymorphism status be used to guide the use of combination synthetic levothyroxine and liothyronine therapy in hypothyroidism, in order to optimize biochemical and clinical outcomes?}

\section{- RECOMMENDATION}

Currently, genetic testing is not recommended as a guide to selecting therapy for three reasons. (i) Although there are data suggesting that specific polymorphisms of the type 2 deiodinase gene might be associated with therapeutic response to combination synthetic levothyroxine and liothyronine therapy, controlled confirmatory studies are needed. (ii) Currently genetic testing for these specific deiodinase polymorphisms is only available in the research setting. (iii) The small effect of the type 2 deiodinase gene variants identified so far that do affect thyroid hormone concentrations (see section 13a), suggests that other factors (e.g., yet unidentified genetic variants) may play a far greater role in determining an individual patient's thyroid hormone concentrations.

\section{Strong recommendation. Moderate quality evidence.}

\section{Discussion of the clinical literature}

We examined the medical literature to determine whether a therapeutic strategy of tailoring the use of combination $\mathrm{LT}_{4} /$ $\mathrm{LT}_{3}$ treatment, according to genetic characterization of the primary hypothyroid adult patient, improves health outcomes, as opposed to a strategy of routine $\mathrm{LT}_{4}$ treatment for all patients. We included three studies on secondary analyses $(390,540,541)$ stemming from two prospective trials comparing $\mathrm{LT}_{4} / \mathrm{LT}_{3}$ combination therapy to $\mathrm{LT}_{4}$ alone in primary hypothyroidism $(386,522)$ in our review. Such secondary analyses of trials, in which treatment allocation was not stratified a priori according to genetic characterization, should be considered hypothesis generating. In addition, given the modest effects that we now know these polymorphisms exert, this may limit their clinical relevance for the individual patient.

Using data from a RCT by Saravanan et al. (522), Panicker et al. (390) explored in a secondary analysis whether common genetic variants (polymorphisms) of three deiodinase genes (DIO1, DIO2, and DIO3), predicted baseline psychological morbidity, and response to combination $\mathrm{LT}_{4} / \mathrm{LT}_{3}$ treatment in 552 patients. Panicker et al. (390) reported that the presence of two SNPs in the DIO2 gene, DIO2-Thr92Ala (also known as rs225014) and rs225015, which are in strong linkage disequilibrium $\left(R^{2}=0.88\right)$, were respectively significantly associated with baseline measures of psychological well-being. Although 16 polymorphisms were tested, no multiple testing correction was applied because this study, despite being the largest study to date, was underpowered to detect all but very large differential gene-treatment effects. For this reason, the authors choose to report the $p$ values and associations, which should be considered suggestive, and qualified their findings by stating clearly that the results need replicating as a risk of type I statistical error exists (390). 
Moreover, Panicker et al. (390) reported a significant interaction between the less common variant of DIO2-Thr92Ala and combination $\mathrm{LT}_{4} / \mathrm{LT}_{3}$ treatment compared to $\mathrm{LT}_{4}$ treatment on the outcomes of psychological well-being, thyroid symptoms, and satisfaction, in the absence of any significant effect on circulating TH levels. In addition, there was no association between being homozygous for the $\mathrm{DIO} 2$ polymorphism and having a lower pre-intervention serum free $\mathrm{T}_{3}$ concentration than the remainder of the study population. Sixteen percent of the population in this study was homozygous for the polymorphism (390). In contrast, analyzing data from a RCT by Appelhof et al. (386) comparing $\mathrm{LT}_{4}$ monotherapy with $\mathrm{LT}_{4}$ and $\mathrm{LT}_{3}$ combination therapy in two ratios, the same group (540) found no significant association of DIO2-Thr92Ala and another DIO2 polymorphism DIO2258A/G (rs12885300, DIO2-ORFa-Gly3Asp) with measures of well-being, neurocognitive functioning, or treatment preference (data from 92 patients). Polymorphisms in DIO1, although associated with altered serum $T_{3}$ levels $(495,502)$, have not been associated with altered response to combination therapy (390).

In another secondary analysis of 92 patients from the same study by Appelhof et al. (386), van der Deure et al. (541) reported that the presence of two polymorphisms of OATP1Cl, a $\mathrm{T}_{4}$ transporter expressed in brain tissue, were significantly associated with measures of fatigue and depression, but did not explain differences in neurocognitive functioning nor preference for $\mathrm{LT}_{4} / \mathrm{LT}_{3}$ combination therapy in patients with primary hypothyroidism. An important limitation of these studies examining associations with SNPs was that respective treatment allocation to the combination $\mathrm{LT}_{4} / \mathrm{LT}_{3}$ treatment or $\mathrm{LT}_{4}$ alone, was not randomized with attention to polymorphism status, and thus the results should be considered hypothesis generating. In addition, the differences between the treatment groups were significant, but small, with considerable overlap between the groups. As a consequence, it is unlikely that genetic characterization of only these polymorphisms will result in superior health outcomes.

With whole exome and whole genome sequencing becoming ever more easily available, less common variants that may, in part, have larger effects are likely to be detected. Large RCTs are needed to know whether a strategy of tailoring the type of $\mathrm{TH}$ replacement therapy (i.e., $\mathrm{LT}_{4} / \mathrm{LT}_{3}$ or $\mathrm{LT}_{4}$ alone), according to genetic characterization, may result in superior health outcomes, compared to the currently recommended strategy of routine $\mathrm{LT}_{4}$ treatment for adults with primary hypothyroidism.

\section{Triiodothyronine Monotherapy for Hypothyroidism}

\section{Are there data regarding therapy with triiodothyronine alone, either as standard liothyronine or as sustained release triiodothyronine, that support the use of triiodothyronine therapy alone for the treatment of hypothyroidism?}

\section{- RECOMMENDATION}

Although short-term outcome data in hypothyroid patients suggest that thrice-daily synthetic liothyronine may be associated with beneficial effects on parameters such as weight and lipids, longer-term controlled clinical trials using a longer-acting form of triiodothyronine are needed before considering the endorsement of synthetic liothyronine therapy for routine clinical use.

\section{Strong recommendation. Moderate quality evidence.}

\section{Mechanistic background}

$T_{4}$, the main product of the thyroid gland, is a prohormone devoid of intrinsic activity, and the biological effects of TH are the result of the interaction between $T_{3}$ with its receptors (9). From this perspective $T_{4}$, and by extension its reservoir of hormone bound to serum carrier proteins, ensures a reserve of substrate for the deiodination to $T_{3}$ and offers a buffering against fluctuations in the circulating and tissue levels of $T_{3}$. On the other hand, since replacement therapy with $L T_{4}$ relies entirely on the deiodination step to provide adequate levels of serum and tissue $T_{3}$, a defect in this metabolic step would result in inadequate delivery of the active hormonal form. Direct therapy with $L T_{3}$, while having the advantage of avoiding the requirement for a deiodination step, could also have the theoretical disadvantage of not permitting regulated time-specific and tissue-specific production of $T_{3}$. With $L T_{4}$ therapy, D2-expressing tissues should maintain the required substrate for normal intracellular generation of $T_{3}$; in addition, the D2 pathway contributes to providing normal amounts of $T_{3}$ for most patients. With $T_{3}$ monotherapy, multiple daily dosing is required to sustain serum $T_{3}$ levels due to the shorter half-life of $L T_{3}$, compared with $L T_{4}$ (348). If normalization of serum TSH is the goal of $L T_{3}$ monotherapy, serum $T_{3}$ levels must be significantly higher, approximately double, than those seen during $L T_{4}$ monotherapy; whether this leads to relative hyperthyroidism in some tissues requires further study. The impact of $L T_{3}$ monotherapy on D2-expressing tissues other than the pituitary gland is also unknown. In rodents, normalization of serum TSH and hypothalamic TRH expression with $T_{3}$ monotherapy requires serum $T_{3}$ levels in the supranormal range (542).

\section{Discussion of the clinical literature}

Therapy with synthetic $\mathrm{LT}_{3}$ has the theoretical advantage of bypassing the $T_{4}$ to $T_{3}$ conversion step, which is obligatory in case of $\mathrm{LT}_{4}$ treatment, hence directly delivering the bioactive drug to the various organs/tissues that are the target of hormonal action. Conversely, a theoretical disadvantage of therapy with $\mathrm{LT}_{3}$ alone is represented by the lack of $\mathrm{T}_{4}$ as a reservoir of substrate; hence the circulating and tissue levels of $T_{3}$ are entirely dependent on the pharmacokinetic characteristics of the exogenously administered hormone replacement. The available data relative to the pharmacokinetics of $\mathrm{LT}_{3}$ are contradictory, indicating half-life values ranging from 6 to 22 hours $(36,543)$. The primary data were obtained from single-dose studies, and these studies were either performed by means of measurement of radioactive decay of radio-labeled $\mathrm{LT}_{3}$ in euthyroid volunteers (543) or by measurement of serum $\mathrm{T}_{3}$ in hypothyroid patients $(36,543)$. Since some studies have been performed in subjects with endogenous production of $\mathrm{TH}$ from the thyroid gland, it is possible that the re-uptake and metabolism of iodine in the thyroid caused a spurious increase in the half-life of the drug. Calculation of $\mathrm{LT}_{3}$ half-life in the blood may also be complicated by distribution from the blood into other 
compartments and redistribution back into the blood. However, an average half-life of 1 day has been quoted (543).

$\mathrm{LT}_{3}$ therapy started after $\mathrm{LT}_{4}$ discontinuation, usually administered at a dose of $25 \mu \mathrm{g}$ twice daily, has traditionally been used with the intent of ameliorating hypothyroid symptoms in situations in which induction of hypothyroidism was used as a means delivering therapy for thyroid cancer (544). However, with respect to the hypothyroidism induced in this situation, once-daily administration of $50 \mu \mathrm{g} \mathrm{LT}_{3}$ versus placebo did not result in a significantly different Billewicz score when documented 2 weeks after $\mathrm{LT}_{4}$ discontinuation, perhaps because $\mathrm{FT}_{4}$ levels were still sufficient at this time point. Use of $\mathrm{LT}_{3}$ delayed the attainment of a serum TSH greater than $30 \mathrm{mIU} / \mathrm{L}$ but did not alter the hypothyroid symptoms at the time of scanning (44). Thus $\mathrm{T}_{3}$ does not appear to have an advantage in this setting.

Although an extended-release formulation of $\mathrm{LT}_{3}$ has been shown in a single short-term study to reduce the peaks and troughs of $\mathrm{LT}_{3}$ administration that were seen over a 9-hour period after $\mathrm{T}_{3}$ administration (545), currently no formal pharmacokinetic study has been published. Presumably, once in the circulation as $T_{3}$, the persistence of $T_{3}$ is limited by its short half-life. Thus, even though a sustained release preparation may have reduced peaks and troughs, it may still need to be administered several times day. However, interestingly, a report of a sustained release profile for serum $\mathrm{T}_{3}$ levels after administration of a single dose of $\mathrm{T}_{3}$ sulfate to hypothyroid patients was recently published (546).

A recent double-blind randomized cross-over study demonstrated that $\mathrm{LT}_{3}$ can achieve an equivalent degree of serum TSH suppression as compared to $\mathrm{LT}_{4}$ at a ratio $(\mu \mathrm{g} / \mu \mathrm{g})$ of $1: 3$ when administered as a thrice-daily regimen (348). With this regimen the serum levels of $\mathrm{T}_{3}$ were significantly higher, albeit within the reference range, than the ones achieved with $\mathrm{LT}_{4}$ therapy both in the morning and throughout the day (347), while serum TSH remained stable throughout the 24 hours. In this study, no adverse events were recorded with the exception of an episode of generalized anxiety during the treatment with $\mathrm{LT}_{4}$; the two treatments were also equivalent with respect to cardiovascular function and glucose homeostasis. No differences were observed in quality-of-life endpoints, and patients were not able to distinguish which treatment arm they were assigned to (347). When treated with $\mathrm{LT}_{3}$, patients experienced an average weight loss of $2.1 \mathrm{~kg}$, a $13.3 \%$ decrease in LDL-cholesterol, and a $22.3 \%$ increase in SHBG, compared with $\mathrm{LT}_{4}$ therapy. These investigators subsequently reported performing TRH stimulation in these patients and noted that serum TSH responses were similar in the $\mathrm{LT}_{3}$ and $\mathrm{LT}_{4}$ groups, suggesting that possible metabolic differences were mediated peripherally and not at the pituitary gland level (373). Taken together, the data indicate that the substitution of $\mathrm{LT}_{3}$ for $\mathrm{LT}_{4}$ at equivalent doses (relative to the thyrotroph) provide an increased hormonal signal to the liver in the absence of obvious toxicity.

Although the results of the double-blind randomized crossover treat-to-target $\mathrm{LT}_{3}$ versus $\mathrm{LT}_{4}$ study (347) seem to indicate some superiority at least relative to weight and lipid profile, it is important to note that the experimental treatment was cumbersome and required multiple adjustments; indeed volunteers required an average of 180 days to achieve a target serum TSH on a stable dose of replacement therapy. Therefore, $\mathrm{LT}_{3}$ alone should not be advocated as a replacement therapy modality in the absence of an extended-release formulation able to provide stable serum levels of $\mathrm{T}_{3}$.

The $\mathrm{LT}_{3}$-only therapy appears more favorable for patients affected by dyslipidemia and obesity, but at the present time there is not sufficient evidence that this modality is superior to the standard $\mathrm{LT}_{4}$ therapy, particularly because of the risk of over- or underdosing, with the attendant risk of cardiac and skeletal toxicity, and because of the necessity of strict compliance to the regimen and the timing of the drug administration.

Presently no data are available on the long-term effects of $\mathrm{LT}_{3}$-only therapy, particularly with respect to bone and mineral metabolism and overall safety profile. Long-term administration studies on large populations are required to evaluate the safety of this treatment modality, and the sustainability of the weight and lipid profile changes. Currently there is no evidence that $\mathrm{LT}_{3}$-only therapy is cost-effective relative to the standard of care represented by $\mathrm{LT}_{4}$. Furthermore, one can predict that this therapeutic modality will require more monitoring and presumably will be associated with longer periods of undertreatment or overtreatment as compared to $\mathrm{LT}_{4}$ therapy.

Although not recommended, if used, the following common sense recommendations to monitoring might apply. $\mathrm{LT}_{3}$-only therapy should be monitored with measurements of fasting serum TSH levels before the administration of the dose, aiming to target serum TSH within the lower tertile of the reference range, if aiming for TSH values seen in a normal population (54). Although data to support this approach are not available, it would seem reasonable to measure serum $\mathrm{T}_{3}$ levels in the morning (trough) and 2 hours after the administration of the dose (peak), aiming to maintain the values within the reference range; serum $\mathrm{FT}_{4}$ assessment is of no value in this scenario.

At the present time there is insufficient evidence to suggest that $\mathrm{LT}_{3}$-only therapy is a safe and efficacious treatment of hypothyroidism in the general population, including in hypothyroid patients with obesity and dyslipidemia. This recommendation may change in the future should long-term, randomized, controlled trials of a sustained release formulation of $\mathrm{T}_{3}$ become available.

\section{Liothyronine Monotherapy in Euthyroid Patients}

\section{5a. Is there a role for the use of liothyronine to treat biochemically euthyroid patients with depression?}

\section{- RECOMMENDATION}

Although some uncontrolled and nonrandomized data exist concerning successful use of liothyronine in euthyroid patients with depression, larger, prospective randomized placebo-controlled studies are needed to more completely define the potential role of liothyronine in this condition, balancing the risks and benefits of therapy to measurable clinical outcomes.

\section{Weak recommendation. Low-quality evidence.}

\section{Discussion of the clinical literature}

Despite advances in our understanding of $\mathrm{T}_{3}$-mediated genomic as well as nongenomic actions at the cellular level 
(547), the role for $\mathrm{LT}_{3}$ in optimizing $\mathrm{TH}$ replacement therapy has not been clearly elucidated $(534,548-550)$. With increased recognition of the biologic importance of $\mathrm{T}_{3}$ at the cellular level, and the concerns that biochemical euthyroidism may not equate to tissue euthyroidism, there has been an increasing dialogue regarding whether $\mathrm{LT}_{3}$ therapy may be effective in reducing symptoms seen in euthyroid patients as well as in patients with depression, obesity, and cardiovascular disease.

The potential use of $\mathrm{LT}_{3}$ in the treatment of mood disorders has most commonly focused on one of two clinical strategies, either as an adjunct to help accelerate the onset of action of antidepressant therapy or as an augmentation to therapy in an effort to enhance the effect of treatment that has not achieved the intended clinical effect (551). Hypothalamic-pituitarythyroid axis abnormalities have been documented in depression, along with reduction in serotonin levels (552). The physiologic basis for the potential role of $\mathrm{LT}_{3}$ is based on observations of $\mathrm{T}_{3}$-induced increased concentrations of serotonin in the cerebral cortex of mice with increased serotoninmediated responses (553). In a meta-analysis by Aronson et al. (554) of patients with major depressive disorder, $\mathrm{LT}_{3}$ augmentation was associated with a twofold greater likelihood of response to tricyclic antidepressant (TCA) therapy in 292 patients with a previous history of treatment-resistant depression. Altshuler et al. (555) reported results of a metaanalysis of six studies examining the use of $\mathrm{LT}_{3}$ to accelerate the effect of TCA with five of six studies showing greater effect of combined therapy with $\mathrm{LT}_{3}$ over placebo, with the most pronounced impact found in studies that had larger cohorts of women. More recently, Cooper-Kazaz and Lerer (551) reviewed the literature on combined $\mathrm{LT}_{3}$ and serotonin re-uptake inhibitors (SSRIs). A total of eight studies were compared; five RCTs, of which three were enhancement studies and two were augmentation studies, and three additional open augmentation studies in which $\mathrm{LT}_{3}$ was added after failure of SSRI-only therapy. Of the three enhancement studies, one showed weak support for acceleration by $\mathrm{LT}_{3}$ of the therapeutic efficacy of SSRIs, and in two, no acceleration was found. In the five augmentation studies, including the STAR*D study comparing $\mathrm{LT}_{3}$ augmentation to lithium augmentation (556), the use of $\mathrm{LT}_{3}$ augmentation was associated with an overall remission rate between $25 \%$ and $36 \%$. For all studies combined there were very few side effects associated with the addition of $\mathrm{LT}_{3}$ up to $50 \mu \mathrm{g} / \mathrm{d}$ (551). The authors conclude that while there is evidence to support the clinical efficacy of $\mathrm{LT}_{3}$ for the enhancement or augmentation of SSRI therapy, the data are not conclusive and further controlled studies employing uniform study design are needed to determine the appropriate dose, the timing of $\mathrm{LT}_{3}$ initiation, and length of treatment (551). The practice guideline of the American Psychiatric Association describes the use of $\mathrm{LT}_{3}$ in its document about treatment of major depression, but does not have a specific recommendation addressing its use (557).

If $\mathrm{LT}_{3}$ therapy is in fact beneficial, it is possible that this may be due to pharmacological rather than physiological effects of $T_{3}$. Nevertheless, it may occur that a psychiatrist is using adjunctive oral $\mathrm{LT}_{3}$ therapy to treat a psychiatric disorder and requests an endocrinologist's opinion how to manage the patient. It would seem appropriate to monitor the patient clinically with a history and physical examination.
Serum $\mathrm{FT}_{4}, \mathrm{~T}_{3}$, and TSH levels should also be monitored, recognizing that the $\mathrm{T}_{3}$ levels, and perhaps the TSH concentrations, might vary throughout the day. A clinical recommendation then can be given based on these parameters.

\section{5b. Is there a role for the use of liothyronine to treat biochemically euthyroid patients with obesity?}

\section{- RECOMMENDATION}

We recommend against the use of synthetic liothyronine therapy in treating euthyroid patients with obesity, due to a lack of controlled data proving treatment efficacy for this indication.

\section{Strong recommendation. Low-quality evidence.}

\section{Discussion of the clinical literature}

$\mathrm{LT}_{3}$ has also been considered as a potential therapy to prevent the cardiovascular morbidity and mortality associated with obesity. Efforts to use $\mathrm{LT}_{3}$ are based on studies showing decreased serum $T_{3}$ with subsequent decreased basal metabolic rate in obese subjects placed on calorierestricted diets $(447,558)$. In hypothyroid patients, when $\mathrm{LT}_{3}$ is administered thrice daily, dosed to achieve a target TSH of $0.5-1.5 \mathrm{mIU} / \mathrm{L}, \mathrm{LT}_{3}$ treatment is associated with decreased body weight, with an average weight loss of $-1.8 \pm 1.9 \mathrm{~kg}$, as well as reduced total cholesterol, LDL, non-HDL, and apolipoprotein $\mathrm{B}$ when compared to $\mathrm{LT}_{4}$ therapy in the same individual (347). There have been multiple studies on the potential efficacy of $\mathrm{LT}_{3}$ in euthyroid obese subjects, summarized in a systematic review by Kaptein et al. (447). In 13 of 14 studies, $\mathrm{LT}_{3}$ was the exclusive therapy, with $\mathrm{LT}_{4} / \mathrm{LT}_{3}$ used in the additional study. Unfortunately, there was wide variation in study design with no clear association between $\mathrm{LT}_{3}$ therapy and weight loss, protein breakdown, or metabolic rate. In addition, none of the studies were designed to determine one of the most important questions; that is, whether $\mathrm{LT}_{3}$ therapy during caloric deprivation enhances fat loss without loss of muscle mass or strength. In addition, there was evidence showing $\mathrm{LT}_{3}$ therapy induced subclinical hyperthyroidism based on lower $\mathrm{FT}_{4}$ and TSH levels. No study performed TRH stimulation to more fully evaluate the impact of the $\mathrm{LT}_{3}$ dose regimen on hypothalamic-pituitary function (447).

\section{Compounded Thyroid Hormones}

\section{What is the recommendation regarding therapy with compounded thyroid hormones (either levothyroxine or liothyronine) for treatment of hypothyroidism based on current evidence?}

\section{- RECOMMENDATION}

We recommend against the routine use of compounded thyroid hormones due to concerns about safety and potency and due to the lack of data proving superiority to standard thyroid hormone preparations. However, in the case of suspected allergy to an excipient of standard thyroid hormone preparations that cannot be avoided with a change in brand or dose formulation, including a trial of levothyroxine gel capsules, it may be reasonable to 
consider use of compounded products, although a controlled study of this approach has not been published.

\section{Strong recommendation. Low-quality evidence}

\section{Discussion of the clinical literature}

Compounded medications are those prepared by a specialized pharmacy that produces the drug with specific individual patient needs in mind (in response to a licensed physicians' prescription). The process usually involves modifying or altering the components of the medication or its excipients in a specific manner. There are specialized pharmacies and pharmacists that are capable of preparing compounded medications. Theoretical advantages of compounded medications include the ability to change the form of the medication (e.g., from tablet to liquid or transdermal preparation), specific excipients or dyes can be included or excluded (e.g., lactose can be excluded if a patient is lactose intolerant, erythrosine [red dye] can be excluded if the patient is allergic), and various flavors can be added to make the medication more palatable. Specific doses of medication that may not be commercially available may also be prepared. On the other hand, there are potential concerning issues regarding compounded medications. There are no specific guidelines or regulations governing the compounding of every medication, and the experience and consistency regarding the active ingredient(s) may vary over time and between different pharmacies and different preparations. Furthermore, compounded medications may be more expensive than commercially prepared formulations.

The Pharmacy Compounding Accreditation Board has been developed to set national standards for compounding pharmacies. The FDA does not specifically regulate every medication that is prepared individually by compounding, nor does it regulate the specific pharmacy or pharmacists. The FDA does, however, oversee the ingredients used and the facilities in which they are prepared. It was noted in 2007 that the FDA considers compounded medications as "new drugs" and, as such, they do not regulate their safety or efficacy and also do not allow interstate transportation of these compounded medications unless approval is given in a specific instance (559). The FDA does recognize the critical role of individual states in supervising and regulating pharmacists and pharmacies that perform compounding of medications. FDA-cited concerns regarding compounded medications include the consistency of the skill of compounding pharmacists and the reliability of their products. The general conceptual importance of compounded medications for individual, specific patient usage, however, was noted. Thus, it is possible that these pharmacies could serve a role if bioequivalence and stability of hormonal content could be assured. It should also be noted that a recent fungal meningitis outbreak in Massachusetts was apparently linked to an intravenous compounded medication. This tragedy has led to comments and criticism of the present regulations governing compounding pharmacies. The FDA has suggested there be updated regulations governing compounding pharmacies with governmental oversight of the preparation, labeling, and administration of compounded medications, but still within a framework of maintaining the potential advantages of compounding pharmacies (560).
$\mathrm{T}_{4}, \mathrm{~T}_{3}$, thyroid extract, and combinations of these preparations have each been compounded. However, there are very few published studies regarding use of compounded THs for treatment of hypothyroidism that meet scientific criteria for rigorous peer review. One article published by a specific pharmacy owner lists suggested formulae for achieving a 11:1 ratio of $\mathrm{T}_{4}: \mathrm{T}_{3}$ in the circulation and describes preparation, but does not provide any details about outcomes of therapy with the compounded product (561). There are also no published studies that compare compounded preparations with FDAapproved preparations. Boulton et al. (562) assessed the stability of $\mathrm{LT}_{4}$ in compounded oral liquid doses prepared from commercially available tablets. They examined stability over time (up to 90 days) and under various temperature conditions. The compounded preparations had significant degradation in each of the circumstances noted, but the compounded tablet preparations without added preservative retained more than $90 \%$ of their original concentration for 8 days, although at day 14 none of the formulations retained more than $90 \%$ potency. Thus, in order to ensure maintenance of potency, fresh supplies may need to be obtained from the compounding pharmacy at frequent intervals, with associated inconvenience to the patient. A separate issue is related to the standardization and bioequivalence of compounded $\mathrm{T}_{4}$ and $\mathrm{T}_{3}$ obtained from a given pharmacy over time or between pharmacies. There are no peer-reviewed studies assessing these issues, thus quality control at the multiple pharmacies in existence is a concern. Errors in TH compounding have occurred and have resulted in severe thyrotoxicosis (563).

Unfortunately placebo-controlled comparative studies of compounded THs with their FDA-approved counterparts may not be helpful for guiding their use because the compounding arm of such a trial would be specific only to the particular pharmacist and compounding pharmacy. In other words, in light of the heterogeneity introduced by compounding pharmacists, if equivalence is demonstrated this would not be generalizable to other pharmacies. Therefore, randomized controlled clinical trials will be useful only when a uniform standard of regulation is instituted. When such trials are performed, in vitro studies should also determine the amount of $\mathrm{TH}$ in each preparation.

A general statement regarding the comparison of costs to the patient for a compounded $\mathrm{TH}$ and a commercially available product cannot be made. Patient costs for an individual compounded agent depends on multiple factors, to include the individual compounding pharmacy, the specific medication, the constituents of the compounding formulation, and insurance issues.

Until all the concerns above are addressed, potential use of compounding pharmacies should only be considered if there is no alternative, such as allergy to an excipient. However, it is theoretically possible that this indication may be obviated by the availability of $\mathrm{LT}_{4}$ soft gel capsules. The use of a nonexcipient gel capsule would be preferable to the use of compounded $\mathrm{LT}_{4}$.

\section{Nutraceuticals}

17. Is there a role for the use of dietary supplementation, nutraceuticals, and over-the-counter products in either hypothyroid or euthyroid individuals? 


\section{- RECOMMENDATION}

We recommend against the use of dietary supplements, nutraceuticals, or other over-the-counter products either in euthyroid individuals or as a means of treating hypothyroidism. We particularly caution against the use of pharmacologic doses of iodine because of the risk of thyrotoxicosis and hypothyroidism in those with intact thyroid glands susceptible to becoming further dysregulated because of underlying thyroid pathology.

\section{Strong recommendation. Low-quality evidence.}

\section{Discussion of the clinical literature}

The FDA 1994 Dietary Supplement Health and Education Act expanded the definition of dietary supplements to include any nontobacco product that is intended to supplement the diet and contains at least one of the following ingredients: a mineral, vitamin, herb or other botanical, an amino acid, or any dietary substance in humans utilized to supplement the diet by increasing the total daily intake, or a concentrate, metabolite, constituent, extract, or combinations of these ingredients.

Nutraceuticals is a term formulated to reflect its "nutrition" origin and "pharmaceutical" action, but it does not have a "regulatory definition." Nutraceuticals are dietary supplements that "contain a concentrated form of a presumed bioactive substance originally derived from a food, but now present in a non-food matrix, and used to enhance health in dosages exceeding those obtainable from normal foods" (564). Use of such products, including vitamin preparations and herbal supplements, is common (565). Approximately $20 \%$ of the subset of the hypothyroid population who are being treated for thyroid cancer use such supplements (566).

The AACE has published an excellent summary with guideline recommendations for dietary supplements and nutraceuticals (567), with some updated information provided in the recent Guidelines for Hypothyroidism in Adults (3). In general, the government mandates three possible types of claims of dietary supplement labels: (i) nutrient content (such as "high in calcium"); (ii) structure-function or nutrition support (for example, "vitamin C prevents scurvy" or "calcium builds strong bones"), and (iii) disease claims. The "disease claims" alone requires FDA authorization after a thorough scientific evaluation of relevant studies. All other products must include the following statement: "This statement has not been evaluated by the FDA. This product is not intended to diagnose, treat, cure, mitigate, or prevent any disease." Interested individuals should refer to the original comprehensive AACE document (567). Certain dietary supplements or nutraceuticals are purported to have the capacity to enhance thyroid function. These agents or compounds can be allocated to one of several categories: iodinecontaining substances, tyrosine-containing substances, and TH extracts or analogs.

Iodine-containing substances (e.g., kelp) can, indeed, have multiple actions on the thyroid gland, as illustrated in a recent series of case reports (568). These effects on the thyroid gland can have significant clinical consequences. Excess iodine may precipitate thyroid dysfunction, particularly in patients with underlying thyroid autonomy, Hashimoto's thyroiditis, or multinodular thyroid glands. Large amounts of iodine (e.g., SSKI or Lugol's solution) can be used to decrease thyroid gland $\mathrm{T}_{4}$ and $\mathrm{T}_{3}$ secretion and is used in the treatment of hyperthyroid patients (569). However, there can be escape from thyroidal inhibition of organification (i.e., escape from the Wolff-Chaikoff effect) (570), and exacerbation of hyperthyroidism can occur, especially in hyperthyroid patients treated with iodine solutions for at least several weeks or who are not receiving anti-thyroid agents concurrently. These studies demonstrate various and multiple effects of iodine supplementation on thyroid function including the inducement of hyperthyroidism, hypothyroidism, and thyroiditis. There is no doubt, of course, that a minimal amount of iodine (about $150 \mu \mathrm{g}$ daily) is required for normal thyroid function in euthyroid nonpregnant individuals, but when iodine supplementation is used by otherwise normal individuals to enhance thyroid function it is generally used in much larger doses, with the possibility of causing adverse effects. However, there is little evidence that iodine supplementation in pharmacologic amounts can beneficially improve thyroid function in euthyroid individuals. As a result, the AACE guidelines note that "No data support the role of iodine in enhancing thyroid function (patient advised to discontinue use)."

Tyrosine is an amino acid that that forms the basis for synthesis of $\mathrm{T}_{4}$ and $\mathrm{T}_{3}$, and it has been speculated that giving tyrosine supplementation to euthyroid individuals would improve thyroid gland function. Nonetheless, as noted in the AACE guidelines "No published data, however, support the claim that ingestion of tyrosine increases the production of TH (patient advised to discontinue use)." Commercially available supplements sold for "thyroid health" or "thyroid support" may also contain measurable amounts of $\mathrm{T}_{4}$ and $\mathrm{T}_{3}$, potentially exposing the public to iatrogenic thyrotoxicosis (571).

The AACE guidelines note that multiple substances have been utilized as dietary supplements for thyroid conditions. They list the following substances as being utilized for "functional hypothyroidism" in biochemically normal individuals: carnosic acid, Commiphora molmol (myrrh), desiccated animal thyroid extract, linoleic acid, omega-3 fatty acids, organic iodide, selenium, methionine, 3,5,3'-triiodothyroacetic acid or tiratricol (TRIAC), tyrosine, vitamin A, vitamin D, vitamin E, Withania somnifera, and zinc glycinate. They also note possible interactions between $\mathrm{TH}$ absorption or action with several compounds to include bugleweed, red yeast, kelp, calcium, iron, and bone meal. Soy protein has been studied and may interfere with $\mathrm{LT}_{4}$ absorption. Sathyapalan et al. (572) performed a double-blind cross-over study and demonstrated that there was an increased risk of developing $\mathrm{OH}$ in patients who originally had $\mathrm{SCH}$, when they received a vegetarian diet (containing $30 \mathrm{~g}$ soy protein and $16 \mathrm{mg}$ phytoestrogen) as compared to a Western diet (containing $30 \mathrm{~g}$ soy protein and $2 \mathrm{mg}$ phytoestrogens).

The use of various TH analogs that can be purchased over the internet to enhance thyroid function is a very controversial area. TRIAC is a naturally occurring metabolite of $\mathrm{LT}_{4}$ that has significant metabolic action. It can bind to the $\mathrm{T}_{3}$ receptor and it can decrease $\mathrm{TSH}$ secretion. It may have a role in managing cases of resistance to $\mathrm{TH}$ (573). However, TRIAC administration in pharmacologic doses can cause toxicity like that caused by TH (574-578). Although TRIAC has effects similar to $\mathrm{TH}$ (in selected circumstances), there is 
a lack of evidence it is beneficial when administered to euthyroid individuals and there is also a lack of evidence that it more beneficial than $\mathrm{LT}_{4}$ when administered to hypothyroid subjects (579). 3,5-Diiodothyronine is advertised on the internet as an agent for weight loss and enhancement of cardiovascular training. However, reports of its safety and efficacy for these purposes could not be identified, other than in a single short-term study in two patients (580). Given the lack of substantive available evidence, the use of a thyroid analog to enhance thyroid activity is not recommended.

Strong recommendation against nutraceuticals is provided despite low-quality evidence, because there are potential risks but few proven benefits.

\section{Ethical Considerations in Hypothyroidism Research}

\section{What are the research ethics issues involved in evaluating or designing clinical trials for the treatment of hypothyroidism?}

\section{- RECOMMENDATION}

There should be recognition that there are not enough data to resolve clinical disagreement among thyroid experts (called "clinical equipoise") regarding treatment for hypothyroidism. Clinical equipoise is disturbed only by the results of well-designed randomized controlled trials that have the statistical power to settle the question of efficacy between monotherapy and combination therapy, or other forms of therapy.

\section{Ungraded}

\section{Discussion of the clinical research literature}

In evaluating published research and original studies of $\mathrm{TH}$ replacement, only those studies demonstrating ethically sound design and responsible conduct of research (RCR), in which there was study oversight and Institutional Review Board approval, should be considered. Well-designed small studies without the statistical power to resolve clinical disagreement, or disturb "clinical equipoise" (see further), cannot be considered to be a high level of evidence of a null effect or superior or inferior efficacy.

The fact that a study is published does not mean that it meets criteria for RCR. A well-executed meta-analysis of high-quality RCTs, with no clinically or statistically significant heterogeneity of pooled results, could be used to influence clinical practice. However, narrative review articles, editorials, and opinion letters are insufficient to disturb clinical equipoise. There are fundamental challenges with editorship and reviewership, which can influence what types of studies are published (581). Although most industryfunded studies incorporate appropriate design and clear declaration of conflict, such studies can also be problematic. Studies funded by a drug manufacturer showing that the drug is effective or superior, need to be very carefully evaluated, as the opportunity for biased results is clear. In this respect, perceived conflicts of interest are treated the same as overt conflicts of interest.

Clinical equipoise in TH therapy. The concept of "clinical equipoise" was introduced in 1987 by research ethicist Benjamin Freedman (582). A state of clinical equipoise exists when a "community of experts" responsible for setting the standards of care are uncertain as to whether Therapy A is better than Therapy B. Thus, clinical equipoise provides the ethical basis for conducting a RCT of the two therapies in order to resolve the question about which therapy is superior. In current thyroidology, clinical equipoise exists about the issue of $\mathrm{LT}_{4} / \mathrm{LT}_{3}$ combination therapy for hypothyroidism as described in the literature review for recommendations $13 \mathrm{~b}$ and $13 \mathrm{c}$ and Table 7 . In this case, clinical equipoise may be considered to exist due to conflicting findings of trials in the literature and heterogeneity in the dose and frequency of the treatment utilized in the existing studies. There is also some uncertainty about which, if any, patients may benefit from combination therapy.

A critical question that must be addressed is: Who comprises the community of experts? There are some practitioners who feel that their own clinical observations in practice "count" as evidence and form the basis for "innovative therapies." Such practitioners may widely disseminate their therapeutic opinions to patients directly through published trade books or through the Internet. What separates a valid community expert from an invalid community expert is scientific integrity, in which there is honest clinical disagreement. However, practitioners who intentionally misuse their professional training to profiteer or deceive patients or misrepresent themselves as "thyroid experts" or thyroidologists when they can demonstrate no objective training in endocrinology are not considered ethical actors who are part of a serious clinical community with beneficent intentions. Clinical investigators who fail to meet basic standards in ethical conduct or scientific integrity must also be excluded from the community of experts. An example would be publishing results of new therapies without evidence of RCR. As for critics of TSH testing and proponents of antiquated diagnostic tools such as basal body temperature readings or other therapies, some (583) must be viewed as ethical actors within a history of medicine context if they were practicing in an era prior to the development of TSH testing as a standard of care. In this context, practitioners who questioned what would have been a "new" laboratory test in the 1960s and 1970s were actually practicing evidence-based medicine and were cautious about adopting a new standard of care until it proved to be a superior diagnostic tool. However, any practitioner who currently promotes antiquated ideas and judges them as "current" or still relevant misrepresents medical history. This would be similar to a diabetologist promoting the use of antiquated therapies for diabetes and recommending a patient with type 1 diabetes go on the special diets promoted in the early 20th century instead of taking insulin.

Resolving clinical equipoise: RCT design. The need to resolve collective professional uncertainty, or "community of experts" uncertainty, over efficacy and safety of Therapy A (combination therapy) versus Therapy B (standard of care/ monotherapy) is critically needed for patient care. However, we could not find sufficient consistent data from studies of long-term therapy to resolve these issues.

The community of experts in $\mathrm{TH}$ therapy remain divided over $\mathrm{T}_{4}$ monotherapy and $\mathrm{LT}_{4} / \mathrm{LT}_{3}$ combination therapy and even formulations of $\mathrm{T}_{4}$. This is creating problems in patient 
care, patient trust, and informed consent. The thyroid clinical community has an ethical obligation to disturb clinical equipoise regarding $\mathrm{TH}$ therapy in order to set a consistent standard of care that does not lead patients to question beneficent care. A robust RCT (power $=80 \%$ ) has yet to be conducted that recruits from a representative population of hypothyroid patients worldwide; tests therapies at dosages that cannot be disputed; accounts for regional disparities; discloses no potential conflicts of interest; and continues long enough for results to be replicated and to "resolve the dispute among clinicians" (582).

Design Criteria, in addition to incorporating clinically meaningful endpoints, ought to include the following (582):

- Who is enrolled? Clinical equipoise requires a representative sample of the target clinical population. One must be able to generalize the results to the conditions of clinical practice across health care systems and countries; narrow trials designed to resolve some theoretical question may render it useless for influencing clinical decisions, even if successfully completed. For example, studies performed in white males may not be applicable to women or minorities. There may be dosing discrepancies based on the pharmacogenetics of groups typically excluded from clinical trials.

- Sample size: If the sample is too small, it's unlikely to answer the question. A robust RCT (power $=80 \%$ ) would be likely to disturb equipoise even if the RCT result was "negative."

- What gets tested? Clinical equipoise requires honest, professional disagreement as to the preferred treatment: standard of care (monotherapy) versus new treatment (combination therapy) at dosages that are chosen based upon strong preliminary "proof-of-concept" data, and using doses and formulations that would not be contested by its proponents (584).

- When will the study be complete? The RCT ought to continue until sufficient evidence has been gathered to "resolve the dispute among clinicians" (582). This includes replication of results.

- Conflicts of interest: real or perceived. Studies receiving funding from a company with a financial interest in the study outcome may not disturb clinical equipoise due to perceived bias. Clinicians may also wish to be aware of the funding received from pharmaceutical companies by journals in which studies are published.

$N$-of-1 trials. $\quad N$-of-1 trials have been referenced in other reviews on this topic (5). In the few meta-analyses that have reviewed $N$-of- 1 trials (585), few even meet the criteria for an institutional review board (IRB)-approved study; most fall into the category of innovative therapy, which in the $\mathrm{TH}$ context is sometimes called "individualized" combination therapy. Innovative therapy refers to a nonstandard or even experimental therapy designed to optimize beneficent care for one individual patient and is not intended for generalizable knowledge. Such studies may also report "measurable effects" in individual patients that have no clinical significance. $N$-of- 1 trials may be useful for "enhancing therapeutic precision" or evaluating individual treatment effects for a patient (585). In order to be generalizable, a valid $N$-of- 1 trial is a well-designed, IRB-approved multiple cross-over study conducted with informed consent in a single individual as a tool to estimate the heterogeneity of treatment effects in a population. To date, no $N$-of- 1 studies in thyroidology meet this criteria; all of the $N$-of- 1 trials evaluated in the thyroid literature meet the standard of innovative therapy. Innovative therapies do not require IRB approval if data are not being collected for the purpose of generalizable knowledge and publication, and they are ethically permissible. Investigators who are using innovative therapies, in contrast to a situation in which an $\mathrm{N}$-of-1 trial is being conducted, are not truly engaged in research. Thus, any reports of innovative therapies cannot be used to validate or refute evidence, nor does innovative therapy resolve clinical equipoise.

\section{Wilson's Syndrome}

\section{Is there evidence for the existence of "Wilson's temperature syndrome" and a rationale for use of escalating doses of triiodothyronine?}

\section{- RECOMMENDATION}

There are no credible scientific data to support the existence of "Wilson's syndrome" and we recommend against the use of triiodothyronine escalation therapy for this indication, due to a lack of proven treatment benefit and safety concerns relating to the risk of thyrotoxicosis.

\section{Strong recommendation. Low-quality evidence.}

\section{Discussion of the clinical literature}

Wilson's syndrome or Wilson's temperature syndrome is described as having the hallmarks of fatigue, anxiety, depression, headaches, insomnia, and muscle aches, with a low body temperature being the feature that distinguishes it from chronic fatigue syndrome (586-588). The ATA statement on "Wilson's Syndrome" was updated on May 24, 2005 (589). The summary statement is as follows:

The American Thyroid Association has found no scientific evidence supporting the existence of "Wilson's syndrome." The theory proposed to explain this condition is at odds with established facts about thyroid hormone. Diagnostic criteria for "Wilson's syndrome" are imprecise and could lead to misdiagnosis of many other conditions. The $T_{3}$ therapy advocated for “Wilson's syndrome" has never been evaluated objectively in a properly designed scientific study. Furthermore, administration of $T_{3}$ can produce abnormally high concentrations of $T_{3}$ in the blood, subjecting patients to new symptoms and potentially harmful effects on the heart and bones.

The ATA supports efforts to learn more about the causes of somatic symptoms that affect many individuals, to test rigorously the idea that some as yet unidentified abnormality in thyroid hormone action might account for even a small subset of these symptoms, and to pursue properly designed clinical trials to assess the effectiveness of lifestyle, dietary, and pharmacological treatments for these common ailments. However, unsupported claims, such as those made for "Wilson's syndrome," do nothing to further these aims.

The task force reviewed this statement and performed a current literature review. As part of this review, the task force reviewed the Wilson's Syndrome Doctor's Manual (587). In addition, the posting from Wikipedia, the free encyclopedia, 
was also reviewed to determine what information the public has access to (590). The task force believes that the prior ATA statement accurately reflects our current state of knowledge. The protocol recommended by Dr. Wilson on his website remains of great concern, given that protocol describes escalating a dose of compounded $\mathrm{T}_{3}$ from $7.5 \mu \mathrm{g}$ twice daily by $15 \mu \mathrm{g}$ increments. A patient taking $7.5 \mu \mathrm{g} \mathrm{T}_{3}$ twice a day and increasing the dose by $15 \mu \mathrm{g}$ every day could potentially suffer serious iatrogenic $\mathrm{T}_{3}$ thyrotoxicosis. For example, see the section "patient death and medical license suspension" from the Wikipedia article on Wilson's syndrome.

A review article published since the 2005 ATA statement was identified (586). However, this article provided only the results of uncontrolled trials in support of $\mathrm{T}_{3}$ therapy, including an in-house trial of $\mathrm{T}_{3}$ therapy for chronic fatigue syndrome. It also quoted an uncontrolled trial of $\mathrm{T}_{3}$ therapy in individuals with persistent symptoms while taking $\mathrm{LT}_{4}$ performed prior to the availability of TSH monitoring (591). See also recommendation 16 for a discussion of compounded THs and recommendation 18 regarding the ethics of trials for the therapy of hypothyroidism. An ongoing dialogue with patients affected by the symptoms outlined on the Wilson's temperature website is essential. To quote from an article by Dr. Weetman (250), "communication lies at the heart of managing patients whose health problems cannot be explained and the focus should be on the patient's concerns, the relief of symptoms and the avoidance of alienation."'

\section{SECTION III. HOSPITALIZED PATIENTS}

Levothyroxine in Hospitalized Patients

\section{0a. In hospitalized but not critically ill patients with known pre-existing hypothyroidism, should levothyroxine therapy be re-evaluated based on an elevated serum thyrotropin measurement?}

\section{- RECOMMENDATION}

In hospitalized patients with a pre-established diagnosis of hypothyroidism who are found to have an elevated thyrotropin measurement, consideration should be given to institution or adjustment of levothyroxine replacement. Factors such as the degree of clinical and biochemical hypothyroidism, active comorbidities, and details of administration of levothyroxine (e.g., dosage, timing, and other factors impacting absorption) are relevant considerations in this situation.

Strong recommendation. Low-quality evidence.

20b. In hospitalized but not critically ill patients in whom levothyroxine replacement is instituted or increased, should the therapeutic goal be normalization of serum thyrotropin?

\section{- RECOMMENDATION}

The therapeutic goal of levothyroxine replacement in noncritically ill patients is long-term normalization of serum thyrotropin when steady-state thyroid hormone levels are achieved. We do not recommend titrating the levothyroxine dose to serum free thyroxine levels unless serum thyrotropin cannot be relied upon (e.g., following pituitary surgery).

Strong recommendation. Low-quality evidence.

\section{0c. In hospitalized but not critically ill patients treated with levothyroxine replacement what formulation and route of administration are recommended?}

\section{- RECOMMENDATION}

For hospitalized but not critically ill patients, oral levothyroxine treatment is recommended. If this is not feasible, other enteral routes can be used. However, if there are concerns about significant malabsorption or there are other clinical reasons why a patient cannot be given enteral levothyroxine, intravenous levothyroxine may be administered, until enteral absorption improves. If using intravenous levothyroxine, the equivalent intravenous dose is approximately $75 \%$, assuming the enteral levothyroxine dose had achieved euthyroidism.

\section{Weak recommendation. Low-quality evidence.}

\section{0d. In hospitalized but not critically ill patients about to be treated with levothyroxine should the possibility of adrenal insufficiency be excluded?}

\section{- RECOMMENDATION}

For hospitalized but not critically ill patients who are about to be treated with levothyroxine, the possibility of adrenal insufficiency should be considered. If there is sufficient clinical or biochemical evidence to consider this diagnosis, adrenal insufficiency should be ruled out or empiric treatment should be provided.

\section{Strong recommendation. Low-quality evidence.}

\section{Discussion of the clinical literature}

When a patient with pre-existing hypothyroidism is admitted to the hospital, it is not unusual to find that TSH levels are abnormal. The clinician must carefully investigate whether factors prior to the admission and/or factors relating to the admission itself are complicating the treatment of hypothyroidism; for example, omission of doses of $\mathrm{LT}_{4}$ or administration of medications that interfere with its absorption. When the patient is not critically ill, this process is usually straightforward. Best practice in this case is based on logic and observational studies or cases series, rather than clinical trials.

The situation is considerably more complicated if a patient with pre-existing hypothyroidism develops a separate critical illness. The nonthyroidal illness syndrome (NTIS) (also known as the sick euthyroid syndrome or the low $\mathrm{T}_{3}$ syndrome) occurs when patients with normal thyroid function become critically ill; in this setting, a progressive depression of the thyroid axis occurs such that circulating $\mathrm{T}_{3}$ decreases, followed by declines in serum TSH and/or $\mathrm{T}_{4}$, and then declines in $\mathrm{FT}_{4}$ in some sicker patients $(592,593)$. In current practice, these patients are not treated with $\mathrm{TH}$ replacement (see recommendation 22 regarding NTIS).

In contrast, patients with established hypothyroidism are maintained on TH when they become critically ill, even though they too may exhibit the physiologic changes of NTIS. This is logical, since untreated hypothyroidism itself can lead to critical illness in the form of myxedema coma. A rise in TSH during the worsening stages of critical illness is typically indicative of $\mathrm{TH}$ deficiency, and $\mathrm{TH}$ replacement is thus 
instituted or increased until TSH normalizes. Interpretation of thyroid function tests is more complicated in the intensive care unit (ICU) setting because of medication effects on the hypothalamic-pituitary axis. For example, dopamine and dobutamine may lower serum TSH, such that some patients with primary hypothyroidism could be under-replaced if following a TSH-dependent dosing scheme. $\mathrm{LT}_{4}$ may need to be given intravenously in the ICU setting since malabsorption can occur not only as a result of gastrointestinal disease, but also because of iatrogenic factors such as the use of PPIs and specialized tube feedings. If using intravenous $\mathrm{LT}_{4}$, the equivalent intravenous dose is approximately $75 \%$, assuming the enteral $\mathrm{LT}_{4}$ dose had achieved euthyroidism.

It should be noted that increased $\mathrm{LT}_{4}$ replacement therapy may lead to TSH normalization without normalization of $\mathrm{T}_{3}$ (594), presumably due to accelerated metabolism of $\mathrm{T}_{3}$. However, as the NTIS is considered adaptive, logic dictates that "correction" of the low $\mathrm{T}_{3}$ may not be an appropriate goal of replacement, even for patients with a known history of hypothyroidism being maintained on $\mathrm{LT}_{4}$. Indeed, using $\mathrm{LT}_{3}$ for replacement is problematic because the appropriate target range for serum $\mathrm{T}_{3}$ is not defined in this setting; furthermore, high doses of $\mathrm{T}_{3}$ have been linked to adverse outcomes (595). High-quality randomized placebo controlled trials of various TH regimens in NTIS addressing whether thyroid axis derangements are adaptive or pathophysiologic are needed but would be challenging to design. As patients recover from their critical nonthyroidal illness, a rebound in TSH can occur. Thus, proper dose adjustment requires serial TSH measurements and correlation with overall clinical status.

The possibility of adrenal insufficiency must also be considered in this setting, since treatment of hypothyroidism might accelerate cortisol metabolism (596). There are case reports of adrenal insufficiency unmasked by TH $(597,598)$.

\section{Myxedema Coma}

\section{1a. In patients with myxedema coma being treated with levothyroxine, what route of administration should be used?}

\section{- RECOMMENDATION}

Initial thyroid hormone replacement for myxedema coma should be levothyroxine given intravenously. A loading dose of 200-400 $\mu \mathrm{g}$ of levothyroxine may be given, with lower doses given for smaller or older patients and those with a history of coronary disease or arrhythmia. A daily replacement dose of $1.6 \mu \mathrm{g} / \mathrm{kg}$ body weight, reduced to $75 \%$ as long as it is being intravenously administered, can be given thereafter. Oral therapy or other enteral therapy if the oral route cannot be employed may be instituted after the patient improves clinically.

Strong recommendation. Low-quality evidence.

\section{1b. In patients with myxedema coma being treated with levothyroxine, should empiric glucocorticoid coverage be provided?}

\section{- RECOMMENDATION}

Empiric glucocorticoid coverage should be employed as part of the initial therapy for myxedema coma, with intravenous glucocorticoid administration, at doses appropriate for the stressed state, preceding levothyroxine administration.

Strong recommendation. Low-quality evidence.

\section{1c. In patients with myxedema coma being treated with levothyroxine, should liothyronine therapy also be initiated?}

\section{- RECOMMENDATION}

Given the possibility that thyroxine conversion to triiodothyronine may be decreased in patients with myxedema coma, intravenous liothyronine may be given in addition to levothyroxine. High doses should be avoided given the association of high serum triiodothyronine during treatment with mortality. A loading dose of 5-20 $\mu \mathrm{g}$ can be given, followed by a maintenance dose of $2.5-10 \mu \mathrm{g}$ every 8 hours, with lower doses chosen for smaller or older patients and those with a history of coronary artery disease or arrhythmia. Therapy can continue until the patient is clearly recovering (e.g., until the patient regains consciousness and clinical parameters have improved).

Weak recommendation. Low-quality evidence.

\section{1d. In patients with myxedema coma being treated with levothyroxine, what therapeutic endpoints should be targeted?}

\section{- RECOMMENDATION}

Intravenous levothyroxine treatment in severely hypothyroid patients may lead to improvement in cardiovascular, renal, pulmonary, and metabolic parameters within a week. Serum thyroxine and triiodothyronine concentrations may improve or normalize with a similar time frame, with more gradual improvement in serum TSH. Thus, the therapeutic endpoints in myxedema coma should be improved mental status, improved cardiac function, and improved pulmonary function. Measurement of thyroid hormones every 1-2 days is reasonable to ensure a favorable trajectory in the biochemical parameters. While optimal levels for serum TSH and thyroid hormones are not well defined in this circumstance, failure of TSH to trend down or for thyroid hormone levels to improve could be considered indications to increase levothyroxine therapy and/or add liothyronine therapy, whereas high serum triiodothyronine could be considered an indication to decrease therapy given safety concerns.

Weak recommendation. Low-quality evidence.

\section{Discussion of the clinical literature}

In the extreme case of myxedema coma, the literature clearly supports the use of TH replacement as the disease has a high mortality rate $(595,599)$. There are case reports demonstrating death without treatment (600-602). In the setting of myxedema coma TH therapy should be instituted, or increased if a patient is already receiving treatment. $\mathrm{LT}_{4}$ should be given initially. $\mathrm{LT}_{3}$ can be given as well, though case series suggest that higher doses of $\mathrm{LT}_{3}$ may be associated with adverse outcomes (595).

Therapy can and should be instituted based on clinical suspicion alone and should not be delayed while waiting for blood test results. If the patient has been taking $\mathrm{TH}$, it must be assumed that the dose or route is inappropriate, and more 
aggressive replacement instituted immediately. In this setting, primary hypothyroidism may itself be the inciting cause of critical illness or might complicate a nonthyroidal critical illness if unrecognized and untreated (603). Thyroid hormone replacement is vital for the survival of these patients, even though the NTIS physiology still applies; because hypothyroidism is considered a primary motivator of illness in this setting, concerns about abnormal $\mathrm{T}_{4}$ to $\mathrm{T}_{3}$ conversion become relevant, and replacement with $\mathrm{LT}_{3}$ may be more reasonable.

It must be remembered that the degree of TSH elevation may not be an accurate indicator of the severity of the hypothyroidism based on the wide range of serum TSH values seen in myxedema coma (595), possibly due to variable suppression of the hypothalamic-pituitary axis by the illness. Also, a lesser magnitude of TSH elevation may be seen in older patients (366), at least in the outpatient setting. An inappropriate TSH may also be seen if hypothyroidism is caused by TSH deficiency (e.g., secondary hypothyroidism in patients with panhypopituitarism).

Initial TH replacement for myxedema coma should be $\mathrm{LT}_{4}$ given intravenously. A loading dose of 200-400 $\mu \mathrm{g}$ of $\mathrm{LT}_{4}$ may be given, with lower doses given for smaller or older patients and those with a history of coronary disease or arrhythmia. A daily replacement dose of $1.6 \mu \mathrm{g} / \mathrm{kg}$ body weight, reduced to $75 \%$ as long as it is being intravenously administered, can be given thereafter.

A study of a group of hypothyroid patients with mean serum TSH values of approximately $80 \mathrm{mIU} / \mathrm{L}$ treated with $100 \mu \mathrm{g}$ intravenous $\mathrm{LT}_{4}$ daily showed normalization of $\mathrm{TH}$ levels in 4 days, with significant improvement in serum TSH and other clinical parameters within a week (604). These dramatic improvements occurred without a loading dose of $\mathrm{LT}_{4}$. With administration of a loading dose in patients with myxedema coma, there may be a greater increase in $\mathrm{T}_{4}$ and $\mathrm{T}_{3}$ based on the dose administered, the volume of distribution, and the occupancy of binding proteins. Therapeutic endpoints to target would mainly be those clinical parameters related to the various organ systems affected; for example, mental status, temperature, respiratory function, cardiovascular status, electrolytes, and biochemical measures of thyroid status. While equilibrium levels of TSH would not be anticipated until well after clinical improvement should be seen, serially measuring the serum $\mathrm{TSH}, \mathrm{FT}_{4}$ or $\mathrm{T}_{4}$, and $\mathrm{T}_{3}$ in patients being treated for myxedema coma could provide supportive evidence that the therapy is working and could ensure that very high levels of $\mathrm{T}_{3}$ are avoided. In addition, checking the serum $T_{4}$ and $T_{3}$ concentrations 1-2 days after initiation of therapy will allow assessment of the response to the loading dose. Therapy should be aggressive: if the clinical picture is not improving, $\mathrm{LT}_{4}$ could be increased and/or $\mathrm{LT}_{3}$ could be increased or added. Safety concerns include tachycardia, arrhythmia, and myocardial infarction. Oral therapy or other enteral therapy if the oral route cannot be employed may be instituted after the patient improves clinically.

Given the possibility that $\mathrm{T}_{4}$ conversion to $\mathrm{T}_{3}$ may be decreased in patients with myxedema coma, intravenous $\mathrm{LT}_{3}$ may be given in addition to $\mathrm{LT}_{4}$. High doses should be avoided given their association with mortality (595). A loading dose of 5-20 $\mu \mathrm{g}$ can be given, followed by a maintenance dose of $2.5-10 \mu \mathrm{g}$ every 8 hours, with lower doses chosen for smaller or older patients and those with a history of coronary artery disease or arrhythmia. If intravenous therapy with $\mathrm{LT}_{3}$ is initiated, there are no data addressing optimum duration of such therapy. However, it would seem reasonable to continue intravenous therapy with $\mathrm{LT}_{3}$ until there is a clinical response that indicates that the patient is clearly recovering, such as regained consciousness, improved mental status, improved pulmonary function, improved cardiac function, and improvement of clinical parameters. Use of intravenous $\mathrm{LT}_{3}$ monotherapy administered at doses of either 25 or $50 \mu \mathrm{g}$ daily in hypothyroid patients with serum TSH values in the range of $68-81 \mathrm{mIU} / \mathrm{L}$ lead to normalization of various cardiovascular, pulmonary, and metabolic parameters within a week (605). TSH values decreased significantly within the same time period, although less so than the TSH response to intravenous $\mathrm{LT}_{4}$ observed by the same authors in a similar cohort of patients (604).

Given the rarity of myxedema coma, randomized clinical trials are not feasible, and so recommendations regarding the type, route, and outcomes measures for replacement are based solely on expert opinion and case reports (595,599,606-619). In addition to aggressive TH replacement, it is widely accepted that supportive measures directed at treating hypothermia, hypoventilation, and hyponatremia are important. In addition to supportive measures for hypothermia, hypoventilation, volume depletion, hypoglycemia, and hyponatremia, most clinicians favor empiric treatment of concomitant conditions. Empiric glucocorticoid therapy at stress doses should be initiated at the start of therapy for myxedema coma. Serum cortisol should ideally be measured prior to initiation of therapy. Empiric broad-spectrum antibiotic coverage should be administered if sepsis is in the differential diagnosis.

\section{Low Triiodothyronine Concentrations in Hospitalized Patients}

\section{2a. In hospitalized adult patients exhibiting the "nonthyroidal illness syndrome," should thyroid hormone replacement be instituted with levothyroxine?}

\section{- RECOMMENDATION}

We recommend against the use of levothyroxine as a form of therapy for hospitalized patients experiencing critical illness exhibiting the nonthyroidal illness syndrome. The few randomized controlled trials comparing levothyroxine therapy to no treatment have not shown significant clinical benefit, and have raised safety concerns that limit support for this approach.

Strong recommendation. Moderate quality evidence.

\section{2b. In hospitalized adult patients exhibiting the "nonthyroidal illness syndrome," should thyroid hormone replacement be instituted with liothyronine?}

\section{- RECOMMENDATION}

We recommend against the use of liothyronine as a form of therapy for hospitalized patients experiencing critical illness exhibiting the nonthyroidal illness syndrome. Although low doses of liothyronine have not been linked to harm in clinical trials, data showing any significant clinical benefit are also lacking.

Weak recommendation. Moderate quality evidence. 


\section{Mechanistic background}

There seem to be differences between the acute and chronic phases of critical illness (28). During acute illness there are changes in TH binding, alteration in TH uptake by cells, decreased $T_{4}$ to $T_{3}$ conversion due to the effects of endogenous circulating cortisol, cytokines, free fatty acids, and various drugs on D1. There may also be increased $T_{3}$ catabolism due to changes in the deiodinases, namely D3, in peripheral tissues. All these effects lead to low circulating $T_{3}$ levels. As illness becomes more prolonged, superimposed upon these changes is central suppression of the hypothalamic pituitary axis, with decreases in circulating TSH and $T_{4}$

While decreased $T_{4}$ to $T_{3}$ conversion has been posited as an etiologic cause of the NTIS, more recent data suggest that increased $T_{3}$ catabolism because of ectopically up-regulated D3 in tissues such as liver, skeletal muscle, and heart has a greater impact in lowering $T_{3}(26)$. Studies in animal models have linked D3 induction to hypoxia, with direct up-regulation of the D3 gene by hypoxia-inducible factor 1, the major regulator of the hypoxic response $(363,620,621)$.

The underlying physiology of chronic NTIS involves a down-regulation of the hypothalamic-pituitary-thyroid axis such that production and release of THs decrease as the degree of illness worsens (622). Similar changes in circulating iodothyronines are seen regardless of the etiology of the underlying illness; most authorities have interpreted this teleologically to indicate that the depression of the axis represents a physiologic response, though dissenting voices exist. A minority view holds that the changes seen, while perhaps a common pathway, are nonetheless pathologic (623,624). The literature regarding mechanisms of the NTIS is based largely on rodent studies. Several important steps may ultimately lead to the NTIS. For example, caloric restriction leads to decreased leptin signaling, which in turn reduces TRH and TSH production $(625,626)$. Animal models of NTIS featuring either caloric restriction or endotoxin administration have been associated with increased D2 expression and activity in the mediobasal hypothalamus. An increase in D2-mediated $T_{4}$ to $T_{3}$ conversion would be expected to increase the local supply of $T_{3}$, thus decreasing TRH and TSH production rates (627). An increase in D2mediated $T_{4}$ to $T_{3}$ conversion would be expected to increase the supply of $T_{3}$ to important regulatory centers in the brain controlling the thyroid axis.

The extent to which central hypothyroidism, versus reduced D1 activity, and increased D3-mediated clearance in peripheral tissues cause NTIS remains to be determined, but one study of critically ill patients in which central hypothyroidism was corrected via TRH infusion suggests that the central effect predominates (622). Furthermore, a complete understanding of NTIS will not be possible without further studies of the effects of illness-related cytokine fluxes on TH binding, transport, and tissue activation, particularly in the settings of both acute inflammation and chronic severe illness $(28,628)$.

\section{Discussion of the clinical literature}

As patients with normal thyroid function become critically ill, a progressive decline in circulating $\mathrm{T}_{3}$ is observed, followed by decreases in serum $\mathrm{T}_{4}$ and $\mathrm{TSH}$ if the clinical course does not improve. This stereotypic pattern, known as NTIS or alternatively "euthyroid-sick syndrome," occurs without regard to the etiology of the primary illness, suggesting a physiologic, adaptive role. At the same time, since untreated hypothyroidism can itself lead to critical illness in the form of myxedema coma, debate continues as to whether NTIS patients might benefit from TH replacement.

Data from RCTs are limited, with studies involving relatively small numbers of patients suffering from heterogeneous life-threatening illnesses. These trials have failed to show benefits as far as major clinical endpoints such as survival are concerned, though it must be noted that their statistical power has typically been low. In some cases, replacement has been linked to harm, in particular with higher doses of $\mathrm{LT}_{3}$, and one study showed increased mortality with $\mathrm{LT}_{4}$ therapy in patients with acute renal failure (629). On the other hand, lower doses of $\mathrm{LT}_{3}$ have been suggested to alter certain clinical parameters in ways that could potentially be beneficial. In current practice, most clinicians interpret the data as not favoring $\mathrm{TH}$ replacement, and accept as dogma that NTIS is an adaptive process.

In order to evaluate whether hospitalized patients in the ICU with NTIS should be treated with $\mathrm{LT}_{4}$ or $\mathrm{LT}_{3}$, we first had to define which group of patients should be included. Given that our focus is adult patients in the ICU, we excluded studies of pediatric patients (less than 17 years old), brain dead potential organ donor patients (as this is a very specific clinical situation), and fasting patients. We excluded studies of perioperative (cardiac surgery) $\mathrm{LT}_{3}$ therapy from this section, since most of these studies included patients being given dopamine, which is known to suppress TSH and can cause iatrogenic hypothyroidism (630).

Randomized controlled data are limited with regards to this clinical question. Review of 10 recent review articles indicates that a large majority of experts do not advocate the initiation of $\mathrm{LT}_{3}$ therapy in this clinical setting $(28,356,447$, 624,631-636).

Kaptein et al. (447) have performed comprehensive reviews of this subject, published in 2009. They also performed a review of the postoperative literature in 2010 (632). They excluded studies that examined patients less than 18 years of age [except for Brent and Hershman (637)], studies of perioperative patients, studies with before and during study design, and studies of TH use for less than 24 hours. They also excluded studies for "data not extractable or end-points not included" as well (discussed later). Thus the results of seven RCTs $(629,637-642)$, the most recent of which was published in 2004 were included. Five of the studies utilized $\mathrm{LT}_{4}$ $(629,637,639-641)$, and two utilized LT $_{3}(638,642)$ (see Table 9). PubMed search of the literature revealed no relevant studies published since the Kaptein review.

Therefore, recommendation $22 \mathrm{~b}$ regarding the use of $\mathrm{LT}_{3}$ in critically ill subjects is based on only two published studies identified by Kaptein and colleagues (447). Becker et al. (638) studied burn patients (14 controls, 14 treated) in 1982, with the finding that $200 \mu \mathrm{g}$ oral $\mathrm{LT}_{3}$ did not alter mortality (four patients died in each group) or the high metabolic rate typically observed in burn patients. In 2004 Sirlak et al. (642) treated patients (40 controls, 40 treated) with $125 \mu \mathrm{g} \mathrm{LT}$ or placebo daily orally for 7 days prior to coronary artery bypass surgery and continued therapy until discharge. $T_{3}$ levels were higher in the treated group before surgery and after (but were 
Table 9. Characteristics of Randomized Controlled Trials Comparing "Clinical Endpoints" in Hospitalized Patients Treated with Either $\mathrm{LT}_{3} \mathrm{OR}_{\mathrm{LT}_{4}}$

\begin{tabular}{|c|c|c|c|c|}
\hline Reference & Treatment dosing & Patient population & Design & $\begin{array}{l}\text { No. of patients } \\
\text { randomized }\end{array}$ \\
\hline 638 & $\begin{array}{l}\mathrm{T}_{3}: 200 \mu \mathrm{g} / \mathrm{d} \\
\text { Dosing: once daily }\end{array}$ & Burn patients & Parallel, blinded & 28 \\
\hline 637 & $\begin{array}{l}\mathrm{T}_{4}: 1.5 \mu \mathrm{g} / \mathrm{kg} \\
\text { Dosing: once daily }\end{array}$ & Mixed ICU patients & Parallel, unblinded & 23 \\
\hline 640 & $\begin{array}{l}\mathrm{T}_{4}: 100 \mu \mathrm{g} / \mathrm{d} \\
\text { Dosing: once daily }\end{array}$ & Cardiomyopathy & Parallel, blinded & 20 \\
\hline 639 & $\begin{array}{l}\mathrm{T}_{4}: 100 \mu \mathrm{g} / \mathrm{d} \\
\text { Dosing: once daily }\end{array}$ & Cardiomyopathy & Parallel, blinded & 20 \\
\hline 644 & $\begin{array}{l}\mathrm{T}_{4}: 150 \mu \mathrm{g} / \mathrm{d} \\
\text { Dosing: twice daily }\end{array}$ & Acute renal failure & Parallel, blinded & 59 \\
\hline 641 & $\begin{array}{l}\mathrm{T}_{4}: 50 \mu \mathrm{g} / \mathrm{d} \\
\text { Dosing: once daily }\end{array}$ & CHF III/IV & Parallel, unblinded & 28 \\
\hline 642 & $\begin{array}{l}\mathrm{T}_{3}: 125 \mu \mathrm{g} / \mathrm{d} \\
\text { versus placebo }\end{array}$ & Cardiac surgery patients & Parallel, blinded & 80 \\
\hline
\end{tabular}

$\mathrm{CHF}$, congestive heart failure; ICU, intensive care unit.

similar during cardiopulmonary bypass). Inotropic requirements were decreased in the $\mathrm{T}_{3}$ group. Length of ICU stay was decreased in the $T_{3}$ group. Three patients died in each group. Atrial fibrillation was not increased in the $\mathrm{T}_{3}$ group. Morbidity and mortality were otherwise unaffected.

One notable study by Pingitore and colleagues (643) published in 2008 was excluded by the Kaptein et al. (447) analysis for "data not extractable." The endpoints were hemodynamic and neurohormonal testing. This trial (10 control, $10 \mathrm{~T}_{3}$ treated) employed intravenous infusion of $\mathrm{LT}_{3}$ for 3 days such that levels were higher than normal on day 1 , then normal. No adverse outcomes were reported with $\mathrm{T}_{3}$. It should be noted that studies with $\mathrm{LT}_{3}$ administration $<24$ hours were omitted from the analysis by Kaptein et al., as were patients treated with $\mathrm{LT}_{3}$ in the perioperative period (in subjects who may or may not have had NTIS). However, these studies are, nevertheless, cited by other authors to support the potential safety of short-term $\mathrm{LT}_{3}$ replacement. For example, Acker et al. (644) studied patients with delayed graft function in cadaveric renal transplantation, finding the intravenous infusion of $\mathrm{LT}_{3}$ (to levels still within the reference range) for 24 hours had no effect on the course, either beneficial or with regards to morbidity and mortality. This was excluded from the review by Kaptein et al. given the short duration of therapy.

De Groot's review (624) suggests that if $\mathrm{LT}_{3}$ therapy is to be initiated, it should be at a "replacement dose" of approximately $50 \mu \mathrm{g} / \mathrm{d}$ in divided doses. This is much lower than used in the two trials previously mentioned $(638,642)$. The review also suggests that $\mathrm{T}_{3}$ be followed and adjusted such that it is " at least low normal" before the next scheduled dose. De Groot (624) also advocates starting $\mathrm{LT}_{4}$, though no data for use of combination therapy in this situation could be identified. Interestingly, one postmortem study analyzing 31 patients that had died in the ICU and had been treated with $150 \mu \mathrm{g} \mathrm{T}_{4}$ and $0.6 \mu \mathrm{g} \mathrm{T}_{3} / \mathrm{kg}$ daily compared to 48 patients that had not been treated with THs, provided clear evidence for tissue specific regulation of tissue $\mathrm{T}_{4}$ and $\mathrm{T}_{3}$ concentrations in critical illness (645). Patients treated with $\mathrm{T}_{4}$ and $\mathrm{T}_{3}$ had approximately 1.5 times higher levels of serum and muscle
$T_{3}$, whereas liver $T_{3}$ concentrations were more than 4 times higher in patients receiving $\mathrm{T}_{4}$ and $\mathrm{T}_{3}$ therapy. However, this study did not address any potential benefits of TH use because all subjects died in the ICU, and there was no appropriate control group directed to answering this question.

\section{2c. In hospitalized adult patients with cardiac dysfunction, such as advanced heart failure, and low serum triiodothyronine concentrations, should thyroid hormone replacement be instituted with liothyronine?}

\section{- RECOMMENDATION}

We recommend against the routine use of liothyronine as a form of therapy for hospitalized patients with heart failure and low serum triiodothyronine concentrations given the mixed data from short-term trials, the hypothetical risks, and pending further randomized trials confirming benefit and safety.

\section{Weak recommendation. Moderate quality evidence.}

\section{Discussion of the clinical literature}

The clinical findings of both hypothyroidism and hyperthyroidism illustrate the dramatic effect of $\mathrm{T}_{3}$ alterations on cardiac output, contractility, vascular resistance, and blood pressure, as well as cholesterol metabolism (646). On a cellular level, similar to other tissues, $\mathrm{T}_{3}$ has both genomic and nongenomic effects on the cardiac myocyte. However, cardiac cells may be more reliant on adequate serum $\mathrm{T}_{3}$ levels for normal function based on data suggesting decreased transport of $\mathrm{T}_{4}$ into the cardiac myocyte (647). The role of $\mathrm{T}_{3}$ in cardiovascular disease is highlighted by NTIS in which the physiologic response to illnesses such as heart failure are characterized by a decrease in total and free $\mathrm{T}_{3}$ with normal $\mathrm{T}_{4}$ and TSH levels. NTIS is typically considered an adaptive response; however, there is continued discussion over whether $\mathrm{LT}_{3}$ replacement therapy may be worthwhile in an effort to reduce the negative consequences associated with NTIS including alterations in cardiac tissue remodeling (646). 
Hamilton et al. (648) reported on the safety of short-term $\mathrm{LT}_{3}$ infusion in 23 patients with advanced heart failure showing that administration of $\mathrm{LT}_{3}$ (cumulative dose $0.15-2.7 \mu \mathrm{g} / \mathrm{kg}$ ) was well tolerated with no significant change in heart rate, metabolic rate, or core temperature. In addition, the $\mathrm{LT}_{3}$ infusion was associated with increased cardiac output and reduced systemic vascular resistance in patients who received the largest dose (648). Subsequently, in the only randomized, placebo-controlled study to date, Pingitore et al. (643) reported a similar positive effect of $\mathrm{LT}_{3}$ on cardiac function in 20 patients with dilated cardiomyopathy. At a starting dose of $20 \mu \mathrm{g} / \mathrm{m}^{2}$, adjusted to obtain a normal serum $\mathrm{T}_{3}$ level, $\mathrm{LT}_{3}$ infusion for a 3-day period was associated with an increase in left-ventricular end-diastolic volume and stroke volume as well as reduced plasma noradrenaline, n-terminal pro-B-type natriuretic peptide, and aldosterone when compared to placebo controls. Again, there was no increase in heart rate or other side effects associated with $\mathrm{LT}_{3}$ therapy (643), but cardiovascular outcomes with longer-term therapy are needed. While these studies are encouraging (649), the question remains whether serum $\mathrm{T}_{3}$ may be better considered as a marker of disease status rather than a focus of therapy. The recent study by D'Aloia et al. (650) highlights this consideration where short-term dobutamine therapy resulted in statistically improved cardiac status associated with reduced brain natriuretic peptide reduction and increased $\mathrm{FT}_{3}$ levels.

\section{SECTION IV. USE OF THYROID HORMONE ANALOGS}

Thyroid Hormone Analogs and Euthyroid Patients

\section{Should thyroid hormone analog therapy be used in euthyroid individuals with non- hypothyroid-related medical conditions (such as dyslipidemia) based on current evidence?}

\section{- RECOMMENDATION}

Although preclinical data suggest that the concept of thyromimetic use for treatment of non-hypothyroidrelated medical conditions may be promising, we recommend against the use of such drugs outside of the research setting, due to concerns about the lack of clear benefit or excessive side effects of currently available preparations.

\section{Strong recommendation. Low-quality evidence.}

\section{Mechanistic background}

$T_{3}$ exerts its genomic effects via binding to its nuclear TRs (9). TRs function as hormone-modulated transcription factors, and their binding interactions with target genes either activate or repress transcription. There are two TR isoforms, TR $\alpha$ and $T R \beta$, encoded by separate genes. TR mRNAs can be alternatively spliced; in humans, three main splice variants are found, namely TR $\alpha 1, T R \alpha 2$, and TR $\beta 2$. Of note, these TR isoforms are differentially expressed throughout the body such that some tissues can be said to be dominant with respect to a given TR isoform $(8,9)$. For example, TR $\beta$ is the prevalent isoform in the liver and pituitary, whereas TR $\alpha$ is predominant in the heart, brain, and the skeleton. Thyroid hormone analogs may have significantly greater affinity for TR $\beta$ than TR $\alpha$, in the order of 22-fold greater for eprotirome (651), 10-fold greater for sobetirome (652), 14- to 15-fold greater for KB141 (653) and
MB07344 (654), and 40-fold greater for GC-24 (655). The various TH analogs, their relative selectivity for the TR $\beta$, and potential uses based on this selectivity are shown in Table 10.

Thyroid hormone analogs thus have potential clinical utility because they may promote tissue-specific effects (656). A tantalizing goal of research in this area has been to determine whether some of the beneficial effects of $T_{3}$ excess can be isolated pharmacologically, while avoiding unwanted effects. For example, given the predominance of TR $\beta$ in the liver, it has been postulated that selective TR $\beta$ agonist drugs could be used to lower cholesterol without the adverse TR $\alpha$-driven cardiac and skeletal effects that would be seen with pharmacologic $T_{3}$ administration. Animal studies using TR $\beta$ agonists have verified that this strategy is indeed possible (657-659). Furthermore, preclinical studies show that highly TRß-selective agonists may have other metabolic benefits, such as accelerating energy expenditure in rodents by increasing metabolic rate (660,661), reducing body weight (662), and conferring protection against diet-induced obesity (660).

Thyroid hormone analogs have also been studied because they may have higher binding affinities for the TRs than does $T_{3}$. For example, bindings studies suggest that TRIAC exhibits $\sim 3.5$-fold greater affinity for TR $\beta$ and $\sim 1.5$-fold greater affinity for TR $\alpha$ compared to $T_{3}$ (579), spurring interest in this compound in the treatment of RTH (663). On the other hand, 3,5-diiodothyropropionic acid (DITPA) is an analog with much lower binding affinity for TRs than $T_{3}$ (664). A number of animal studies have suggested that DITPA may have some degree of cardioselectivity, though the mechanism is unclear given that this drug binds TR $\alpha$ and TR $\beta$ with similar affinity (664). Studies of DITPA in animals have shown some promising effects such as improved left ventricular performance, reduced end-diastolic pressure, and cardiac remodeling after myocardial infarction $(665,666)$. However, one study of postischemic cardiac function showed

Table 10. The Selectivity and Potential Uses of ThyroId Hormone Analogs

\begin{tabular}{|c|c|c|}
\hline Analog & $\begin{array}{l}\text { Selectivity for } \\
\text { TR } \beta \text { (compared } \\
\text { with TR } \alpha)\end{array}$ & Potential use \\
\hline $\begin{array}{l}\text { Eprotirome } \\
\text { (KB2115) }\end{array}$ & 22-fold greater & Cholesterol lowering \\
\hline DIPTA & Equal affinity & $\begin{array}{l}\text { Cholesterol lowering } \\
\text { Cardiac inotropic }\end{array}$ \\
\hline $\begin{array}{l}\text { GC-1 } \\
\quad \text { (Sobetirome) }\end{array}$ & 10-fold greater & $\begin{array}{l}\text { Cholesterol lowering } \\
\text { Obesity treatment }\end{array}$ \\
\hline KB141 & 14-fold greater & Cholesterol lowering \\
\hline MB07344 & 15- fold greater & Cholesterol lowering \\
\hline GC-24 & 40-fold greater & Obesity treatment \\
\hline TRIAC & 2- to 3-fold greater & RTH \\
\hline Tetrac & $\begin{array}{l}\text { Metabolized } \\
\text { to TRIAC }\end{array}$ & RTH \\
\hline $\mathrm{CO} 23$ & $\begin{array}{l}\mathrm{TR} \beta \text { vs. TR } \alpha \\
\text { selectivity not } \\
\text { seen in rodents }\end{array}$ & $\begin{array}{l}\text { Effects on behavior } \\
\text { (if } \mathrm{TR} \alpha \text {-selective) }\end{array}$ \\
\hline
\end{tabular}

DIPTA, 3,5-diiodothyropropionic acid; RTH, resistance to thyroid hormone; TR $\alpha$, thyroid hormone receptor $\alpha$; TR $\beta$, thyroid hormone receptor $\beta$; TRIAC, 3,5,3'-triiodothyroacetic acid or tiratricol. 
an increased incidence of arrhythmias during ischemia and reperfusion with the use of DITPA (667).

\section{Discussion of the clinical literature}

A number of TH analogs have been evaluated in clinical trials. While several have shown potentially beneficial effects, significant side effects have also been seen that have limited enthusiasm with regards to their further development for clinical use. Clinical studies of eprotirome and other TR $\beta$-selective agonists or liver-specific TR ligands show consistently that there is a therapeutic window through which these drugs lower serum cholesterol without significantly affecting cardiovascular parameters or bone metabolism, and only minimally affecting TH economy $(668,669)$. Eprotirome therapy was associated with decreases in serum LDL, apolipoprotein B, triglycerides, and $\mathrm{Lp}$ (a) lipoprotein when added to statin therapy (668) and given as single therapy in primary hypercholesterolemia (670). However, a recent phase III trial of eprotirome for patients with heterozygous familial hypercholesterolemia (NCT01410383) was terminated in February 2012 after some liver injury was seen in participants and after dog toxicology studies revealed that 12 months of exposure to the drug led to cartilage damage (671). Although cartilage damage was not seen in study participants, these two findings lead the company to terminate all clinical trials with this drug (672).

Sobetirome (GC-1) is a highly selective TR $\beta$ agonist that currently has an active investigational new drug status. A clinical trial started at Oregon Health Sciences University in the fall of 2012 (NCT01787578) in patients with X-linked adrenoleukodystrophy based on preclinical data indicating that $\mathrm{T}_{3}$ or $\mathrm{TR} \beta$ agonists up-regulate the gene $A B C D 2$; up-regulation of $A B C D 2$ can compensate for the mutation in $A B C D 1$ (adrenoleukodystrophy gene) carried by these patients.

DITPA has been tested in two clinical trials focused on its potential cardiovascular benefits in heart failure patients. Despite the promising effects in animal studies, although DITPA improved some hemodynamic parameters, there was no overall benefit in patients with congestive heart failure (673). In fact, two clinical trials (Titan PharmaceuticalsNCT00103519; VA- NCT00032643) were terminated in 2006 and 2009, respectively. Another clinical trial of DITPA in a heart failure population reported improved body weight and LDL levels, but also a high dropout rate and negative effects on the skeleton (674). Of interest, DITPA has been effective in reversing some of the abnormalities seen in four children with MCT8 deficiency treated with this agent on compassionate grounds (501). The success of this therapy is presumably due to the fact that DITPA can enter brain tissues without the need for the MCT8 transporter.

Two studies of TRIAC have been reported, showing that this analog can lower serum $\mathrm{FT}_{4}$ without a compensatory increase in serum TSH, suggesting a suppressive effect on TSH secretion. At the same time, plasma LDL and total cholesterol levels decreased while serum osteocalcin and urinary excretion of calcium and pyridinium cross-links increased $(675,676)$. Should future analogs have specific TSHsuppressive potential, they might be useful agents for the management of thyroid cancer.

It should be noted that none of the analogs have been tested in large randomized clinical trials in euthyroid individuals, and none are FDA approved. Thus, more research is needed with new analogs to see if the potential for tissue-specific pharmacologic control of TH action can be achieved.

\section{Thyroid Hormone Analogs and Resistance to Thyroid Hormone}

\section{4a. What are the clinical and biochemical goals for treatment of patients who have genetic syndromes of resistance to thyroid hormone?}

\section{- RECOMMENDATION}

The therapeutic goals of the treatment of patients with genetic syndromes of resistance to thyroid hormone are to improve the symptoms caused by excessive TR $\alpha$ signaling, while minimizing the symptoms caused by deficient TR $\beta$ signaling.

Weak recommendation. Low-quality evidence.

\section{4b. What is the role of thyroid hormone analogs in treating patients who have genetic syndromes of resistance to thyroid hormone?}

\section{- RECOMMENDATION}

Although preliminary data from small case series suggest that the use of TRIAC in patients with genetic syndromes of resistance to thyroid hormone may be promising, more clinical research is needed before the use of thyroid hormone analogs can be recommended for this indication.

\section{Weak recommendation. Low-quality evidence.}

\section{Mechanistic background}

RTH (OMIM identifier \# 188570) (677) is a rare genetic disease characterized by increased serum levels of THs and inappropriately normal or increased TSH (678). RTH is caused by inactivating mutations in $T R \alpha(R T H \alpha)$ or $T R \beta$ (RTH $\beta)$. Patients with RTH $\alpha$ have normal levels of TSH in combination with a low $\mathrm{FT}_{4}$ and high $\mathrm{T}_{3}$ and suffer from local hypothyroidism in tissues that predominantly express TR $\alpha$ (376,377). Patients with RTH $\beta$ have normal or elevated levels of TSH despite elevated levels of $\mathrm{FT}_{4}$ and $\mathrm{FT}_{3}$. Symptoms in these latter patients are due to a combination of low TH action in predominantly TR $\beta$-expressing tissues and $T H$ overexposure in TR $\alpha$-expressing tissues $(378,379)$.

The majority of RTH forms are caused by autosomal dominant mutations in the TR $\beta$ gene (679), but in a significant percentage no mutation was identified (380), suggesting that mutations in other loci may cause RTH (680-683). Approximately 1000 patients in 350 families with mutations in TR $\beta$ have been described to date. These mutations may be associated with impaired ability to bind $T_{3}$, and/or may prevent the normal TR-mediated gene transactivation. The mutant TR $\beta /$ corepressor complexes remain bound to the $T_{3^{-}}$ responsive genes and transcriptional repression cannot be lifted by the presence of $T_{3}$. Thus, mutant TR $\beta$ molecules exhibit a "dominant negative" effect.

TR $\beta$ mediates the negative TSH feedback mechanism in the pituitary gland. The lack of adequate negative feedback results in an inappropriately normal or elevated TSH and a hyperactive goiter with variable degrees of increased serum levels of $T_{4}$ and $T_{3}$. In effect, $R T H$ patients exhibit unbalanced hormonal signaling due to the combination of defective TR $\beta$ 
and otherwise normal TR $\alpha$ receptors exposed to supraphysiologic levels of TH. The result is a mix of hyperthyroid symptoms and signs arising from organs enriched with the $T R \propto$ such as the heart, skeletal muscle, and brain, and hypothyroid signs related to tissues such as the liver where the $\beta$-isoform is predominant (380).

Hypothetically, a TH analog with a preference for TR $\beta$ might be useful in treatment of RTH patients, since it would normalize TSH and lower TH production, while normalizing TH signaling directly in TR $\beta$ dominant tissues. Treatment with TRIAC, which has a higher affinity for TR $\beta$ than for TR $\alpha$ has been used in a number of patients based on this rationale.

\section{Discussion of the clinical literature}

Literature on the treatment of RTH is limited to case reports given the rarity of the syndrome. Most patients with RTH can be managed symptomatically with selective $\beta$ blockers to minimize symptoms of hyperthyroidism arising from $\mathrm{TR} \alpha$ dominant tissues such as tachycardia and tremor; in TR $\beta$-dominant tissues, the excess TH is balanced for the most part by the relative insensitivity of the mutant TR $\beta$. Quite often, RTH patients are erroneously diagnosed as thyrotoxic and treated with radioablation or thyroid surgery (684). In such cases, use of $\mathrm{LT}_{4}$ therapy requires supraphysiological dosing if TR $\beta$-dominant tissues are to be made "euthyroid." However, this will potentially lead to hyperthyroidism in the TR $\alpha$-dominant tissues (685). Thus, $\mathrm{LT}_{4}$ can be combined with $\beta$-blockers to prevent TR $\alpha$-related cardiac symptoms (380). Treatment must be individualized, and no target TH or TSH value can be defined a priori as adequate replacement. Indeed, the replacement therapy should be adjusted to the patient's symptoms and signs. Aside from the use of the $\mathrm{LT}_{3}$ suppression test (Werner's test) as a diagnostic dynamic test to distinguish between RTH and TSH-producing adenomas, $\mathrm{LT}_{3}$ is generally not considered part of therapy, although it has been employed as a treatment modality for children with RTH also affected by attention deficient hyperactivity disorder (686).

Small case series indicate that the use of TRIAC in RTH is effective in suppressing the TSH with no evidence of cardiac toxicity $(573,687)$. One case involved a treatment duration of 3 years (663). Unfortunately, this drug is not currently available in the United States. Hence the treatment options are limited to $\mathrm{LT}_{4}$ coupled with cardiac-selective $\beta$-blockers. Although not currently tested in humans, TR $\beta$-mutantselective analogs may represent a more targeted therapeutic option for the treatment of RTH (688).

\section{FUTURE DIRECTIONS}

Despite the advances that have occurred in the field of therapy for hypothyroidism, there are still many unanswered questions. For example, with respect to targeting biochemical parameters and achieving amelioration of symptoms and patient satisfaction, it is not known whether replicating an individual's set point as opposed to targeting a particular part of the laboratory reference would be beneficial. Even if this approach did optimize therapy, there is no current routine means of determining an individual's set point in advance of them developing thyroid dysfunction, precluding replication of these parameters during subsequent therapy. Similarly, it is possible that replication of the endogenous circadian rhythm in $\mathrm{T}_{3}$ may also optimize therapy. However, current exogenous therapies do not reproduce endogenous serum $\mathrm{T}_{4}$ excursions or serum $\mathrm{T}_{3}$ rhythms.

It has been clearly established that $\mathrm{LT}_{4}$ therapy results in a higher serum $\mathrm{T}_{4} / \mathrm{T}_{3}$ ratio than is observed in the nonathyreotic state. It is not known whether there is a detrimental effect of this abnormal serum $\mathrm{T}_{4} / \mathrm{T}_{3}$ ratio. For example, because $\mathrm{T}_{4}$ is a primary inhibitor of $\mathrm{D} 2$ activity, does excessive circulating $\mathrm{T}_{4}$ ultimately lower the activity of $\mathrm{D} 2$ and result in any adverse consequences?

Another question relates to the best marker of thyroid status. Serum TSH is considered to be the best marker currently available. However, there are specific instances in which a normal serum TSH may be documented despite biochemical parameters indicating that specific tissues are in a hypothyroid or hyperthyroid state. In addition, there is some evidence that serum $\mathrm{T}_{3}$ values in the lower part of the normal range may not always be reflected by elevated serum TSH values or even serum TSH values in the upper part of the normal range (39-43). A related issue, particularly in specific subpopulations, is that the biologic activity of serum TSH is not always concordant with its measured concentrations. Of particular note, the questions $7 \mathrm{a}-7 \mathrm{c}$ raised by task force members, which relate to the significance of serum $\mathrm{T}_{3}$ concentrations and the thyroid status of different tissues, could only be addressed with summary statements, not formal recommendations, illustrating the need for additional studies in these areas. Another consideration is that serum $\mathrm{T}_{3}$ levels are known to be affected by age and disease state, adding further difficulty to their interpretation.

Yet another aspect of treatment of hypothyroidism is how to assess tissue euthyroidism. Animal studies show that a normal serum $\mathrm{T}_{3}$ is not necessarily accompanied by a normal $T_{3}$ concentration in all tissues. The ability of tissue $T_{3}$ to mirror serum $T_{3}$ is dependent on the particular tissue's complement of deiodinases. Gene profiling can demonstrate the genomic actions of TH within particular tissues. However, commercially available tests to indicate tissue status in humans are not available.

If the premise that $\mathrm{LT}_{4}$ does not provide optimum therapy for hypothyroidism were true, it would be anticipated that some of the many trials of combination therapy would have yielded positive results. However, other than an individual patient preference for combination therapy seen in some trials, there have generally been few benefits documented. Moreover, even a study that appeared to show a benefit at 3 months no longer showed maintenance of this benefit at 12 months. This is an important consideration given that $\mathrm{TH}$ therapy is generally lifelong. It is interesting that, assuming trials of combination therapy have been driven by a concern about low serum $T_{3}$ levels, the serum $T_{3}$ response has not been rigorously assessed in many trials, and restoration of serum $\mathrm{T}_{3}$ levels to "normal" has generally not been an endpoint.

It is possible that trials have not been more successful because the correct population has not yet been targeted. This target might include those with genetic variants of the deiodinases or other TH signaling elements, or those with disproportionately lower serum $\mathrm{T}_{3}$ levels while taking $\mathrm{LT}_{4}$ monotherapy. If $N$-of- 1 trials were being considered these might also logically be conducted in those with low or low- 
normal serum $\mathrm{T}_{3}$ concentrations. It is also possible that trials of once or twice daily $\mathrm{LT}_{3}$ therapy added to $\mathrm{LT}_{4}$ monotherapy have not had more positive outcomes because of the nonphysiologic nature of the $\mathrm{LT}_{3}$ dosing. A sustained release preparation, or even a preparation with a new delivery system that mimics the normal diurnal rhythm of serum $\mathrm{T}_{4}$ and $\mathrm{T}_{3}$, may be necessary in order to reveal a benefit. Large, prospective, long-term studies examining clinically significant outcomes are needed to clarify the optimal formulation or combination of formulations of $\mathrm{TH}$ replacement therapies and which patients may benefit from any specific therapies. More research is also needed to identify biomarkers that are most strongly associated with clinically relevant endpoints, long-term outcomes, and benefits of therapies. In addition to studying clinically relevant endpoints in future studies, longterm parallel studies rather than shorter-term cross-over studies may be most helpful.

Finally, qualitative research may be informative in exploring the nature of patient preferences for particular $\mathrm{TH}$ preparations. There is an absence of qualitative research in the area of evaluating therapies for hypothyroidism, which raises questions about the validity of many of the quantitative instruments used in assessing patient well-being on various formulations and dosages. Qualitative data are traditionally used to form the basis for more precise quantitative instruments; they also help to inform about covert socio-ethical barriers to well-being, which may affect how data are collected and/or analyzed.

Clearly, there have been great advances in the understanding and management of $\mathrm{TH}$ replacement, but nevertheless more research is needed. Areas in which future research should be encouraged include, but are not limited to:

1) Strategies to avoid iatrogenic thyroid disease in individuals treated for hypothyroidism

2) Research into strategies to aid compliance with $\mathrm{LT}_{4}$ therapy

3) Better understanding of maternal-fetal physiology during pregnancy with development of improved titration of $\mathrm{LT}_{4}$ therapy in hypothyroid pregnant patients

4) Further studies of soft gel $\mathrm{LT}_{4}$ capsules to determine their proper place in the therapeutic armamentarium

5) Further study, and improved standardization, of compounded formulations of $\mathrm{LT}_{4}$ and/or $\mathrm{LT}_{3}$

6) Development of additional biomarkers of euthyroidism, which may supplement the use of serum TSH as a biomarker

7) Development of a better understanding of how $T_{3}$ levels are affected by age and disease status, with consideration of reference ranges indexed to age and health status

8) Clarification of the relative importance of maintaining specific serum $T_{3}$ concentrations

9) Research into the relationship between serum $T_{3}$ and $\mathrm{T}_{3}$ concentrations in specific tissues

10) Development of more accurate assays to measure serum concentrations of $\mathrm{FT}_{3}$, total $\mathrm{T}_{3}$, and $\mathrm{FT}_{4}$

11) Development of a sustained release $T_{3}$ preparation that can then be prospectively tested in clinical trials (e.g., in combination with $\mathrm{LT}_{4}$ in a physiologic ratio of about 14:1)
12) In the absence of the availability of a sustained release $\mathrm{T}_{3}$ preparation, study of when, if ever, the use of $\mathrm{LT}_{3}$ would be beneficial in selected patients with apparent decreased $\mathrm{T}_{4}$ to $\mathrm{T}_{3}$ conversion and disproportionately low serum $\mathrm{T}_{3}$ levels

13) Long-term outcome research using thyroid extracts that includes documentation of the consequences of excursions in serum $\mathrm{T}_{3}$ concentrations

14) Development of $\mathrm{TH}$ analogs with a favorable benefit to risk profile

15) Pursuit of research into developing thyroid stem cells as a potential avenue for understanding thyrocyte physiology and as a possible future treatment for hypothyroidism.

Answers to questions such as these will advance our understanding of how to effectively and completely reverse the derangements that are a hallmark of untreated hypothyroidism, and will contribute to our ability to improve the lives of our patients with hypothyroidism.

\section{ACKNOWLEDGMENTS}

J.J. is partially supported by federal funds (Grant no. UL1TR000101) from the National Center for Advancing Translational Science, NIH, through the Clinical and Translational Science Awards Program. A.S. holds a Health Services Research Chair, funded by Cancer Care Ontario. The task force members would like to express their gratitude to Sharleene Cano (ATA), who served as our staff liaison and supported our activities with professionalism and efficiency. We would also like to thank Bobbi Smith (ATA) and our ATA board liaison Dr. James Hennessey for their expert assistance and advice, respectively. We acknowledge the extraordinarily diligent review of these guidelines performed by reviewers from the journal Thyroid.

This final document was officially endorsed by: Ukrainian Association of Endocrine Surgeons; The Endocrine Society of Australia; Society of Surgical Oncology; Pediatric Endocrine Society; Latin American Thyroid Society; International Federation of Head and Neck Oncologic Societies; International Association of Endocrine Surgeons; British Nuclear Medicine Society; American Head and Neck Society; Italian Endocrine Society; and American Association of Endocrine Surgeons.

\section{AUTHOR DISCLOSURE STATEMENT}

J.J. and K.D.B. have conducted research on a triiodothyronine product (BCT303) funded by ITL Pharma (Kingsport, TN). They are also planning a research project using levothyroxine gel capsules funded by Institut Biochimique S.A., Lugano, Switzerland. They have no financial interests in either product. All other authors have no conflicts of interest.

\section{REFERENCES}

1. Stagnaro-Green A, Abalovich M, Alexander E, Azizi F, Mestman J, Negro R, Nixon A, Pearce EN, Soldin OP, Sullivan S, Wiersinga W 2011 Guidelines of the American Thyroid Association for the diagnosis and management of thyroid disease during pregnancy and postpartum. Thyroid 21:1081-1125. 
2. De Groot L, Abalovich M, Alexander EK, Amino N, Barbour L, Cobin RH, Eastman CJ, Lazarus JH, Luton D, Mandel SJ, Mestman J, Rovet J, Sullivan S 2012 Management of thyroid dysfunction during pregnancy and postpartum: an Endocrine Society clinical practice guideline. J Clin Endocrinol Metab 97:2543-2565.

3. Garber JR, Cobin RH, Gharib H, Hennessey JV, Klein I, Mechanick JI, Pessah-Pollack R, Singer PA, Woeber KA, American Association Of Clinical E, American Thyroid Association Taskforce On Hypothyroidism In A 2012 Clinical practice guidelines for hypothyroidism in adults: cosponsored by the American Association of Clinical Endocrinologists and the American Thyroid Association. Thyroid 22:1200-1235.

4. Biondi B, Cooper DS 2008 The clinical significance of subclinical thyroid dysfunction. Endocr Rev 29:76-131.

5. Wiersinga W, Duntas LH, Fadeyev VV, Nygaard B, Vanderpump MPJ 20122012 ETA Guidelines: the use of L-T4 + L-T3 in the treatment of hypothyroidism. Eur Thyroid J 1:55-71.

6. Jonklaas J, Burman KD, Bianco AC 2013 Treatment of Hypothyroidism: Possibilities on the Horizon (For The American Thyroid Association Spring Program Committee). Thyroid 23:ix-xi.

7. Qaseem A, Snow V, Owens DK, Shekelle P 2010 The development of clinical practice guidelines and guidance statements of the American College of Physicians: summary of methods. Ann Intern Med 153:194-199.

8. Yen PM 2001 Physiological and molecular basis of thyroid hormone action. Physiol Rev 81:1097-1142.

9. Brent GA 2012 Mechanisms of thyroid hormone action. J Clin Invest 122:3035-3043.

10. Braverman LE, Ingbar SH, Sterling K 1970 Conversion of thyroxine (T4) to triiodothyronine (T3) in athyreotic human subjects. J Clin Invest 49:855-864.

11. Bianco AC, Salvatore D, Gereben B, Berry MJ, Larsen PR 2002 Biochemistry, cellular and molecular biology, and physiological roles of the iodothyronine selenodeiodinases. Endocr Rev 23:38-89.

12. Mandel SJ, Berry MJ, Kieffer JD, Harney JW, Warne RL, Larsen PR 1992 Cloning and in vitro expression of the human selenoprotein, type I iodothyronine deiodinase. J Clin Endocrinol Metab 75:1133-1139.

13. Schneider MJ, Fiering SN, Thai B, Wu SY, St Germain E, Parlow AF, St Germain DL, Galton VA 2006 Targeted disruption of the type 1 selenodeiodinase gene (Dio1) results in marked changes in thyroid hormone economy in mice. Endocrinology 147:580-589.

14. Oppenheimer JH, Schwartz HL, Surks MI 1972 Propylthiouracil inhibits the conversion of L-thyroxine to L-triiodothyronine. An explanation of the antithyroxine effect of propylthiouracil and evidence supporting the concept that triiodothyronine is the active thyroid hormone. J Clin Invest 51:2493-2497.

15. Salvatore D, Bartha T, Harney JW, Larsen PR 1996 Molecular biological and biochemical characterization of the human type 2 selenodeiodinase. Endocrinology 137: 3308-3315.

16. Salvatore D, Tu H, Harney JW, Larsen PR 1996 Type 2 iodothyronine deiodinase is highly expressed in human thyroid. J Clin Invest 98:962-968.

17. Houstek J, Vizek K, Pavelka S, Kopecky J, Krejcova E, Hermanska J, Cermakova M 1993 Type II iodothyronine $5^{\prime}$-deiodinase and uncoupling protein in brown adipose tissue of human newborns. J Clin Endocrinol Metab 77:382-387.

18. Geffner DL, Azukizawa M, Hershman JM 1975 Propylthiouracil blocks extrathyroidal conversion of thyroxine to triiodothyronine and augments thyrotropin secretion in man. J Clin Invest 55:224-229.

19. Saberi M, Sterling FH, Utiger RD 1975 Reduction in extrathyroidal triiodothyronine production by propylthiouracil in man. J Clin Invest 55:218-223.

20. LoPresti JS, Eigen A, Kaptein E, Anderson KP, Spencer vCA, Nicoloff JT 1989 Alterations in 3,3'5'-triiodothyronine metabolism in response to propylthiouracil, dexamethasone, and thyroxine administration in man. J Clin Invest 84: 1650-1656.

21. Baqui MM, Gereben B, Harney JW, Larsen PR, Bianco AC 2000 Distinct subcellular localization of transiently expressed types 1 and 2 iodothyronine deiodinases as determined by immunofluorescence confocal microscopy. Endocrinology 141:4309-4312.

22. Curcio C, Baqui MM, Salvatore D, Rihn BH, Mohr S, Harney JW, Larsen PR, Bianco AC 2001 The human type 2 iodothyronine deiodinase is a selenoprotein highly expressed in a mesothelioma cell line. J Biol Chem 276: 30183-30187.

23. Silva JE, Dick TE, Larsen PR 1978 The contribution of local tissue thyroxine monodeiodination to the nuclear 3,5,3'-triiodothyronine in pituitary, liver, and kidney of euthyroid rats. Endocrinology 103:1196-1207.

24. Kaplan MM, Yaskoski KA 1980 Phenolic and tyrosyl ring deiodination of iodothyronines in rat brain homogenates. $\mathrm{J}$ Clin Invest 66:551-562.

25. Slominski A, Wortsman J, Kohn L, Ain KB, Venkataraman GM, Pisarchik A, Chung JH, Giuliani C, Thornton M, Slugocki G, Tobin DJ 2002 Expression of hypothalamicpituitary-thyroid axis related genes in the human skin. J Invest Dermatol 119:1449-1455.

26. Peeters RP, Wouters PJ, Kaptein E, van Toor H, Visser TJ, Van den Berghe G 2003 Reduced activation and increased inactivation of thyroid hormone in tissues of critically ill patients. J Clin Endocrinol Metab 88:3202-3211.

27. Huang SA, Bianco AC 2008 Reawakened interest in type III iodothyronine deiodinase in critical illness and injury. Nat Clin Pract Endocrinol Metab 4:148-155.

28. Mebis L, Van den Berghe G 2011 Thyroid axis function and dysfunction in critical illness. Best Pract Res Clin Endocrinol Metab 25:745-757.

29. Van den Berghe G 2014 Non-thyroidal illness in the ICU: a syndrome with different faces. Thyroid 24:1456-1465.

30. van der Deure WM, Peeters RP, Visser TJ 2010 Molecular aspects of thyroid hormone transporters, including MCT8, MCT10, and OATPs, and the effects of genetic variation in these transporters. J Mol Endocrinol 44:1-11.

31. Kendall E 1915 The isolation in crystalline form of the compound containing iodin, which occurs in the thyroid. JAMA 64:2042-2043.

32. Harington CR, Barger G 1927 Chemistry of thyroxine: constitution and synthesis of thyroxine. Biochem J 21:169-183.

33. Chalmers J, Dickson G, Elks J, Hems B 1949 The synthesis of thyroxine and related substances. Part V. A synthesis of L-thyroxine from L-tyrosine. J Chem Soc 3424-3433.

34. Pilo A, Iervasi G, Vitek F, Ferdeghini M, Cazzuola F, Bianchi R 1990 Thyroidal and peripheral production of 3,5,3'-triiodothyronine in humans by multicompartmental analysis. Am J Physiol 258:E715-726. 
35. Hays MT, Nielsen KR 1994 Human thyroxine absorption: age effects and methodological analyses. Thyroid 4:55-64.

36. Saberi M, Utiger RD 1974 Serum thyroid hormone and thyrotropin concentrations during thyroxine and triiodothyronine therapy. J Clin Endocrinol Metab 39:923-927.

37. Ain KB, Pucino F, Shiver TM, Banks SM 1993 Thyroid hormone levels affected by time of blood sampling in thyroxine-treated patients. Thyroid 3:81-85.

38. Fish LH, Schwartz HL, Cavanaugh J, Steffes MW, Bantle JP, Oppenheimer JH 1987 Replacement dose, metabolism, and bioavailability of levothyroxine in the treatment of hypothyroidism. Role of triiodothyronine in pituitary feedback in humans. N Engl J Med 316:764-770.

39. Jonklaas J, Davidson B, Bhagat S, Soldin SJ 2008 Triiodothyronine levels in athyreotic individuals during levothyroxine therapy. JAMA 299:769-777.

40. Woeber KA 2002 Levothyroxine therapy and serum free thyroxine and free triiodothyronine concentrations. J Endocrinol Invest 25:106-109.

41. Gullo D, Latina A, Frasca F, Le Moli R, Pellegriti G, Vigneri R 2011 Levothyroxine monotherapy cannot guarantee euthyroidism in all athyreotic patients. PLoS One 6:e22552.

42. Ito M, Miyauchi A, Morita S, Kudo T, Nishihara E, Kihara M, Takamura Y, Ito Y, Kobayashi K, Miya A, Kubota S, Amino N 2012 TSH-suppressive doses of levothyroxine are required to achieve preoperative native serum triiodothyronine levels in patients who have undergone total thyroidectomy. Eur J Endocrinol 167:373-378.

43. Alevizaki M, Mantzou E, Cimponeriu AT, Alevizaki CC, Koutras DA 2005 TSH may not be a good marker for adequate thyroid hormone replacement therapy. Wien Klin Wochenschr 117:636-640.

44. Leboeuf R, Perron P, Carpentier AC, Verreault J, Langlois MF 2007 L-T $_{3}$ preparation for whole-body scintigraphy: a randomized-controlled trial. Clin Endocrinol 67:839-844.

45. Dow KH, Ferrell BR, Anello C 1997 Quality-of-life changes in patients with thyroid cancer after withdrawal of thyroid hormone therapy. Thyroid 7:613-619.

46. Cotton GE, Gorman CA, Mayberry WE 1971 Suppression of thyrotropin (h-TSH) in serums of patients with myxedema of varying etiology treated with thyroid hormones. N Engl J Med 285:529-533.

47. Mandel SJ, Brent GA, Larsen PR 1993 Levothyroxine therapy in patients with thyroid disease. Ann Intern Med 119:492-502.

48. Singer PA, Cooper DS, Levy EG, Ladenson PW, Braverman LE, Daniels G, Greenspan FS, McDougall IR, Nikolai TF 1995 Treatment guidelines for patients with hyperthyroidism and hypothyroidism. Standards of Care Committee, American Thyroid Association. JAMA 273: 808-812.

49. Canaris GJ, Manowitz NR, Mayor G, Ridgway EC 2000 The Colorado thyroid disease prevalence study. Arch Intern Med 160:526-534.

50. Walsh JP, Ward LC, Burke V, Bhagat CI, Shiels L, Henley D, Gillett MJ, Gilbert R, Tanner M, Stuckey BG 2006 Small changes in thyroxine dosage do not produce measurable changes in hypothyroid symptoms, wellbeing, or quality of life: results of a double-blind, randomized clinical trial. J Clin Endocrinol Metab 91:2624-2630.

51. al-Adsani H, Hoffer LJ, Silva JE 1997 Resting energy expenditure is sensitive to small dose changes in patients on chronic thyroid hormone replacement. J Clin Endocrinol Metab 82:1118-1125.

52. Rodondi N, den Elzen WP, Bauer DC, Cappola AR, Razvi S, Walsh JP, Asvold BO, Iervasi G, Imaizumi M, Collet TH, Bremner A, Maisonneuve P, Sgarbi JA, Khaw KT, Vanderpump MP, Newman AB, Cornuz J, Franklyn JA, Westendorp RG, Vittinghoff E, Gussekloo J 2010 Subclinical hypothyroidism and the risk of coronary heart disease and mortality. JAMA 304:1365-1374.

53. Razvi S, Weaver JU, Butler TJ, Pearce SH 2012 Levothyroxine treatment of subclinical hypothyroidism, fatal and nonfatal cardiovascular events, and mortality. Arch Intern Med 172:811-817.

54. Hollowell JG, Staehling NW, Flanders WD, Hannon WH, Gunter EW, Spencer CA, Braverman LE 2002 Serum TSH, T(4), and thyroid antibodies in the United States population (1988 to 1994): National Health and Nutrition Examination Survey (NHANES III). J Clin Endocrinol Metab 87:489-499.

55. Wartofsky L, Dickey RA 2005 The evidence for a narrower thyrotropin reference range is compelling. J Clin Endocrinol Metab 90:5483-5488.

56. Surks MI, Goswami G, Daniels GH 2005 The thyrotropin reference range should remain unchanged. J Clin Endocrinol Metab 90:5489-5496.

57. Canaris GJ, Steiner JF, Ridgway EC 1997 Do traditional symptoms of hypothyroidism correlate with biochemical disease? J Gen Intern Med 12:544-550.

58. Zulewski H, Muller B, Exer P, Miserez AR, Staub JJ 1997 Estimation of tissue hypothyroidism by a new clinical score: evaluation of patients with various grades of hypothyroidism and controls. J Clin Endocrinol Metab 82:771-776.

59. Skibinsky J, Goldberg V, Sirito R, Demartini AJ, Parisier H, Sciegata MA 1972 The diagnosis utility of the QK d interval in thyroid disease. Horm Metab Res 4:371-373.

60. Rodbard D, Fujita T, Rodbard S 1967 Estimation of thyroid function by timing the arterial sounds. JAMA 201: 884-887.

61. Smallridge R 1996 Metabolic, physiologic, and clinical indexes of thyroid function. In: Braverman L (ed) Werner and Ingbar's The Thyroid: A Fundamental and Clinical Text. Lippincott-Raven, Philadelphia, pp 397-405.

62. Birkent H, Karacalioglu O, Merati AL, Akcam T, Gerek M 2008 Prospective study of the impact of thyroid hormone replacement on objective voice parameters. Ann Otol Rhinol Laryngol 117:523-527.

63. Lambert EH, Underdahl LO, Beckett S, Mederos LO 1951 A study of the ankle jerk in myxedema. J Clin Endocrinol Metab 11:1186-1205.

64. Boothby W 1929 Normal values of basal or standard metabolism. A modification of the Dubois standard. Am J Physiol 90:290-291.

65. Werner S 1955 Basal metabolism. In: The Thyroid: A Fundamental and Clinical Text. Hoeber-Harper, New York, NY, pp 125-136.

66. Billewicz WZ, Chapman RS, Crooks J, Day ME, Gossage J, Wayne E, Young JA 1969 Statistical methods applied to the diagnosis of hypothyroidism. Q J Med 38:255-266.

67. Saravanan P, Visser TJ, Dayan CM 2006 Psychological well-being correlates with free thyroxine but not free $3,5,3^{\prime}$ triiodothyronine levels in patients on thyroid hormone replacement. J Clin Endocrinol Metab 91:3389-3393.

68. Saravanan P, Chau WF, Roberts N, Vedhara K, Greenwood R, Dayan CM 2002 Psychological well-being in 
patients on 'adequate' doses of L-thyroxine: results of a large, controlled community-based questionnaire study. Clin Endocrinol 57:577-585.

69. Bell GM, Todd WT, Forfar JC, Martyn C, Wathen CG, Gow S, Riemersma R, Toft AD 1985 End-organ responses to thyroxine therapy in subclinical hypothyroidism. Clin Endocrinol 22:83-89.

70. Monzani F, Caraccio N, Del Guerra P, Casolaro A, Ferrannini E 1999 Neuromuscular symptoms and dysfunction in subclinical hypothyroid patients: beneficial effect of $\mathrm{LT}_{4}$ replacement therapy. Clin Endocrinol 51: 237-242.

71. Bianco AC, Anderson G, Forrest D, Galton VAD, Gereben B, Kim BW, Kopp PA, Liao XH, Obregon MJ, Peeters R, Refetoff S, Sharlin D, Simonides WS, Weiss RE, Williams G 2014 American Thyroid Association guide to investigating thyroid hormone economy and action in rodent and cell models. Thyroid 24:88-168.

72. Feng X, Jiang Y, Meltzer P, Yen PM 2000 Thyroid hormone regulation of hepatic genes in vivo detected by complementary DNA microarray. Mol Endocrinol 14:947-955.

73. Danzi S, Klein I 2002 Thyroid hormone-regulated cardiac gene expression and cardiovascular disease. Thyroid 12:467-472.

74. Clément K, Viguerie N, Diehn M, Alizadeh A, Barbe P, Thalamas C, Storey JD, Brown PO, Barsh GS, Langin D 2002 In vivo regulation of human skeletal muscle gene expression by thyroid hormone. Genome Res 12:281-291.

75. Beckett GJ, Kellett HA, Gow SM, Hussey AJ, Hayes JD, Toft AD 1985 Raised plasma glutathione S-transferase values in hyperthyroidism and in hypothyroid patients receiving thyroxine replacement: evidence for hepatic damage. Br Med J (Clin Res Ed) 291:427-431.

76. de Bruin TW, van Barlingen $H$, van Linde-Sibenius Trip M, van Vuurst de Vries AR, Akveld MJ, Erkelens DW 1993 Lipoprotein(a) and apolipoprotein B plasma concentrations in hypothyroid, euthyroid, and hyperthyroid subjects. J Clin Endocrinol Metab 76:121-126.

77. Klein I, Mantell P, Parker M, Levey GS 1980 Resolution of abnormal muscle enzyme studies in hypothyroidism. Am J Med Sci 279:159-162.

78. Langdahl BL, Loft AG, Eriksen EF, Mosekilde L, Charles P 1996 Bone mass, bone turnover and body composition in former hypothyroid patients receiving replacement therapy. Eur J Endocrinol 134:702-709.

79. Mason R, Hunt H, Hurxthal L 1930 Blood cholesterol values in hyperthyroidism and hypothyroidism-their significance. N Engl J Med 203:1273-1278.

80. Tajiri J, Shimada T, Naomi S, Umeda T, Sato T 1984 Hepatic dysfunction in primary hypothyroidism. Endocrinol Jpn 31:83-91.

81. Danese MD, Ladenson PW, Meinert CL, Powe NR 2000 Clinical review 115: effect of thyroxine therapy on serum lipoproteins in patients with mild thyroid failure: a quantitative review of the literature. J Clin Endocrinol Metab 85:2993-3001.

82. Serter R, Demirbas B, Korukluoglu B, Culha C, Cakal E, Aral Y 2004 The effect of L-thyroxine replacement therapy on lipid based cardiovascular risk in subclinical hypothyroidism. J Endocrinol Invest 27:897-903.

83. Monzani F, Caraccio N, Kozakowa M, Dardano A, Vittone F, Virdis A, Taddei S, Palombo C, Ferrannini E 2004 Effect of levothyroxine replacement on lipid profile and intima-media thickness in subclinical hypothyroidism: a double-blind, placebo- controlled study. J Clin Endocrinol Metab 89:2099-2106.

84. Razvi S, Ingoe L, Keeka G, Oates C, McMillan C, Weaver JU 2007 The beneficial effect of L-thyroxine on cardiovascular risk factors, endothelial function, and quality of life in subclinical hypothyroidism: randomized, crossover trial. J Clin Endocrinol Metab 92:1715-1723.

85. Slawik M, Klawitter B, Meiser E, Schories M, Zwermann O, Borm K, Peper M, Lubrich B, Hug MJ, Nauck M, Olschewski M, Beuschlein F, Reincke M 2007 Thyroid hormone replacement for central hypothyroidism: a randomized controlled trial comparing two doses of thyroxine (T4) with a combination of T4 and triiodothyronine. J Clin Endocrinol Metab 92:4115-4122.

86. Teixeira PF, Reuters VS, Ferreira MM, Almeida CP, Reis FA, Melo BA, Buescu A, Costa AJ, Vaisman M 2008 Treatment of subclinical hypothyroidism reduces atherogenic lipid levels in a placebo-controlled double-blind clinical trial. Horm Metab Res 40:50-55.

87. Cooper DS, Halpern R, Wood LC, Levin AA, Ridgway EC 1984 L-Thyroxine therapy in subclinical hypothyroidism. A double-blind, placebo-controlled trial. Ann Intern Med 101:18-24.

88. Biondi B, Fazio S, Palmieri EA, Carella C, Panza N, Cittadini A, Bone F, Lombardi G, Sacca L 1999 Left ventricular diastolic dysfunction in patients with subclinical hypothyroidism. J Clin Endocrinol Metab 84:2064-2067.

89. Monzani F, Di Bello V, Caraccio N, Bertini A, Giorgi D, Giusti C, Ferrannini E 2001 Effect of levothyroxine on cardiac function and structure in subclinical hypothyroidism: a double blind, placebo-controlled study. J Clin Endocrinol Metab 86:1110-1115.

90. Virtanen VK, Saha HH, Groundstroem KW, Salmi J, Pasternack AI 2001 Thyroid hormone substitution therapy rapidly enhances left-ventricular diastolic function in hypothyroid patients. Cardiology 96:59-64.

91. Brenta G, Mutti LA, Schnitman M, Fretes O, Perrone A, Matute ML 2003 Assessment of left ventricular diastolic function by radionuclide ventriculography at rest and exercise in subclinical hypothyroidism, and its response to L-thyroxine therapy. Am J Cardiol 91:1327-1330.

92. Yazici M, Gorgulu S, Sertbas Y, Erbilen E, Albayrak S, Yildiz O, Uyan C 2004 Effects of thyroxin therapy on cardiac function in patients with subclinical hypothyroidism: index of myocardial performance in the evaluation of left ventricular function. Int J Cardiol 95:135-143.

93. Adrees M, Gibney J, El-Saeity N, Boran G 2009 Effects of 18 months of $\mathrm{LT}_{4}$ replacement in women with subclinical hypothyroidism. Clin Endocrinol 71:298-303.

94. Hennessey JV 2003 Levothyroxine a new drug? Since when? How could that be? Thyroid 13:279-282.

95. Hennessey JV, Evaul JE, Tseng YC, Burman KD, Wartofsky L 1986 L-thyroxine dosage: a reevaluation of therapy with contemporary preparations. Ann Intern Med 105:11-15.

96. Food, Drug Administration. Advisory Committee for Pharmaceutical Science, Record of Proceedings. 2003. http:// www.fda.gov/ohrms/dockets/ac/03/transcripts/3926T2.pdf.

97. American Association of Clinical Endocrinologists, the Endocrine Society, and the American Thyroid Association Joint Position Statement on the Use and Interchangeability of Thyroxine Products, 2004. www.thyroid.org/ thyroxine-products-joint-position-statement/ (accessed May 20, 2012). 
98. Blakesley V, Awni W, Locke C, Ludden T, Granneman GR, Braverman LE 2004 Are bioequivalence studies of levothyroxine sodium formulations in euthyroid volunteers reliable? Thyroid 14:191-200.

99. Escalante DA, Arem N, Arem R 1995 Assessment of interchangeability of two brands of levothyroxine preparations with a third-generation TSH assay. Am J Med 98:374-378.

100. Dong BJ, Hauck WW, Gambertoglio JG, Gee L, White JR, Bubp JL, Greenspan FS 1997 Bioequivalence of generic and brand-name levothyroxine products in the treatment of hypothyroidism. JAMA 277:1205-1213.

101. Mayor GH, Orlando T, Kurtz NM 1995 Limitations of levothyroxine bioequivalence evaluation: analysis of an attempted study. Am J Ther 2:417-432.

102. Eisenberg M, Distefano JJ 2009 TSH-based protocol, tablet instability, and absorption effects on $\mathrm{LT}_{4}$ bioequivalence. Thyroid 19:103-110.

103. U.S. Food and Drug Administration. FDA acts to ensure thyroid drugs don't lose potency before expiration date. Available at: http://www.fda.gov/Drugs/DrugSafety/Post marketDrugSafetyInformationforPatientsandProviders/ucm 161259.htm

104. U.S. Food and Drug Administration. Questions and answers on levothyroxine sodium products. Available at: http:// www.fda.gov/Drugs/DrugSafety/PostmarketDrugSafety InformationforPatientsandProviders/ucm 161266.htm

105. U.S. Department of Health and Human Services, Food and Drug Administration, Center for Drug Evaluation and Research. Guidance for Industry: Levothyroxine sodium products, enforcement of August 14, 2001, compliance date and submission of new applications. Available at: http://www.fda.gov/downloads/drugs/guidancecompliance regulatoryinformation/guidances/ucm079807

106. Lomenick JP, Wang L, Ampah SB, Saville BR, Greenwald FI 2013 Generic levothyroxine compared with synthroid in young children with congenital hypothyroidism. J Clin Endocrinol Metab 98:653-658.

107. Carswell JM, Gordon JH, Popovsky E, Hale A, Brown RS 2013 Generic and brand-name L-thyroxine are not bioequivalent for children with severe congenital hypothyroidism. J Clin Endocrinol Metab 98:610-617.

108. Hennessey JV 2013 Generic vs name brand L-thyroxine products: interchangeable or still not? J Clin Endocrinol Metab 98:511-514.

109. Benvenga S 2012 (Soft) capsules of wisdom: preventing myo-inositol malabsorption caused by coffee. Expert Opin Drug Deliv 9:1177-1179.

110. Pabla D, Akhlaghi F, Zia H 2009 A comparative pHdissolution profile study of selected commercial levothyroxine products using inductively coupled plasma mass spectrometry. Eur J Pharm Biopharm 72:105-110.

111. Colucci P, D'Angelo P, Mautone G, Scarsi C, Ducharme MP 2011 Pharmacokinetic equivalence of a levothyroxine sodium soft capsule manufactured using the new food and drug administration potency guidelines in healthy volunteers under fasting conditions. Ther Drug Monit 33:355-361.

112. Vita R, Saraceno G, Trimarchi F, Benvenga S 2013 A novel formulation of L-thyroxine (L-T4) reduces the problem of L-T4 malabsorption by coffee observed with traditional tablet formulations. Endocrine 43:154-160.

113. Brancato D, Scorsone A, Saura G, Ferrara L, Di Noto A, Aiello V, Fleres M, Provenzano V 2014 Comparison of TSH Levels with liquid formulation versus tablet formu- lations of levothyroxine in the treatment of adult hypothyroidism. Endocr Pract 20:657-662.

114. Vita R, Benvenga S 2014 Tablet levothyroxine (L-T4) malabsorption induced by proton pump inhibitor; a problem that was solved by switching to L-T4 in soft gel capsule. Endocr Pract 20:e38-e41.

115. Cappelli C, Pirola I, Gandossi E, Formenti A, Castellano M 2014 Oral liquid levothyroxine treatment at breakfast: a mistake? Eur J Endocrinol 170:95-99.

116. Pirola I, Formenti AM, Gandossi E, Mittempergher F, Casella C, Agosti B, Cappelli C 2013 Oral liquid L-thyroxine (L-T4) may be better absorbed compared to L-T4 tablets following bariatric surgery. Obes Surg 23:1493-1496.

117. Bernareggi A, Grata E, Pinorini MT, Conti A 2013 Oral liquid formulation of levothyroxine is stable in breakfast beverages and may improve thyroid patient compliance. Pharmaceutics 5:621-633.

118. Hays MT 1991 Localization of human thyroxine absorption. Thyroid 1:241-248.

119. Hays MT 1968 Absorption of oral thyroxine in man. J Clin Endocrinol Metab 28:749-756.

120. Wenzel KW, Kirschsieper HE 1977 Aspects of the absorption of oral L-thyroxine in normal man. Metabolism 26:1-8.

121. Benvenga S, Bartolone L, Squadrito S, Lo Giudice F, Trimarchi F 1995 Delayed intestinal absorption of levothyroxine. Thyroid 5:249-253.

122. Benvenga S, Bartolone L, Pappalardo MA, Russo A, Lapa D, Giorgianni G, Saraceno G, Trimarchi F 2008 Altered intestinal absorption of L-thyroxine caused by coffee. Thyroid 18:293-301.

123. Liel Y, Harman-Boehm I, Shany S 1996 Evidence for a clinically important adverse effect of fiber-enriched diet on the bioavailability of levothyroxine in adult hypothyroid patients. J Clin Endocrinol Metab 81:857-859.

124. Bell DS, Ovalle F 2001 Use of soy protein supplement and resultant need for increased dose of levothyroxine. Endocr Pract 7:193-194.

125. Lilja JJ, Neuvonen M, Neuvonen PJ 2004 Effects of regular consumption of grapefruit juice on the pharmacokinetics of simvastatin. Br J Clin Pharmacol 58:56-60.

126. Perez CL, Araki FS, Graf H, de Carvalho GA 2013 Serum thyrotropin levels following levothyroxine administration at breakfast. Thyroid 23:779-784.

127. Chiu AC, Sherman SI 1998 Effects of pharmacological fiber supplements on levothyroxine absorption. Thyroid 8:667-671.

128. Bach-Huynh TG, Nayak B, Loh J, Soldin S, Jonklaas J 2009 Timing of levothyroxine administration affects serum thyrotropin concentration. J Clin Endocrinol Metab 94:3905-3912.

129. Bolk N, Visser TJ, Nijman J, Jongste IJ, Tijssen JG, Berghout A 2010 Effects of evening vs morning levothyroxine intake: a randomized double-blind crossover trial. Arch Intern Med 170:1996-2003.

130. Elliott DP 2001 Effect of levothyroxine administration time on serum TSH in elderly patients. Ann Pharmacother 35:529-532.

131. Rajput R, Chatterjee S, Rajput M 2011 Can levothyroxine be taken as evening dose? Comparative evaluation of morning versus evening dose of levothyroxine in treatment of hypothyroidism. J Thyroid Res 2011:505239.

132. Singh N, Singh PN, Hershman JM 2000 Effect of calcium carbonate on the absorption of levothyroxine. JAMA 283:2822-2825. 
133. Singh N, Weisler SL, Hershman JM 2001 The acute effect of calcium carbonate on the intestinal absorption of levothyroxine. Thyroid 11:967-971.

134. Zamfirescu I, Carlson HE 2011 Absorption of levothyroxine when coadministered with various calcium formulations. Thyroid 21:483-486.

135. Sachmechi I, Reich DM, Aninyei M, Wibowo F, Gupta G, Kim PJ 2007 Effect of proton pump inhibitors on serum thyroid-stimulating hormone level in euthyroid patients treated with levothyroxine for hypothyroidism. Endocr Pract 13:345-349.

136. Northcutt RC, Stiel JN, Hollifield JW, Stant EG Jr 1969 The influence of cholestyramine on thyroxine absorption. JAMA 208:1857-1861.

137. Weitzman SP, Ginsburg KC, Carlson HE 2009 Colesevelam hydrochloride and lanthanum carbonate interfere with the absorption of levothyroxine. Thyroid 19:77-79.

138. John-Kalarickal J, Pearlman G, Carlson HE 2007 New medications which decrease levothyroxine absorption. Thyroid 17:763-765.

139. Diskin CJ, Stokes TJ, Dansby LM, Radcliff L, Carter TB 2007 Effect of phosphate binders upon TSH and Lthyroxine dose in patients on thyroid replacement. Int Urol Nephrol 39:599-602.

140. Campbell NR, Hasinoff BB, Stalts H, Rao B, Wong NC 1992 Ferrous sulfate reduces thyroxine efficacy in patients with hypothyroidism. Ann Intern Med 117:1010-1013.

141. Shakir KM, Chute JP, Aprill BS, Lazarus AA 1997 Ferrous sulfate-induced increase in requirement for thyroxine in a patient with primary hypothyroidism. South Med J 90:637-639.

142. Sperber AD, Liel Y 1992 Evidence for interference with the intestinal absorption of levothyroxine sodium by aluminum hydroxide. Arch Intern Med 152:183-184.

143. Liel Y, Sperber AD, Shany S 1994 Nonspecific intestinal adsorption of levothyroxine by aluminum hydroxide. Am J Med 97:363-365.

144. Sherman SI, Tielens ET, Ladenson PW 1994 Sucralfate causes malabsorption of L-thyroxine. Am J Med 96:531535.

145. Campbell JA, Schmidt BA, Bantle JP 1994 Sucralfate and the absorption of L-thyroxine. Ann Intern Med 121:152.

146. Dietrich JW, Gieselbrecht K, Holl RW, Boehm BO 2006 Absorption kinetics of levothyroxine is not altered by proton-pump inhibitor therapy. Horm Metab Res 38:57-59.

147. Ananthakrishnan S, Braverman LE, Levin RM, Magnani B, Pearce EN 2008 The effect of famotidine, esomeprazole, and ezetimibe on levothyroxine absorption. Thyroid 18: 493-498.

148. Siraj ES, Gupta MK, Reddy SS 2003 Raloxifene causing malabsorption of levothyroxine. Arch Intern Med 163: 1367-1370.

149. Cooper JG, Harboe K, Frost SK, Skadberg Ø 2005 Ciprofloxacin interacts with thyroid replacement therapy. BMJ 330:1002.

150. Madhava K, Hartley A 2005 Hypothyroidism in thyroid carcinoma follow-up: orlistat may inhibit the absorption of thyroxine. Clin Oncol (R Coll Radiol) 17:492.

151. McLean M, Kirkwood I, Epstein M, Jones B, Hall C 1993 Cation-exchange resin and inhibition of intestinal absorption of thyroxine. Lancet 341:1286.

152. Bugdaci MS, Zuhur SS, Sokmen M, Toksoy B, Bayraktar B, Altuntas Y 2011 The role of Helicobacter pylori in patients with hypothyroidism in whom could not be achieved normal thyrotropin levels despite treatment with high doses of thyroxine. Helicobacter 16:124-130.

153. Centanni M, Gargano L, Canettieri G, Viceconti N, Franchi A, Delle Fave G, Annibale B 2006 Thyroxine in goiter, Helicobacter pylori infection, and chronic gastritis. N Engl J Med 354:1787-1795.

154. Checchi S, Montanaro A, Pasqui L, Ciuoli C, De Palo V, Chiappetta MC, Pacini F 2008 L-thyroxine requirement in patients with autoimmune hypothyroidism and parietal cell antibodies. J Clin Endocrinol Metab 93:465-469.

155. Centanni M, Marignani M, Gargano L, Corleto VD, Casini A, Delle Fave G, Andreoli M, Annibale B 1999 Atrophic body gastritis in patients with autoimmune thyroid disease: an underdiagnosed association. Arch Intern Med 159: 1726-1730.

156. Virili C, Bassotti G, Santaguida MG, Iuorio R, Del Duca SC, Mercuri V, Picarelli A, Gargiulo P, Gargano L, Centanni M 2012 Atypical celiac disease as cause of increased need for thyroxine: a systematic study. J Clin Endocrinol Metab 97:E419-422.

157. Collins D, Wilcox R, Nathan M, Zubarik R 2012 Celiac disease and hypothyroidism. Am J Med 125:278-282.

158. Caputo M, Brizzolara R, Schiavo M, Salmaso C, Pesce G, Bagnasco M 2006 Occurrence of overt celiac disease in the elderly following total thyroidectomy. J Endocrinol Invest 29:831-833.

159. Silva CM, Souza MV 2005 Autoimmune hypothyroidism nonresponsive to high doses of levothyroxine and severe hypocalcemia [in Portuguese]. Arq Bras Endocrinol Metabol 49:599-603.

160. Munoz-Torres M, Varsavsky M, Alonso G 2006 Lactose intolerance revealed by severe resistance to treatment with levothyroxine. Thyroid 16:1171-1173.

161. Radaeli Rde F, Diehl LA 2011 Increased levothyroxine requirement in a woman with previously well-controlled hypothyroidism and intestinal giardiasis. Arq Bras Endocrinol Metabol 55:81-84.

162. Csako G, McGriff NJ, Rotman-Pikielny P, Sarlis NJ, Pucino F 2001 Exaggerated levothyroxine malabsorption due to calcium carbonate supplementation in gastrointestinal disorders. Ann Pharmacother 35:1578-1583.

163. Michalaki MA, Gkotsina MI, Mamali I, Markantes GK, Faltaka A, Kalfarentzos F, Vagenakis AG, Markou KB 2011 Impaired pharmacokinetics of levothyroxine in severely obese volunteers. Thyroid 21:477-481.

164. Azizi F, Belur R, Albano J 1979 Malabsorption of thyroid hormones after jejunoileal bypass for obesity. Ann Intern Med 90:941-942.

165. Bevan JS, Munro JF 1986 Thyroxine malabsorption following intestinal bypass surgery. Int J Obes 10:245-246.

166. Topliss DJ, Wright JA, Volpe R 1980 Increased requirement for thyroid hormone after a jejunoileal bypass operation. Can Med Assoc J 123:765-766.

167. Rubio IG, Galrao AL, Santo MA, Zanini AC, MedeirosNeto G 2012 Levothyroxine absorption in morbidly obese patients before and after Roux-En-Y gastric bypass (RYGB) surgery. Obes Surg 22:253-258.

168. Gkotsina M, Michalaki M, Mamali I, Markantes G, Sakellaropoulos GC, Kalfarentzos F, Vagenakis AG, Markou KB 2013 Improved levothyroxine pharmacokinetics after bariatric surgery. Thyroid 23:414-419.

169. Hennessey JV, Burman KD, Wartofsky L 1985 The equivalency of two L-thyroxine preparations. Ann Intern Med 102:770-773. 
170. Copeland PM 1995 Two cases of therapeutic failure associated with levothyroxine brand interchange. Ann Pharmacother 29:482-485.

171. Hennessey JV, Malabanan AO, Haugen BR, Levy EG 2010 Adverse event reporting in patients treated with levothyroxine: results of the pharmacovigilance task force survey of the American Thyroid Association, American Association of Clinical Endocrinologists, and the Endocrine Society. Endocr Pract 16:357-370.

172. Curran PG, DeGroot LJ 1991 The effect of hepatic enzymeinducing drugs on thyroid hormones and the thyroid gland. Endocr Rev 12:135-150.

173. Oppenheimer JH, Bernstein G, Surks MI 1968 Increased thyroxine turnover and thyroidal function after stimulation of hepatocellular binding of thyroxine by phenobarbital. J Clin Invest 47:1399-1406.

174. Cavlieri RR, Sung LC, Becker CE 1973 Effects of phenobarbital on thyroxine and triiodothyronine kinetics in Graves' disease. J Clin Endocrinol Metab 37:308-316.

175. Ohnhaus EE, Burgi H, Burger A, Studer H 1981 The effect of antipyrine, phenobarbitol and rifampicin on thyroid hormone metabolism in man. Eur J Clin Invest 11:381-387.

176. Rootwelt K, Ganes T, Johannessen SI 1978 Effect of carbamazepine, phenytoin and phenobarbitone on serum levels of thyroid hormones and thyrotropin in humans. Scand J Clin Lab Invest 38:731-736.

177. Larsen PR, Atkinson AJ Jr, Wellman HN, Goldsmith RE 1970 The effect of diphenylhydantoin on thyroxine metabolism in man. J Clin Invest 49:1266-1279.

178. Blackshear JL, Schultz AL, Napier JS, Stuart DD 1983 Thyroxine replacement requirements in hypothyroid patients receiving phenytoin. Ann Intern Med 99:341-342.

179. Aanderud S, Strandjord RE 1980 Hypothyroidism induced by anti-epileptic therapy. Acta Neurol Scand 61:330-332.

180. Kushnir M, Weinstein R, Landau B, Shavit G, Bechar M, Gadoth N 1985 Hypothyroidism and phenytoin intoxication. Ann Intern Med 102:341-342.

181. Aanderud S, Myking OL, Strandjord RE 1981 The influence of carbamazepine on thyroid hormones and thyroxine binding globulin in hypothyroid patients substituted with thyroxine. Clin Endocrinol 15:247-252.

182. Surks MI, DeFesi CR 1996 Normal serum free thyroid hormone concentrations in patients treated with phenytoin or carbamazepine. A paradox resolved. JAMA 275:1495-1498.

183. De Luca F, Arrigo T, Pandullo E, Siracusano MF, Benvenga $S$, Trimarchi $F 1986$ Changes in thyroid function tests induced by 2 month carbamazepine treatment in L-thyroxine-substituted hypothyroid children. Eur J Pediatr 145:77-79.

184. Isley WL 1987 Effect of rifampin therapy on thyroid function tests in a hypothyroid patient on replacement L-thyroxine. Ann Intern Med 107:517-518.

185. Takasu N, Takara M, Komiya I 2005 Rifampin-induced hypothyroidism in patients with Hashimoto's thyroiditis. N Engl J Med 352:518-519.

186. McCowen KC, Garber JR, Spark R 1997 Elevated serum thyrotropin in thyroxine-treated patients with hypothyroidism given sertraline. N Engl J Med 337:1010-1011.

187. de Carvalho GA, Bahls SC, Boeving A, Graf H 2009 Effects of selective serotonin reuptake inhibitors on thyroid function in depressed patients with primary hypothyroidism or normal thyroid function. Thyroid 19:691-697.

188. de Groot JW, Zonnenberg BA, Plukker JT, van Der Graaf WT, Links TP 2005 Imatinib induces hypothyroidism in patients receiving levothyroxine. Clin Pharmacol Ther 78:433-438.

189. Schlumberger MJ, Elisei R, Bastholt L, Wirth LJ, Martins RG, Locati LD, Jarzab B, Pacini F, Daumerie C, Droz JP, Eschenberg MJ, Sun YN, Juan T, Stepan DE, Sherman SI 2009 Phase II study of safety and efficacy of motesanib in patients with progressive or symptomatic, advanced or metastatic medullary thyroid cancer. J Clin Oncol 27: 3794-3801.

190. Abdulrahman RM, Verloop H, Hoftijzer H, Verburg E, Hovens GC, Corssmit EP, Reiners C, Gelderblom H, Pereira AM, Kapiteijn E, Romijn JA, Visser TJ, Smit JW 2010 Sorafenib-induced hypothyroidism is associated with increased type 3 deiodination. J Clin Endocrinol Metab 95:3758-3762.

191. Torino F, Barnabei A, Paragliola R, Baldelli R, Appetecchia M, Corsello SM 2013 Thyroid dysfunction as an unintended side effect of anticancer drugs. Thyroid 23:1345-1366.

192. Figge J, Dluhy RG 1990 Amiodarone-induced elevation of thyroid stimulating hormone in patients receiving levothyroxine for primary hypothyroidism. Ann Intern Med 113:553-555.

193. Rosene ML, Wittmann G, Arrojo e Drigo R, Singru PS, Lechan RM, Bianco AC 2010 Inhibition of the type 2 iodothyronine deiodinase underlies the elevated plasma TSH associated with amiodarone treatment. Endocrinology 151:5961-5970

194. Lotti G, Delitala G, Devilla L, Alagna S, Masala A 1977 Reduction of plasma triiodothyronine (T3) induced by propranolol. Clin Endocrinol 6:405-410.

195. Westgren U, Ahren B, Burger A, Ingemansson S, Melander A 1977 Effects of dexamethasone, desoxycorticosterone, and ACTH on serum concentrations of thyroxine, 3,5,3'triiodothyronine and 3,3',5'-triiodothyronine. Acta Med Scand 202:89-92.

196. Jorgensen JO, Pedersen SA, Laurberg P, Weeke J, Skakkebaek NE, Christiansen JS 1989 Effects of growth hormone therapy on thyroid function of growth hormone-deficient adults with and without concomitant thyroxine-substituted central hypothyroidism. J Clin Endocrinol Metab 69:11271132.

197. Grunfeld C, Sherman BM, Cavalieri RR 1988 The acute effects of human growth hormone administration on thyroid function in normal men. J Clin Endocrinol Metab 67:1111-1114.

198. Howard D, La Rosa FG, Huang S, Salvatore D, Mulcahey M, Sang-Lee J, Wachs M, Klopper JP 2011 Consumptive hypothyroidism resulting from hepatic vascular tumors in an athyreotic adult. J Clin Endocrinol Metab 96:19661970.

199. Ruppe MD, Huang SA, Jan de Beur SM 2005 Consumptive hypothyroidism caused by paraneoplastic production of type 3 iodothyronine deiodinase. Thyroid 15: 1369-1372.

200. Maynard MA, Marino-Enriquez A, Fletcher JA, Dorfman DM, Raut CP, Yassa L, Guo C, Wang Y, Dorfman C, Feldman HA, Frates MC, Song H, Jugo RH, Taguchi T, Hershman JM, Larsen PR, Huang SA 2014 Thyroid hormone inactivation in gastrointestinal stromal tumors. N Engl J Med 370:1327-1334.

201. Arafah BM 2001 Increased need for thyroxine in women with hypothyroidism during estrogen therapy. N Engl J Med 344:1743-1749. 
202. Mamby CC, Love RR, Lee KE 1995 Thyroid function test changes with adjuvant tamoxifen therapy in postmenopausal women with breast cancer. J Clin Oncol 13:854-857.

203. Duntas LH, Mantzou E, Koutras DA 2001 Lack of substantial effects of raloxifene on thyroxine-binding globulin in postmenopausal women: dependency on thyroid status. Thyroid 11:779-782.

204. McKerron CG, Scott RL, Asper SP, Levy RI 1969 Effects of clofibrate (Atromid S) on the thyroxine-binding capacity of thyroxine-binding globulin and free thyroxine. J Clin Endocrinol Metab 29:957-961.

205. Azizi F, Vagenakis AG, Portnay GI, Braverman LE, Ingbar SH 1974 Thyroxine transport and metabolism in methadone and heroin addicts. Ann Intern Med 80:194-199.

206. van Seters AP, Moolenaar AJ 1991 Mitotane increases the blood levels of hormone-binding proteins. Acta Endocrinol (Copenh) 124:526-533.

207. Beex L, Ross A, Smals A, Kloppenborg P 1977 5fluorouracil-induced increase of total serum thyroxine and triiodothyronine. Cancer Treat Rep 61:1291-1295.

208. Narula HS, Carlson HE 2004 Capecitabine-induced abnormalities in thyroid function tests. Am J Med 116:855856.

209. Muller AF, Verhoeff A, Mantel MJ, De Jong FH, Berghout A 2000 Decrease of free thyroxine levels after controlled ovarian hyperstimulation. J Clin Endocrinol Metab 85:545-548.

210. Stuckey BG, Yeap D, Turner SR 2010 Thyroxine replacement during super-ovulation for in vitro fertilization: a potential gap in management? Fertil Steril 93:2414 e2411-2413.

211. Arafah BM 1994 Decreased levothyroxine requirement in women with hypothyroidism during androgen therapy for breast cancer. Ann Intern Med 121:247-251.

212. Burmeister LA, Goumaz MO, Mariash CN, Oppenheimer JH 1992 Levothyroxine dose requirements for thyrotropin suppression in the treatment of differentiated thyroid cancer. J Clin Endocrinol Metab 75:344-350.

213. Rosenbaum RL, Barzel US 1982 Levothyroxine replacement dose for primary hypothyroidism decreases with age. Ann Intern Med 96:53-55.

214. Devdhar M, Drooger R, Pehlivanova M, Singh G, Jonklaas J 2011 Levothyroxine replacement doses are affected by gender and weight, but not age. Thyroid 21:821-827.

215. Jonklaas J 2010 Sex and age differences in levothyroxine dosage requirement. Endocr Pract 16:71-79.

216. Olubowale O, Chadwick DR 2006 Optimization of thyroxine replacement therapy after total or near-total thyroidectomy for benign thyroid disease. Br J Surg 93:57-60.

217. Davis FB, LaMantia RS, Spaulding SW, Wehmann RE, Davis PJ 1984 Estimation of a physiologic replacement dose of levothyroxine in elderly patients with hypothyroidism. Arch Intern Med 144:1752-1754.

218. Loh JA, Wartofsky L, Jonklaas J, Burman KD 2009 The magnitude of increased levothyroxine requirements in hypothyroid pregnant women depends upon the etiology of the hypothyroidism. Thyroid 19:269-275.

219. Cunningham JJ, Barzel US 1984 Lean body mass is a predictor of the daily requirement for thyroid hormone in older men and women. J Am Geriatr Soc 32:204-207.

220. Sawin CT, Herman T, Molitch ME, London MH, Kramer SM 1983 Aging and the thyroid. Decreased requirement for thyroid hormone in older hypothyroid patients. Am J Med 75:206-209.
221. Baehr KM, Lyden E, Treude K, Erickson J, Goldner W 2012 Levothyroxine dose following thyroidectomy is affected by more than just body weight. Laryngoscope 122:834-838.

222. Heemstra KA, Hoftijzer HC, van der Deure WM, Peeters RP, Fliers E, Appelhof BC, Wiersinga WM, Corssmit EP, Visser TJ, Smit JW 2009 Thr92Ala polymorphism in the type 2 deiodinase is not associated with T4 dose in athyroid patients or patients with Hashimoto thyroiditis. Clin Endocrinol 71:279-283.

223. Kabadi UM 1987 Variability of L-thyroxine replacement dose in elderly patients with primary hypothyroidism. J Fam Pract 24:473-477.

224. Torlontano M, Durante C, Torrente I, Crocetti U, Augello G, Ronga G, Montesano T, Travascio L, Verrienti A, Bruno R, Santini S, D'Arcangelo P, Dallapiccola B, Filetti S, Trischitta V 2008 Type 2 deiodinase polymorphism (threonine 92 alanine) predicts L-thyroxine dose to achieve target thyrotropin levels in thyroidectomized patients. J Clin Endocrinol Metab 93:910-913.

225. Santini F, Pinchera A, Marsili A, Ceccarini G, Castagna MG, Valeriano R, Giannetti M, Taddei D, Centoni R, Scartabelli G, Rago T, Mammoli C, Elisei R, Vitti P 2005 Lean body mass is a major determinant of levothyroxine dosage in the treatment of thyroid diseases. J Clin Endocrinol Metab 90:124-127.

226. Gordon MB, Gordon MS 1999 Variations in adequate levothyroxine replacement therapy in patients with different causes of hypothyroidism. Endocr Pract 5:233-238.

227. Roos A, Linn-Rasker SP, van Domburg RT, Tijssen JP, Berghout A 2005 The starting dose of levothyroxine in primary hypothyroidism treatment: a prospective, randomized, double-blind trial. Arch Intern Med 165:1714-1720.

228. Alexander EK, Marqusee E, Lawrence J, Jarolim P, Fischer GA, Larsen PR 2004 Timing and magnitude of increases in levothyroxine requirements during pregnancy in women with hypothyroidism. N Engl J Med 351:241-249.

229. Kabadi UM, Kabadi MM 2001 Serum thyrotropin in primary hypothyroidism: a reliable and accurate predictor of optimal daily levothyroxine dose. Endocr Pract 7:16-18.

230. Kundra P, Burman KD 2012 The effect of medications on thyroid function tests. Med Clin North Am 96:283-295.

231. Wartofsky L 2005 Levothyroxine therapy for hypothyroidism: should we abandon conservative dosage titration? Arch Intern Med 165:1683-1684.

232. Pecina J, Garrison GM, Bernard M 2014 Levothyroxine dosage is associated with stability of TSH values. Am J Med 127:240-245.

233. Somwaru LL, Arnold AM, Joshi N, Fried LP, Cappola AR 2009 High frequency of and factors associated with thyroid hormone over-replacement and under-replacement in men and women aged 65 and over. J Clin Endocrinol Metab 94:1342-1345

234. Sawin CT, Geller A, Wolf PA, Belanger AJ, Baker E, Bacharach P, Wilson PW, Benjamin EJ, D'Agostino RB 1994 Low serum thyrotropin concentrations as a risk factor for atrial fibrillation in older persons. N Engl J Med 331:1249-1252.

235. Bauer DC, Ettinger B, Nevitt MC, Stone KL 2001 Risk for fracture in women with low serum levels of thyroidstimulating hormone. Ann Intern Med 134:561-568.

236. Flynn RW, Bonellie SR, Jung RT, MacDonald TM, Morris AD, Leese GP 2010 Serum thyroid-stimulating hormone concentration and morbidity from cardiovascular 
disease and fractures in patients on long-term thyroxine therapy. J Clin Endocrinol Metab 95:186-193.

237. Cappola AR, Ladenson PW 2003 Hypothyroidism and atherosclerosis. J Clin Endocrinol Metab 88:2438-2444.

238. Rodondi N, Newman AB, Vittinghoff E, de Rekeneire N, Satterfield S, Harris TB, Bauer DC 2005 Subclinical hypothyroidism and the risk of heart failure, other cardiovascular events, and death. Arch Intern Med 165:2460-2466.

239. Ladenson PW, Sherman SI, Baughman KL, Ray PE, Feldman AM 1992 Reversible alterations in myocardial gene expression in a young man with dilated cardiomyopathy and hypothyroidism. Proc Natl Acad Sci U S A 89:5251-5255.

240. Shakir KM, Turton D, Aprill BS, Drake AJ 3rd, Eisold JF 2000 Anemia: a cause of intolerance to thyroxine sodium. Mayo Clin Proc 75:189-192.

241. Magner J, Gerber P 1994 Urticaria due to blue dye in synthroid tablets. Thyroid 4:341.

242. Choi YH, Choi WY, Kang HC, Koh YI, Bae EH, Kim SW 2012 Drug rash induced by levothyroxine tablets. Thyroid 22:1090.

243. Chandurkar V, Shik J, Randell E 2008 Exacerbation of underlying hypothyroidism caused by proteinuria and induction of urinary thyroxine loss: case report and subsequent investigation. Endocr Pract 14:97-103.

244. Keating FR Jr, Parkin TW, Selby JB, Dickinson LS 1961 Treatment of heart disease associated with myxedema. Prog Cardiovasc Dis 3:364-381.

245. Hay I, Duick DS, Vlietstra RE, Maloney JD, Pluth JR 1981 Thyroxine therapy in hypothyroid patients undergoing coronary revascularization: a retrospective analysis. Ann Intern Med 95:456-457.

246. Walker EF, Lewine RR, Neumann C 1996 Childhood behavioral characteristics and adult brain morphology in schizophrenia. Schizophr Res 22:93-101.

247. Walker EA, Katon WJ, Hansom J, Harrop-Griffiths J, Holm L, Jones ML, Hickok L, Jemelka RP 1992 Medical and psychiatric symptoms in women with childhood sexual abuse. Psychosom Med 54:658-664.

248. Trimble S 2004 Somatoform Disorders: A Medicolegal Guide. Cambridge University Press, Cambridge, UK.

249. Arnd-Caddigan M 2006 Somatoform disorders and a history of abuse: comorbidity, dynamics, and practice implications. Journal of Social Work 6:21-31.

250. Weetman AP 2006 Whose thyroid hormone replacement is it anyway? Clin Endocrinol 64:231-233.

251. Waller E, Scheidt CE 2006 Somatoform disorders as disorders of affect regulation: a development perspective. Int Rev Psychiatry 18:13-24.

252. Waldinger RJ, Schulz MS, Barsky AJ, Ahern DK 2006 Mapping the road from childhood trauma to adult somatization: the role of attachment. Psychosom Med 68:129-135.

253. He Q, Heo M, Heshka S, Wang J, Pierson RN Jr, Albu J, Wang Z, Heymsfield SB, Gallagher D 2003 Total body potassium differs by sex and race across the adult age span. Am J Clin Nutr 78:72-77.

254. Turner MR, Camacho X, Fischer HD, Austin PC, Anderson GM, Rochon PA, Lipscombe LL 2011 Levothyroxine dose and risk of fractures in older adults: nested case-control study. BMJ 342: 2238 .

255. Surks MI, Hollowell JG 2007 Age-specific distribution of serum thyrotropin and antithyroid antibodies in the US population: implications for the prevalence of subclinical hypothyroidism. J Clin Endocrinol Metab 92:4575-4582.
256. Boucai L, Surks MI 2009 Reference limits of serum TSH and free $\mathrm{T} 4$ are significantly influenced by race and age in an urban outpatient medical practice. Clin Endocrinol 70:788-793.

257. Waring AC, Arnold AM, Newman AB, Buzkova P, Hirsch C, Cappola AR 2012 Longitudinal changes in thyroid function in the oldest old and survival: the cardiovascular health study all-stars study. J Clin Endocrinol Metab 97:3944-3950.

258. Gussekloo J, van Exel E, de Craen AJ, Meinders AE, Frolich M, Westendorp RG 2004 Thyroid status, disability and cognitive function, and survival in old age. JAMA 292:2591-2599.

259. Simonsick EM, Newman AB, Ferrucci L, Satterfield S, Harris TB, Rodondi N, Bauer DC 2009 Subclinical hypothyroidism and functional mobility in older adults. Arch Intern Med 169:2011-2017.

260. Rhee CM, Curhan GC, Alexander EK, Bhan I, Brunelli SM 2013 Subclinical hypothyroidism and survival: the effects of heart failure and race. J Clin Endocrinol Metab 98:2326-2336.

261. Somwaru LL, Arnold AM, Cappola AR 2011 Predictors of thyroid hormone initiation in older adults: results from the cardiovascular health study. J Gerontol A Biol Sci Med Sci 66:809-814.

262. Medici M, de Rijke YB, Peeters RP, Visser W, de Muinck Keizer-Schrama SM, Jaddoe VV, Hofman A, Hooijkaas $\mathrm{H}$, Steegers EA, Tiemeier H, Bongers-Schokking JJ, Visser TJ 2012 Maternal early pregnancy and newborn thyroid hormone parameters: the Generation R study. J Clin Endocrinol Metab 97:646-652.

263. Benhadi N, Wiersinga WM, Reitsma JB, Vrijkotte TG, van der Wal MF, Bonsel GJ 2007 Ethnic differences in TSH but not in free T4 concentrations or TPO antibodies during pregnancy. Clin Endocrinol 66:765-770.

264. La'ulu SL, Roberts WL 2007 Second-trimester reference intervals for thyroid tests: the role of ethnicity. Clin Chem 53:1658-1664.

265. Korevaar TI, Medici M, de Rijke YB, Visser W, de Muinck Keizer-Schrama SM, Jaddoe VW, Hofman A, Ross HA, Visser WE, Hooijkaas H, Steegers EA, Tiemeier H, Bongers-Schokking JJ, Visser TJ, Peeters RP 2013 Ethnic differences in maternal thyroid parameters during pregnancy: the Generation R Study. J Clin Endocrinol Metab 98:3678-3686.

266. Li C, Shan Z, Mao J, Wang W, Xie X, Zhou W, Li C, Xu B, Bi L, Meng T, Du J, Zhang S, Gao Z, Zhang X, Yang L, Fan C, Teng W 2014 Assessment of thyroid function during first-trimester pregnancy: what is the rational upper limit of serum TSH during the first trimester in Chinese pregnant women? J Clin Endocrinol Metab 99:73-79.

267. van Deventer HE, Soldin SJ 2013 The expanding role of tandem mass spectrometry in optimizing diagnosis and treatment of thyroid disease. Adv Clin Chem 61:127-152.

268. Kahric-Janicic N, Soldin SJ, Soldin OP, West T, Gu J, Jonklaas J 2007 Tandem mass spectrometry improves the accuracy of free thyroxine measurements during pregnancy. Thyroid 17:303-311.

269. Abalovich M, Gutierrez S, Alcaraz G, Maccallini G, Garcia A, Levalle O 2002 Overt and subclinical hypothyroidism complicating pregnancy. Thyroid 12:63-68.

270. Hallengren B, Lantz M, Andreasson B, Grennert L 2009 Pregnant women on thyroxine substitution are often dysregulated in early pregnancy. Thyroid 19:391-394. 
271. LaFranchi SH, Haddow JE, Hollowell JG 2005 Is thyroid inadequacy during gestation a risk factor for adverse pregnancy and developmental outcomes? Thyroid 15:60-71.

272. Haddow JE, Palomaki GE, Allan WC, Williams JR, Knight GJ, Gagnon J, O'Heir CE, Mitchell ML, Hermos RJ, Waisbren SE, Faix JD, Klein RZ 1999 Maternal thyroid deficiency during pregnancy and subsequent neuropsychological development of the child. N Engl J Med 341:549-555.

273. Idris I, Srinivasan R, Simm A, Page RC 2005 Maternal hypothyroidism in early and late gestation: effects on neonatal and obstetric outcome. Clin Endocrinol 63:560 565.

274. Pop VJ, Kuijpens JL, van Baar AL, Verkerk G, van Son MM, de Vijlder JJ, Vulsma T, Wiersinga WM, Drexhage HA, Vader HL 1999 Low maternal free thyroxine concentrations during early pregnancy are associated with impaired psychomotor development in infancy. Clin Endocrinol 50:149-155.

275. Yassa L, Marqusee E, Fawcett R, Alexander EK 2010 Thyroid hormone early adjustment in pregnancy (the THERAPY) trial. J Clin Endocrinol Metab 95:3234-3241.

276. Mandel SJ, Larsen PR, Seely EW, Brent GA 1990 Increased need for thyroxine during pregnancy in women with primary hypothyroidism. N Engl J Med 323:91-96.

277. Verga U, Bergamaschi S, Cortelazzi D, Ronzoni S, Marconi AM, Beck-Peccoz P 2009 Adjustment of L-T4 substitutive therapy in pregnant women with subclinical, overt or postablative hypothyroidism. Clin Endocrinol 70:798-802.

278. Reid SM, Middleton P, Cossich MC, Crowther CA, Bain E 2013 Interventions for clinical and subclinical hypothyroidism pre-pregnancy and during pregnancy. Cochrane Database Syst Rev 5:CD007752.

279. Vissenberg R, van den Boogaard E, van Wely M, van der Post JA, Fliers E, Bisschop PH, Goddijn M 2012 Treatment of thyroid disorders before conception and in early pregnancy: a systematic review. Hum Reprod Update 18:360-373.

280. Downing S, Halpern L, Carswell J, Brown RS 2012 Severe maternal hypothyroidism corrected prior to the third trimester is associated with normal cognitive outcome in the offspring. Thyroid 22:625-630.

281. Lafranchi S 1992 Thyroiditis and acquired hypothyroidism. Pediatr Ann 21:29, 32-29.

282. Harris KB, Pass KA 2007 Increase in congenital hypothyroidism in New York State and in the United States. Mol Genet Metab 91:268-277.

283. LaFranchi SH, Austin J 2007 How should we be treating children with congenital hypothyroidism? J Pediatr Endocrinol Metab 20:559-578.

284. Germak JA, Foley TP Jr 1990 Longitudinal assessment of L-thyroxine therapy for congenital hypothyroidism. J Pediatr 117:211-219.

285. Mathai S, Cutfield WS, Gunn AJ, Webster D, Jefferies C, Robinson E, Hofman P 2008 A novel therapeutic paradigm to treat congenital hypothyroidism. Clin Endocrinol 69:142-147.

286. Selva KA, Harper A, Downs A, Blasco PA, Lafranchi SH 2005 Neurodevelopmental outcomes in congenital hypothyroidism: comparison of initial T4 dose and time to reach target T4 and TSH. J Pediatr 147:775-780.

287. American Academy of Pediatrics, Committee on Genetics 2001 American Academy of Pediatrics: health supervision for children with Down syndrome. Pediatrics 107:442-449.
288. Hrytsiuk I, Gilbert R, Logan S, Pindoria S, Brook CG 2002 Starting dose of levothyroxine for the treatment of congenital hypothyroidism: a systematic review. Arch Pediatr Adolesc Med 156:485-491.

289. Kempers MJ, van der Sluijs Veer L, Nijhuis-van der Sanden MW, Kooistra L, Wiedijk BM, Faber I, Last BF, de Vijlder JJ, Grootenhuis MA, Vulsma T 2006 Intellectual and motor development of young adults with congenital hypothyroidism diagnosed by neonatal screening. J Clin Endocrinol Metab 91:418-424.

290. Wasniewska M, De Luca F, Cassio A, Oggiaro N, Gianino P, Delvecchio M, Aiazzi R, Stoppioni V, Lombardo F, Messina MF, Valenzise M, Arrigo T 2003 In congenital hypothyroidism bone maturation at birth may be a predictive factor of psychomotor development during the first Year of life irrespective of other variables related to treatment. Eur J Endocrinol 149:1-6.

291. Akcay T, Turan S, Guran T, Unluguzel G, Haklar G, Bereket A 2010 T4 plus T3 treatment in children with hypothyroidism and inappropriately elevated thyroidstimulating hormone despite euthyroidism on T4 treatment. Horm Res Paediatr 73:108-114.

292. Balapatabendi M, Harris D, Shenoy SD 2011 Drug interaction of levothyroxine with infant colic drops. Arch Dis Child 96:888-889.

293. Jabbar MA, Larrea J, Shaw RA 1997 Abnormal thyroid function tests in infants with congenital hypothyroidism: the influence of soy-based formula. J Am Coll Nutr 16:280-282.

294. Balhara B, Misra M, Levitsky LL 2011 Clinical monitoring guidelines for congenital hypothyroidism: laboratory outcome data in the first year of life. J Pediatr 158: 532-537.

295. Leger J, Olivieri A, Donaldson M, Torresani T, Krude H, van Vliet G, Polak M, Butler G; ESPE-PES-SLEP-JSPE-APEGAPPES-ISPAE; Congenital Hypothyroidism Consensus Conference Group 2014 European Society for Paediatric Endocrinology consensus guidelines on screening, diagnosis, and management of congenital hypothyroidism. J Clin Endocrinol Metab 99:363-384.

296. de Vries L, Bulvik S, Phillip M 2009 Chronic autoimmune thyroiditis in children and adolescents: at presentation and during long-term follow-up. Arch Dis Child 94:33-37.

297. Nebesio TD, Wise MD, Perkins SM, Eugster EA 2011 Does clinical management impact height potential in children with severe acquired hypothyroidism? J Pediatr Endocrinol Metab 24:893-896.

298. Teng L, Bui H, Bachrach L, Lee P, Gagne N, Deal C, Wilson DM 2004 Catch-up growth in severe juvenile hypothyroidism: treatment with a GnRH analog. J Pediatr Endocrinol Metab 17:345-354.

299. Lomenick JP, El-Sayyid M, Smith WJ 2008 Effect of levothyroxine treatment on weight and body mass index in children with acquired hypothyroidism. J Pediatr 152:96-100.

300. Gharib H, Tuttle RM, Baskin HJ, Fish LH, Singer PA, McDermott MT American Association of Clinical Endocrinologists; American Thyroid Association; Endocrine Society 2005 Consensus Statement \#1: Subclinical thyroid dysfunction: a joint statement on management from the American Association of Clinical Endocrinologists, the American Thyroid Association, and The Endocrine Society. Thyroid 15:24-28; response 32-23.

301. Kaplowitz PB 2010 Subclinical hypothyroidism in children: normal variation or sign of a failing thyroid gland? Int J Pediatr Endocrinol 2010:281453. 
302. O'Grady MJ, Cody D 2011 Subclinical hypothyroidism in childhood. Arch Dis Child 96:280-284.

303. Radetti G, Maselli M, Buzi F, Corrias A, Mussa A, Cambiaso P, Salerno M, Cappa M, Baiocchi M, Gastaldi R, Minerba L, Loche S 2012 The natural history of the normal/mild elevated TSH serum levels in children and adolescents with Hashimoto's thyroiditis and isolated hyperthyrotropinaemia: a 3-year follow-up. Clin Endocrinol 76:394-398.

304. Lazar L, Frumkin RB, Battat E, Lebenthal Y, Phillip M, Meyerovitch J 2009 Natural history of thyroid function tests over 5 years in a large pediatric cohort. J Clin Endocrinol Metab 94:1678-1682.

305. Cerbone M, Bravaccio C, Capalbo D, Polizzi M, Wasniewska M, Cioffi D, Improda N, Valenzise M, Bruzzese D, De Luca F, Salerno M 2011 Linear growth and intellectual outcome in children with long-term idiopathic subclinical hypothyroidism. Eur J Endocrinol 164:591-597.

306. Gottardi E, Egger F, Radetti G 2008 TSH and endothelial function in children. Eur J Pediatr 167:355-356.

307. Ittermann $\mathrm{T}$, Thamm M, Wallaschofski $\mathrm{H}$, Rettig R, Volzke H 2012 Serum thyroid-stimulating hormone levels are associated with blood pressure in children and adolescents. J Clin Endocrinol Metab 97:828-834.

308. Zepf FD, Vloet TD, Polier GG, Baurmann D, Bubenzer S, Helmbold K, Gaber TJ, Schmidt T, Herpertz-Dahlmann B, Wockel L 2011 No association between affective and behavioral dysregulation and parameters of thyroid function in youths. J Affect Disord 134:478-482.

309. Monzani A, Prodam F, Rapa A, Moia S, Agarla V, Bellone S, Bona G 2013 Endocrine disorders in childhood and adolescence. Natural history of subclinical hypothyroidism in children and adolescents and potential effects of replacement therapy: a review. Eur J Endocrinol 168: R1-R11.

310. Pacifico L, Anania C, Ferraro F, Andreoli GM, Chiesa C 2012 Thyroid function in childhood obesity and metabolic comorbidity. Clin Chim Acta 413:396-405.

311. Expert Panel on Integrated Guidelines for Cardiovascular Health and Risk Reduction in Children and Adolescents; National Heart, Lung, and Blood Institute 2011 Expert panel on integrated guidelines for cardiovascular health and risk reduction in children and adolescents: summary report. Pediatrics 128(Suppl 5):S213-S256.

312. Massimino M, Gandola L, Seregni E, Bongarzone I, Morosi C, Collini P 2009 Thyroid iatrogenic sequelae after the treatment of pediatric cancer. Q J Nucl Med Mol Imaging 53:526-535.

313. Subbiah S, Collins BJ, Schneider AB 2007 Factors related to the recurrence of thyroid nodules after surgery for benign radiation-related nodules. Thyroid 17:41-47.

314. Sturgess I, Thomas SH, Pennell DJ, Mitchell D, Croft DN 1989 Diurnal variation in TSH and free thyroid hormones in patients on thyroxine replacement. Acta Endocrinol (Copenh) 121:674-676.

315. Wennlund A 1986 Variation in serum levels of T3, T4, FT4 and TSH during thyroxine replacement therapy. Acta Endocrinol (Copenh) 113:47-49.

316. Biersack HJ, Hartmann F, Rodel R, Reinhardt M 2004 Long term changes in serum T4, T3, and TSH in benign thyroid disease: proof of a narrow individual variation. Nuklearmedizin 43:158-160; quiz 162-153.

317. Ain KB, Refetoff S, Fein HG, Weintraub BD 1991 Pseudomalabsorption of levothyroxine. JAMA 266:2118-2120.
318. Molines L, Fromont I, Morlet-Barla N, Nogueira JP, Valero R, Vialettes B 2007 L-thyroxine pseudomalabsorption: a factitious disease [in French]. Presse Med 36:1390-1394.

319. Mussig K, Morike K, Klein R, Strater J, Georges G, Haring HU, Schnauder G 2009 Hypothyroidism due to pseudo-malabsorption of levothyroxine-Case 12/2009 [in German]. Dtsch Med Wochenschr 134:2514.

320. Eledrisi MS, Szymajda A, Alshanti M, Urban RJ 2001 Noncompliance with medical treatment: pseudomalabsorption of levothyroxine. South Med J 94:833-836.

321. Livadariu E, Valdes-Socin H, Burlacu MC, Vulpoi C, Daly AF, Beckers A 2007 Pseudomalabsorption of thyroid hormones: case report and review of the literature. Ann Endocrinol (Paris) 68:460-463.

322. Walker JN, Shillo P, Ibbotson V, Vincent A, Karavitaki N, Weetman AP, Wass JA, Allahabadia A 2013 A thyroxine absorption test followed by weekly thyroxine administration: a method to assess non-adherence to treatment. Eur J Endocrinol 168:913-917.

323. Mendoza H, Connacher A, Srivastava R 2009 Unexplained high thyroid stimulating hormone: a "BIG" problem. BMJ Case Rep pii: bcr01.2009.1474.

324. England ML, Hershman JM 1986 Serum TSH concentration as an aid to monitoring compliance with thyroid hormone therapy in hypothyroidism. Am J Med Sci 292:264-266.

325. Crilly M 2004 Thyroxine adherence in primary hypothyroidism. Lancet 363:1558.

326. Kubota S, Fukata S, Matsuzuka F, Kuma K, Miyauchi A 2003 Successful management of a patient with pseudomalabsorption of levothyroxine. Int $\mathrm{J}$ Psychiatry Med 33:183-188.

327. Sekadde CB, Slaunwhite WR Jr, Aceto T Jr, Murray K 1974 Administration of thyroxin once a week. J Clin Endocrinol Metab 39:759-764.

328. Bernstein RS, Robbins J 1969 Intermittent therapy with Lthyroxine. N Engl J Med 281:1444-1448.

329. Rangan S, Tahrani AA, Macleod AF, Moulik PK 2007 Once weekly thyroxine treatment as a strategy to treat non-compliance. Postgrad Med J 83:e3.

330. Crilly M, Esmail A 2005 Randomised controlled trial of a hypothyroid educational booklet to improve thyroxine adherence. Br J Gen Pract 55:362-368.

331. Grebe SK, Cooke RR, Ford HC, Fagerstrom JN, Cordwell DP, Lever NA, Purdie GL, Feek CM 1997 Treatment of hypothyroidism with once weekly thyroxine. J Clin Endocrinol Metab 82:870-875.

332. Taylor J, Williams BO, Frater J, Stott DJ, Connell J 1994 Twice-weekly dosing for thyroxine replacement in elderly patients with primary hypothyroidism. J Int Med Res 22:273-277.

333. Bornschein A, Paz-Filho G, Graf H, Carvalho GA 2012 Treating primary hypothyroidism with weekly doses of levothyroxine: a randomized, single-blind, crossover study. Arq Bras Endocrinol Metabol 56:250-258.

334. Jauk B, Mikosch P, Gallowitsch HJ, Kresnik E, Molnar M, Gomez I, Lind P 2000 Unusual malabsorption of levothyroxine. Thyroid 10:93-95.

335. Hays MT 2007 Parenteral thyroxine administration. Thyroid 17:127-129.

336. Pedraza PE, Obregon MJ, Escobar-Morreale HF, del Rey FE, de Escobar GM 2006 Mechanisms of adaptation to iodine deficiency in rats: thyroid status is tissue specific. Its relevance for man. Endocrinology 147:2098-2108. 
337. Riesco G, Taurog A, Larsen PR 1976 Variations in the response of the thyroid gland of the rat to different lowiodine diets: correlation with iodine content of diet. Endocrinology 99:270-280.

338. Escobar-Morreale HF, Obregon MJ, Escobar del Rey F, Morreale de Escobar G 1995 Replacement therapy for hypothyroidism with thyroxine alone does not ensure euthyroidism in all tissues, as studied in thyroidectomized rats. J Clin Invest 96:2828-2838.

339. Christoffolete MA, Arrojo e Drigo R, Gazoni F, Tente SM, Goncalves V, Amorim BS, Larsen PR, Bianco AC, Zavacki AM 2007 Mice with impaired extrathyroidal thyroxine to $3,5,3^{\prime}$-triiodothyronine conversion maintain normal serum 3,5,3'-triiodothyronine concentrations. Endocrinology 148:954-960.

340. Galton VA, Wood ET, St Germain EA, Withrow CA, Aldrich G, St Germain GM, Clark AS, St Germain DL 2007 Thyroid hormone homeostasis and action in the type 2 deiodinase-deficient rodent brain during development. Endocrinology 148:3080-3088.

341. Galton VA, Schneider MJ, Clark AS, St Germain DL 2009 Life without thyroxine to 3,5,3'-triiodothyronine conversion: studies in mice devoid of the $5^{\prime}$-deiodinases. Endocrinology 150:2957-2963.

342. Fonseca TL, Correa-Medina M, Campos MP, Wittmann G, Werneck-de-Castro JP, Arrojo e Drigo R, Mora-Garzon M, Ueta CB, Caicedo A, Fekete C, Gereben B, Lechan RM, Bianco AC 2013 Coordination of hypothalamic and pituitary $\mathrm{T}_{3}$ production regulates $\mathrm{TSH}$ expression. $\mathrm{J}$ Clin Invest 123:1492-1500.

343. Choosing Wisely. An initiative of the ABIM Foundation. Five things physicians and patients should question. Available at: http://www.choosingwisely.org/doctor-patient-lists/ the-endocrine-society-and-american-association-of-clinicalendocrinologists/

344. Chopra IJ, Hershman JM, Hornabrook RW 1975 Serum thyroid hormone and thyrotropin levels in subjects from endemic goiter regions of New Guinea. J Clin Endocrinol Metab 40:326-333.

345. Andersen S, Bruun NH, Pedersen KM, Laurberg P 2003 Biologic variation is important for interpretation of thyroid function tests. Thyroid 13:1069-1078.

346. Russell W, Harrison RF, Smith N, Darzy K, Shalet S, Weetman AP, Ross RJ 2008 Free triiodothyronine has a distinct circadian rhythm that is delayed but parallels thyrotropin levels. J Clin Endocrinol Metab 93:2300-2306.

347. Celi FS, Zemskova M, Linderman JD, Smith S, Drinkard B, Sachdev V, Skarulis MC, Kozlosky M, Csako G, Costello R, Pucino F 2011 Metabolic effects of liothyronine therapy in hypothyroidism: a randomized, doubleblind, crossover trial of liothyronine versus levothyroxine. J Clin Endocrinol Metab 96:3466-3474.

348. Celi FS, Zemskova M, Linderman JD, Babar NI, Skarulis MC, Csako G, Wesley R, Costello R, Penzak SR, Pucino F 2010 The pharmacodynamic equivalence of levothyroxine and liothyronine: a randomized, double blind, crossover study in thyroidectomized patients. Clin Endocrinol 72:709-715.

349. Eisenberg M, Samuels M, DiStefano JJ, 3rd 2008 Extensions, validation, and clinical applications of a feedback control system simulator of the hypothalamo-pituitarythyroid axis. Thyroid 18:1071-1085.

350. Meier C, Trittibach P, Guglielmetti M, Staub JJ, Muller B 2003 Serum thyroid stimulating hormone in assessment of severity of tissue hypothyroidism in patients with overt primary thyroid failure: cross sectional survey. BMJ 326:311-312.

351. Soukhova N, Soldin OP, Soldin SJ 2004 Isotope dilution tandem mass spectrometric method for T4/T3. Clin Chim Acta 343: 185-190.

352. Wu AH, French D 2013 Implementation of liquid chromatography/mass spectrometry into the clinical laboratory. Clin Chim Acta 420:4-10.

353. Jennings PE, O'Malley BP, Griffin KE, Northover B, Rosenthal FD 1984 Relevance of increased serum thyroxine concentrations associated with normal serum triiodothyronine values in hypothyroid patients receiving thyroxine: a case for "tissue thyrotoxicosis". Br Med J (Clin Res Ed) 289:1645-1647.

354. Bindels AJ, Meinders AE 1988 The serum concentrations of T3, T4 and TSH in evaluating replacement therapy in primary hypothyroidism. Neth J Med 32:59-71.

355. Bolk N, Visser TJ, Kalsbeek A, van Domburg RT, Berghout A 2007 Effects of evening vs morning thyroxine ingestion on serum thyroid hormone profiles in hypothyroid patients. Clin Endocrinol 66:43-48.

356. Adler SM, Wartofsky L 2007 The nonthyroidal illness syndrome. Endocrinol Metab Clin North Am 36:657-672, vi.

357. Gereben B, Goncalves C, Harney JW, Larsen PR, Bianco AC 2000 Selective proteolysis of human type 2 deiodinase: a novel ubiquitin-proteasomal mediated mechanism for regulation of hormone activation. Mol Endocrinol 14:1697-1708.

358. Gereben B, Zavacki AM, Ribich S, Kim BW, Huang SA, Simonides WS, Zeold A, Bianco AC 2008 Cellular and molecular basis of deiodinase-regulated thyroid hormone signaling. Endocr Rev 29:898-938.

359. Visser WE, Friesema EC, Visser TJ 2011 Minireview: thyroid hormone transporters: the knowns and the unknowns. Mol Endocrinol 25:1-14.

360. Larsen PR, Silva JE, Kaplan MM 1981 Relationships between circulating and intracellular thyroid hormones: physiological and clinical implications. Endocr Rev 2:87102.

361. Escobar-Morreale HF, del Rey FE, Obregon MJ, de Escobar GM 1996 Only the combined treatment with thyroxine and triiodothyronine ensures euthyroidism in all tissues of the thyroidectomized rat. Endocrinology 137: 2490-2502.

362. Watanabe M, Houten SM, Mataki C, Christoffolete MA, Kim BW, Sato H, Messaddeq N, Harney JW, Ezaki O, Kodama T, Schoonjans K, Bianco AC, Auwerx J 2006 Bile acids induce energy expenditure by promoting intracellular thyroid hormone activation. Nature 439:484489.

363. Simonides WS, Mulcahey MA, Redout EM, Muller A, Zuidwijk MJ, Visser TJ, Wassen FW, Crescenzi A, daSilva WS, Harney J, Engel FB, Obregon MJ, Larsen PR, Bianco AC, Huang SA 2008 Hypoxia-inducible factor induces local thyroid hormone inactivation during hypoxicischemic disease in rats. J Clin Invest 118:975-983.

364. Spencer CA, LoPresti JS, Patel A, Guttler RB, Eigen A, Shen D, Gray D, Nicoloff JT 1990 Applications of a new chemiluminometric thyrotropin assay to subnormal measurement. J Clin Endocrinol Metab 70:453-460.

365. Hoermann R, Eckl W, Hoermann C, Larisch R 2010 Complex relationship between free thyroxine and TSH in 
the regulation of thyroid function. Eur $\mathrm{J}$ Endocrinol 162:1123-1129.

366. Over R, Mannan S, Nsouli-Maktabi H, Burman KD, Jonklaas J 2010 Age and the thyrotropin response to hypothyroxinemia. J Clin Endocrinol Metab 95:3675-3683.

367. Hadlow NC, Rothacker KM, Wardrop R, Brown SJ, Lim EM, Walsh JP 2013 The relationship between TSH and free $\mathrm{T}(4)$ in a large population is complex and nonlinear and differs by age and sex. J Clin Endocrinol Metab 98:2936-2943.

368. Baloch Z, Carayon P, Conte-Devolx B, Demers LM, Feldt-Rasmussen U, Henry JF, LiVosli VA, Niccoli-Sire P, John R, Ruf J, Smyth PP, Spencer CA, Stockigt JR 2003 Laboratory medicine practice guidelines. Laboratory support for the diagnosis and monitoring of thyroid disease. Thyroid 13:3-126.

369. Andersen S, Pedersen KM, Bruun NH, Laurberg P 2002 Narrow individual variations in serum $\mathrm{T}(4)$ and $\mathrm{T}(3)$ in normal subjects: a clue to the understanding of subclinical thyroid disease. J Clin Endocrinol Metab 87:1068-1072.

370. Ridgway EC, Cooper DS, Walker H, Daniels GH, Chin WW, Myers G, Maloof F 1980 Therapy of primary hypothyroidism with L-triiodothyronine: discordant cardiac and pituitary responses. Clin Endocrinol 13:479-488.

371. Spencer CA, Lum SM, Wilber JF, Kaptein EM, Nicoloff JT 1983 Dynamics of serum thyrotropin and thyroid hormone changes in fasting. J Clin Endocrinol Metab 56:883-888.

372. Reed HL, Silverman ED, Shakir KM, Dons R, Burman KD, O'Brian JT 1990 Changes in serum triiodothyronine (T3) kinetics after prolonged Antarctic residence: the polar $\mathrm{T}_{3}$ syndrome. J Clin Endocrinol Metab 70:965-974.

373. Yavuz S, Linderman JD, Smith S, Zhao X, Pucino F, Celi FS 2013 The dynamic pituitary response to escalatingdose TRH stimulation test in hypothyroid patients treated with liothyronine or levothyroxine replacement therapy. J Clin Endocrinol Metab 98:E862-866.

374. Hoermann R, Midgley JE, Larisch R, Dietrich JW 2013 Is pituitary TSH an adequate measure of thyroid hormonecontrolled homoeostasis during thyroxine treatment? Eur J Endocrinol 168:271-280.

375. Midgley JE, Hoermann R, Larisch R, Dietrich JW 2013 Physiological states and functional relation between thyrotropin and free thyroxine in thyroid health and disease: in vivo and in silico data suggest a hierarchical model. $\mathrm{J}$ Clin Pathol 66:335-342.

376. Bochukova E, Schoenmakers N, Agostini M, Schoenmakers E, Rajanayagam O, Keogh JM, Henning E, Reinemund J, Gevers E, Sarri M, Downes K, Offiah A, Albanese A, Halsall D, Schwabe JW, Bain M, Lindley K, Muntoni F, Vargha-Khadem F, Dattani M, Farooqi IS, Gurnell M, Chatterjee K 2012 A mutation in the thyroid hormone receptor alpha gene. N Engl J Med 366: $243-249$.

377. van Mullem A, van Heerebeek R, Chrysis D, Visser E, Medici M, Andrikoula M, Tsatsoulis A, Peeters R, Visser TJ 2012 Clinical phenotype and mutant TRalpha1. N Engl J Med 366:1451-1453.

378. Refetoff S, Dumitrescu AM 2007 Syndromes of reduced sensitivity to thyroid hormone: genetic defects in hormone receptors, cell transporters and deiodination. Best Pract Res Clin Endocrinol Metab 21:277-305.

379. Visser WE, van Mullem AA, Visser TJ, Peeters RP 2013 Different causes of reduced sensitivity to thyroid hor- mone: diagnosis and clinical management. Clin Endocrinol 79:595-605.

380. Brucker-Davis F, Skarulis MC, Grace MB, Benichou J, Hauser P, Wiggs E, Weintraub BD 1995 Genetic and clinical features of 42 kindreds with resistance to thyroid hormone. The National Institutes of Health Prospective Study. Ann Intern Med 123:572-583.

381. Boeving A, Paz-Filho G, Radominski RB, Graf H, Amaral de Carvalho G 2011 Low-normal or high-normal thyrotropin target levels during treatment of hypothyroidism: a prospective, comparative study. Thyroid 21:355-360.

382. Weinreb JT, Yang Y, Braunstein GD 2011 Do patients gain weight after thyroidectomy for thyroid cancer? Thyroid 21:1339-1342.

383. Jonklaas J, Nsouli-Maktabi H 2011 Weight changes in euthyroid patients undergoing thyroidectomy. Thyroid 21: 1343-1351.

384. Rodriguez T, Lavis VR, Meininger JC, Kapadia AS, Stafford LF 2005 Substitution of liothyronine at a 1:5 ratio for a portion of levothyroxine: effect on fatigue, symptoms of depression, and working memory versus treatment with levothyroxine alone. Endocr Pract 11:223-233.

385. Walsh JP, Shiels L, Lim EM, Bhagat CI, Ward LC, Stuckey BG, Dhaliwal SS, Chew GT, Bhagat MC, Cussons AJ 2003 Combined thyroxine/liothyronine treatment does not improve well-being, quality of life, or cognitive function compared to thyroxine alone: a randomized controlled trial in patients with primary hypothyroidism. J Clin Endocrinol Metab 88:4543-4550.

386. Appelhof BC, Fliers E, Wekking EM, Schene AH, Huyser J, Tijssen JG, Endert E, van Weert HC, Wiersinga WM 2005 Combined therapy with levothyroxine and liothyronine in two ratios, compared with levothyroxine monotherapy in primary hypothyroidism: a double-blind, randomized, controlled clinical trial. J Clin Endocrinol Metab 90:26662674.

387. Bunevicius R, Kazanavicius G, Zalinkevicius R, Prange AJ Jr 1999 Effects of thyroxine as compared with thyroxine plus triiodothyronine in patients with hypothyroidism. N Engl J Med 340:424-429.

388. Sawka AM, Gerstein HC, Marriott MJ, MacQueen GM, Joffe RT 2003 Does a combination regimen of thyroxine (T4) and 3,5,3'-triiodothyronine improve depressive symptoms better than $\mathrm{T} 4$ alone in patients with hypothyroidism? Results of a double-blind, randomized, controlled trial. J Clin Endocrinol Metab 88:4551-4555.

389. Grozinsky-Glasberg S, Fraser A, Nahshoni E, Weizman A, Leibovici L 2006 Thyroxine-triiodothyronine combination therapy versus thyroxine monotherapy for clinical hypothyroidism: meta-analysis of randomized controlled trials. J Clin Endocrinol Metab 91:2592-2599.

390. Panicker V, Saravanan P, Vaidya B, Evans J, Hattersley AT, Frayling TM, Dayan CM 2009 Common variation in the DIO2 gene predicts baseline psychological well-being and response to combination thyroxine plus triiodothyronine therapy in hypothyroid patients. J Clin Endocrinol Metab 94:1623-1629.

391. Lania A, Persani L, Beck-Peccoz P 2008 Central hypothyroidism. Pituitary 11:181-186.

392. Beck-Peccoz P 2011 Treatment of central hypothyroidism. Clin Endocrinol 74:671-672.

393. Ferretti E, Persani L, Jaffrain-Rea ML, Giambona S, Tamburrano G, Beck-Peccoz P 1999 Evaluation of the adequacy of levothyroxine replacement therapy in patients 
with central hypothyroidism. J Clin Endocrinol Metab 84:924-929.

394. Alexopoulou O, Beguin C, De Nayer P, Maiter D 2004 Clinical and hormonal characteristics of central hypothyroidism at diagnosis and during follow-up in adult patients. Eur J Endocrinol 150:1-8.

395. Koulouri O, Auldin MA, Agarwal R, Kieffer V, Robertson C, Falconer Smith J, Levy MJ, Howlett TA 2011 Diagnosis and treatment of hypothyroidism in TSH deficiency compared to primary thyroid disease: pituitary patients are at risk of under-replacement with levothyroxine. Clin Endocrinol 74:744-749.

396. Shimon I, Cohen O, Lubetsky A, Olchovsky D 2002 Thyrotropin suppression by thyroid hormone replacement is correlated with thyroxine level normalization in central hypothyroidism. Thyroid 12:823-827.

397. Iverson JF, Mariash CN 2008 Optimal free thyroxine levels for thyroid hormone replacement in hypothyroidism. Endocr Pract 14:550-555.

398. Carrozza V, Csako G, Yanovski JA, Skarulis MC, Nieman L, Wesley R, Pucino F 1999 Levothyroxine replacement therapy in central hypothyroidism: a practice report. Pharmacotherapy 19:349-355.

399. Klose M, Marina D, Hartoft-Nielsen ML, Klefter O, Gavan V, Hilsted L, Rasmussen AK, Feldt-Rasmussen U 2013 Central hypothyroidism and its replacement have a significant influence on cardiovascular risk factors in adult hypopituitary patients. J Clin Endocrinol Metab 98:3802-3810.

400. Agha A, Walker D, Perry L, Drake WM, Chew SL, Jenkins PJ, Grossman AB, Monson JP 2007 Unmasking of central hypothyroidism following growth hormone replacement in adult hypopituitary patients. Clin Endocrinol 66:72-77.

401. Razvi S, McMillan CV, Weaver JU 2005 Instruments used in measuring symptoms, health status and quality of life in hypothyroidism: a systematic qualitative review. Clin Endocrinol 63:617-624.

402. Ware JE Jr, Sherbourne CD 1992 The MOS 36-item shortform health survey (SF-36). I. Conceptual framework and item selection. Med Care 30:473-483.

403. Hunt SM, McEwen J, McKenna SP 1985 Measuring health status: a new tool for clinicians and epidemiologists. J R Coll Gen Pract 35:185-188.

404. Goldberg DP, Hillier VF 1979 A scaled version of the General Health Questionnaire. Psychol Med 9:139-145.

405. Zigmond AS, Snaith RP 1983 The hospital anxiety and depression scale. Acta Psychiatr Scand 67:361-370.

406. Jaeschke R, Guyatt G, Cook D, Harper S, Gerstein HC 1994 Spectrum of quality of life impairment in hypothyroidism. Qual Life Res 3:323-327.

407. McMillan CV, Bradley C, Woodcock A, Razvi S, Weaver JU 2004 Design of new questionnaires to measure quality of life and treatment satisfaction in hypothyroidism. Thyroid 14:916-925.

408. McMillan C, Bradley C, Razvi S, Weaver J 2008 Evaluation of new measures of the impact of hypothyroidism on quality of life and symptoms: the ThyDQoL and ThySRQ. Value Health 11:285-294.

409. Watt T, Hegedus L, Groenvold M, Bjorner JB, Rasmussen AK, Bonnema SJ, Feldt-Rasmussen U 2010 Validity and reliability of the novel thyroid-specific quality of life questionnaire, ThyPRO. Eur J Endocrinol 162:161-167.

410. McMillan C, Bradley C, Razvi S, Weaver J 2006 Psychometric evaluation of a new questionnaire measuring treatment satisfaction in hypothyroidism: the ThyTSQ. Value Health 9:132-139.

411. Eskelinen SI, Vahlberg TJ, Isoaho RE, Lopponen MK, Kivela SL, Irjala KM 2007 Associations of thyroidstimulating hormone and free thyroxine concentrations with health and life satisfaction in elderly adults. Endocr Pract 13:451-457.

412. van de Ven AC, Netea-Maier RT, de Vegt F, Ross HA, Sweep FC, Kiemeney LA, Hermus AR, den Heijer M 2012 Is there a relationship between fatigue perception and the serum levels of thyrotropin and free thyroxine in euthyroid subjects? Thyroid 22:1236-1243.

413. Wekking EM, Appelhof BC, Fliers E, Schene AH, Huyser J, Tijssen JG, Wiersinga WM 2005 Cognitive functioning and well-being in euthyroid patients on thyroxine replacement therapy for primary hypothyroidism. Eur J Endocrinol 153:747-753.

414. O'Reilly DS 2010 Thyroid hormone replacement: an iatrogenic problem. Int J Clin Pract 64:991-994.

415. Carr D, McLeod DT, Parry G, Thornes HM 1988 Fine adjustment of thyroxine replacement dosage: comparison of the thyrotrophin releasing hormone test using a sensitive thyrotrophin assay with measurement of free thyroid hormones and clinical assessment. Clin Endocrinol 28: 325-333.

416. Ott J, Promberger R, Kober F, Neuhold N, Tea M, Huber JC, Hermann M 2011 Hashimoto's thyroiditis affects symptom load and quality of life unrelated to hypothyroidism: a prospective case-control study in women undergoing thyroidectomy for benign goiter. Thyroid 21:161-167.

417. Pop VJ, Maartens LH, Leusink G, van Son MJ, Knottnerus AA, Ward AM, Metcalfe R, Weetman AP 1998 Are autoimmune thyroid dysfunction and depression related? J Clin Endocrinol Metab 83:3194-3197.

418. Bould H, Panicker V, Kessler D, Durant C, Lewis G, Dayan C, Evans J 2012 Investigation of thyroid dysfunction is more likely in patients with high psychological morbidity. Fam Pract 29:163-167.

419. Burch HB, Burman KD, Cooper DS, Hennessey JV 2014 A 2013 Survey of clinical practice patterns in the management of primary hypothyroidism. J Clin Endocrinol Metab 99:2077-2085.

420. Biondi B, Wartofsky L 2014 Treatment with thyroid hormone. Endocr Rev 35:433-512.

421. Pollock MA, Sturrock A, Marshall K, Davidson KM, Kelly CJ, McMahon AD, McLaren EH 2001 Thyroxine treatment in patients with symptoms of hypothyroidism but thyroid function tests within the reference range: randomised double blind placebo controlled crossover trial. BMJ 323:891-895.

422. Dubois S, Abraham P, Rohmer V, Rodien P, Audran M, Dumas JF, Ritz P 2008 Thyroxine therapy in euthyroid patients does not affect body composition or muscular function. Thyroid 18:13-19.

423. Maes M, Meltzer HY, Cosyns P, Suy E, Schotte C 1993 An evaluation of basal hypothalamic-pituitary-thyroid axis function in depression: results of a large-scaled and controlled study. Psychoneuroendocrinology 18:607-620.

424. Engum A, Bjoro T, Mykletun A, Dahl AA 2002 An association between depression, anxiety and thyroid function-a clinical fact or an artefact? Acta Psychiatr Scand 106:27-34.

425. Brouwer JP, Appelhof BC, Hoogendijk WJ, Huyser J, Endert E, Zuketto C, Schene AH, Tijssen JG, Van Dyck 
R, Wiersinga WM, Fliers E 2005 Thyroid and adrenal axis in major depression: a controlled study in outpatients. Eur J Endocrinol 152:185-191.

426. Whybrow PC, Prange AJ Jr, Treadway CR 1969 Mental changes accompanying thyroid gland dysfunction. A reappraisal using objective psychological measurement. Arch Gen Psychiatry 20:48-63.

427. Constant EL, Adam S, Seron X, Bruyer R, Seghers A, Daumerie C 2005 Anxiety and depression, attention, and executive functions in hypothyroidism. J Int Neuropsychol Soc 11:535-544.

428. Park YJ, Lee EJ, Lee YJ, Choi SH, Park JH, Lee SB, Lim S, Lee WW, Jang HC, Cho BY, Woo JI, Kim KW 2010 Subclinical hypothyroidism $(\mathrm{SCH})$ is not associated with metabolic derangement, cognitive impairment, depression or poor quality of life (QoL) in elderly subjects. Arch Gerontol Geriatr 50:e68-73.

429. Almeida OP, Alfonso H, Flicker L, Hankey G, Chubb SA, Yeap BB 2011 Thyroid hormones and depression: the Health in Men study. Am J Geriatr Psychiatry 19:763-770.

430. Jorde R, Waterloo K, Storhaug H, Nyrnes A, Sundsfjord J, Jenssen TG 2006 Neuropsychological function and symptoms in subjects with subclinical hypothyroidism and the effect of thyroxine treatment. J Clin Endocrinol Metab 91:145-153.

431. Bauer MS, Whybrow PC 1990 Rapid cycling bipolar affective disorder. II. Treatment of refractory rapid cycling with high-dose levothyroxine: a preliminary study. Arch Gen Psychiatry 47:435-440.

432. Bauer M, London ED, Rasgon N, Berman SM, Frye MA, Altshuler LL, Mandelkern MA, Bramen J, Voytek B, Woods R, Mazziotta JC, Whybrow PC 2005 Supraphysiological doses of levothyroxine alter regional cerebral metabolism and improve mood in bipolar depression. Mol Psychiatry 10:456-469.

433. Bauer M, Hellweg R, Graf KJ, Baumgartner A 1998 Treatment of refractory depression with high-dose thyroxine. Neuropsychopharmacology 18:444-455.

434. Joffe RT, Singer W 1990 A comparison of triiodothyronine and thyroxine in the potentiation of tricyclic antidepressants. Psychiatry Res 32:241-251.

435. Flegal KM, Carroll MD, Kit BK, Ogden CL 2012 Prevalence of obesity and trends in the distribution of body mass index among US adults, 1999-2010. JAMA 307: 491-497.

436. Ljungvall A, Zimmerman FJ 2012 Bigger bodies: longterm trends and disparities in obesity and body-mass index among U.S. adults, 1960-2008. Soc Sci Med 75:109-119.

437. Knudsen N, Laurberg P, Rasmussen LB, Bulow I, Perrild $\mathrm{H}$, Ovesen L, Jorgensen $\mathrm{T} 2005$ Small differences in thyroid function may be important for body mass index and the occurrence of obesity in the population. J Clin Endocrinol Metab 90:4019-4024.

438. Fox CS, Pencina MJ, D’Agostino RB, Murabito JM, Seely EW, Pearce EN, Vasan RS 2008 Relations of thyroid function to body weight: cross-sectional and longitudinal observations in a community-based sample. Arch Intern Med 168:587-592.

439. Asvold BO, Bjoro T, Vatten LJ 2009 Association of serum TSH with high body mass differs between smokers and never-smokers. J Clin Endocrinol Metab 94:5023-5027.

440. Marzullo P, Minocci A, Tagliaferri MA, Guzzaloni G, Di Blasio A, De Medici C, Aimaretti G, Liuzzi A 2010 Investigations of thyroid hormones and antibodies in obesity: leptin levels are associated with thyroid autoimmunity independent of bioanthropometric, hormonal, and weightrelated determinants. J Clin Endocrinol Metab 95:39653972.

441. Danforth E Jr, Burger AG, Wimpfheimer C 1978 Nutritionally-induced alterations in thyroid hormone metabolism and thermogenesis. Experientia Suppl 32:213-217.

442. Chikunguwo S, Brethauer S, Nirujogi V, Pitt T, Udomsawaengsup S, Chand B, Schauer P 2007 Influence of obesity and surgical weight loss on thyroid hormone levels. Surg Obes Relat Dis 3:631-635; discussion 635636.

443. Karmisholt J, Andersen S, Laurberg P 2011 Weight loss after therapy of hypothyroidism is mainly caused by excretion of excess body water associated with myxoedema. J Clin Endocrinol Metab 96:E99-E103.

444. Kong WM, Sheikh MH, Lumb PJ, Naoumova RP, Freedman DB, Crook M, Dore CJ, Finer N 2002 A 6month randomized trial of thyroxine treatment in women with mild subclinical hypothyroidism. Am J Med 112: 348-354.

445. Rivlin RS 1975 Therapy of obesity with hormones. N Engl J Med 292:26-29.

446. Gardner DF, Kaplan MM, Stanley CA, Utiger RD 1979 Effect of tri-iodothyronine replacement on the metabolic and pituitary responses to starvation. N Engl J Med 300: 579-584.

447. Kaptein EM, Beale E, Chan LS 2009 Thyroid hormone therapy for obesity and nonthyroidal illnesses: a systematic review. J Clin Endocrinol Metab 94:3663-3675.

448. Bagnasco M, Minciullo PL, Saraceno GS, Gangemi S, Benvenga S 2011 Urticaria and thyroid autoimmunity. Thyroid 21:401-410.

449. Monge C, Demarco P, Burman KD, Wartofsky L 2007 Autoimmune thyroid disease and chronic urticaria. Clin Endocrinol 67:473-475.

450. Magen E, Mishal J 2012 The effect of L-thyroxine treatment on chronic idiopathic urticaria and autoimmune thyroiditis. Int J Dermatol 51:94-97.

451. Kiyici S, Gul OO, Baskan EB, Hacioglu S, Budak F, Erturk E, Imamoglu S 2010 Effect of levothyroxine treatment on clinical symptoms and serum cytokine levels in euthyroid patients with chronic idiopathic urticaria and thyroid autoimmunity. Clin Exp Dermatol 35:603-607.

452. Cohen JH, 3rd, Ingbar SH, Braverman LE 1989 Thyrotoxicosis due to ingestion of excess thyroid hormone. Endocr Rev 10:113-124.

453. Bahn RS, Burch HB, Cooper DS, Garber JR, Greenlee MC, Klein I, Laurberg P, McDougall IR, Montori VM, Rivkees SA, Ross DS, Sosa JA, Stan MN 2011 Hyperthyroidism and other causes of thyrotoxicosis: management guidelines of the American Thyroid Association and American Association of Clinical Endocrinologists. Endocr Pract 17:456-520.

454. Ohye H, Fukata S, Kanoh M, Kubota S, Kuma K, Miyauchi A, Sugawara M 2005 Thyrotoxicosis caused by weight-reducing herbal medicines. Arch Intern Med 165: 831-834.

455. Rosenthal MS 2007 The impaired hypothyroid patient: ethical considerations and obligations. Thyroid 17:12611267.

456. Rosenthal MS, Angelos P, Cooper DS, Fassler C, Finder SG, Hays MT, Tendler B, Braunstein GD, American Thyroid Association Ethics Advisory C 2013 Clinical and 
professional ethics guidelines for the practice of thyroidology. Thyroid 23:1203-1210.

457. Rosenthal MS 2014 Ethical issues in the management of thyroid disease. Endocrinol Metab Clin North Am 43:545-564.

458. Lantos J, Matlock AM, Wendler D 2011 Clinician integrity and limits to patient autonomy. JAMA 305:495-499.

459. Loewy EH 2005 In defense of paternalism. Theor Med Bioeth 26:445-468.

460. Tauber AI 2003 Sick autonomy. Perspect Biol Med 46:484-495.

461. Lin R 2011 Michael Kamrava's medical license revoked. Los Angeles Times, June 1.

462. Ryan H, Kim V 2011 Conrad Murray convicted in Michael Jackson's death. Los Angeles Times, November 29.

463. Rosenthal MS 2011 The Suleman octuplet case and egregious ethical breaches. Womens Health Issues 21:98.

464. Pellegrino ED, Relman AS 1999 Professional medical associations: ethical and practical guidelines. JAMA 282: 984-986.

465. Pellegrino ED 2002 Professionalism, profession and the virtues of the good physician. Mt Sinai J Med 69:378384.

466. Cheng SY, Leonard JL, Davis PJ 2010 Molecular aspects of thyroid hormone actions. Endocr Rev 31:139-170.

467. Lanni A, Moreno M, Cioffi M, Goglia F 1992 Effect of 3,3'-diiodothyronine and 3,5-diiodothyronine on rat liver oxidative capacity. Mol Cell Endocrinol 86:143-148.

468. Cioffi F, Senese R, Lanni A, Goglia F 2013 Thyroid hormones and mitochondria: With a brief look at derivatives and analogues. Mol Cell Endocrinol 379:51-61.

469. Davis PJ, Zhou M, Davis FB, Lansing L, Mousa SA, Lin HY 2010 Mini-review: cell surface receptor for thyroid hormone and nongenomic regulation of ion fluxes in excitable cells. Physiol Behav 99:237-239.

470. Pinna G, Brodel O, Visser T, Jeitner A, Grau H, Eravci M, Meinhold H, Baumgartner A 2002 Concentrations of seven iodothyronine metabolites in brain regions and the liver of the adult rat. Endocrinology 143:1789-1800.

471. Pinna G, Hiedra L, Meinhold H, Eravci M, Prengel H, Brodel O, Graf KJ, Stoltenburg-Didinger G, Bauer M, Baumgartner A 1998 3,3'-Diiodothyronine concentrations in the sera of patients with nonthyroidal illnesses and brain tumors and of healthy subjects during acute stress. J Clin Endocrinol Metab 83:3071-3077.

472. Piehl S, Hoefig CS, Scanlan TS, Kohrle J 2011 Thyronamines_-past, present, and future. Endocr Rev 32:64-80.

473. LeBoff MS, Kaplan MM, Silva JE, Larsen PR 1982 Bioavailability of thyroid hormones from oral replacement preparations. Metabolism 31:900-905.

474. Jackson IM, Cobb WE 1978 Why does anyone still use desiccated thyroid USP? Am J Med 64:284-288.

475. Lev-Ran A 1983 Part-of-the-day hypertriiodothyroninemia caused by desiccated thyroid. JAMA 250:2790-2791.

476. Penny R, Frasier SD 1980 Elevated serum concentrations of triiodothyronine in hypothyroid patients. Values for patients receiving USP thyroid. Am J Dis Child 134:1618.

477. Surks MI, Schadlow AR, Oppenheimer JH 1972 A new radioimmunoassay for plasma L-triiodothyronine: measurements in thyroid disease and in patients maintained on hormonal replacement. J Clin Invest 51:3104-3113.

478. Smith SR 1984 Desiccated thyroid preparations. Obsolete therapy. Arch Intern Med 144:926-927.
479. Gaby AR 2004 Sub-laboratory hypothyroidism and the empirical use of Armour thyroid. Alt Med Rev 9:157-179.

480. Hoang TD, Olsen CH, Mai VQ, Clyde PW, Shakir MK 2013 Desiccated thyroid extract compared with levothyroxine in the treatment of hypothyroidism: a randomized, double-blind, crossover study. J Clin Endocrinol Metab 98:1982-1990.

481. Outcomes Research. Agency for Healthcare Research and Quality. Online publication \# 00-P011. Available at: http:// archive.ahrq.gov/research/findings/factsheets/outcomes/ outfact/outcomes-and-research.html

482. Clancy CM, Eisenberg JM 1998 Outcomes research: measuring the end results of health care. Science 282: 245-246.

483. Henrichs J, Ghassabian A, Peeters RP, Tiemeier H 2013 Maternal hypothyroxinemia and effects on cognitive functioning in childhood: how and why? Clin Endocrinol 79:152-162.

484. Hansen PS, Brix TH, Sorensen TI, Kyvik KO, Hegedus L 2004 Major genetic influence on the regulation of the pituitary-thyroid axis: a study of healthy Danish twins. J Clin Endocrinol Metab 89:1181-1187.

485. Samollow PB, Perez G, Kammerer CM, Finegold D, Zwartjes PW, Havill LM, Comuzzie AG, Mahaney MC, Goring HH, Blangero J, Foley TP, Barmada MM 2004 Genetic and environmental influences on thyroid hormone variation in Mexican Americans. J Clin Endocrinol Metab 89:3276-3284.

486. Friesema EC, Grueters A, Biebermann H, Krude H, von Moers A, Reeser M, Barrett TG, Mancilla EE, Svensson J, Kester MH, Kuiper GG, Balkassmi S, Uitterlinden AG, Koehrle J, Rodien P, Halestrap AP, Visser TJ 2004 Association between mutations in a thyroid hormone transporter and severe X-linked psychomotor retardation. Lancet 364:1435-1437.

487. Dumitrescu AM, Liao XH, Abdullah MS, Lado-Abeal J, Majed FA, Moeller LC, Boran G, Schomburg L, Weiss RE, Refetoff S 2005 Mutations in SECISBP2 result in abnormal thyroid hormone metabolism. Nat Genet 37: 1247-1252.

488. Ralston SH, Uitterlinden AG 2010 Genetics of osteoporosis. Endocr Rev 31:629-662.

489. Porcu E, Medici M, Pistis G, Volpato CB, Wilson SG, Cappola AR, Bos SD, Deelen J, den Heijer M, Freathy RM, Lahti J, Liu C, Lopez LM, Nolte IM, O'Connell JR, Tanaka T, Trompet S, Arnold A, Bandinelli S, Beekman M, Bohringer S, Brown SJ, Buckley BM, Camaschella C, de Craen AJ, Davies G, de Visser MC, Ford I, Forsen T, Frayling TM, Fugazzola L, Gogele M, Hattersley AT, Hermus AR, Hofman A, Houwing-Duistermaat JJ, Jensen RA, Kajantie E, Kloppenburg M, Lim EM, Masciullo C, Mariotti S, Minelli C, Mitchell BD, Nagaraja R, NeteaMaier RT, Palotie A, Persani L, Piras MG, Psaty BM, Raikkonen K, Richards JB, Rivadeneira F, Sala C, Sabra MM, Sattar N, Shields BM, Soranzo N, Starr JM, Stott DJ, Sweep FC, Usala G, van der Klauw MM, van Heemst D, van Mullem A, Vermeulen SH, Visser WE, Walsh JP, Westendorp RG, Widen E, Zhai G, Cucca F, Deary IJ, Eriksson JG, Ferrucci L, Fox CS, Jukema JW, Kiemeney LA, Pramstaller PP, Schlessinger D, Shuldiner AR, Slagboom EP, Uitterlinden AG, Vaidya B, Visser TJ, Wolffenbuttel BH, Meulenbelt I, Rotter JI, Spector TD, Hicks AA, Toniolo D, Sanna S, Peeters RP, Naitza S 2013 A meta-analysis of thyroid-related traits reveals novel loci 
and gender-specific differences in the regulation of thyroid function. PLoS Genet 9:e1003266.

490. Medici M, van der Deure WM, Verbiest M, Vermeulen SH, Hansen PS, Kiemeney LA, Hermus AR, Breteler MM, Hofman A, Hegedus L, Kyvik KO, den Heijer M, Uitterlinden AG, Visser TJ, Peeters RP 2011 A large-scale association analysis of 68 thyroid hormone pathway genes with serum TSH and FT4 levels. Eur J Endocrinol 164:781-788.

491. Mentuccia D, Proietti-Pannunzi L, Tanner K, Bacci V, Pollin TI, Poehlman ET, Shuldiner AR, Celi FS 2002 Association between a novel variant of the human type 2 deiodinase gene Thr92Ala and insulin resistance: evidence of interaction with the Trp64Arg variant of the beta-3adrenergic receptor. Diabetes 51:880-883.

492. Dora JM, Machado WE, Rheinheimer J, Crispim D, Maia AL 2010 Association of the type 2 deiodinase Thr92Ala polymorphism with type 2 diabetes: case-control study and meta-analysis. Eur J Endocrinol 163:427-434.

493. Canani LH, Capp C, Dora JM, Meyer EL, Wagner MS, Harney JW, Larsen PR, Gross JL, Bianco AC, Maia AL 2005 The type 2 deiodinase A/G (Thr92Ala) polymorphism is associated with decreased enzyme velocity and increased insulin resistance in patients with type 2 diabetes mellitus. J Clin Endocrinol Metab 90:3472-3478.

494. Peeters RP, van Toor H, Klootwijk W, de Rijke YB, Kuiper GG, Uitterlinden AG, Visser TJ 2003 Polymorphisms in thyroid hormone pathway genes are associated with plasma TSH and iodothyronine levels in healthy subjects. J Clin Endocrinol Metab 88:2880-2888.

495. Peeters RP, van den Beld AW, Attalki H, Toor H, de Rijke YB, Kuiper GG, Lamberts SW, Janssen JA, Uitterlinden AG, Visser TJ 2005 A new polymorphism in the type II deiodinase gene is associated with circulating thyroid hormone parameters. Am J Physiol Endocrinol Metab 289:E75-81.

496. Coppotelli G, Summers A, Chidakel A, Ross JM, Celi FS 2006 Functional characterization of the 258 A/G (D2ORFa-Gly3Asp) human type-2 deiodinase polymorphism: a naturally occurring variant increases the enzymatic activity by removing a putative repressor site in the $5^{\prime}$ UTR of the gene. Thyroid 16:625-632.

497. Butler PW, Smith SM, Linderman JD, Brychta RJ, Alberobello AT, Dubaz OM, Luzon JA, Skarulis MC, Cochran CS, Wesley RA, Pucino F, Celi FS 2010 The Thr92Ala $5^{\prime}$ type 2 deiodinase gene polymorphism is associated with a delayed triiodothyronine secretion in response to the thyrotropin-releasing hormone-stimulation test: a pharmacogenomic study. Thyroid 20:1407-1412.

498. Peltsverger MY, Butler PW, Alberobello AT, Smith S, Guevara Y, Dubaz OM, Luzon JA, Linderman J, Celi FS 2012 The $-258 \mathrm{~A} / \mathrm{G}$ (SNP rs12885300) polymorphism of the human type 2 deiodinase gene is associated with a shift in the pattern of secretion of thyroid hormones following a TRH-induced acute rise in TSH. Eur J Endocrinol 166:839-845.

499. Peeters RP, van der Deure WM, Visser TJ 2006 Genetic variation in thyroid hormone pathway genes; polymorphisms in the TSH receptor and the iodothyronine deiodinases. Eur J Endocrinol 155:655-662.

500. Dumitrescu AM, Liao XH, Best TB, Brockmann K, Refetoff S 2004 A novel syndrome combining thyroid and neurological abnormalities is associated with mutations in a monocarboxylate transporter gene. Am J Hum Genet 74:168-175.
501. Verge CF, Konrad D, Cohen M, Di Cosmo C, Dumitrescu AM, Marcinkowski T, Hameed S, Hamilton J, Weiss RE, Refetoff S 2012 Diiodothyropropionic acid (DITPA) in the treatment of MCT8 deficiency. J Clin Endocrinol Metab 97:4515-4523.

502. de Jong FJ, Peeters RP, den Heijer T, van der Deure WM, Hofman A, Uitterlinden AG, Visser TJ, Breteler MM 2007 The association of polymorphisms in the type 1 and 2 deiodinase genes with circulating thyroid hormone parameters and atrophy of the medial temporal lobe. J Clin Endocrinol Metab 92:636-640.

503. van der Deure WM, Hansen PS, Peeters RP, Uitterlinden AG, Fenger M, Kyvik KO, Hegedus L, Visser TJ 2009 The effect of genetic variation in the type 1 deiodinase gene on the interindividual variation in serum thyroid hormone levels: an investigation in healthy Danish twins. Clin Endocrinol 70:954-960.

504. Panicker V, Cluett C, Shields B, Murray A, Parnell KS, Perry JR, Weedon MN, Singleton A, Hernandez D, Evans J, Durant C, Ferrucci L, Melzer D, Saravanan P, Visser TJ, Ceresini G, Hattersley AT, Vaidya B, Dayan CM, Frayling TM 2008 A common variation in deiodinase 1 gene DIO1 is associated with the relative levels of free thyroxine and triiodothyronine. J Clin Endocrinol Metab 93:3075-3081.

505. Hoftijzer HC, Heemstra KA, Visser TJ, le Cessie S, Peeters RP, Corssmit EP, Smit JW 2011 The type 2 deiodinase ORFa-Gly3Asp polymorphism (rs12885300) influences the set point of the hypothalamus-pituitary-thyroid axis in patients treated for differentiated thyroid carcinoma. J Clin Endocrinol Metab 96:E1527-1533.

506. Gudmundsson J, Sulem P, Gudbjartsson DF, Jonasson JG, Masson G, He H, Jonasdottir A, Sigurdsson A, Stacey SN, Johannsdottir H, Helgadottir HT, Li W, Nagy R, Ringel MD, Kloos RT, de Visser MC, Plantinga TS, den Heijer M, Aguillo E, Panadero A, Prats E, Garcia-Castano A, De Juan A, Rivera F, Walters GB, Bjarnason H, Tryggvadottir L, Eyjolfsson GI, Bjornsdottir US, Holm H, Olafsson I, Kristjansson K, Kristvinsson H, Magnusson OT, Thorleifsson G, Gulcher JR, Kong A, Kiemeney LA, Jonsson T, Hjartarson H, Mayordomo JI, Netea-Maier RT, de la Chapelle A, Hrafnkelsson J, Thorsteinsdottir U, Rafnar T, Stefansson K 2012 Discovery of common variants associated with low TSH levels and thyroid cancer risk. Nat Genet 44:319-322.

507. Guo TW, Zhang FC, Yang MS, Gao XC, Bian L, Duan SW, Zheng ZJ, Gao JJ, Wang H, Li RL, Feng GY, St Clair D, He L 2004 Positive association of the DIO2 (deiodinase type 2) gene with mental retardation in the iodinedeficient areas of China. J Med Genet 41:585-590.

508. Gumieniak O, Perlstein TS, Williams JS, Hopkins PN, Brown NJ, Raby BA, Williams GH 2007 Ala92 type 2 deiodinase allele increases risk for the development of hypertension. Hypertension 49:461-466.

509. Meulenbelt I, Min JL, Bos S, Riyazi N, HouwingDuistermaat JJ, van der Wijk HJ, Kroon HM, Nakajima M, Ikegawa S, Uitterlinden AG, van Meurs JB, van der Deure WM, Visser TJ, Seymour AB, Lakenberg N, van der Breggen R, Kremer D, van Duijn CM, Kloppenburg M, Loughlin J, Slagboom PE 2008 Identification of DIO2 as a new susceptibility locus for symptomatic osteoarthritis. Hum Mol Genet 17:1867-1875.

510. Heemstra KA, Hoftijzer H, van der Deure WM, Peeters RP, Hamdy NA, Pereira A, Corssmit EP, Romijn JA, 
Visser TJ, Smit JW 2010 The type 2 deiodinase Thr92Ala polymorphism is associated with increased bone turnover and decreased femoral neck bone mineral density. J Bone Miner Res 25:1385-1391.

511. Ma SF, Xie L, Pino-Yanes M, Sammani S, Wade MS, Letsiou E, Siegler J, Wang T, Infusino G, Kittles RA, Flores C, Zhou T, Prabhakar BS, Moreno-Vinasco L, Villar J, Jacobson JR, Dudek SM, Garcia JG 2011 Type 2 deiodinase and host responses of sepsis and acute lung injury. Am J Respir Cell Mol Biol 45:1203-1211.

512. van der Deure WM, Peeters RP, Uitterlinden AG, Hofman A, Breteler MM, Witteman J, Visser TJ 2009 Impact of thyroid function and polymorphisms in the type 2 deiodinase on blood pressure: the Rotterdam Study and the Rotterdam Scan Study. Clin Endocrinol 71:137-144.

513. Roef GL, Rietzschel ER, De Meyer T, Bekaert S, De Buyzere ML, Van Daele C, Toye K, Kaufman JM, Taes YE 2013 Associations between single nucleotide polymorphisms in thyroid hormone transporter genes (MCT8, MCT10 and OATP1C1) and circulating thyroid hormones. Clin Chim Acta 425C:227-232.

514. Inada M, Kasagi K, Kurata S, Kazama Y, Takayama H, Torizuka K, Fukase M, Soma T 1975 Estimation of thyroxine and triiodothyronine distribution and of the conversion rate of thyroxine to triiodothyronine in man. J Clin Invest 55:1337-1348.

515. Silva JE, Gordon MB, Crantz FR, Leonard JL, Larsen PR 1984 Qualitative and quantitative differences in the pathways of extrathyroidal triiodothyronine generation between euthyroid and hypothyroid rats. J Clin Invest 73: 898-907.

516. Crantz FR, Silva JE, Larsen PR 1982 An analysis of the sources and quantity of 3,5,3'-triiodothyronine specifically bound to nuclear receptors in rat cerebral cortex and cerebellum. Endocrinology 110:367-375.

517. Bunevicius R, Jakubonien N, Jurkevicius R, Cernicat J, Lasas L, Prange AJ Jr 2002 Thyroxine vs thyroxine plus triiodothyronine in treatment of hypothyroidism after thyroidectomy for Graves' disease. Endocrine 18:129-133.

518. Clyde PW, Harari AE, Getka EJ, Shakir KM 2003 Combined levothyroxine plus liothyronine compared with levothyroxine alone in primary hypothyroidism: a randomized controlled trial. JAMA 290:2952-2958.

519. Escobar-Morreale HF, Botella-Carretero JI, Gomez-Bueno M, Galan JM, Barrios V, Sancho J 2005 Thyroid hormone replacement therapy in primary hypothyroidism: a randomized trial comparing L-thyroxine plus liothyronine with L-thyroxine alone. Ann Intern Med 142:412-424.

520. Fadeyev VV, Morgunova TB, Melnichenko GA, Dedov II 2010 Combined therapy with L-thyroxine and L-triiodothyronine compared to L-thyroxine alone in the treatment of primary hypothyroidism. Hormones (Athens) 9:245-252.

521. Nygaard B, Jensen EW, Kvetny J, Jarlov A, Faber J 2009 Effect of combination therapy with thyroxine (T4) and 3,5,3'-triiodothyronine versus T4 monotherapy in patients with hypothyroidism, a double-blind, randomised crossover study. Eur J Endocrinol 161:895-902.

522. Saravanan P, Simmons DJ, Greenwood R, Peters TJ, Dayan CM 2005 Partial substitution of thyroxine (T4) with tri-iodothyronine in patients on $\mathrm{T} 4$ replacement therapy: results of a large community-based randomized controlled trial. J Clin Endocrinol Metab 90:805-812.

523. Siegmund W, Spieker K, Weike AI, Giessmann T, Modess C, Dabers T, Kirsch G, Sanger E, Engel G, Hamm AO,
Nauck M, Meng W 2004 Replacement therapy with levothyroxine plus triiodothyronine (bioavailable molar ratio $14: 1$ ) is not superior to thyroxine alone to improve well-being and cognitive performance in hypothyroidism. Clin Endocrinol 60:750-757.

524. Valizadeh M, Seyyed-Majidi MR, Hajibeigloo H, Momtazi S, Musavinasab N, Hayatbakhsh MR 2009 Efficacy of combined levothyroxine and liothyronine as compared with levothyroxine monotherapy in primary hypothyroidism: a randomized controlled trial. Endocr Res 34:80-89.

525. Escobar-Morreale HF, Botella-Carretero JI, Escobar del Rey F, Morreale de Escobar G 2005 Review: treatment of hypothyroidism with combinations of levothyroxine plus liothyronine. J Clin Endocrinol Metab 90:4946-4954.

526. Joffe RT, Brimacombe M, Levitt AJ, Stagnaro-Green A 2007 Treatment of clinical hypothyroidism with thyroxine and triiodothyronine: a literature review and metaanalysis. Psychosomatics 48:379-384.

527. Ma C, Xie J, Huang X, Wang G, Wang Y, Wang X, Zuo S 2009 Thyroxine alone or thyroxine plus triiodothyronine replacement therapy for hypothyroidism. Nucl Med Commun 30:586-593.

528. Smith RN, Taylor SA, Massey JC 1970 Controlled clinical trial of combined triiodothyronine and thyroxine in the treatment of hypothyroidism. Br Med J 4:145-148.

529. Regalbuto C, Maiorana R, Alagona C, Paola RD, Cianci M, Alagona G, Sapienza S, Squatrito S, Pezzino V 2007 Effects of either LT4 monotherapy or LT4/LT3 combined therapy in patients totally thyroidectomized for thyroid cancer. Thyroid 17:323-331.

530. Beck AT, Steer RA, Brown GK 1996 Beck Depression Inventory, Second Edition Manual. The Psychological Corporation, San Antonio, TX.

531. Finlay-Jones RA, Murphy E 1979 Severity of psychiatric disorder and the 30-item general health questionnaire. Br J Psychiatry 134:609-616.

532. Goldberg D, Williams P 1988 User's guide to the General Health Questionnaire. NFER-NELSON, Berkshire, Great Britain.

533. Piper BF, Dibble SL, Dodd MJ, Weiss MC, Slaughter RE, Paul SM 1998 The revised Piper Fatigue Scale: psychometric evaluation in women with breast cancer. Oncol Nurs Forum 25:677-684.

534. Biondi B, Wartofsky L 2012 Combination treatment with T4 and T3: toward personalized replacement therapy in hypothyroidism? J Clin Endocrinol Metab 97:2256-2271.

535. Welborn TA 2013 Practice guidelines for hypothyroidism in adults: clinical issues and the controversy about combination therapy. Endocr Pract 19:1062-1064.

536. Garber JR, Cobin RH, Gharib H, Hennessey JV, Klein I, Mechanick JI, Pessah-Pollack R, Singer PA, Woeber KA, American Association of Clinical E, American Thyroid Association Taskforce on Hypothyroidism in Adults 2013 In response. Endocr Pract 19:1064-1065.

537. Wiersinga WM 2014 Paradigm shifts in thyroid hormone replacement therapies for hypothyroidism. Nat Rev Endocrinol 10:164-174.

538. McDermott MT 2012 Does combination T4 and T3 therapy make sense? Endocr Pract 18:750-757.

539. Perros P 2012 European Thyroid Association Guidelines on L-T4 + L-T3 combination for hypothyroidism: a weary step in the right direction. Eur Thyroid J 1:51-54.

540. Appelhof BC, Peeters RP, Wiersinga WM, Visser TJ, Wekking EM, Huyser J, Schene AH, Tijssen JG, Hoogendijk 
WJ, Fliers E 2005 Polymorphisms in type 2 deiodinase are not associated with well-being, neurocognitive functioning, and preference for combined thyroxine/3,5,3'-triiodothyronine therapy. J Clin Endocrinol Metab 90:6296-6299.

541. van der Deure WM, Appelhof BC, Peeters RP, Wiersinga WM, Wekking EM, Huyser J, Schene AH, Tijssen JG, Hoogendijk WJ, Visser TJ, Fliers E 2008 Polymorphisms in the brain-specific thyroid hormone transporter OATP1C1 are associated with fatigue and depression in hypothyroid patients. Clin Endocrinol 69:804-811.

542. Kakucska I, Rand W, Lechan RM 1992 Thyrotropinreleasing hormone gene expression in the hypothalamic paraventricular nucleus is dependent upon feedback regulation by both triiodothyronine and thyroxine. Endocrinology 130:2845-2850.

543. Nicoloff JT, Low JC, Dussault JH, Fisher DA 1972 Simultaneous measurement of thyroxine and triiodothyronine peripheral turnover kinetics in man. J Clin Invest 51: 473-483.

544. Cooper DS, Doherty GM, Haugen BR, Kloos RT, Lee SL, Mandel SJ, Mazzaferri EL, McIver B, Pacini F, Schlumberger M, Sherman SI, Steward DL, Tuttle RM 2009 Revised American Thyroid Association management guidelines for patients with thyroid nodules and differentiated thyroid cancer. Thyroid 19:1167-1214.

545. Hennemann G, Docter R, Visser TJ, Postema PT, Krenning EP 2004 Thyroxine plus low-dose, slow-release triiodothyronine replacement in hypothyroidism: proof of principle. Thyroid 14:271-275.

546. Santini F, Giannetti M, Ricco I, Querci G, Saponati G, Bokor D, Rivolta G, Bussi S, Braverman LE, Vitti P, Pinchera A 2014 Steady state serum T3 concentrations for 48 hours following the oral administration of a single dose of 3,5,3'-triiodothyronine sulfate (T3S). Endocr Pract 20: 680-689.

547. Moeller LC, Broecker-Preuss M 2011 Transcriptional regulation by nonclassical action of thyroid hormone. Thyroid Res 4(Suppl 1):S6.

548. Larsen PR 1972 Triiodothyronine: review of recent studies of its physiology and pathophysiology in man. Metabolism 21:1073-1092.

549. Walfish PG 1976 Triiodothyronine and thyroxine interrelationships in health and disease. Can Med Assoc J 115:338-342.

550. Danzi S, Klein I 2005 Potential uses of T3 in the treatment of human disease. Clin Cornerstone 7(Suppl 2):S9-S15.

551. Cooper-Kazaz R, Lerer B 2008 Efficacy and safety of triiodothyronine supplementation in patients with major depressive disorder treated with specific serotonin reuptake inhibitors. Int J Neuropsychopharmacol 11:685-699.

552. Kirkegaard C, Faber J 1998 The role of thyroid hormones in depression. Eur J Endocrinol 138:1-9.

553. Heal DJ, Smith SL 1988 The effects of acute and repeated administration of $\mathrm{T}_{3}$ to mice on 5-HT1 and 5-HT2 function in the brain and its influence on the actions of repeated electroconvulsive shock. Neuropharmacology 27:12391248.

554. Aronson R, Offman HJ, Joffe RT, Naylor CD 1996 Triiodothyronine augmentation in the treatment of refractory depression. A meta-analysis. Arch Gen Psychiatry 53:842-848.

555. Altshuler LL, Bauer M, Frye MA, Gitlin MJ, Mintz J, Szuba MP, Leight KL, Whybrow PC 2001 Does thyroid supplementation accelerate tricyclic antidepressant response? A review and meta-analysis of the literature. Am J Psychiatry 158:1617-1622.

556. Nierenberg AA, Fava M, Trivedi MH, Wisniewski SR, Thase ME, McGrath PJ, Alpert JE, Warden D, Luther JF, Niederehe G, Lebowitz B, Shores-Wilson K, Rush AJ 2006 A comparison of lithium and T(3) augmentation following two failed medication treatments for depression: a STAR*D report. Am J Psychiatry 163:1519-1530; quiz 1665.

557. American Psychiatric Association 2010 Practice Guideline for the Treatment of Patients With Major Depressive Disorder. Third edition. Available at: http://psychiatryonline .org/content.aspx ?bookid $=28 \&$ sectionid $=1667485$.

558. Moreira-Andres MN, Del Canizo-Gomez FJ, Black EG, Hoffenberg R 1981 Long-term evaluation of thyroidal response to partial calorie restriction in obesity. Clin Endocrinol 15:621-626.

559. U.S. Food and Drug Administration 2007 Pharmacy compounding/compounding of bio-identical hormone replacement therapies. Available at: http://www.fda.gov/ NewsEvents/Testimony/ucm154031.htm.

560. U.S. Food and Drug Administration 2014 The special risks of pharmacy compounding. Available at: http://www.fda .gov/forconsumers/consumerupdates/ucm107836.htm.

561. Snyder S, Listecki RE 2012 Bioidentical thyroid replacement therapy in practice: Delivering a physiologic T4:T3 ratio for improved patient outcomes with the Listecki-Snyder protocol. Int J Pharm Compd 16:376-380.

562. Boulton DW, Fawcett JP, Woods DJ 1996 Stability of an extemporaneously compounded levothyroxine sodium oral liquid. Am J Health Syst Pharm 53:1157-1161.

563. Jha S, Waghdhare S, Reddi R, Bhattacharya P 2012 Thyroid storm due to inappropriate administration of a compounded thyroid hormone preparation successfully treated with plasmapheresis. Thyroid 22:1283-1286.

564. Zeisel SH 1999 Regulation of "nutraceuticals". Science 285:1853-1855.

565. Rosen JE, Gardiner P, Lee SL 2013 Complementary and integrative treatments: thyroid disease. Otolaryngol Clin North Am 46:423-435.

566. Rosen JE, Gardiner P, Saper RB, Filippelli AC, White LF, Pearce EN, Gupta-Lawrence RL, Lee SL 2013 Complementary and alternative medicine use among patients with thyroid cancer. Thyroid 23:1238-1246.

567. Mechanick JI, Brett EM, Chausmer AB, Dickey RA, Wallach S 2003 American Association of Clinical Endocrinologists medical guidelines for the clinical use of dietary supplements and nutraceuticals. Endocr Pract 9:417-470.

568. Hoang TD, Mai VQ, Clyde PW, Shakir MK 2013 Overthe-counter-drug-induced thyroid disorders. Endocr Pract 19:268-274.

569. Boehm TM, Burman KD, Barnes S, Wartofsky L 1980 Lithium and iodine combination therapy for thyrotoxicosis. Acta Endocrinol (Copenh) 94:174-183.

570. Wolff J, Chaikoff IL 1948 Plasma inorganic iodide as a homeostatic regulator of thyroid function. J Biol Chem 174:555-564.

571. Kang GY, Parks JR, Fileta B, Chang A, Abdel-Rahim MM, Burch HB, Bernet VJ 2013 Thyroxine and triiodothyronine content in commercially available thyroid health supplements. Thyroid 23:1233-1237.

572. Sathyapalan T, Manuchehri AM, Thatcher NJ, Rigby AS, Chapman T, Kilpatrick ES, Atkin SL 2011 The effect of soy phytoestrogen supplementation on thyroid status and 
cardiovascular risk markers in patients with subclinical hypothyroidism: a randomized, double-blind, crossover study. J Clin Endocrinol Metab 96:1442-1449.

573. Beck-Peccoz P, Piscitelli G, Cattaneo MG, Faglia G 1983 Successful treatment of hyperthyroidism due to nonneoplastic pituitary TSH hypersecretion with 3,5,3'-triiodothyroacetic acid (TRIAC). J Endocrinol Invest 6:217-223.

574. Bauer BA, Elkin PL, Erickson D, Klee GG, Brennan MD 2002 Symptomatic hyperthyroidism in a patient taking the dietary supplement tiratricol. Mayo Clin Proc 77:587-590.

575. Scally MC, Hodge A 2003 A report of hypothyroidism induced by an over-the-counter fat loss supplement (Tiratricol). Int J Sport Nutr Exerc Metab 13:112-116.

576. Chan WB, Chow CC, Cockram CS 2004 A patient with low free T4 and low thyroid-stimulating hormone without hypopituitarism. Int J Clin Pract 58:983-984.

577. Ma RC, Chan MH, Poon WT, So WY, Chow CC 2008 Thyroid dysfunction due to over-the-counter usage of tiratricol. Intern Med J 38:611-612.

578. Cohen-Lehman J, Charitou MM, Klein I 2011 Tiratricolinduced periodic paralysis: a review of nutraceuticals affecting thyroid function. Endocr Pract 17:610-615.

579. Moreno M, de Lange P, Lombardi A, Silvestri E, Lanni A, Goglia F 2008 Metabolic effects of thyroid hormone derivatives. Thyroid 18:239-253.

580. Antonelli A, Fallahi P, Ferrari SM, Di Domenicantonio A, Moreno M, Lanni A, Goglia F 2011 3,5-diiodo-L-thyronine increases resting metabolic rate and reduces body weight without undesirable side effects. J Biol Regul Homeost Agents 25:655-660.

581. International Committee on Medical Journal Editors 2013 Uniform requirements for manuscripts submitted to biomedical journals. Available at: http://www.icmje.org/ icmje-recommendations.pdf.

582. Freedman B 1987 Equipoise and the ethics of clinical research. N Engl J Med 317:141-145.

583. Barnes B 1976 Hypothyroidism: The Unsuspected Illness. Harper Collins, New York, NY.

584. Blanchard KR 2004 Dosage recommendations for combination regimen of thyroxine and 3,5,3'-triiodothyronine. J Clin Endocrinol Metab 89:1486-1487; author reply 1487-1488.

585. Gabler NB, Duan N, Vohra S, Kravitz RL 2011 N-of-1 trials in the medical literature: a systematic review. Med Care 49:761-768.

586. Friedman M, Miranda-Massari JR, Gonzalez MJ 2006 Supraphysiological cyclic dosing of sustained release $T_{3}$ in order to reset low basal body temperature. P R Health Sci J 25:23-29.

587. WilsonsTemperatureSyndrome.com 1991-2014 Wilson's syndrome doctor's manual. Available at: http://www.wilsons syndrome.com/eManual/.

588. Wilson D 1991 Wilson's temperature syndrome-a reversible low temperature problem. WilsonsTemperatureSyndrome.com, Lady Lake, FL.

589. American Thyroid Association Statement on "Wilson's Syndrome." Statement Updated May 24, 2005. Available at: www.thyroid.org/american-thyroid-association-statementon-wilsons-syndrome/

590. Wikipedia. Wilson's temperature syndrome. Available at: https://en.wikipedia.org/wiki/Wilson\%27s_temperature_ syndrome.

591. Tittle CR 1956 Effects of 3,5,3' L-triiodothyronine in patients with metabolic insufficiency; preliminary report. J Am Med Assoc 162:271-274.
592. Borst GC, Osburne RC, O’Brian JT, Georges LP, Burman KD 1983 Fasting decreases thyrotropin responsiveness to thyrotropin-releasing hormone: a potential cause of misinterpretation of thyroid function tests in the critically ill. J Clin Endocrinol Metab 57:380-383.

593. Lee WY, Kang MI, Oh KW, Oh ES, Baek KH, Lee KW, Kim SW, Kim DW, Min WS, Kim CC 2004 Relationship between circulating cytokine levels and thyroid function following bone marrow transplantation. Bone Marrow Transplant 33:93-98.

594. Imberti R, Ferrari M, Albertini R, Rizzo V, Tinelli C 2010 Increased levothyroxine requirements in critically ill patients with hypothyroidism. Minerva Anestesiol 76:500-503.

595. Yamamoto T, Fukuyama J, Fujiyoshi A 1999 Factors associated with mortality of myxedema coma: report of eight cases and literature survey. Thyroid 9:1167-1174.

596. Peterson RE, Wyngaarden JB, Guerra SL, Brodie BB, Bunim JJ 1955 The physiological disposition and metabolic fate of hydrocortisone in man. J Clin Invest 34: 1779-1794.

597. Paull AM, Phillips RW 1954 Primary myxedema with secondary adrenocortical failure. J Clin Endocrinol Metab 14:554-560.

598. Peterson RE 1958 The influence of the thyroid on adrenal cortical function. J Clin Invest 37:736-743.

599. Rodriguez I, Fluiters E, Perez-Mendez LF, Luna R, Paramo C, Garcia-Mayor RV 2004 Factors associated with mortality of patients with myxoedema coma: prospective study in 11 cases treated in a single institution. $\mathrm{J}$ Endocrinol 180:347-350.

600. Summers VK 1953 Myxoedema coma. Br Med J 2:366368.

601. Le Marquand HS, Hausmann W, Hemsted EH 1953 Myxoedema as a cause of death; report of two cases. $\mathrm{Br}$ Med J 1:704-706.

602. Report of a committee of the Clinical Society of London to investigate the subject of myxedema. Trans Clin Soc (Lond) (suppl) 1888:21.

603. Weinberg AD, Brennan MD, Gorman CA, Marsh HM, O'Fallon WM 1983 Outcome of anesthesia and surgery in hypothyroid patients. Arch Intern Med 143:893-897.

604. Ladenson PW, Goldenheim PD, Cooper DS, Miller MA, Ridgway EC 1982 Early peripheral responses to intravenous L-thyroxine in primary hypothyroidism. Am J Med 73:467-474.

605. Ladenson PW, Goldenheim PD, Ridgway EC 1983 Rapid pituitary and peripheral tissue responses to intravenous Ltriiodothyronine in hypothyroidism. J Clin Endocrinol Metab 56:1252-1259.

606. Dutta P, Bhansali A, Masoodi SR, Bhadada S, Sharma N, Rajput R 2008 Predictors of outcome in myxoedema coma: a study from a tertiary care centre. Crit Care 12:R1.

607. Fliers E, Wiersinga WM 2003 Myxedema coma. Rev Endocr Metab Disord 4:137-141.

608. Goldberg PA, Inzucchi SE 2003 Critical issues in endocrinology. Clin Chest Med 24:583-606, vi.

609. Kaptein EM, Quion-Verde H, Swinney RS, Egodage PM, Massry SG 1986 Acute hemodynamic effects of levothyroxine loading in critically ill hypothyroid patients. Arch Intern Med 146:662-666.

610. Klubo-Gwiezdzinska J, Wartofsky L 2012 Thyroid emergencies. Med Clin North Am 96:385-403.

611. Pereira VG, Haron ES, Lima-Neto N, Medeiros-Neto GA 1982 Management of myxedema coma: report on three 
successfully treated cases with nasogastric or intravenous administration of triiodothyronine. J Endocrinol Invest 5:331-334.

612. Smallridge RC 1992 Metabolic and anatomic thyroid emergencies: a review. Crit Care Med 20:276-291.

613. Wartofsky L 2006 Myxedema coma. Endocrinol Metab Clin North Am 35:687-698, vii-viii.

614. Hylander B, Rosenqvist U 1985 Treatment of myxoedema coma-factors associated with fatal outcome. Acta Endocrinol (Copenh) 108:65-71.

615. Holvey DN, Goodner CJ, Nicoloff JT, Dowling JT 1964 Treatment of myxedema coma with intravenous thyroxine. Arch Intern Med 113:89-96.

616. Arlot S, Debussche X, Lalau JD, Mesmacque A, Tolani M, Quichaud J, Fournier A 1991 Myxoedema coma: response of thyroid hormones with oral and intravenous high-dose L-thyroxine treatment. Intens Care Med 17: 16-18.

617. Beynon J, Akhtar S, Kearney T 2008 Predictors of outcome in myxoedema coma. Crit Care 12:111.

618. MacKerrow SD, Osborn LA, Levy H, Eaton RP, Economou P 1992 Myxedema-associated cardiogenic shock treated with intravenous triiodothyronine. Ann Intern Med 117:1014-1015.

619. Mathew V, Misgar RA, Ghosh S, Mukhopadhyay P, Roychowdhury P, Pandit K, Mukhopadhyay S, Chowdhury S 2011 Myxedema coma: a new look into an old crisis. J Thyroid Res 2011:493462.

620. Jo S, Kallo I, Bardoczi Z, Arrojo e Drigo R, Zeold A, Liposits Z, Oliva A, Lemmon VP, Bixby JL, Gereben B, Bianco AC 2012 Neuronal hypoxia induces Hsp40mediated nuclear import of type 3 deiodinase as an adaptive mechanism to reduce cellular metabolism. J Neurosci 32:8491-8500.

621. Freitas BC, Gereben B, Castillo M, Kallo I, Zeold A, Egri P, Liposits Z, Zavacki AM, Maciel RM, Jo S, Singru P, Sanchez E, Lechan RM, Bianco AC 2010 Paracrine signaling by glial cell-derived triiodothyronine activates neuronal gene expression in the rodent brain and human cells. J Clin Invest 120:2206-2217.

622. Van den Berghe G, de Zegher F, Baxter RC, Veldhuis JD, Wouters P, Schetz M, Verwaest C, Van der Vorst E, Lauwers P, Bouillon R, Bowers CY 1998 Neuroendocrinology of prolonged critical illness: effects of exogenous thyrotropin-releasing hormone and its combination with growth hormone secretagogues. J Clin Endocrinol Metab 83:309-319.

623. Docter R, Krenning EP, de Jong M, Hennemann G 1993 The sick euthyroid syndrome: changes in thyroid hormone serum parameters and hormone metabolism. Clin Endocrinol 39:499-518.

624. De Groot LJ 2006 Non-thyroidal illness syndrome is a manifestation of hypothalamic-pituitary dysfunction, and in view of current evidence, should be treated with appropriate replacement therapies. Crit Care Clin 22:57-86, vi.

625. Lechan RM 2008 The dilemma of the nonthyroidal illness syndrome. Acta Biomed 79:165-171.

626. Hollenberg AN 2008 The role of the thyrotropin-releasing hormone (TRH) neuron as a metabolic sensor. Thyroid 18:131-139.

627. Mohacsik P, Zeold A, Bianco AC, Gereben B 2011 Thyroid hormone and the neuroglia: both source and target. J Thyroid Res 2011:215718.
628. Boelen A, Kwakkel J, Fliers E 2011 Beyond low plasma $\mathrm{T}_{3}$ : local thyroid hormone metabolism during inflammation and infection. Endocr Rev 32:670-693.

629. Acker CG, Singh AR, Flick RP, Bernardini J, Greenberg A, Johnson JP 2000 A trial of thyroxine in acute renal failure. Kidney Int 57:293-298.

630. Van den Berghe G, de Zegher F, Lauwers P 1994 Dopamine and the sick euthyroid syndrome in critical illness. Clin Endocrinol 41:731-737.

631. Pappa TA, Vagenakis AG, Alevizaki M 2011 The nonthyroidal illness syndrome in the non-critically ill patient. Eur J Clin Invest 41:212-220.

632. Kaptein EM, Sanchez A, Beale E, Chan LS 2010 Clinical review: thyroid hormone therapy for postoperative nonthyroidal illnesses: a systematic review and synthesis. J Clin Endocrinol Metab 95:4526-4534.

633. Marks SD 2009 Nonthyroidal illness syndrome in children. Endocrine 36:355-367.

634. Peeters RP 2007 Non thyroidal illness: to treat or not to treat? Ann Endocrinol (Paris) 68:224-228.

635. McIver B, Gorman CA 1997 Euthyroid sick syndrome: an overview. Thyroid 7:125-132.

636. Chopra IJ 1997 Clinical review 86: Euthyroid sick syndrome: is it a misnomer? J Clin Endocrinol Metab 82:329-334.

637. Brent GA, Hershman JM 1986 Thyroxine therapy in patients with severe nonthyroidal illnesses and low serum thyroxine concentration. J Clin Endocrinol Metab 63:1-8.

638. Becker RA, Vaughan GM, Ziegler MG, Seraile LG, Goldfarb IW, Mansour EH, McManus WF, Pruitt BA Jr, Mason AD Jr 1982 Hypermetabolic low triiodothyronine syndrome of burn injury. Crit Care Med 10:870-875.

639. Moruzzi P, Doria E, Agostoni PG 1996 Medium-term effectiveness of L-thyroxine treatment in idiopathic dilated cardiomyopathy. Am J Med 101:461-467.

640. Moruzzi P, Doria E, Agostoni PG, Capacchione V, Sganzerla P 1994 Usefulness of L-thyroxine to improve cardiac and exercise performance in idiopathic dilated cardiomyopathy. Am J Cardiol 73:374-378.

641. Lu X, Huang J, Zhang X, Li X, Wang C, Zhang P, Chen Y 2003 Effects of thyroxine on cardiac function and lymphocyte beta-adrenoceptors in patients with chronic congestive heart failure. Chin Med J (Engl) 116:1697-1700.

642. Sirlak M, Yazicioglu L, Inan MB, Eryilmaz S, Tasoz R, Aral A, Ozyurda U 2004 Oral thyroid hormone pretreatment in left ventricular dysfunction. Eur J Cardiothorac Surg 26:720-725.

643. Pingitore A, Galli E, Barison A, Iervasi A, Scarlattini M, Nucci D, L'Abbate A, Mariotti R, Iervasi G 2008 Acute effects of triiodothyronine (T3) replacement therapy in patients with chronic heart failure and low-T3 syndrome: a randomized, placebo-controlled study. J Clin Endocrinol Metab 93:1351-1358.

644. Acker CG, Flick R, Shapiro R, Scantlebury VP, Jordan ML, Vivas C, Greenberg A, Johnson JP 2002 Thyroid hormone in the treatment of post-transplant acute tubular necrosis (ATN). Am J Transplant 2:57-61.

645. Peeters RP, van der Geyten S, Wouters PJ, Darras VM, van Toor H, Kaptein E, Visser TJ, Van den Berghe G 2005 Tissue thyroid hormone levels in critical illness. J Clin Endocrinol Metab 90:6498-6507.

646. Galli E, Pingitore A, Iervasi G 2010 The role of thyroid hormone in the pathophysiology of heart failure: clinical evidence. Heart failure reviews 15:155-169. 
647. Danzi S, Klein I 2012 Thyroid hormone and the cardiovascular system. Med Clin North Am 96:257-268.

648. Hamilton MA, Stevenson LW, Fonarow GC, Steimle A, Goldhaber JI, Child JS, Chopra IJ, Moriguchi JD, Hage A 1998 Safety and hemodynamic effects of intravenous triiodothyronine in advanced congestive heart failure. Am J Cardiol 81:443-447.

649. Pingitore A, Iervasi G 2008 Triiodothyronine (T3) effects on cardiovascular system in patients with heart failure. Recent Pat Cardiovasc Drug Discov 3:19-27.

650. D’Aloia A, Vizzardi E, Bugatti S, Rovetta R, Bonadei I, Del Magro F, Curnis A, Dei Cas L 2012 Effect of shortterm infusive dobutamine therapy on thyroid hormone profile and hemodynamic parameters in patients with acute worsening heart failure and low-triiodothyronine syndrome. J Investig Med 60:907-910.

651. Shiohara H, Nakamura T, Kikuchi N, Ozawa T, Nagano R, Matsuzawa A, Ohnota H, Miyamoto T, Ichikawa K, Hashizume K 2012 Discovery of novel indane derivatives as liver-selective thyroid hormone receptor beta (TRbeta) agonists for the treatment of dyslipidemia. Bioorg Med Chem 20:3622-3634.

652. Chiellini G, Apriletti JW, Yoshihara HA, Baxter JD, Ribeiro RC, Scanlan TS 1998 A high-affinity subtypeselective agonist ligand for the thyroid hormone receptor. Chem Biol 5:299-306.

653. Ribeiro MO 2008 Effects of thyroid hormone analogs on lipid metabolism and thermogenesis. Thyroid 18:197203.

654. Erion MD, Reddy KR, Boyer SH, Matelich MC, GomezGaleno J, Lemus RH, Ugarkar BG, Colby TJ, Schanzer J, Van Poelje PD 2004 Design, synthesis, and characterization of a series of cytochrome $\mathrm{P}(450) 3 \mathrm{~A}$-activated prodrugs (HepDirect prodrugs) useful for targeting phosph(on)ate-based drugs to the liver. J Am Chem Soc 126: 5154-5163.

655. Martinez L, Nascimento AS, Nunes FM, Phillips K, Aparicio R, Dias SM, Figueira AC, Lin JH, Nguyen P, Apriletti JW, Neves FA, Baxter JD, Webb P, Skaf MS, Polikarpov I 2009 Gaining ligand selectivity in thyroid hormone receptors via entropy. Proc Natl Acad Sci U S A 106:20717-20722.

656. Meruvu S, Ayers SD, Winnier G, Webb P 2013 Thyroid hormone analogues: where do we stand in 2013? Thyroid 23:1333-1344.

657. Ito BR, Zhang BH, Cable EE, Song X, Fujitaki JM, MacKenna DA, Wilker CE, Chi B, van Poelje PD, Linemeyer DL, Erion MD 2009 Thyroid hormone beta receptor activation has additive cholesterol lowering activity in combination with atorvastatin in rabbits, dogs and monkeys. Br J Pharmacol 156:454-465.

658. Trost SU, Swanson E, Gloss B, Wang-Iverson DB, Zhang H, Volodarsky T, Grover GJ, Baxter JD, Chiellini G, Scanlan TS, Dillmann WH 2000 The thyroid hormone receptor-beta-selective agonist GC-1 differentially affects plasma lipids and cardiac activity. Endocrinology 141: 3057-3064.

659. Freitas FR, Moriscot AS, Jorgetti V, Soares AG, Passarelli M, Scanlan TS, Brent GA, Bianco AC, Gouveia CH 2003 Spared bone mass in rats treated with thyroid hormone receptor TR beta-selective compound GC-1. Am J Physiol Endocrinol Metab 285:E1135-1141.

660. Amorim BS, Ueta CB, Freitas BC, Nassif RJ, Gouveia $\mathrm{CH}$, Christoffolete MA, Moriscot AS, Lancelloti CL,
Llimona F, Barbeiro HV, de Souza HP, Catanozi S, Passarelli M, Aoki MS, Bianco AC, Ribeiro MO 2009 A TRbeta-selective agonist confers resistance to diet-induced obesity. J Endocrinol 203:291-299.

661. Grover GJ, Egan DM, Sleph PG, Beehler BC, Chiellini G, Nguyen NH, Baxter JD, Scanlan TS 2004 Effects of the thyroid hormone receptor agonist GC-1 on metabolic rate and cholesterol in rats and primates: selective actions relative to 3,5,3'-triiodo-L-thyronine. Endocrinology 145: 1656-1661.

662. Grover GJ, Mellstrom K, Malm J 2005 Development of the thyroid hormone receptor beta-subtype agonist KB141: a strategy for body weight reduction and lipid lowering with minimal cardiac side effects. Cardiovasc Drug Rev 23:133-148.

663. Anzai R, Adachi M, Sho N, Muroya K, Asakura Y, Onigata K 2012 Long-term 3,5,3'-triiodothyroacetic acid therapy in a child with hyperthyroidism caused by thyroid hormone resistance: pharmacological study and therapeutic recommendations. Thyroid 22:1069-1075.

664. Pennock GD, Raya TE, Bahl JJ, Goldman S, Morkin E 1992 Cardiac effects of 3,5-diiodothyropropionic acid, a thyroid hormone analog with inotropic selectivity. J Pharmacol Exp Ther 263:163-169.

665. Pennock GD, Raya TE, Bahl JJ, Goldman S, Morkin E 1993 Combination treatment with captopril and the thyroid hormone analogue 3,5-diiodothyropropionic acid. A new approach to improving left ventricular performance in heart failure. Circulation 88:1289-1298.

666. Mahaffey KW, Raya TE, Pennock GD, Morkin E, Goldman S 1995 Left ventricular performance and remodeling in rabbits after myocardial infarction. Effects of a thyroid hormone analogue. Circulation 91:794-801.

667. Talukder MA, Yang F, Nishijima Y, Chen CA, Xie L, Mahamud SD, Kalyanasundaram A, Bonagura JD, Periasamy M, Zweier JL 2011 Detrimental effects of thyroid hormone analog DITPA in the mouse heart: increased mortality with in vivo acute myocardial ischemiareperfusion. Am J Physiol Heart Circ Physiol 300:H702711.

668. Ladenson PW, Kristensen JD, Ridgway EC, Olsson AG, Carlsson B, Klein I, Baxter JD, Angelin B 2010 Use of the thyroid hormone analogue eprotirome in statin-treated dyslipidemia. N Engl J Med 362:906-916.

669. Berkenstam A, Kristensen J, Mellstrom K, Carlsson B, Malm J, Rehnmark S, Garg N, Andersson CM, Rudling M, Sjoberg F, Angelin B, Baxter JD 2008 The thyroid hormone mimetic compound KB2115 lowers plasma LDL cholesterol and stimulates bile acid synthesis without cardiac effects in humans. Proc Natl Acad Sci U S A 105:663-667.

670. Angelin B, Kristensen JD, Eriksson M, Carlsson B, Klein I, Olsson AG, Chester Ridgway E, Ladenson PW 2014 Reductions in serum levels of LDL cholesterol, apolipoprotein B, triglycerides and lipoprotein(a) in hypercholesterolaemic patients treated with the liver-selective thyroid hormone receptor agonist eprotirome. J Intern Med [Epub ahead of print]; DOI: 10.1111/joim.12261.

671. Sjouke B, Langslet G, Ceska R, Nicholls SJ, Nissen SE, Ohlander M, Ladenson PW, Olsson AG, Hovingh GK, Kastelein JJP 2014 Eprotirome in patients with familial hypercholesterolaemia (the AKKA trial): a randomised, double-blind, placebo-controlled phase 3 study. Lancet Diabetes Endocrinol 2:455-463. 
672. Karo Bio AB 2012 Karo Bio terminates the eprotirome program. Available at: http://www.karobio.com/investor media/pressreleaser/pressrelease pid $=639535$.

673. Goldman S, McCarren M, Morkin E, Ladenson PW, Edson R, Warren S, Ohm J, Thai H, Churby L, Barnhill J, O'Brien T, Anand I, Warner A, Hattler B, Dunlap M, Erikson J, Shih MC, Lavori P 2009 DITPA (3,5-diiodothyropropionic acid), a thyroid hormone analog to treat heart failure: phase II trial veterans affairs cooperative study. Circulation 119:3093-3100.

674. Ladenson PW, McCarren M, Morkin E, Edson RG, Shih MC, Warren SR, Barnhill JG, Churby L, Thai H, O’Brien T, Anand I, Warner A, Hattler B, Dunlap M, Erikson J, Goldman S 2010 Effects of the thyromimetic agent diiodothyropropionic acid on body weight, body mass index, and serum lipoproteins: a pilot prospective, randomized, controlled study. J Clin Endocrinol Metab 95: 1349-1354.

675. Sherman SI, Ladenson PW 1992 Organ-specific effects of tiratricol: a thyroid hormone analog with hepatic, not pituitary, superagonist effects. J Clin Endocrinol Metab 75:901-905.

676. Sherman SI, Ringel MD, Smith MJ, Kopelen HA, Zoghbi WA, Ladenson PW 1997 Augmented hepatic and skeletal thyromimetic effects of tiratricol in comparison with levothyroxine. J Clin Endocrinol Metab 82:2153-2158.

677. Refetoff S, DeWind LT, DeGroot LJ 1967 Familial syndrome combining deaf-mutism, stuppled epiphyses, goiter and abnormally high PBI: possible target organ refractoriness to thyroid hormone. J Clin Endocrinol Metab 27: 279-294.

678. Gershengorn MC, Weintraub BD 1975 Thyrotropininduced hyperthyroidism caused by selective pituitary resistance to thyroid hormone. A new syndrome of "inappropriate secretion of TSH". J Clin Invest 56:633-642.

679. Sakurai A, Takeda K, Ain K, Ceccarelli P, Nakai A, Seino S, Bell GI, Refetoff S, DeGroot LJ 1989 Generalized resistance to thyroid hormone associated with a mutation in the ligand-binding domain of the human thyroid hormone receptor beta. Proc Natl Acad Sci U S A 86:8977-8981.

680. Parikh S, Ando S, Schneider A, Skarulis MC, Sarlis NJ, Yen PM 2002 Resistance to thyroid hormone in a patient without thyroid hormone receptor mutations. Thyroid 12:81-86.

681. Weiss RE, Marcocci C, Bruno-Bossio G, Refetoff S 1993 Multiple genetic factors in the heterogeneity of thyroid hormone resistance. J Clin Endocrinol Metab 76:257259.

682. Machado DS, Sabet A, Santiago LA, Sidhaye AR, Chiamolera MI, Ortiga-Carvalho TM, Wondisford FE 2009 A thyroid hormone receptor mutation that dissociates thyroid hormone regulation of gene expression in vivo. Proc Natl Acad Sci U S A 106:9441-9446.

683. Alberobello AT, Congedo V, Liu H, Cochran C, Skarulis MC, Forrest D, Celi FS 2011 An intronic SNP in the thyroid hormone receptor beta gene is associated with pituitary cell-specific over-expression of a mutant thyroid hormone receptor beta2 (R338W) in the index case of pituitary-selective resistance to thyroid hormone. J Transl Med 9:144.

684. Bantle JP, Seeling S, Mariash CN, Ulstrom RA, Oppenheimer JH 1982 Resistance to thyroid hormones. A disorder frequently confused with Graves' disease. Arch Intern Med 142:1867-1871.
685. Beck-Peccoz P, Chatterjee VK 1994 The variable clinical phenotype in thyroid hormone resistance syndrome. Thyroid 4:225-232.

686. Weiss RE, Stein MA, Refetoff S 1997 Behavioral effects of liothyronine (L-T3) in children with attention deficit hyperactivity disorder in the presence and absence of resistance to thyroid hormone. Thyroid 7:389-393.

687. Radetti G, Persani L, Molinaro G, Mannavola D, Cortelazzi D, Chatterjee VK, Beck-Peccoz P 1997 Clinical and hormonal outcome after two years of triiodothyroacetic acid treatment in a child with thyroid hormone resistance. Thyroid 7:775-778.

688. Shi Y, Ye H, Link KH, Putnam MC, Hubner I, Dowdell S, Koh JT 2005 Mutant-selective thyromimetics for the chemical rescue of thyroid hormone receptor mutants associated with resistance to thyroid hormone. Biochemistry 44:4612-4626.

Address correspondence to: Jacqueline Jonklaas, MD, PhD

Division of Endocrinology Georgetown University

Suite 230, Bldg. D, 4000 Reservoir Rd., NW

Washington, DC 20007

E-mail: jonklaaj@georgetown.edu

\begin{tabular}{|c|}
\hline Abbreviations Used \\
\hline $3,5-\mathrm{T}_{2}=3,5$-Diiodothyronine \\
\hline $\begin{array}{c}\mathrm{AACE}=\text { American Association of Clinical } \\
\text { Endocrinologists }\end{array}$ \\
\hline $\mathrm{ACE}=$ Angiotensin-converting enzyme \\
\hline $\mathrm{ACP}=$ American College of Physicians \\
\hline AITD $=$ Autoimmune thyroid disease \\
\hline ATA $=$ American Thyroid Association \\
\hline $\mathrm{AUC}=$ Area under the curve \\
\hline $\mathrm{BMI}=$ Body mass index \\
\hline $\mathrm{CAD}=$ Coronary artery disease \\
\hline $\mathrm{CH}=$ Congenital hypothyroidism \\
\hline $\mathrm{Cmax}=$ Maximum concentration \\
\hline $\mathrm{D} 1=$ Deiodinase type 1 \\
\hline D2 $=$ Deiodinase type 2 \\
\hline D3 $=$ Deiodinase type 3 \\
\hline$D I O 1=$ Deiodinase type 1 gene \\
\hline $\mathrm{DIO} 2=$ Deiodinase type 2 gene \\
\hline$D I O 3=$ Deiodinase type 3 gene \\
\hline DITPA $=3,5$-Diiodothyropropionic acid \\
\hline ETA $=$ European Thyroid Association \\
\hline FDA $=$ U.S. Food and Drug Administration \\
\hline $\mathrm{FT}_{3}=$ Free triiodothyronine \\
\hline $\mathrm{FT}_{4}=$ Free thyroxine \\
\hline $\mathrm{GH}=$ Growth hormone \\
\hline $\mathrm{GHQ}=$ General Health Questionnaire \\
\hline GWAS $=$ Genome-wide association studies \\
\hline $\mathrm{HDL}=$ High-density lipoprotein \\
\hline $\mathrm{ICU}=$ Intensive care unit \\
\hline $\mathrm{IRB}=$ Institutional review board \\
\hline $\begin{array}{c}\mathrm{LC} / \mathrm{MS} / \mathrm{MS}=\begin{array}{c}\text { Liquid chromatography-tandem mass } \\
\text { spectrometry }\end{array}\end{array}$ \\
\hline LDL $=$ Low-density lipoprotein \\
\hline $\mathrm{LT}_{3}=$ Liothyronine \\
\hline
\end{tabular}




\section{Abbreviations Used (Cont.)}

$\mathrm{LT}_{4}=$ Levothyroxine

$\mathrm{MCT}=$ Monocarboxylate transporter

NTIS $=$ Nonthyroidal illness syndrome

OATP $=$ Organic anion transporting polypeptide

$\mathrm{OH}=$ Overt hypothyroidism

PPI $=$ Proton pump inhibitor

PTU $=$ Propylthiouracil

$\mathrm{RCR}=$ Responsible conduct of research

$\mathrm{RCT}=$ Randomized controlled trial

$\mathrm{rT}_{3}=$ Reverse triiodothyronine

$\mathrm{RTH}=$ Resistance to thyroid hormone

$\mathrm{RTH} \alpha=$ Syndrome of resistance to thyroid hormone with mutation in $\mathrm{TR} \alpha$

$\mathrm{RTH} \beta=$ Syndrome of resistance to thyroid hormone with mutation in $\operatorname{TR} \beta$

$\mathrm{SCH}=$ Subclinical hypothyroidism
SHBG $=$ Sex hormone binding globulin

SNPs $=$ Single nucleotide polymorphisms

SSRIs $=$ Serotonin re-uptake inhibitors

$\mathrm{T}_{3}=$ Triiodothyronine

$\mathrm{T}_{4}=$ Thyroxine

$\mathrm{TBG}=$ Thyroxine binding globulin

TCA $=$ Tricyclic antidepressant

$\mathrm{TH}=$ Thyroid hormone

$\mathrm{TPOAb}=$ Thyroid peroxidase antibodies

$\mathrm{TR}=$ Thyroid hormone receptor

TRH $=$ Thyrotropin-releasing hormone

TRIAC $=3,5,3^{\prime}$-triiodothyroacetic acid

$\operatorname{TR} \alpha=$ Thyroid hormone receptor $-\alpha$

$\operatorname{TR} \beta=$ Thyroid hormone receptor- $\beta$

$\mathrm{TSH}=$ Thyrotropin

USP $=$ United States Pharmacopeia 